\title{
Prediction of turbulent mixing at the interface of density stratified, shear flows using CFD
}

\author{
Matthew Robert Umbel \\ West Virginia University
}

Follow this and additional works at: https://researchrepository.wvu.edu/etd

\section{Recommended Citation}

Umbel, Matthew Robert, "Prediction of turbulent mixing at the interface of density stratified, shear flows using CFD" (1998). Graduate Theses, Dissertations, and Problem Reports. 938.

https://researchrepository.wvu.edu/etd/938

This Thesis is protected by copyright and/or related rights. It has been brought to you by the The Research Repository @ WVU with permission from the rights-holder(s). You are free to use this Thesis in any way that is permitted by the copyright and related rights legislation that applies to your use. For other uses you must obtain permission from the rights-holder(s) directly, unless additional rights are indicated by a Creative Commons license in the record and/ or on the work itself. This Thesis has been accepted for inclusion in WVU Graduate Theses, Dissertations, and Problem Reports collection by an authorized administrator of The Research Repository @ WVU. For more information, please contact researchrepository@mail.wvu.edu. 


\section{PREDICTION OF TURBULENT MIXING AT THE INTERFACE OF DENSITY STRATIFIED, SHEAR FLOWS USING CFD}

By

Matthew R. Umbel

\section{A THESIS}

Submitted to

The College of Engineering and Mineral Resources

at

West Virginia University

in partial fulfillment of the requirements

for the degree of

Master of Science

in

Mechanical Engineering

Department of Mechanical and Aerospace Engineering

Morgantown, West Virginia

1998 


\begin{abstract}
Density stratified, shear flows are a common flow phenomena that occur in many engineering applications. Using the commercial code CFX 4.1, several numerical simulations were performed involving various stratified shear flows that have been investigated experimentally. One set of experiments dealt with homogeneous shear, involving fresh water and brine, which are miscible fluids. The other set of experiments dealt with a developing shear layer, involving two immiscible fluids, namely fresh water and diesel fuel. Of primary interest in these simulations was the ability to predict trends for the interfacial thickness and local characteristic Richardson numbers. After these verification/validation studies, the re-fueling of a compensated fuel/ballast tank, which is partially characterized by a shear layer, was also simulated. Compensated fuel/ballast tanks (CFBT) are used in US navy ships and are located in the bottom of the ships. During re-fueling diesel fuel is pumped into a series of tanks through a vertical inlet pipe, forcing the compensating sea water out of the bottom of the tank through an exit pipe, and some of the fuel becomes entrained in the water exiting the tank. Of primary interest in this re-fueling process is the extent to which the fuel and water mix.
\end{abstract}

In all of the simulations, various multiphase models and turbulence models were evaluated. The turbulence models that were used included the standard $k-\varepsilon$ model and a modified form of the $k-\varepsilon$ model that included effects of buoyant production and dissipation of turbulent kinetic energy. The multiphase models that were evaluated included the default CFX homogeneous model and two versions of a single fluid, scalar transport model (SFST), which 
were implemented through user FORTRAN. The SFST models solve one set of momentum equations, but still account for the relative velocity between the phases.

For the simulations involving fresh water and brine, time averaged, steady state solutions were obtained using a single fluid, scalar transport model, and the $k-\varepsilon$ turbulence model. The majority of the experimental parameters were predicted within an acceptable level of error. The maximum velocity gradient thickness, $\mathrm{h}_{\mathrm{S}}$, and the shear Richardson numbers were well predicted in magnitude, although the trends for these parameters that were observed in the experiments were not always seen in the simulations. This was most likely due to the exclusion of low Reynolds number effects, in the $k-\varepsilon$ model that was used in the simulations. The predicted trend for the interfacial thickness, $\delta$, was good for all four cases. An interfacial thickness relationship given by $\delta / \mathrm{H} \sim\left(\mathrm{Ri}^{*}\right)^{-2.1}$ and an entrainment relationship given by $\mathrm{E} \sim$ $(\mathrm{Ri})^{-1.1}$ were predicted by the model and are in agreement with experimental observations.

For the simulations of the mixing layer involving diesel fuel and fresh water, time averaged, steady state solutions were obtained using a single fluid, scalar transport model and the $k-\varepsilon$ turbulence model. Results from these simulations show a strong gradient Richardson number dependence, where the range of gradient Richardson numbers in the simulations ranged from $\mathrm{Ri}_{\mathrm{G}}=0.05$ to $\mathrm{Ri}_{\mathrm{G}}=0.25$. The predicted flow field was confirmed to qualitatively represent the actual flow field that was observed experimentally.

The simulations of the compensated fuel/ballast tank using the SFST model demonstrated the applicability of single fluid scalar transport models to complicated mixing situations where 
relative velocity effects are important. The transient mixing process showed that buoyant flow events around manholes and internal openings are important areas where a significant amount of mixing can occur. The re-fueling efficiency was also shown to be deficient in the geometry that was simulated. 


\section{Acknowledgments}

I would like to acknowledge the support of the persons who provided valuable assistance in the completion of this work. The instructive comments of my committee members, Dr. John Kuhlman and Dr. Rakesh Gupta were helpful in preparing the final draft of the document. The conversations with those at the Naval Surface Warfare Center, namely Peter Chang and Brian Hill, concerning their experience with the fluid simulation code, CFX is also acknowledged and appreciated.

I would like to say thank you for the memories and good luck to my co-workers, whom I aided in throwing a garbage can full of a slimy, orange dough concoction off the engineering building. Also, as the only other CFX user, Wesley Wilson's camaraderie and help with running computer simulations was greatly appreciated.

I would also like to extend special thanks to Dr. Ismail Celik for his assistance during the completion of this project. As my student advisor and supervisor, his insight and direction were invaluable and were greatly appreciated. It has been a tremendous opportunity to work with Dr. Celik on this project involving numerical simulations of multiphase flow and gain both academic and practical engineering experience.

Finally, I would like to extend a special thank-you to my wife, Lauren, for her patience, support, and sympathy during the completion of this thesis. Her assistance in reading the manuscript and inspecting the grammar and punctuation was also appreciated. 


\section{Table of Contents}

\section{Page}

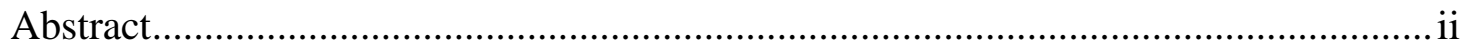

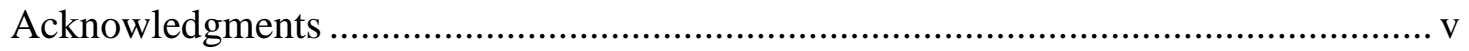

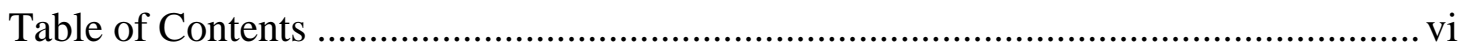

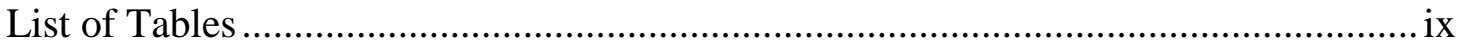

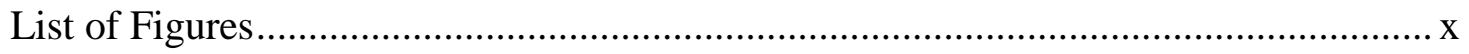

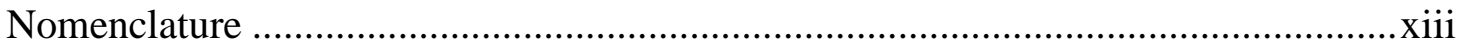

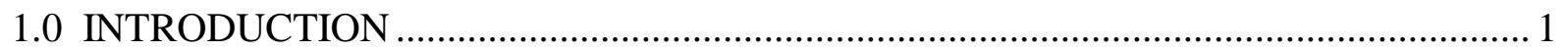

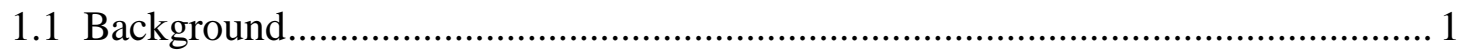

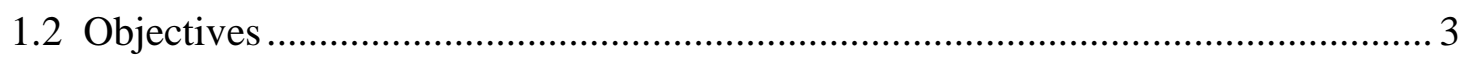

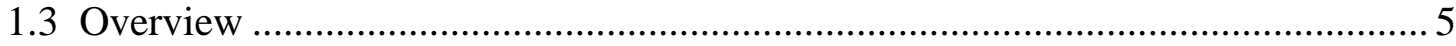

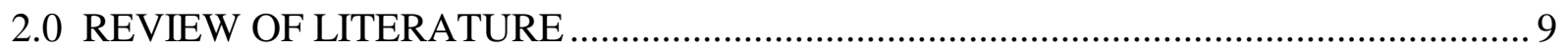

2.1 Introductory Definitions and Concepts...............................................................

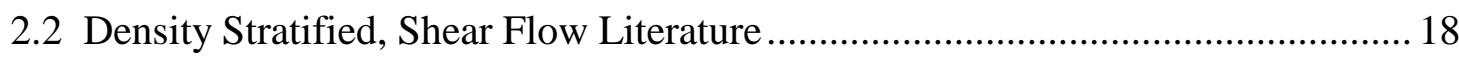

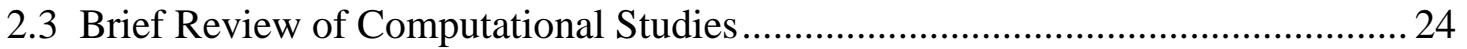

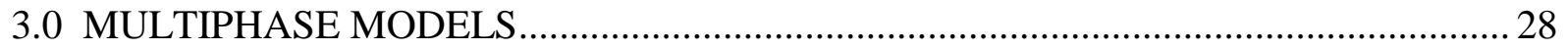

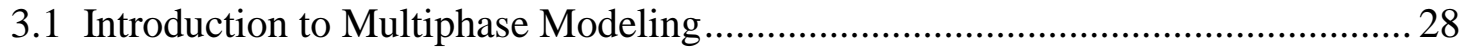

3.2 SFST Model for Flows Involving Immiscible Fluids........................................... 30

3.3 SFST Model for Flows Involving Miscible Fluids............................................. 36 
4.1 Introduction to Turbulence Modeling for Multiphase Flows …………………….... 39

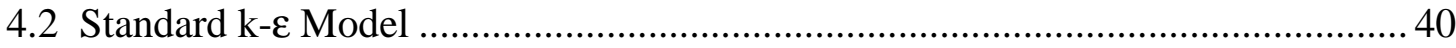

4.3 Modified k- $\varepsilon$ Model for Buoyant Flows ................................................................ 43

5.0 SIMULATION OF STRATIFIED, SHEARING FLOW OF MISCIBLE FLUIDS ...........46

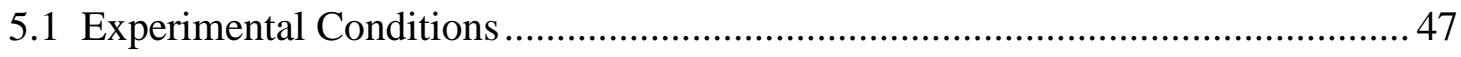

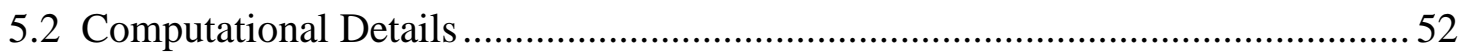

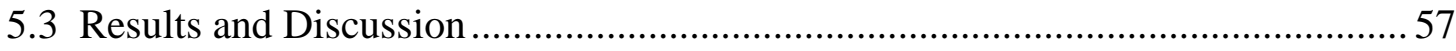

6.0 SIMULATION OF STRATIFIED, SHEARING FLOW OF IMMISCIBLE FLUIDS ...... 80

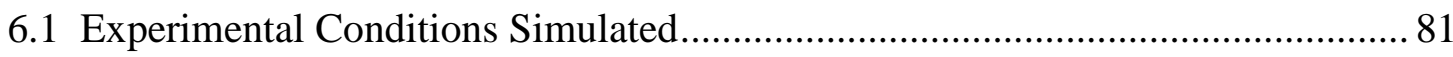

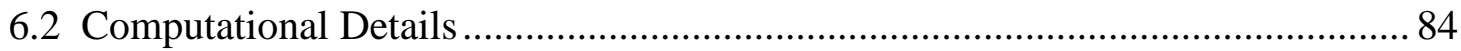

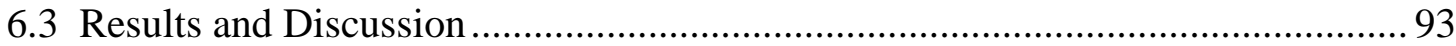

7.0 SIMULATION OF FLOW INSIDE A FULL SCALE CFBT …….................................. 119

7.1 Actual Flow Conditions and Computational Model.............................................. 119

7.2 Computational Details for DDG 51 Tank 5-300-2-F Simulations ........................ 121

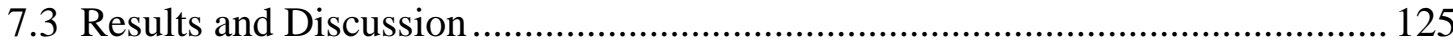

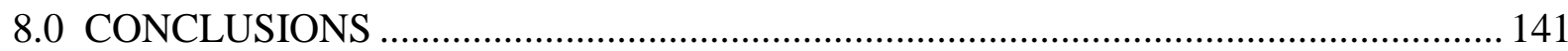

8.1 Summary

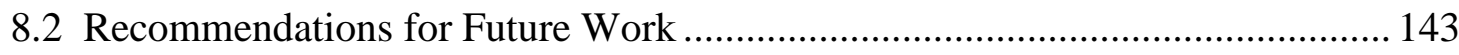

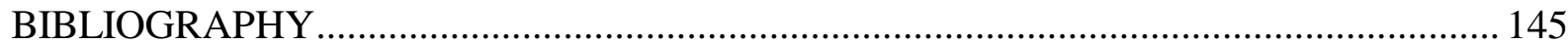


A.1 Model Assumptions, Definitions of Mixture Properties

A.2 Reynolds Averaged Continuity Equation for SFST Model

A.3 Incompressible law for Mixture Velocity ................................................ 151

A.4 Volume Fraction Equation for SFST Model ................................................. 154

A.5 Mixture Momentum Equation for SFST Models ......................................... 156

A.6 Derivation of Terminal Velocity Relationships....................................... 161

APPENDIX B: DOCUMENTATION FOR SFST MODEL ............................................. 164

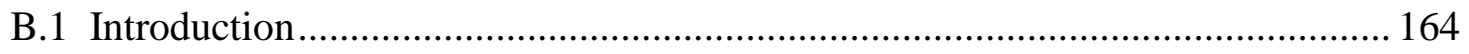

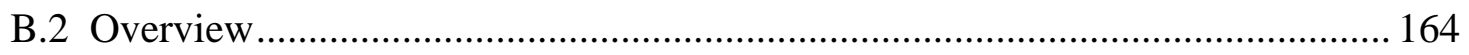

B.3 Setting up the CFX Files and User Fortran to Run the SFST Model ................ 165

B.4 Running a Simulation and Obtaining a Solution ....................................... 171

B.5 Details of Fortran Implementation of SFST Model in CFX ............................ 172 


\section{List of Tables}

Page

Table 5.1 - Experimental flow parameters................................................................52

Table 5.2 - Inlet flow parameters for CFX model......................................................55

Table 5.3 - Computational results and experimental values for Case 1...............................58

Table 5.4 - Computational results and experimental values for Case 10.............................58

Table 5.5 - Computational results and experimental values for Case $11 \ldots \ldots \ldots \ldots \ldots \ldots \ldots \ldots \ldots \ldots \ldots . . .59$

Table 5.6 - Computational results and experimental values for Case 13............................59

Table 6.1 - Parameters used for Johns Hopkins shear flow simulations.............................92 


\section{List of Figures}

Page

Figure 1.1 - Geometry of baseline DDG 51 Tank 5-300-2-F CFBT. ........................................ 1

Figure 2.1 - Idealized density stratified shear flow. .......................................................... 9

Figure 5.1 - Idealized shear flow for Sullivan and List experiments........................................ 46

Figure 5.2 - Sullivan and List experimental setup............................................................ 47

Figure 5.3 - Experimental facility for experiments; After Sullivan [28] ................................ 48

Figure 5.4 - Geometry used in CFX for simulation of Sullivan and List experiments..............53

Figure 5.5 - Position of velocity and concentration interface for case $11 \ldots \ldots \ldots \ldots \ldots \ldots \ldots \ldots \ldots \ldots \ldots . . . .62$

Figure 5.6 - Variation of mixed layer Richardson number with Ri*........................................65

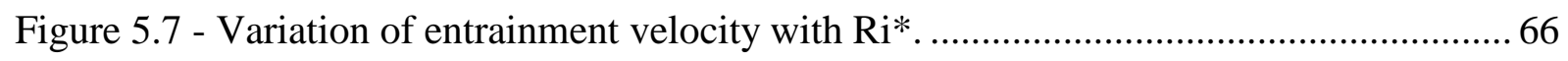

Figure 5.8 - Contours of volume fraction and velocity vectors for case 1 .............................. 67

Figure 5.9 - Contours of volume fraction and velocity vectors for case $10 \ldots \ldots \ldots \ldots \ldots \ldots \ldots \ldots \ldots . . . .67$

Figure 5.10 - Contours of volume fraction and velocity vectors for case $11 \ldots \ldots \ldots \ldots \ldots \ldots \ldots \ldots . . . .68$

Figure 5.11 - Contours of volume fraction and velocity vectors for case 13.........................68

Figure 5.12 - Velocity profile and volume fraction profile for case 1 ....................................... 69

Figure 5.13 - Velocity profile and volume fraction profile for case 10 .................................. 70

Figure 5.14 - Velocity profile and volume fraction profile for case $11 \ldots \ldots \ldots \ldots \ldots \ldots \ldots \ldots \ldots \ldots \ldots \ldots \ldots . . .70$

Figure 5.15 - Velocity profile and volume fraction profile for case $13 \ldots \ldots \ldots \ldots \ldots \ldots \ldots \ldots \ldots \ldots \ldots \ldots \ldots . . .71$

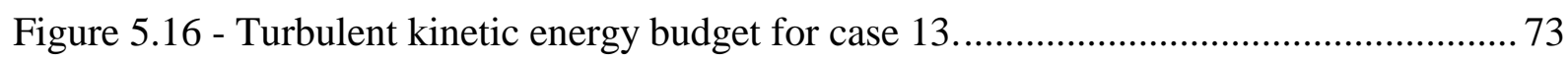

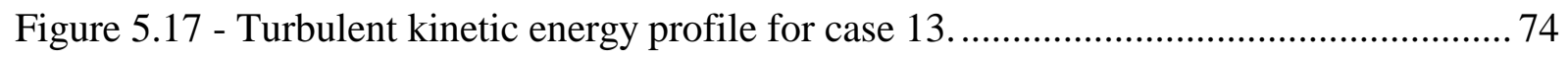

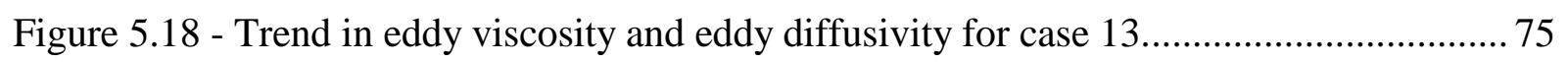


Figure 5.19 - Normalized interfacial thickness versus $\mathrm{Ri}^{*}$. 77

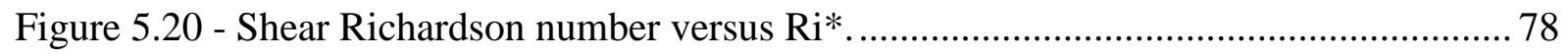

Figure 6.1 - Johns Hopkins shear flow experimental setup.................................................... 81

Figure 6.2 - Johns Hopkins shear flow facility; After Katz 1998 [20].................................... 83

Figure 6.3 - Comparison of drag correlations in Allen's regime............................................ 87

Figure 6.4 - Geometry used in CFX to simulate Johns Hopkins shear flow. ........................... 90

Figure 6.5 - Volume fraction contours and velocity vectors; $\mathrm{Ri}^{*}=0.85, \mathrm{~d}_{\mathrm{p}}=1.75 \mathrm{~mm}$. ........... 94

Figure 6.6 - Streamlines and volume fraction contours; $\mathrm{Ri}^{*}=0.85, \mathrm{~d}_{\mathrm{p}}=1.75 \mathrm{~mm} \ldots \ldots \ldots \ldots \ldots \ldots \ldots . .95$

Figure 6.7 - Volume fraction contours; $\mathrm{Ri}^{*}=0.85, \mathrm{~d}_{\mathrm{p}}=1.75 \mathrm{~mm}$........................................ 96

Figure 6.8 - Streamlines and volume fraction contours; $\mathrm{Ri}^{*}=0.85, \mathrm{~d}_{\mathrm{p}}=2.35 \mathrm{~mm} \ldots \ldots \ldots \ldots \ldots \ldots . .97$

Figure 6.9 - Volume fraction contours; $\mathrm{Ri}^{*}=0.85, \mathrm{~d}_{\mathrm{p}}=2.35 \mathrm{~mm}$. ....................................... 98

Figure 6.10 - Streamlines and volume fraction contours; $\mathrm{Ri}^{*}=0.51, \mathrm{~d}_{\mathrm{p}}=1.75 \mathrm{~mm} \ldots \ldots \ldots \ldots \ldots \ldots . .99$

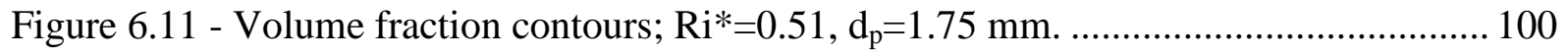

Figure 6.12 - Streamlines and volume fraction contours; $\mathrm{Ri}^{*}=0.51, \mathrm{~d}_{\mathrm{p}}=2.35 \mathrm{~mm} \ldots \ldots \ldots \ldots \ldots \ldots . .101$

Figure 6.13 - Volume fraction contours; $\mathrm{Ri}^{*}=0.51, \mathrm{~d}_{\mathrm{p}}=2.35 \mathrm{~mm}$................................... 102

Figure 6.14 - Instantaneous mixing layer near water inlet; After Wu and Katz [38]............. 104

Figure 6.15 - Instantaneous mixing layer near central section; After Wu and Katz [38]...... 105

Figure 6.16 - Instantaneous mixing layer near fuel inlet diffuser; After Wu and Katz [38]. 106

Figure 6.17 - Typical two-dimensional mixing layer. .......................................................... 109

Figure 6.18 - Gradient Richardson number profiles for case 1........................................... 112

Figure 6.19 - Gradient Richardson number profiles for case 2. .......................................... 112

Figure 6.20 - Gradient Richardson number profiles for case 3........................................... 113

Figure 6.21 - Gradient Richardson number profiles for case 4. .......................................... 113 
Figure 6.22 - Maximum gradient Richardson number versus x/L....................................... 114

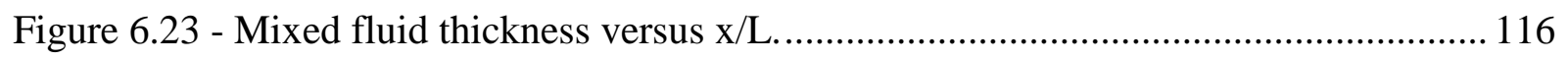

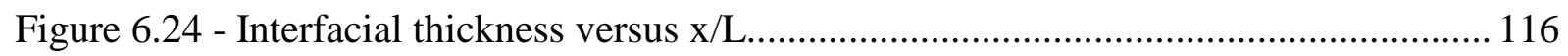

Figure 7.1 - Geometry of CFBT model that was simulated in CFX..................................... 120

Figure 7.2 - Schematic of the computational grid near the inlet. ........................................ 122

Figure 7.3 - Transverse plane showing grid near inlet compartment. ................................... 123

Figure 7.4 - Fuel volume fraction contours near inlet after 5.0 seconds. ............................... 126

Figure 7.5 - Fuel volume fraction contours and velocity vectors after 5.0 seconds. .............. 126

Figure 7.6 - Fuel volume fraction contours and velocity vectors after 15.0 seconds............. 127

Figure 7.7 - Fuel volume fraction contours and velocity vectors after 25.0 seconds. ............ 127

Figure 7.8 - Wireframe schematic of CFBT highlighting manholes and internal openings... 129

Figure 7.9 - Flow rates of fuel and water through manhole 1........................................... 130

Figure 7.10 - Flow rates of fuel and water through manhole 2. ........................................... 130

Figure 7.11 - Flow rates of fuel and water through manhole 3 ........................................... 131

Figure 7.12 - Flow rates of fuel and water through manhole 4........................................... 131

Figure 7.13 - Longitudinal volume fraction profiles in rows 1 and 3 after 100.0 seconds..... 133

Figure 7.14 - Longitudinal volume fraction profiles in rows 1 and 3 after 200.0 seconds..... 134

Figure 7.15 - Longitudinal volume fraction profiles in rows 2 and 4 after 100.0 seconds..... 136

Figure 7.16 - Longitudinal volume fraction profiles in rows 2 and 4 after 200.0 seconds..... 137

Figure 7.17 - Transverse volume fraction profiles after 100.0 seconds. ............................... 138

Figure 7.18 - Transverse volume fraction profiles after 200.0 seconds. ............................... 139 


\title{
Nomenclature
}

\author{
English \\ b Buoyancy \\ B Buoyancy flux \\ $\mathrm{C}_{\mathrm{D}} \quad$ Drag coefficient for a sphere or droplet \\ d Lower layer depth in SL experiments (also mean interface position) \\ $\mathrm{d}_{\mathrm{p}} \quad$ Fluid droplet diameter \\ E Dimensionless entrainment velocity \\ $\mathrm{Fr}_{\mathrm{i}} \quad$ Internal Froude number \\ g Gravitational acceleration \\ $\mathrm{h}_{\mathrm{s}} \quad$ Maximum velocity gradient thickness \\ $\mathrm{h}_{\rho} \quad$ Maximum density gradient thickness \\ $\mathrm{h}_{0} \quad$ Lower concentration boundary layer thickness \\ $\mathrm{h}_{1} \quad$ Upper concentration boundary layer thickness \\ $\mathrm{H} \quad$ Total depth of upper and lower layers in shear flow \\ $\mathrm{H}_{0} \quad$ Lower layer vertical inlet height \\ $\mathrm{H}_{1} \quad$ Upper layer vertical inlet height \\ $\mathrm{H}_{2} \quad$ Lower layer vertical outlet height \\ $\mathrm{H}_{3} \quad$ Upper layer vertical outlet height \\ $\mathrm{k}_{\mathrm{S}} \quad$ Average roughness height \\ $k \quad$ Specific turbulence kinetic energy \\ L Horizontal length of shear layer
}




\begin{tabular}{ll}
$l$ & Turbulence length scale \\
$\mathrm{p}$ & Pressure \\
$\mathrm{P}$ & Modified pressure for turbulent flows \\
$\mathrm{Q}$ & Volumetric flow rate \\
$\mathrm{Q}_{\mathrm{O}}$ & Lower layer inlet flow rate per unit length \\
$\mathrm{Q}_{1}$ & Upper layer inlet flow rate per unit length \\
$\mathrm{Q}_{2}$ & Lower layer outlet flow rate per unit length \\
$\mathrm{Q}_{3}$ & Upper layer outlet flow rate per unit length \\
$\mathrm{r}$ & Volume fraction \\
$\mathrm{Re}_{\mathrm{P}}$ & Particle or droplet Reynolds number \\
$\mathrm{Ri}_{\mathrm{F}}$ & Flux Richardson number \\
$\mathrm{Ri}_{\mathrm{G}}$ & Gradient Richardson number \\
$\mathrm{Ri}_{\mathrm{L}}$ & Mixed layer Richardson number \\
$\mathrm{Ri}_{\mathrm{S}}$ & Shear Richardson number \\
$\mathrm{Ri}^{*}$ & Overall Richardson number \\
$\mathrm{Ri}_{1}$ & Layer Richardson number \\
$\mathrm{Ri}_{\mathrm{O}}$ & Lower layer Richardson number \\
$\mathrm{Ri}_{1}$ & Upper layer Richardson number \\
$\widetilde{\mathrm{u}}$ & Instantaneous velocity \\
$\mathrm{u}$ & Mean velocity \\
\hline & Mean mixture velocity \\
\hline & Velocity fluctuation from mean \\
\hline & Turbulent velocity scale \\
\hline &
\end{tabular}




$\begin{array}{ll}\mathrm{u}_{\mathrm{e}} & \text { Entrainment velocity } \\ \mathrm{u}_{\mathrm{i}} & \text { Mean interface velocity } \\ \mathrm{u}_{\mathrm{S}} & \text { Slip or relative velocity between phase } \alpha \text { and phase } \beta \\ \mathrm{U} & \text { Horizontal free stream velocity } \\ \mathrm{U}^{+} & \text {Dimensionless wall velocity } \\ \mathrm{v} & \text { Mean vertical velocity } \\ \mathrm{v}^{\prime} & \text { Vertical velocity fluctuation from mean }\end{array}$

Greek Symbols

$\begin{array}{ll}\Delta & \text { Interfacial offset } \\ \rho & \text { Density } \\ \rho_{\mathrm{m}} & \text { Mixture density } \\ \rho_{\mathrm{O}} & \text { Lower layer unmixed density } \\ \rho_{1} & \text { Upper layer unmixed density } \\ \delta & \text { Interfacial thickness } \\ \delta_{\mathrm{M}} & \text { Mixed fluid thickness } \\ \delta_{\mathrm{S}} & \text { Shear layer thickness } \\ \kappa & \text { Von Karman Constant } \\ \eta & \text { Mean interface position } \\ \mu & \text { Dynamic viscosity } \\ \mu_{\mathrm{m}} & \text { Mixture viscosity } \\ \mu_{\mathrm{T}} & \text { Eddy viscosity }\end{array}$




$\begin{array}{ll}\Gamma & \text { Diffusivity } \\ \Gamma_{\mathrm{T}} & \text { Eddy diffusivity } \\ \varepsilon & \text { Dissipation of specific turbulence kinetic energy } \\ \sigma & \text { Surface tension }\end{array}$

Subscripts

$\alpha \quad$ Phase alpha (denotes lighter phase)

$\beta \quad$ Phase beta (denotes heavy phase)

$\mathrm{m} \quad$ Indicates mixture quantity

1 Indicates quantity from upper layer in homogeneous shear flow

0 Indicates quantity from lower layer in homogeneous shear flow 


\subsection{INTRODUCTION}

\subsection{Background}

Compensated fuel/ballast tanks (CFBT) are used in US Navy ships for stability and ease of operation. The tanks, which are located in the bottom of ships, are linked together in groups of 2-5 tanks [3]. A sketch of a typical compensated fuel/ballast tank, which is referred to as DDG 51 Tank 5-300-2-F CFBT, is shown in Figure 1.1.

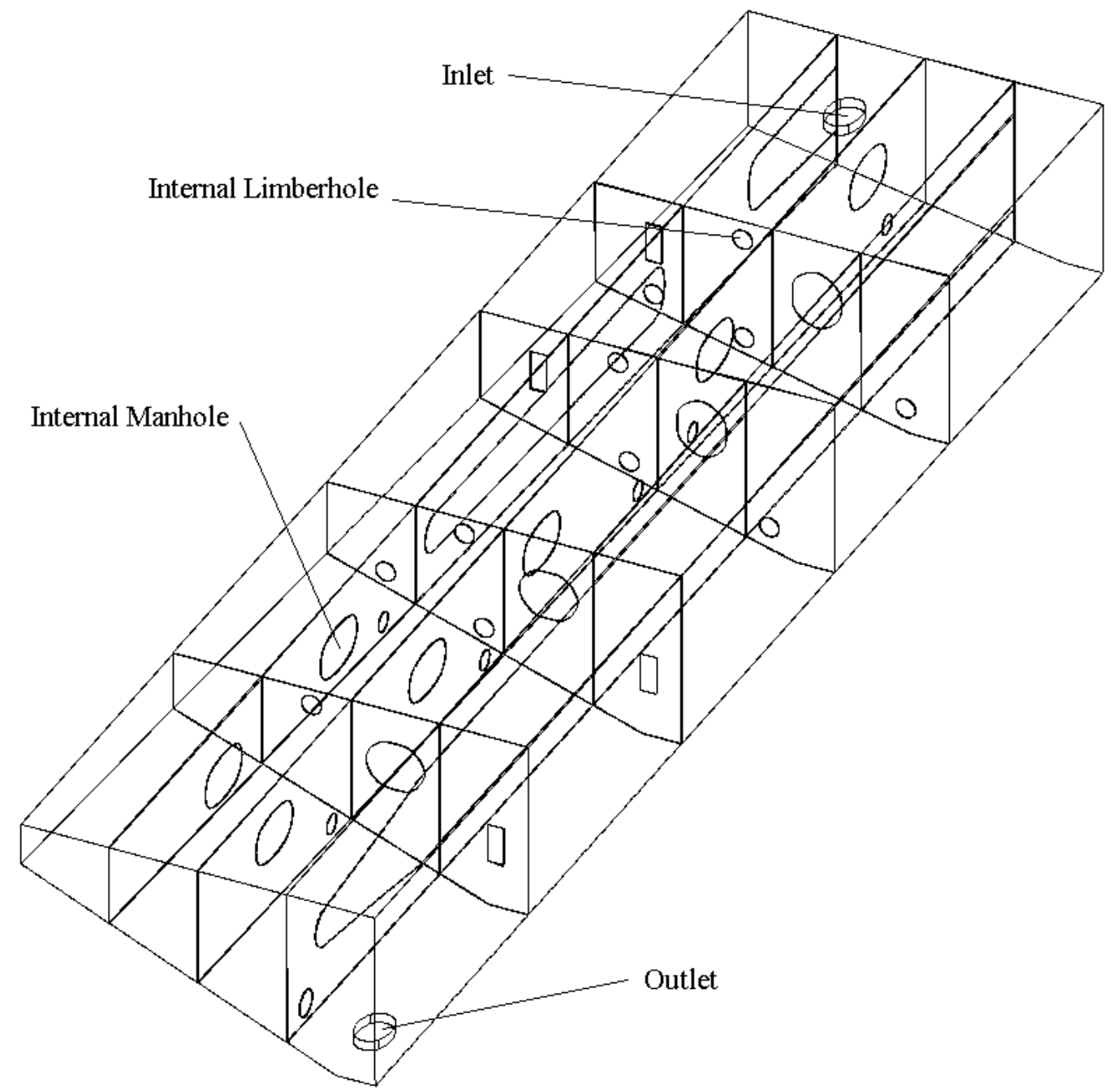

Figure 1.1 - Geometry of baseline DDG 51 Tank 5-300-2-F CFBT. 
As shown in Figure 1.1, the typical tank is broken up into a series of compartments that are connected by manholes and limberholes. Each tank may hold anywhere from 30,000 to 120,000 liters (8,000 to 32,000 gallons) of fuel, with each set of tanks holding approximately 260,000 liters (70,000 gallons). The typical compensated fuel/ballast tank that is shown in Figure 1.1 is $12.0 \times 2.7 \times 1.4$ meters, where the tank is 1.4 meters high at the highest point. As fuel is drawn off the top, sea water is allowed to enter the bottom of the tanks and when empty a tank typically has a $0.08 \mathrm{~m}$ (3.0 in) layer of fuel on the top. During a typical re-fueling process, fuel enters near the top of the tank, through an upward inlet pipe, at flow rates between 60 to 95 liters/sec (950 to 1,500 gal $/ \mathrm{min}$.). The compensating water is forced out of the bottom of the tank through an outlet, located in the last tank. The density of the fuel is about $850 \mathrm{~kg} / \mathrm{m}^{3}$, compared to the density of the sea water which is approximately $1,025 \mathrm{~kg} / \mathrm{m}^{3}$. Though, in general, buoyancy forces compete to keep the fluids separated, the turbulence that develops because of the relatively high shear rates and the internal obstacles causes some of the fuel to become entrained in the sea water that is discharged overboard. This is a concern not only because of the wasted fuel but primarily because of the possible violation of environmental regulations. Another problem typically encountered in these tanks is that of water hideout, where sea water is trapped at the bottom of a compartment. This has been estimated to happen in up to $20 \%$ of the tank and is an obvious inefficiency [3].

A common flow scenario characterizing the re-fueling of a CFBT is a high shear flow that develops at the interface of the fuel and water. This happens as the fuel pushes the water out of the bottom of the tank. In light of this flow scenario, the mechanisms that 
influence mixing at a sheared, density stratified interface are important in both understanding and controlling the flow through a compensated fuel/ballast tank. One important characteristic of the shear flow that occurs in the tank is that the fuel and water are immiscible fluids. Most experimental measurements involving density stratified, shear flows involve miscible fluids (e.g. fresh water and salt water) and only investigate the mixing and transport of some scalar quantity, usually salt concentration or heat. While in general the behavior of immiscible and miscible fluids is fundamentally different, some aspects of shearing flows involving miscible fluids are applicable to characterizing and understanding similar flows involving immiscible fluids. Therefore the present study is undertaken with the goal of investigating density stratified, shearing flows involving both miscible fluids and immiscible fluids.

\subsection{Objectives}

The long term goal of this study, is the development of a computational tool that can be used to predict fuel concentration in the compensating water. The predictions from this computational model could be used to suggest structural and operational modifications that can reduce the amount of fuel that is discharged overboard as well as reducing the water hideout [3]. This work was jointly undertaken by researchers and engineers at the Naval Surface Warfare Center (NSWC) in Bethesda, Maryland, at Johns Hopkins University in Baltimore, Maryland, and at West Virginia University (WVU). This study is part of the larger scope of work at WVU that is being performed under the direction of Dr. Ismail Celik and involves the development of a multiphase model for turbulent flows, suitable for predicting fuel entrainment during the re-fueling of a compensated fuel 
ballast tank. In light of the overall project, the work contained in this thesis centers around three objectives. The first objective involves the simulation of density stratified, shearing flows involving two miscible fluids. These simulations investigate how accurately certain multiphase models and turbulence models predict trends in flow parameters that are observed experimentally. The variation of key parameters such as the interfacial thickness and characteristic Richardson numbers, as well as any production or dissipation of turbulent kinetic energy due to buoyancy effects, is also investigated in these simulations.

The second objective involves the simulation of mixing of immiscible fluids in a developing, density stratified, mixing layer. In addition to predicting experimentally observed trends in these simulations, methods for simulating relative velocities between the fluids is investigated. Here, the relative motion of the fluids is important for capturing settling and rise velocities of the fluid droplets that form at or near the sheared interface. Effects of drag, buoyancy, gravity, and surface tension are some of the important parameters in flows involving immiscible fluids that mix, and methods for accounting for such effects are investigated. Characteristic Richardson numbers are also considered in these simulations, as well as effects of the droplet sizes that form in the shear layer.

The third objective deals with simulating the transient filling of a full scale, compensated fuel/ballast tank, based on the multiphase modeling information that is gained from the two-dimensional simulations involving the shear layers. These simulations involving the 
CFBT are used to evaluate the performance of various multiphase models on complicated geometries and to investigate aspects of the flow inside the tank that are related to

mixing. Some of the areas of interest in these simulations are where the most mixing takes place in the tank and what the re-fueling efficiency is for the CFBT that was simulated. The fueling efficiency compares the time that it takes for the fuel to reach the outlet compared to the mean flow through time.

In all of the simulations, the effectiveness of using a single-fluid, mixture model is also investigated. Here, only one set of momentum equations is solved along with an additional scalar equation that is solved for the volume fraction of the fuel. The reason for using the single-fluid, mixture model, is to provide computational efficiency. This is necessary as some of the numerical ballast tank simulations require at least 250,000 computational cells and computer simulations can take weeks.

\subsection{Overview}

First, the literature on turbulence generation and mixing in density stratified, shear flows is reviewed. An introduction to the general parameters of interest that are used to describe stratified shear flows is given along with experimental findings and descriptions from several of the selected papers. Current literature on numerical simulations of density stratified shear flows is also discussed.

Following the literature review, a general introduction to the multiphase flow models that were used to predict the mixing of two liquids is reviewed. Namely, two variations of a 
single fluid, mixture model referred to as the SFST model are discussed. The SFST model is a simplified variation of Ishii's drift flux model [16]. Turbulence models for single phase flows are presented along with the methods and assumptions used to account for buoyant production and dissipation of turbulent kinetic energy in multiphase flows. This discussion of the multiphase models and turbulence models lays the groundwork for the simulations involving the shear flows and the compensated fuel/ballast tank.

Following the review of the models, several numerical simulations involving a series of experiments investigating the mixing and transport of a scalar at a sheared density interface involving water and brine are presented. These experiments were recently conducted by Sullivan and List [28]. Here, the version of the SFST model that is used to simulate the mixing is well suited for miscible fluids and is referred to as SFST1. This model is used in light of the fact that these experiments involving miscible fluids are essentially related to the distribution of a scalar defining the concentration or volume fraction of one of the fluids.

Next a series of simulations characterizing the mixing of two immiscible liquids in a density stratified mixing layer are performed. The multiphase model used for these simulations is a form of the SFST model that is well suited for flows involving immiscible fluids and is referred to as SFST2. Results of the numerical simulations are discussed in light of experimental observations involving single phase mixing layers and density stratified shear layers. Methods for modeling the droplet breakup and formation 
during the shearing of the immiscible fluids is also discussed, as well as the methods used for handling relative phase velocities.

Considering the time averaged solutions that were obtained for the cases involving density stratified, shear layers, some clarification should be made in regards to the purpose of the simulations. For cases involving density stratified, shear layers, it is possible to predict Kelvin-Helmholtz waves, which are an inviscid phenomena and which can be provoked to occur in a numerical simulation by imposing a disturbance. This disturbance will lead to flow instabilities and eventually to the stable mode of the KelvinHelmholtz waves. The prediction of Kelvin-Helmholtz waves requires an appropriate grid size, relatively small time steps, and appropriate boundary conditions. However, the goal of the simulations in the current study was to predict the time averaged flow field and the time averaged effects of such occurrences as Kelvin-Helmholtz waves, not the Kelvin-Helmholtz flow structures themselves. It should also be mentioned that the use of such models as the $k$ - $\varepsilon$ turbulence model, which by design increases the apparent viscosity and diffusivity of the flow, would make the numerical prediction of KelvinHelmholtz structures difficult in any simulation. Such quantities as the gradient Richardson number were calculated with the goal of identifying the mixing regimes and mixing mechanisms that the predicted flow field presumably represented appropriately in a time averaged sense.

Finally, a transient, three dimensional simulation of a compensated fuel/ballast tank is performed using SFST2 and a form of the $k-\varepsilon$ turbulence model that is applicable to 
buoyant flows. The discussion focuses on the applicability of using SFST2 for the immiscible flow simulations in a realistic geometry as is encountered in a CFBT. Several key observations related to the flow inside a fuel ballast tank are presented where areas of high mixing and the fueling efficiency are discussed in light of the given geometry.

As a general note, it should be understood that in this work the term "two-phase flow" refers to two distinct liquids, rather than two different phases. This interpretation is somewhat more general than the typical thermodynamic interpretation involving solids, liquids, and gases; however, it is common practice in the literature when describing any flow with more than one unique fluid to use the term "two-phase flow". Also, a variable referred to as the volume fraction, defined as the volume of the particular phase per unit volume, is used extensively in two-phase theory and modeling. In the experiments involving miscible fluids the term concentration is used by the experimenters to describe a variable that is similar to the volume fraction. Hence, the terms volume fraction and concentration will be used interchangeably in light of the miscible shear flow experiments. 


\subsection{REVIEW OF LITERATURE}

\subsection{Introductory Definitions and Concepts}

Buoyant flows, specifically density stratified, shear flows, are characterized by a common set of parameters and dimensionless numbers. The primary distinction of a buoyant flow from other flows is that the effects to be described result from the action of some body force, usually gravity, on variations in the density from point to point in the flow [33]. Before presenting a somewhat detailed review of the literature dealing with density stratified shear flows, some description of the parameters and definitions characterizing these flows is made. This will serve to better clarify the results presented from the literature and to give some introduction to the parameters that are of interest in the numerical simulations. An idealized shear flow scenario is shown in Figure 2.1, illustrating some of the parameters that are of interest. The parameters described are understood to apply to incompressible fluids, where gravity is the only body force.

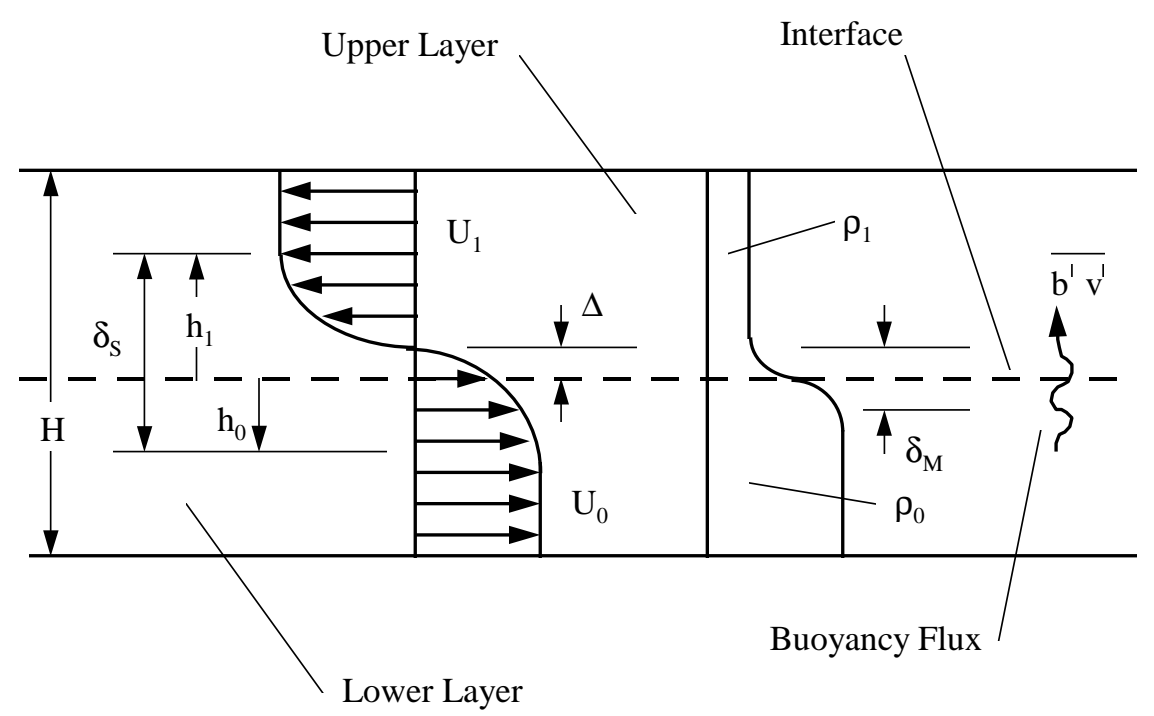

Figure 2.1 - Idealized density stratified shear flow. 
Density variations in a fluid give rise to some departure from hydrostatic equilibrium and can be characterized by the reduced gravitational force that the fluid element will feel in the event of a density fluctuation. A parameter describing this reduced gravitational force or buoyancy force can be defined by the density variation from some reference density. Hence a term commonly found in the literature describing buoyant flows is given by

$$
\mathrm{b}=\mathrm{g} \frac{\rho-\rho_{1}}{\rho_{1}}
$$

where $\rho_{1}$ is usually the density of the lighter fluid. This term is commonly referred to as the buoyancy term, which is essentially a buoyant force per unit mass.

A key parameter characterizing the extent of mixing at the interface of a density stratified flow, is the entrainment velocity [28], designated as $\mathrm{u}_{\mathrm{e}}$. For a transient stratified, shear flow, the entrainment velocity is defined as the normal velocity at the edge of the mixed fluid, as the width of the mixed fluid thickness, $\delta_{\mathrm{M}}$, grows with time [23]. For a steady state situation the entrainment velocity is defined as the volumetric flow rate of the fluid being entrained, divided by the cross sectional area over which entrainment is occurring [23]. This amounts to determining the volume of lower layer fluid that is entrained in the upper layer per unit time per unit area [28]. This parameter may also be regarded as a time rate of change of potential energy per unit mass [23]. The dimensionless entrainment velocity is usually given by

$$
\mathrm{E}=\frac{\mathrm{u}_{\mathrm{e}}}{\mathrm{U}_{\mathrm{ch}}}
$$


where $U_{c h}$ is a characteristic velocity scale associated with either the free stream or some characteristic shear stress. $\mathrm{E}$ is also referred to as the dimensionless entrainment.

Another key parameter characterizing the extent of mixing at the interface is the turbulent buoyancy flux which is given by

$$
B=g \frac{\overline{\left(\rho^{\prime}-\rho_{1}\right) v^{\prime}}}{\rho_{1}}=g \frac{\overline{\rho^{\prime} v^{\prime}}}{\rho_{1}}=\overline{v^{\prime} b^{\prime}}
$$

where the overbar indicates a time averaged quantity (Note that $\rho_{1}$ is constant but the density $\rho$ is not). In some cases this parameter appears in the turbulent kinetic energy equation and in many cases is found to be of the same order of magnitude as the viscous dissipation of turbulent kinetic energy at a density stratified, sheared interface. The buoyancy flux may also be regarded as a time rate of change of potential energy per unit mass and can be related to $\mathrm{u}_{\mathrm{e}}$.

In a homogeneous, density stratified shear flow, primarily two vertical length scales characterize the shear layer. Typically these two scales are taken to be either the shear layer thickness, $\delta_{\mathrm{S}}$, and the mixed fluid thickness, $\delta_{\mathrm{M}}$, (See Figure 2.1) or the maximum velocity gradient thickness, $h_{S}$, and the maximum density gradient thickness $h_{\rho},[1] . h_{S}$ is defined by

$$
\mathrm{h}_{\mathrm{s}}=\frac{\left(\mathrm{U}_{1}-\mathrm{U}_{0}\right)}{(\partial \mathrm{u} / \partial \mathrm{y})_{\mathrm{Max}}}
$$

where $(\partial \mathrm{u} / \partial \mathrm{y})_{\text {Max }}$ is the maximum vertical velocity gradient at the interface. $\mathrm{h}_{\mathrm{s}}$ may be thought of as a length scale that is inversely proportional to the shear rate. In terms of a 
laminar Couette flow between parallel plates that are separated by a distance $h_{S}$ and moving with opposite velocities $U_{1}$ and $U_{0}, \partial u / \partial y$ would equal $\left(U_{1}-U_{0}\right) / h_{S}$. In this case a small $h_{S}$ indicates a higher shear. The maximum density gradient thickness is given by

$$
h_{\rho}=\frac{\left(\rho_{0}-\rho_{1}\right)}{(\partial \rho / \partial y)_{\max }}
$$

and represents a parameter characterizing the extent of the buoyancy forces at the interface, similar to that defined for the velocity [1]. A small $h_{\rho}$ would indicate a relatively steep density gradient or a more buoyancy dominated flow compared to a large $h_{\rho}$. The ratio given by $h_{S} / h_{\rho}$ is sometimes used as a relative measure of the influences of buoyancy and turbulence. This ratio gives a parameter that is somewhat similar to the gradient Richardson number. For a case governed by linear gradients, $\delta_{\mathrm{S}}$ and $\delta_{\mathrm{M}}$ are equal to $h_{S}$ and $h_{\rho}$ respectively. Another length scale that is used in some of the literature is the interfacial thickness, $\delta$, which is different than $\delta_{\mathrm{M}}$. Typically $\delta$ is defined in terms of the mixing in the vicinity of the interface and $\delta_{M}$ represents the entire layer over which mixing occurs. More formal definitions of these two parameters will be given in later chapters.

Literally all buoyant flows, whether stratified shear flows or not, rely on a fundamental parameter that characterizes, to some degree, the relative influence of buoyancy to inertia or buoyancy to turbulence. This parameter is the Richardson number, of which many definitions exist depending on the characteristics of the flow and the phenomena in question. Six of the most common definitions of this parameter and their interpretations for stratified shearing flows, will be presented here. This will provide some basis for 
comparing the results presented in the literature that was investigated, and in describing the numerical simulations. Namely, descriptions for the overall, gradient, shear, layer, mixed layer, and flux Richardson numbers will be presented.

Density stratified shearing flows are characterized by several mixing regimes that develop at the interface based on what instabilities are present. Depending on the relative magnitudes of $\delta_{\mathrm{S}}$ and $\delta_{\mathrm{M}}$ or $\mathrm{h}_{\mathrm{S}}$ and $\mathrm{h}_{\rho}$, several kinds of instabilities and mixing mechanisms are observed at the interface [10]. Typically some ratio giving the relative influence of the vertical shear scale to vertical density scale, may be formed and the mixing regimes are then characterized by the resulting dimensionless number. Two of these dimensionless numbers are the gradient and shear Richardson numbers.

The gradient Richardson number is generally regarded as a measure of the local instability at the mixed interface, between the shear layers, and is defined by

$$
\mathrm{Ri}_{\mathrm{G}}=\frac{-(\mathrm{g} / \rho)(\partial \rho / \partial \mathrm{y})}{(\partial \mathrm{u} / \partial \mathrm{y})^{2}}
$$

Tennekes and Lumley regard this parameter as a ratio of the local mean shear time scale to the mean buoyant time scale [29]. This implies that the buoyancy will tend to respond to turbulent fluctuations on a much faster rate and quickly damp interfacial mixing as the gradient Richardson number increases. In this regard $\mathrm{Ri}_{\mathrm{G}}$ could be thought of as some measure of the time it takes a turbulent fluctuation to penetrate the interface divided by the time it takes the buoyancy to eject any fluid of differing density caused by the turbulent fluctuations. 
Another parameter which gives some overall measure of the interfacial instabilities in a turbulent shear flow is the shear Richardson number given by

$$
\mathrm{Ri}_{\mathrm{S}}=\frac{\mathrm{bh}_{\mathrm{S}}}{\left(\mathrm{U}_{0}-\mathrm{U}_{1}\right)^{2}}
$$

for layer free stream velocities $\mathrm{U}_{0}$ and $\mathrm{U}_{1}$. Here $\mathrm{h}_{\mathrm{S}}$ is the maximum velocity gradient thickness. The interpretation of $\mathrm{h}_{\mathrm{S}}$ previously given carries into $\mathrm{Ri}_{\mathrm{S}}$ as a relatively small $\mathrm{h}_{\mathrm{S}}$ would cause $\mathrm{Ri}_{\mathrm{S}}$ to be low indicating a more turbulent dominated interface, and a larger $h_{S}$ would indicate a more buoyancy dominated interface. This parameter is useful in that it gives a single overall characterization to the relative influence of shear and buoyancy for a homogeneous, stratified shear flow.

As there are usually two turbulent layers in a stratified flow, the layer Richardson number gives some measure of the buoyancy and turbulence influences in the stratified layers themselves. Here the relative length scale for either stratified layer is usually defined to be the partial shear layer extending from the center of the mixed interface to the point above or below the interface where no velocity gradient exists. This is illustrated in Figure 2.1. The scale of the shear layer plays an important role in the entrainment process, as it is responsible for the production of turbulent kinetic energy at the interface [24]. With the free-stream velocity characterizing the shear, the layer Richardson number is given by

$$
\mathrm{Ri}_{1}=\frac{\mathrm{hb}}{\left(\mathrm{U}-\mathrm{u}_{\mathrm{i}}\right)^{2}}
$$


for either the top or bottom layer. Here $\mathrm{h}$ is the thickness of the momentum or concentration boundary layer and $u_{i}$ is the interface velocity and is used to give some measure of the shear as taken in a reference frame moving with the interface. This is the primary reason that the Richardson number for two layers based on the relative shear with respect to the interface velocity may be quite different than that estimated by assuming the interface occurred at the point of zero velocity. Since the interfacial offset, $\Delta$, can be non-zero it is possible for the instability mechanisms to diverge into different modes, one characterizing the lower layer and one characterizing the upper layer, depending on the relative gradients in each layer [10]. This is equivalent to having one set of buoyancy and turbulence length scales in the bottom layer and another set of scales in the upper layer, necessitating two Richardson numbers for characterizing entrainment.

A slightly different form of the layer Richardson number is also frequently used and will be referred to as the mixed layer Richardson number. This parameter can be used when two layer Richardson numbers are not desired, but the width of the layer over which mixing occurs is the desired length scale. The mixed layer Richardson number is given by

$$
\mathrm{Ri}_{\mathrm{L}}=\frac{\delta_{\mathrm{M}} \mathrm{b}}{\left(\mathrm{U}_{0}-\mathrm{U}_{1}\right)^{2}}
$$

The mixed fluid thickness is sometimes replaced by the shear layer thickness, $\delta_{\mathrm{S}}$, which may be a more appropriate length scale for certain stratified flows. 
The overall Richardson number is generally used as a parameter that describes the overall state of a stratified flow using some characteristic flow dimension. This characteristic flow dimension that is usually taken as the entire depth of the two layers. Hence this parameter should not be confused with any local parameters based on gradients of density or velocity [34]. The overall Richardson number is defined as

$$
\mathrm{Ri}^{*}=\frac{\mathrm{Hb}}{\left(\mathrm{U}_{\mathrm{ch}}\right)^{2}}
$$

where $\mathrm{U}_{\mathrm{ch}}$ is some characteristic velocity scale. $\mathrm{U}_{\mathrm{ch}}$ is typically defined as the difference between the lower and upper layer, free stream velocities. Here $\mathrm{H}$ is characteristic of the turbulence for cases where the flow in both layers is turbulent and for cases where the shear at the interface is not entirely related to interfacial length scales. A parameter that is referred to as the densimetric Froude number is sometimes used in place of the overall Richardson number and is given by

$$
\mathrm{Fr}_{\mathrm{i}}=\frac{1}{\sqrt{\mathrm{Ri}^{*}}}
$$

The other definition of the Richardson number is based on the rate of buoyant dissipation to turbulent stress production of turbulent kinetic energy. While this parameter may be defined in several ways, a general definition comes from the turbulent kinetic energy equation. For a horizontally homogeneous flow where $v^{\prime}$ represents the vertical velocity fluctuations and the turbulent Reynolds stresses are characterized by $\rho u^{2}$, which is

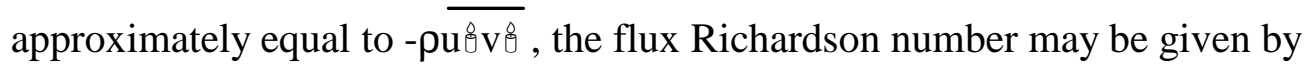

$$
\mathrm{Ri}_{\mathrm{F}}=\frac{\mathrm{g} \overline{\mathrm{v}^{\prime} \rho^{\prime}}}{\rho u^{2} \partial \mathrm{u} / \partial \mathrm{y}}
$$


The flux Richardson number represents the ratio of the rate of removal of turbulent kinetic energy by buoyant forces to the production of turbulent kinetic energy by shear [33]. When the buoyancy term and the production term are modeled by

$$
\mathrm{Ri}_{\mathrm{F}}=\frac{\mathrm{g} \overline{v^{\prime} \rho^{\prime}}}{\rho u^{2} \partial \mathrm{u} / \partial \mathrm{y}} \cong \frac{\mathrm{g}\left(v_{\mathrm{T}} / \sigma_{\rho}\right) \partial \rho / \partial \mathrm{y}}{\rho v_{\mathrm{T}}(\partial \mathrm{u} / \partial \mathrm{y})^{2}}
$$

the flux Richardson number is approximately the same as the gradient Richardson number for cases where the turbulent viscosity and diffusivity are close to equal [29]. Positive values of the flux Richardson number usually indicate stable stratification, as positive fluxes upward of buoyancy generally correspond to stable stratification [29].

In characterizing stratified shear flows, most experiments describe several interfacial mixing regimes that depend on the level of influence of buoyancy and turbulence at the interface. Given some Richardson number that describes the influence of buoyancy and turbulence or shear, these regimes are primarily characterized by the manner in which the interfacial mixing occurs. For very low values of some Richardson number, a regime is defined where mixing is primarily caused by interfacial instabilities that are commonly observed in constant density flows where the interface is fully turbulent. This interface is regarded to show significant Reynolds number dependence as the flow situation is very similar to flows involving no density stratification. As the Richardson number is increased, the turbulence at the interface is damped and the mixing is primarily by Kelvin-Helmholtz type waves, which thicken the interface by periodic formation and breaking. At still larger Richardson numbers, Kelvin-Helmholtz instabilities are no longer observed and the interfacial mixing is driven by interfacial waves which form and 
are sheared off by turbulent eddies; here an eddy from the homogeneous layer, scours the surface of the mixed interface and draws fluid of greater density into a crest, which is then sheared off. At extremely high Richardson numbers, interfacial waves are suppressed and the mixing is dominated by molecular diffusion, which implies a Peclet number dependence as well. Overall it is commonly agreed that four mixing regimes exist [28]. As an added note, the majority of the work in the field seems to have been done in the Kelvin-Helmholtz and wave breaking regimes.

\subsection{Density Stratified, Shear Flow Literature}

Moore and Long [23] performed experiments in a stably stratified layer of two fluids in a cyclically continuous tank of rectangular cross section. Steady state, horizontally homogeneous, shear flow was achieved in these experiments, where salt water or brine was flowing at the bottom layer, and fresh water was flowing at the top layer in the opposite direction. A series of inlet jets and withdrawal slits, which were uniformly distributed at the top and bottom walls, were used to induce the shear flow and to maintain pure, unmixed fluid along the top and bottom walls.

To characterize their experiments Moore and Long defined an overall Richardson number, $\mathrm{Ri}^{*}$ by

$$
\mathrm{Ri}^{*}=\frac{\mathrm{Hb}}{(2 \Delta \mathrm{U})^{2}}
$$

where $\mathrm{H}$ is the total depth and $-0.5 \mathrm{~b}, 0.5 \mathrm{~b}, 0.5 \Delta \mathrm{U}$, and $-0.5 \Delta \mathrm{U}$ are the buoyancies and velocities, respectively, at the top and bottom of the layer. 
Here the buoyancy is given by

$$
\mathrm{b}=\mathrm{g} \frac{\rho_{0}-\rho_{1}}{\rho_{\mathrm{R}}}
$$

where $\rho_{R}$ is the average of the top and bottom layer densities $\rho_{1}$ and $\rho_{0}$, respectively. Experiments were conducted for $\mathrm{Ri}^{*}$ ranging from 1.0 to 30.0 , based on averaged quantities over the stratified layers. The flow regimes considered in this set of experiments consisted of a low Richardson number regime $\left(\mathrm{Ri}^{*}<3.0\right)$ where mixing was predominantly influenced by K-H type of structures, and a higher Richardson number regime $\left(\mathrm{Ri}^{*}>3.0\right)$ where mixing was primarily by shear driven wave breaking. Moore and Long suggested a relationship for the dimensionless entrainment velocity given by

$$
\mathrm{E} \propto \frac{1}{\mathrm{Ri}^{*}}
$$

where $\mathrm{Ri}^{*}$ was defined by Eq. (2.2.1). The thickness of the layer over which the density changes was also inversely proportional to $\mathrm{Ri}^{*}$.

Sullivan and List [28] performed experiments by measuring tracer dye concentrations in two-layer, density stratified, turbulent shear flows. A counter flow was generated by two inlets at the opposite ends of a $5.0 \mathrm{~m}$ long and $20.0 \mathrm{~cm}$ high channel. These experiments were performed over a range of shear Richardson numbers varying from 0.1 to 1.0 , which corresponded to a K-H mixing regime and a shear driven, wave breaking mixing regime. Sullivan and List also studied a mechanism known as eddy scouring, where fluid at the mixed interface is vertically transported into homogeneous layers by large scale turbulent eddies. Sullivan and List seem to be one of the first to specifically discuss the four 
mixing regimes mentioned in Section 2.1. To characterize their flow scenario, Sullivan and List used the layer Richardson number, given as

$$
\mathrm{Ri}_{1}=\frac{\mathrm{hb}}{\left(\mathrm{U}-\mathrm{u}_{\mathrm{i}}\right)^{2}}
$$

which was described in Section 2.1. A buoyancy term similar to that of Moore and Long's was defined by

$$
\mathrm{b}=\mathrm{g} \frac{\rho_{0}-\rho_{1}}{\rho_{1}}
$$

for the top and bottom layer densities $\rho_{1}$ and $\rho_{0}$, respectively. Sullivan and List also used the shear Richardson number given by

$$
\mathrm{Ri}_{\mathrm{S}}=\frac{\mathrm{bh}_{\mathrm{S}}}{\left(\mathrm{U}_{0}-\mathrm{U}_{1}\right)^{2}}
$$

as a parameter that is generally regarded as characterizing the interfacial instabilities in a turbulent shear flow as is outlined in Section 2.1, along with the definition of $h_{s}$. The experimental observations indicated that the dimensionless mixed interface thickness, $\delta / h$, as defined by Sullivan [28] and the dimensionless entrainment velocity, E, could be expressed in a functional form of

and

$$
\begin{aligned}
& \delta / \mathrm{h} \propto \mathrm{Ri}_{1}{ }^{-1 / 2} \\
& \mathrm{E} \propto \mathrm{Ri}_{1}{ }^{-3 / 2}
\end{aligned}
$$

in the $\mathrm{K}-\mathrm{H}$ regime and by

and

$$
\begin{aligned}
& \delta / \mathrm{h} \propto \mathrm{Ri}_{1}{ }^{-1.0} \\
& \mathrm{E} \propto \mathrm{Ri}_{1}{ }^{-2.0}
\end{aligned}
$$

in the wave breaking regime. This was determined to be true for cases where only one of the layers was turbulent and entraining. The dependence of the Reynolds number was 
found to be unimportant except in the regime of extremely low Ri, where the interface is fully turbulent. Peclet number dependence was found to exist in the molecular diffusion dominated regime for high $\mathrm{Ri}_{1}$. When both layers are turbulent, it was found that appropriate combinations of the layer Richardson numbers and other parameters must be used for appropriate correlations. Based on the experimental study, a model was developed where $\delta$ and $\mathrm{E}$ were functions of the combined layer Richardson numbers.

Atsavapranee and Gharib [1] studied a stratified, plane mixing layer using a $2.4 \mathrm{~m}$ long by $13.0 \mathrm{~cm}$ high, stratified tilting tank. These experiments dealt primarily with identifying and describing structures and secondary instability mechanisms as functions of the shear Richardson number between 0.0 and 0.25 . Effects of the two vertical length scales, $h_{S}$ and $h_{\rho}$, was also investigated. In addition to the primary Kelvin-Helmholtz instabilities typically observed at lower $\mathrm{Ri}_{\mathrm{S}}$, secondary instabilities were also described. Primarily, upon formation of a Kelvin-Helmholtz structure, smaller Kelvin-Helmholtz structures within the larger vortex were observed that enhance the mixing at the interface. Gravitational instabilities due to the variations in density were also observed to occur in the core of the Kelvin-Helmholtz structure and increase the amount of mixing at the interface. The roll up and pairing of the Kelvin-Helmholtz vortices is also illustrated.

Narimousa and Fernando [24] performed experiments of a shear flow where a lighter layer was driven over a heavy quiescent layer in a closed loop water channel. Here an overall Richardson number defined by

$$
\mathrm{Ri}^{*}=\frac{\mathrm{Hb}}{\mathrm{U}^{2}}
$$


was used, where $\mathrm{H}$ was the vertical distance from the mixed interface to the top of the upper layer, and $U$ was the average velocity in the upper layer, which was fully turbulent. $\mathrm{U}$ was essentially the velocity jump across the mixed interface. These experiments were performed over a range of overall Richardson numbers, $\mathrm{Ri}^{*}$, varying from 0.0 to 25.0, which corresponded to a $\mathrm{K}-\mathrm{H}$ mixing regime and a wave breaking mixing regime. The $\mathrm{K}-\mathrm{H}$ regime was observed to persist for $0.0<\mathrm{Ri}^{*}<5.0$, followed by the wave breaking regime for $5.0<\mathrm{Ri}^{*}<20.0$. At still higher overall Richardson numbers, molecular effects were observed to be important. In these experiments, a trend of the normalized entrainment velocity followed

$$
\mathrm{E} \propto \frac{1}{\mathrm{Ri}^{*}}
$$

where $\mathrm{u}_{\mathrm{e}}$ was normalized by the average velocity in the upper layer in calculating $\mathrm{E}$. Estimates of the buoyancy flux and production and dissipation of turbulent kinetic energy at the mixed interface showed all three quantities to be of the same order. Some evidence was also given for the validity of using a mean flow gradient-transport model in describing the buoyancy flux, in homogeneous shear flows.

Fernando [10] gives an overall review of the majority of the experiments performed in stratified shear layers, discussing reported mixing mechanisms, entrainment laws, and the characteristic types of laboratory shear flows. For a two layer density stratified shearing flow, where the primary generation of turbulence takes place at the interface, Fernando gives the governing parameters as a type of combined layer Richardson number given by

$$
\mathrm{Ri}_{\mathrm{L}}=\frac{\delta_{\mathrm{S}} \mathrm{b}}{\left(\mathrm{U}_{0}-\mathrm{U}_{1}\right)^{2}}
$$


the normalized interfacial displacement given by

$$
\mathrm{L}_{\mathrm{Ch} 1}=\frac{2 \Delta}{\delta_{\mathrm{S}}}
$$

and the ratio of the buoyancy and velocity interfacial thickness given by

$$
\mathrm{L}_{\mathrm{Ch} 2}=\frac{\delta_{\mathrm{M}}}{\delta_{\mathrm{S}}}
$$

This is similar in some sense to using two layer Richardson numbers to characterize the entrainment and mixing regimes and the shear Richardson number to characterize the instability mechanisms. Fernando describes the typical entrainment formulation given by

$$
\mathrm{E} \propto \frac{1}{\mathrm{Ri}_{\mathrm{L}}}
$$

as coming from a balance of the rate of change of potential energy to the kinetic energy flux at the interface [10].

Turner and Ellison [33] discuss the typical instability and mixing mechanisms already described as well as giving some extensive arguments based on the governing turbulent kinetic energy and buoyancy (mass) equations. By considering the three fluctuating velocity components separately, the influence of the buoyancy term is discussed in light of its effects on dissipating the turbulence and its direct influence in making the turbulence non-isotropic. The possibilities of applying an equilibrium based theory are also discussed in light of a longitudinally homogeneous flow, where the shear production is balanced by the viscous and buoyant dissipation [33]. 
Overall the literature reviewed indicates that there is no agreement on which Richardson numbers should be used for the different shear flows. It is clear that the turbulence should scale with its primary production mechanism and that two layer flows may exhibit different mixing regimes depending on the offset of the shear and concentration interfaces. The form of an entrainment relation is another parameter that is not certain, though it is expected to go as $\mathrm{E} \sim \mathrm{Ri}^{-\mathrm{n}}$. Several mixing regimes are identifiable and it is agreed that the power law dependency of the Richardson number will change from one regime to the next. Overall no complete model for entrainment has been formulated, though much progress has been made in understanding and predicting certain classes of flows.

\subsection{Brief Review of Computational Studies}

In light of the extensive work that has been done on the experimental side, the progress in analytically or computationally simulating density stratified, shear flows is also reviewed. This area is also quite extensive in quantity, and several important contributions have been chosen for review.

Turner [33] reviews one of the first mathematical treatments of the problem, as given by Townsend [30], by considering the equations for the turbulent kinetic energy and mean square density fluctuations in a stratified, homogeneous shear flow. Neglecting small diffusion terms in the turbulent kinetic energy equation, the $k$-equation reduces to a balance between production by the Reynolds stress and the dissipation by working against buoyancy gradients and viscous dissipation. In a similar manner, the equation governing 
the density fluctuations is shown to reduce to terms representing the balance between production and dissipation of mean square density fluctuations. Using a gradient diffusion model and assuming constant eddy viscosity and diffusivities, Townsend showed that several equilibrium regimes are possible, where the production and the dissipation terms approximately balance. These assumptions led to a critical gradient Richardson number of approximately 0.25 , above which no equilibrium regimes are possible. At this point the region comprising the interface is essentially laminar. Turner also gives an inviscid analysis, using linearized stability theory, of a vortex sheet between two layers of un-mixed fluids. In this analysis the primary instability mechanism is the Kelvin-Helmholtz instability. Results indicate that the layer is stable for all gradient Richardson numbers greater than 0.25 [33].

Kranenburg [21] used a gradient diffusion model where the eddy viscosity and diffusivity were assumed to be functions of the gradient Richardson number to study horizontally homogeneous, density stratified shear flow. The eddy viscosity and diffusivity in this model were based on a set of transport equations that took effects of the finite adjustment time of these exchange coefficients into account. The transient development of the shear layer was studied, with the steady state being the desired outcome. Kranenburg found that, under uniform shear, an initial linear buoyancy distribution will develop a stepwise structure. Here the stepwise structure eventually forms two stratified layers that are separated by a region of sharp gradient in buoyancy and velocity, similar to that reported in Ruddick [26] and Moore and Long [23]. A stable linear distribution over part of the solution was also observed for various parameters in the model. 
Holt, Koseff, and Ferziger [15] performed direct numerical simulations of the transient development of a homogeneous stably stratified shear flow. The main objectives of their study were aimed at understanding the influence of the Richardson number, the Prandtl number, and the initial potential energy on the globally averaged statistics. Coherent structures were also investigated as functions of the Richardson number. Their study observed a critical Richardson number at which the mixing regime changes and the amount of anisotropy in the turbulent fluctuations is at a maximum.

Jacobitz, Sarkar and Van Atta [19] performed direct numerical simulations of turbulence evolution in a uniformly sheared, stably stratified flow. For these simulations, spectral methods were used for the spatial discretisation and a third order Runge-Kutta scheme was used for the temporal advancement. The turbulence evolution was found to be highly dependent on the gradient Richardson number, the initial value of the Reynolds number based on the Taylor micro scale, and the initial ratio of production to dissipation of turbulent kinetic energy. Typical initial conditions for these simulations consisted of a mean linear distribution for the shear and the buoyancy. Results from these simulations indicated a critical gradient Richardson number, above which the turbulence will follow an exponential decay and below which the turbulence will grow exponentially. This critical gradient Richardson number was found to be in the range $0.04<\mathrm{Ri}_{\mathrm{G}}<0.17$, and was found to depend strongly on the Reynolds number and the initial ratio of production to dissipation of turbulent kinetic energy. 
In the recent computational studies that have been performed, the majority of work seems to have been either simplified analysis of the governing equations or in depth studies using direct numerical simulations. While direct numerical simulations (DNS) can be very accurate and can give large amounts of information concerning the flow, DNS is very demanding on computational resources and is presently suitable only for relatively simple flows. It is therefore seen as beneficial to investigate the application of models that combine some amount of applicability to real world engineering problems on one hand, while still providing some level of complexity in their treatment of the multiphase flow and turbulent flow on the other hand. The current investigation follows this approach. 


\subsection{MULTIPHASE MODELS}

\subsection{Introduction to Multiphase Modeling}

Continuum multiphase models can be categorized into either mixture models or multifluid models [14]. True multiphase modeling involves the consideration of each phase as a distinct fluid, which leads to multi-fluid models. Here, the primary conservation equations of mass, momentum and energy are written individually for each phase, and the phases interact via inter-phase transfer terms. Primary applications for multi-fluid models involve cases where the general flow pattern of the two phases is not known, and the flow is not amenable to simplifying assumptions. These models are somewhat complicated because the introduction of numerous momentum equations in the formulation (i.e. one set of momentum equations per phase) presents mathematical difficulties and because proper specification of the inter-phase transfer terms is not always easy. Computationally, these models are expensive and difficult to solve. For a three dimensional, transient problem with a turbulence model, as many as 12-14 transport equations must be solved at each time step for a two-phase flow.

Another class of models, described as mixture models, are formulated by considering the multiphase mixture as a single fictitious fluid. These models are usually the result of first considering the equations given by a multi-fluid model and then making simplifying assumptions about the flow in question. The basic concept is to consider the two-phase mixture as a whole, rather than as two separate phases. These models make use of a set of mixture variables for the velocity, density, and viscosity as some combination of the 
unmixed phase values of these quantities. Here the crucial problem is the determination of the physical properties of the fictitious fluid, and the definitions of the mixture variables.

The multiphase models that were used for this study were mixture models, where only one set of momentum equations was solved for the given two-phase (two-fluid) flow. More specifically, two different models were used. One model was used for the simulations involving immiscible fluids, and the other model was used for the simulations involving miscible fluids. Both of these models are, to some extent, simplified variations of the drift flux model as given by Ishii [16]. Basically, the present study adopts the definitions for the mixture quantities and the form of the individual phase mass and momentum equations as given by Ishii [16]. From these definitions and equations a model is developed for turbulent flows where two liquids mix and the relative velocity between the liquids is non-zero as is typical for buoyant, immiscible flows. This model is described as being a single fluid, scalar transport (SFST) model in Section 3.2, and will be referred to as SFST2. For cases where the relative velocity between the phases is negligible, SFST2 can be further simplified to give a model that is typically used for miscible fluids. This model is referred to as SFST1 and is described in detail in Section 3.3.

Three of the main assumptions that are used in formulating the SFST models are that the individual phases are incompressible, that the flow is isothermal, and that the density difference between the phases is small compared to the density of either phase. These 
assumptions allow certain terms to be neglected in the form of the model equations for turbulent flows. Another important modeling assumption involves using a gradient diffusion model for the average turbulent stresses in the momentum equations. Each of the SFST models is described in the following sections, and a full derivation of the model equations is included in Appendix A. The equations in the SFST models that are presented in the following sections are written in terms of time averaged variables.

\subsection{SFST Model for Flows Involving Immiscible Fluids}

As with any mixture model, the basic concept is to consider the two-phase mixture as a whole, rather than as two separate phases. Following Ishii [16], the mixture variables are defined for SFST2 as the center of mass velocity

$$
u_{\mathrm{m}}=\frac{\left(\mathrm{u}_{\alpha} \mathrm{r}_{\alpha} \rho_{\alpha}+\mathrm{u}_{\beta} \mathrm{r}_{\beta} \rho_{\beta}\right)}{\rho_{\mathrm{m}}}
$$

mixture density

$$
\rho_{\mathrm{m}}=\rho_{\alpha} r_{\alpha}+\rho_{\beta} r_{\beta}
$$

and mixture viscosity

$$
\mu_{\mathrm{m}}=\mu_{\alpha} \mathrm{r}_{\alpha}+\mu_{\beta} \mathrm{r}_{\beta}
$$

where the unmixed phase quantities are denoted by the subscripts $\alpha$ and $\beta$ (i.e. $u_{\alpha}$ is the velocity of phase alpha) and $\mathrm{r}$ is the phase volume fraction. This model expresses the conservation of mass and momentum for the two-phase mixture by summing the individual phase equations to form one set of equations for the mixture mass and mixture momentum. In this manipulation, the inter-phase transfer terms, as would be found in a 
two-fluid model, cancel in the addition. The resulting equations are then manipulated to a form that is similar to the single phase Navier-Stokes equations where the variables of interest are the mixture variables. For the present model this derivation is presented in Appendix A.2 for the mixture mass equation and in Appendix A.5 for the mixture momentum equations.

Since the flow field is turbulent the model equations are modified to account for turbulent stresses and turbulent diffusion. In the present study the additional terms that appear in the equations because of the turbulence are modeled using an eddy viscosity model for the turbulent stresses and an eddy diffusivity model for the turbulent diffusion.

The mixture density fluctuations in the multiphase equations do present some complications. Though these fluctuations in the density are recognized to exist, for cases where the density of either phase is large compared to the density difference, the density fluctuations may be neglected in the continuity and momentum equations. A detailed proof of this is given in Appendix A.2. Fortunately for the cases under consideration in this study, the assumption about the density difference being small is valid.

Conservation of mass for the SFST models is expressed by the mixture continuity equation, which is given by

$$
\frac{\partial \rho_{m}}{\partial t}+\frac{\partial \rho_{m} u_{m, i}}{\partial x_{i}}=0
$$


Eq. (3.2.4) is derived from the individual phase continuity equations as is shown in Appendix A.2. The main assumption in obtaining Eq. (3.2.4) is that the turbulent correlations between velocity and density in the mixture density may be neglected. It should be noted that these correlations would not appear if density weighted averaging (i.e. Favre averaging) was used.

As mentioned earlier the individual phase momentum equations are used as the basis for the mixture momentum equation in the SFST models. Basically, the individual phase equations are added and the resulting equation is put into a form that is similar to the single-phase Navier-Stokes equations. Also, the turbulent stresses are accounted for by using an eddy viscosity model which will be discussed in greater detail in Chapter 4. For SFST2, the resulting momentum equations for a turbulent flow are given by

$$
\begin{gathered}
\frac{\partial}{\partial t}\left[\rho_{m} u_{m, i}\right]+\frac{\partial}{\partial x_{j}}\left[\rho_{m} u_{m, i} u_{m, j}\right]=-\frac{\partial}{\partial x_{j}}\left[\frac{r_{\alpha} \rho_{\alpha} r_{\beta} \rho_{\beta}}{\rho_{m}}\left(u_{s, i} u_{s, j}\right)\right] \\
-\frac{\partial P}{\partial x_{i}}+\frac{\partial}{\partial x_{j}}\left(\left(\mu_{m}+\mu_{T}\right)\left(\frac{\partial u_{m, i}}{\partial x_{j}}+\frac{\partial u_{m, j}}{\partial x_{i}}\right)\right)+\rho_{m} g_{i}
\end{gathered}
$$

where the phases are denoted by $\alpha$ and $\beta$ and the subscripts $i$ and $j$ indicate summation when repeated. The full derivation of Eq. (3.2.5) is given in Appendix A.5. The slip or relative velocity in Eq. (3.2.5) is given by

$$
\mathrm{u}_{\mathrm{S}, \mathrm{i}}=\left(\mathrm{u}_{\alpha, \mathrm{i}}-\mathrm{u}_{\beta, \mathrm{i}}\right)
$$

In the mixture momentum equation, an additional flux due to the relative motion or slip between the phases appears as the first term on the right side of the equation. This extra term is sometimes referred to as the drift flux term and originates from using the mixture 
velocity definition in the convective terms as is shown in Appendix A.5. Essentially this extra term accounts for the macroscopic flux of momentum due to the relative motion between the phases, and is written on the right hand side of the equation as an additional fictitious stress. This is somewhat analogous to the microscopic transport of momentum due to the molecular transport that produces viscosity.

Since these manipulations and definitions are little more than algebraic maneuvers, no immediate advantage is gained by adding the individual conservation equations. However, the primary assumption of a mixture model is that the dynamics of the two phases can be expressed by the preceding mixture momentum equation and some algebraic equation for the slip velocity between the phases. In a general sense, the model assumes that some form of the slip velocity relation given by

$$
\mathrm{u}_{\mathrm{s}}=f\left(\mathrm{r}_{\alpha}, \rho_{\mathrm{m}}, \mathrm{u}_{\mathrm{m}}, \mathrm{g}, \text { etc. }\right)
$$

can be described empirically or by simple algebraic models. This assumption is justified if the motion of the two phases is strongly coupled. Since the freedom of using one of the single phase momentum equations is still available, it can be used to aid in determining the slip velocity equation. Making simplifying assumptions, a kinematic constitutive equation for the relative or slip velocity between the phases can be determined. A relatively simple model is to assume that the slip velocity is a function of the terminal velocity of an average size droplet. For flows in which more extensive relationships are needed, experiments can be used to aid in determining equations for the slip velocity. These assumptions reduce the two individual phase momentum equations into a single momentum equation with one extra term that is a function of the slip velocity. 
Typically the volume fractions are solved from one of the individual phase continuity equations and from the algebraic constraint that the volume fractions must sum to one (i.e. $r_{\alpha}+r_{\beta}=1$ ). It should be noted that in a turbulent flow, the turbulent fluctuations in the volume fraction must be maintained in the formulation of the volume fraction equation. Hence to account for turbulent diffusion of the volume fraction, the fluctuating correlations are modeled using an eddy diffusivity model, and the time averaged form of the modeled volume fraction equation in SFST2 is given by

$$
\frac{\partial\left(\rho_{\alpha} r_{\alpha}\right)}{\partial t}+\frac{\partial}{\partial x_{i}}\left(\rho_{\alpha} r_{\alpha} u_{m, i}\right)=-\frac{\partial}{\partial x_{i}}\left[\frac{\rho_{\alpha} r_{\alpha} r_{\beta} \rho_{\beta}}{\rho_{m}} u_{S, i}\right]+\frac{\partial}{\partial x_{i}}\left[\frac{\rho_{\alpha}}{\rho_{m}} \Gamma_{T}\left(\frac{\partial r_{\alpha}}{\partial x_{i}}\right)\right]
$$

as is shown in Appendix A.4. Here the slip velocity is shown to have the influence of an additional convective term. Methods for modeling the eddy diffusivity, given by $\Gamma_{\mathrm{T}}$, are given in Section 4.0.

One further modification can be made to Eq. (3.2.8) that provides an efficient means of implementing SFST2 into any standard single fluid code. By the nature of the transport process, the volume fraction is conveyed on a unit volume basis; however, the typical scalar transport equation found in most codes is formulated by considering a scalar that is formulated on a unit mass basis. For instance in CFX 4.1, $\rho_{\mathrm{m}}$ appears in the convective and diffusive terms in the scalar transport equations that are available to the user. To avoid solving Eq. (3.2.8) explicitly, it would be advantageous to write Eq. (3.2.8) in the typical form of a scalar transport equation. This can be accomplished by using the fact that the divergence of the mixture velocity is zero and by making a minor approximation 
in the diffusion term. This derivation is given in Appendix A.4 and shows that Eq. (3.2.8) can be written in the form of a scalar transport equation as

$$
\frac{\partial\left(\rho_{\mathrm{m}} \mathrm{r}_{\alpha}\right)}{\partial \mathrm{t}}+\frac{\partial}{\partial \mathrm{x}_{\mathrm{i}}}\left(\rho_{\mathrm{m}} \mathrm{r}_{\alpha} \mathrm{u}_{\mathrm{m}, \mathrm{i}}\right)=-\rho_{\mathrm{m}} \frac{\partial}{\partial \mathrm{x}_{\mathrm{i}}}\left[\frac{\mathrm{r}_{\alpha} \mathrm{r}_{\beta} \rho_{\beta}}{\rho_{\mathrm{m}}} \mathrm{u}_{\mathrm{S}, \mathrm{i}}\right]+\frac{\partial}{\partial \mathrm{x}_{\mathrm{i}}}\left[\Gamma_{\mathrm{T}}\left(\frac{\partial \mathrm{r}_{\alpha}}{\partial \mathrm{x}_{\mathrm{i}}}\right)\right]
$$

where the mixture density has replaced the density of phase $\alpha$ in the convective terms. Eq. (3.2.9) is equivalent to a generic scalar transport equation with one additional source term that is a function of the slip velocity. Upon solving Eq. (3.2.9) the volume fraction of the second phase is easily determined from the constraint that the volume fractions must sum to one. As an added note, the divergence of the mixture velocity is zero if the divergence of each of the unmixed phase densities, $\rho_{\alpha}$ and $\rho_{\beta}$, are zero; this is shown in Appendix A.3.

In summary, Eqs. (3.2.4), (3.2.5), (3.2.7), and (3.2.9) represent four independent equations that can be used to solve for the mixture velocity, pressure, phase volume fractions, and relative velocity between the phases. This model maintains the provision of reducing to a set of single phase equations having strongly varying properties and some additional terms to account for the relative velocity, which may be easily added in any single phase code. Hence the name single fluid, scalar transport model, is based on the model's close ties to its corresponding single phase equations. It should be noted that SFST2 does provide for the expected density variation in the continuity and momentum equations and has provisions for relative velocity effects between the phases. These capabilities are important features of the model, making it attractive for any extensive computational effort at predicting immiscible multiphase flows. 


\subsection{SFST Model for Flows Involving Miscible Fluids}

As the simplest multiphase model, SFST1, the SFST model that was used for the simulations involving miscible flows, assumes that the relative velocity between the phases is zero. Hence only one velocity field prevails. This model is generally accepted to be valid for cases where the inter-phase drag equalizes quickly when two fluids mix and for cases involving free surface flows where the phases are only slightly mixed at the interface. This model is not valid for cases where either of the phases will attain a significant non-zero slip velocity. With the restriction on the velocity of the individual phases, the slip velocity is zero and the mixture momentum equations given by Eq. (3.2.5) reduce to a set of single fluid equations with varying properties.

Conservation of mass for SFST1 is expressed by the same time averaged equation that was used for SFST2, given by

$$
\frac{\partial \rho_{m}}{\partial t}+\frac{\partial}{\partial x_{i}}\left(\rho_{m} u_{m, i}\right)=0
$$

With the assumption that the individual phase velocities are equal, the momentum equation in SFST1 is given by

$$
\frac{\partial\left(\rho_{m} u_{m, i}\right)}{\partial t}+\frac{\partial}{\partial x_{j}}\left(\rho_{m} u_{m, i} u_{m, j}\right)=-\frac{\partial P}{\partial x_{i}}+\frac{\partial}{\partial x_{j}}\left[\left(\mu_{m}+\mu_{T}\right)\left(\frac{\partial u_{m, i}}{\partial x_{j}}+\frac{\partial u_{m, j}}{\partial x_{i}}\right)\right]+\rho_{m} g_{i}
$$

where $\mu_{\mathrm{T}}$ is the eddy viscosity. As in SFST2, the density and viscosity are given by Eq. (3.2.2) and Eq. (3.2.3) respectively. 
The modeled form of the volume fraction equation in SFST1 is given by

$$
\frac{\partial\left(\mathrm{r}_{\alpha} \rho_{\alpha}\right)}{\partial \mathrm{t}}+\frac{\partial}{\partial \mathrm{x}_{\mathrm{i}}}\left(\mathrm{r}_{\alpha} \rho_{\alpha} \mathrm{u}_{\mathrm{m}, \mathrm{i}}\right)=\frac{\partial}{\partial \mathrm{x}_{\mathrm{i}}}\left(\frac{\rho_{\alpha}}{\rho_{\mathrm{m}}} \Gamma_{\mathrm{T}} \frac{\partial \mathrm{r}_{\alpha}}{\partial \mathrm{x}_{\mathrm{i}}}\right)
$$

where $\Gamma_{\mathrm{T}}$ is the eddy diffusivity. Eq. (3.3.3) can be written in the form of a scalar transport equation as

$$
\frac{\partial\left(r_{\alpha} \rho_{m}\right)}{\partial t}+\frac{\partial}{\partial x_{i}}\left(r_{\alpha} \rho_{m} u_{m, i}\right)=\frac{\partial}{\partial x_{i}}\left[\Gamma_{T}\left(\frac{\partial r_{\alpha}}{\partial x_{i}}\right)\right]
$$

This is accomplished by making use of the mixture continuity equation, and a detailed rationale of this derivation is given in Appendix A.4. This is one of the most attractive features of this model; under the previously mentioned assumptions it reduces to a set of single phase equations having variable properties and a single scalar transport equation. The solution of such a system may be accomplished easily with any single phase code that allows the user to solve a scalar transport equation and specify functions for the density and viscosity.

In the literature concerning multiphase flows, the form of the mathematical model obtained by assuming that the phase velocity fields are the same, is referred to as the homogeneous multiphase model as is given in Hetsroni [14]. The term homogeneous typically describes two miscible fluids. Its main application is for cases where two fluids attain equal velocities quickly as they mix and basically form a new fluid with a variable density. Salt water and fresh water mixing or hot and cold water mixing are good examples. Immiscible fluids such as diesel fuel and water would not be well suited for this model as they typically tend toward some relative velocity under gravity. For the 
present study the title homogeneous is not adopted to avoid confusion with the default homogeneous multiphase model in CFX 4.1, which was not used for the simulations. The simulations were performed using the single phase momentum equations and an additional scalar transport equation for the light phase volume fraction, where the density and viscosity changed as functions of the volume fractions. Hence the model is referred to as SFST1. The default CFX model was not used because the SFST models were found to exhibit faster numerical convergence and were less likely to diverge when applied to complicated geometries. 


\subsection{MULTIPHASE TURBULENCE MODELS}

\subsection{Introduction to Turbulence Modeling for Multiphase Flows}

While turbulence modeling has made significant progress in providing models that give reasonable engineering predictions for a wide class of flows, their is no "industrial standard" model for turbulent multiphase flows [6]. The general approach has therefore been to use the single-phase form of a turbulence model and assume the model is sufficient to account for the desired features of the turbulence in a multiphase flow. This is equivalent to assuming that the turbulence of each phase is approximately the same at each point in the flow field. Additional transport terms are then usually added to the turbulence model to account for any significant effects that the two-phase mixture might have on the turbulence, such as buoyant production or dissipation of turbulent kinetic energy.

Two-equation models have been the most popular turbulence models for a wide range of engineering analysis and research. These models provide independent transport equations for both the turbulence length scale, or some equivalent parameter, and the turbulent kinetic energy. This section will focus primarily on the use of the $k$ - $\varepsilon$ model, where $k$ is the turbulent kinetic energy and $\varepsilon$ is the rate of dissipation of $k$, and its application to buoyancy dominated, multiphase flows. The first part of this section will give a brief introduction to the single phase form of the $k-\varepsilon$ model, followed by a description of the modified form of the $k-\varepsilon$ model for buoyant flows. 


\subsection{Standard k- $\varepsilon$ Model}

The $k-\varepsilon$ model attempts to provide equations for a characteristic velocity scale, denoted by $k$, based on the kinetic energy of the turbulent fluctuations and for a characteristic length scale via the dissipation rate of $k$, denoted by $\varepsilon$. This section will give a brief overview concerning the assumptions made in formulating the $k-\varepsilon$ model, the form of the transport equations, and the auxiliary equations for the model.

A major assumption of the $k-\varepsilon$ model is that the root mean square turbulent fluctuations, $\left(\sqrt{\mathrm{u}^{\prime 2}}, \sqrt{\mathrm{v}^{\prime 2}}, \sqrt{\mathrm{w}^{\prime 2}}\right)$, are locally isotropic or equal. While this is true of the smaller eddies at high Reynolds numbers, the large eddies are in a state of steady anisotropy due to the strain rate of the mean flow, though the root mean square fluctuations are almost always of the same order of magnitude. Implicit in this assumption is the implication that the normal Reynolds stresses are also isotropic. For flows where there are appreciable density gradients, the loss of turbulent kinetic energy to buoyancy affects $v^{\prime}$ directly. Thus the buoyancy, while possibly only dissipating a small amount of the turbulence, makes its contribution by fundamentally changing any isotropy in the turbulent fluctuations by decreasing v' [33].

Another major assumption of the $k-\varepsilon$ model is that the production and dissipation terms, given in the $k$-equation, are approximately equal locally. This is known as the local equilibrium assumption. To allow the Reynolds stresses to be calculated using local scales, the $k-\varepsilon$ model assumes that production approximately equals dissipation in the $k$ - 
equation. In general, the $k-\varepsilon$ model uses the Boussinesq eddy-viscosity concept where the turbulent stresses are given by

$$
\tau_{\mathrm{ij}}=-\rho \overline{u_{\mathrm{i}}^{\prime} \mathrm{u}_{\mathrm{j}}^{\prime}}=\mu_{\mathrm{T}}\left(\frac{\partial \mathrm{u}_{\mathrm{i}}}{\partial \mathrm{x}_{\mathrm{j}}}+\frac{\partial \mathrm{u}_{\mathrm{j}}}{\partial \mathrm{x}_{\mathrm{i}}}\right)-\frac{2}{3} k \delta_{\mathrm{ij}}
$$

where $\mu_{\mathrm{T}}$ is the isotropic eddy viscosity. The second quantity on the right hand side involving $k$ has been included to ensure the correct normal Reynolds stress in the absence of any strain rate. This term involving $k$ is usually absorbed into the pressure term in the momentum equation, allowing the viscosity in the momentum equation to be written as the sum of the molecular and eddy viscosity. Since the turbulent and mean scales are proportional, the eddy viscosity can be estimated based on dimensional and physical reasoning by using either the turbulent or mean scales. This implies that

$$
\mu_{\mathrm{T}} \propto \frac{\rho k^{2}}{\varepsilon}
$$

for the $k$ - $\varepsilon$ model. In a manner similar to that used to obtain the eddy viscosity, the eddy diffusivity for the volume fraction is assumed to be given using a turbulent Prandtl number, $\sigma_{\mathrm{r}}$, as

$$
\Gamma_{\mathrm{T}}=\frac{\mu_{\mathrm{T}}}{\sigma_{\mathrm{r}}} \propto \frac{\rho k^{2}}{\sigma_{\mathrm{r}} \varepsilon}
$$

Note that as a result, both $\mu_{\mathrm{T}}$ and $\Gamma_{\mathrm{T}}$ are isotropic locally, i.e. the diffusivity does not change with direction. If production does not balance dissipation locally then the ratio of the Reynolds stress to the mean strain rate is not a local constant and $\mu_{\mathrm{T}}$ and $\Gamma_{\mathrm{T}}$ will be functions of both turbulent and means scales. Another way to understand this assumption of local equilibrium is to realize that transport effects, while included for the turbulent 
scales, are neglected for the turbulent Reynolds stresses. Local scales are then used in estimating the Reynolds stresses. Otherwise the Reynolds stresses would depend on the local scales plus some combination of scales from upstream and possibly from downstream. If the flow is slowly varying then upstream scales are about the same as local scales of turbulence and the equilibrium assumption will be valid. Also, if the turbulence is evolving at a sufficiently rapid rate, such that the effects of past events do not dominate the dynamics, the estimates based on local scales will be appropriate.

Since the exact equation for $k$ and $\varepsilon$ can be found in many texts, the modeled forms used in the present study will be presented here along with the auxiliary equations used for such quantities as the eddy viscosity. The modeled $k$-equation is given by

$$
\frac{\partial \rho k}{\partial \mathrm{t}}+\frac{\partial \rho \mathrm{u}_{\mathrm{j}} k}{\partial \mathrm{x}_{\mathrm{j}}}=\tau_{\mathrm{ij}} \frac{\partial \mathrm{u}_{\mathrm{i}}}{\partial \mathrm{x}_{\mathrm{j}}}-\rho \varepsilon+\frac{\partial}{\partial \mathrm{x}_{\mathrm{j}}}\left(\left(\mu+\frac{\mu_{\mathrm{T}}}{\sigma_{\mathrm{k}}}\right) \frac{\partial k}{\partial \mathrm{x}_{\mathrm{j}}}\right)
$$

where the terms on the right hand side represent production, dissipation, and viscous and turbulent diffusion. The modeled $\varepsilon$-equation is given by

$$
\frac{\partial \rho \varepsilon}{\partial \mathrm{t}}+\frac{\partial \rho \mathrm{u}_{\mathrm{j}} \varepsilon}{\partial \mathrm{x}_{\mathrm{j}}}=\mathrm{C}_{\varepsilon 1} \frac{\varepsilon}{k} \tau_{\mathrm{ij}} \frac{\partial \mathrm{u}_{\mathrm{i}}}{\partial \mathrm{x}_{\mathrm{j}}}-\mathrm{C}_{\varepsilon 2} \rho \frac{\varepsilon^{2}}{k}+\frac{\partial}{\partial \mathrm{x}_{\mathrm{j}}}\left(\left(\mu+\frac{\mu_{\mathrm{T}}}{\sigma_{\varepsilon}}\right) \frac{\partial \varepsilon}{\partial \mathrm{x}_{\mathrm{j}}}\right)
$$

where the terms on the right hand side represent production of dissipation, dissipation of dissipation, and diffusion of dissipation. The turbulence model based on the equation for the rate of dissipation of $k$, is based on the exact equation for $\varepsilon$. Since the closure approximations used in modeling the exact equation for $\varepsilon$ are based primarily on largeeddy scales, it is implied that the modeled equation is actually more of an empirical equation representing the transfer of energy from the large eddies to the smaller eddies. 


\subsection{Modified k- $\varepsilon$ Model for Buoyant Flows}

While most turbulence is generated as a function of shear, in flows with appreciable density gradients the turbulent eddies may receive or lose energy due to the effects of buoyancy. In such flows, the mean momentum equation can be written as

$$
\frac{\partial \rho u_{i}}{\partial t}+\frac{\partial \rho u_{j} u_{i}}{\partial x_{j}}=-\frac{\partial p}{\partial x_{i}}+\frac{\partial}{\partial x_{j}}\left(\mu \overline{S_{i j}}\right)-\frac{\partial}{\partial x_{j}}\left(\overline{\rho u_{i}{ }^{\prime} u_{j}{ }^{\prime}}\right)+\left(\rho+\rho^{\prime}\right) g_{i}
$$

where the density fluctuations have been ignored in the inertial terms and maintained in the buoyancy or gravitational terms. Using the mean momentum equation, it can be shown that the exact specific turbulent kinetic energy equation is given by

$$
\begin{aligned}
& \frac{\partial \rho k}{\partial \mathrm{t}}+\frac{\partial \rho \mathrm{u}_{\mathrm{j}} k}{\partial \mathrm{x}_{\mathrm{j}}}=\tau_{\mathrm{ij}} \frac{\partial \mathrm{u}_{\mathrm{i}}}{\partial \mathrm{x}_{\mathrm{j}}}-\mu \overline{\frac{\mathrm{u}_{\mathrm{i}}}{\partial \mathrm{x}_{\mathrm{k}}} \frac{\mathrm{u}_{\mathrm{i}}^{\prime}}{\partial \mathrm{x}_{\mathrm{k}}}}+\overline{\mathrm{u}_{\mathrm{i}}{ }^{\prime} \rho^{\prime} \mathrm{g}} \\
& +\frac{\partial}{\partial \mathrm{x}_{\mathrm{j}}}\left(\mu \frac{\partial k}{\partial \mathrm{x}_{\mathrm{j}}}-\frac{1}{2} \rho \overline{\mathrm{u}_{\mathrm{i}}{ }^{\prime} \mathrm{u}_{\mathrm{i}}{ }^{\prime} \mathrm{u}_{\mathrm{j}}{ }^{\prime}}-\overline{\mathrm{p}^{\prime} \mathrm{u}_{\mathrm{j}}{ }^{\prime}}\right)
\end{aligned}
$$

where again, the density fluctuations in the inertial terms have been neglected and the sign of the gravity vector has not been taken into account. Each of the terms in Eq. (4.3.2) are accounted for in the original modeled $k$-equation, except for the extra term represented by

$$
\mathrm{G}_{\mathrm{k}}=\overline{\mathrm{u}_{\mathrm{i}}{ }^{\prime} \rho^{\prime} \mathrm{g}}
$$

which is referred to as buoyant production and represents the rate of work against buoyancy forces by the turbulent motion. This is essentially a transfer of either potential energy to turbulent kinetic energy as would be the case in an unstably stratified fluid, or a transfer of turbulent kinetic energy to potential energy as would be the case in the mixing of a heavy fluid with a lighter fluid against the action of gravity. 
The buoyancy production term is usually modeled as

$$
G_{k}=-\left|g_{i}\right| \overline{u_{i} \rho^{\prime}}=\left|g_{i}\right| \frac{\mu_{T}}{\rho \sigma_{\rho}} \frac{\partial \rho}{\partial x_{i}}
$$

in a coordinate system where gravity acts in the negative coordinate direction [25]. Here $\sigma_{\rho}$ is a kind of turbulent Prandtl number that is usually taken equal to 1.0. The density derivative is used since a negative density gradient usually corresponds to a stably stratified flow and the term acts as a sink on turbulent kinetic energy. Here the correlation between the fluctuating vertical velocity component and fluctuating density tends to be positive. For positive density gradients an unstable stratification usually exists and the term acts as a source for $k$; this corresponds to the negative correlation between the fluctuating vertical velocity component and fluctuating density.

This simple gradient-diffusion model for the buoyancy flux or production term, does have experimental support for nearly homogeneous cases as is given in Narimousa [24]. The epsilon equation is typically modified by adding the source term given by

$$
\mathrm{G}_{\varepsilon}=\frac{\mathrm{C}_{\varepsilon 1} \varepsilon}{k} \mathrm{C}_{\varepsilon 3} \max \left(\mathrm{G}_{\mathrm{k}}, 0\right)
$$

where $C_{\varepsilon 3}$ is a constant [25]. This term increases $\varepsilon$ for unstable stratification and gives no change in $\varepsilon$ for stable stratification. 
Having defined the model equations, the $k$ - $\varepsilon$ turbulence model for buoyant flows that was used in all of the numerical simulations is given by

the $k$-equation

$$
\frac{\partial \rho k}{\partial \mathrm{t}}+\frac{\partial \rho \mathrm{u}_{\mathrm{j}} k}{\partial \mathrm{x}_{\mathrm{j}}}=\tau_{\mathrm{ij}} \frac{\partial \mathrm{u}_{\mathrm{i}}}{\partial \mathrm{x}_{\mathrm{j}}}-\rho \varepsilon+\frac{\partial}{\partial \mathrm{x}_{\mathrm{j}}}\left(\left(\mu+\frac{\mu_{\mathrm{T}}}{\sigma_{\mathrm{k}}}\right) \frac{\partial k}{\partial \mathrm{x}_{\mathrm{j}}}\right)+|\mathrm{g}| \frac{\mu_{\mathrm{T}}}{\rho \sigma_{\rho}} \frac{\partial \rho}{\partial \mathrm{y}}
$$

the $\varepsilon$-equation

$$
\begin{gathered}
\frac{\partial \rho \varepsilon}{\partial \mathrm{t}}+\frac{\partial \rho \mathrm{u}_{\mathrm{j}} \varepsilon}{\partial \mathrm{x}_{\mathrm{j}}}=\mathrm{C}_{\varepsilon 1}\left(\frac{\varepsilon}{k} \tau_{\mathrm{ij}} \frac{\partial \mathrm{u}_{\mathrm{i}}}{\partial \mathrm{x}_{\mathrm{j}}}+\frac{\varepsilon}{k} \mathrm{C}_{\varepsilon 3} \max \left(\mathrm{G}_{\mathrm{k}}, 0\right)\right)+ \\
-\mathrm{C}_{\varepsilon 2} \rho \frac{\varepsilon^{2}}{k}+\frac{\partial}{\partial \mathrm{x}_{\mathrm{j}}}\left(\left(\mu+\frac{\mu_{\mathrm{T}}}{\sigma_{\varepsilon}}\right) \frac{\partial \varepsilon}{\partial \mathrm{x}_{\mathrm{j}}}\right)
\end{gathered}
$$

where

$$
\begin{gathered}
\mu_{T}=\frac{\rho C_{\mu} k^{2}}{\varepsilon} \\
\Gamma_{T}=\frac{\mu_{T}}{\sigma_{r}} \\
\tau_{i j}=-\rho \overline{u_{i}^{\prime} u_{j}^{\prime}}=\mu_{T}\left(\frac{\partial u_{i}}{\partial x_{j}}+\frac{\partial u_{j}}{\partial x_{i}}\right)-\frac{2}{3} k \delta_{i j}
\end{gathered}
$$

with the closure coefficients given by
$\mathrm{C}_{\varepsilon 1}=1.44, \quad \mathrm{C}_{\varepsilon 2}=1.92$
$\mathrm{C}_{\varepsilon 3}=1.0$,
$\mathrm{C}_{\mu}=0.09$,
$\sigma_{\mathrm{k}}=1.0$,
$\sigma_{\varepsilon}=1.3$,
$\sigma_{\mathrm{r}}=1.0, \quad \sigma_{\rho}=1.0$ 


\subsection{SIMULATION OF STRATIFIED, SHEARING FLOW OF MISCIBLE FLUIDS}

Sullivan and List [28] performed experiments by measuring tracer dye concentrations in a homogeneous, density stratified, turbulent, shear flow. The flow scenario described in these experiments is shown schematically in Figure 5.1. Here $\mathrm{U}, \mathrm{h}$, and $\rho$ denote the free stream velocity, concentration boundary layer, and unmixed density for each layer, where the top and bottom layers are denoted by 1 and 0 respectively.

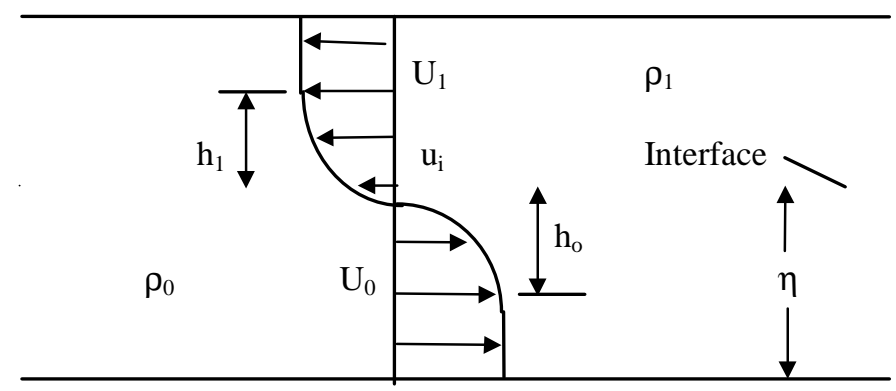

Figure 5.1 - Idealized shear flow for Sullivan and List experiments.

Four cases from Sullivan and List's experiments were simulated using the commercial code, CFX 4.1. Time averaged, steady state solutions were obtained using SFST1, and the $k$ - $\varepsilon$ turbulence model with the buoyancy source terms. The simulations were compared with all measured experimental quantities, including predicted Richardson numbers, trends in interfacial mixing, and trends in the velocity field. Of primary interest in these simulations was the ability to predict trends for the interfacial thickness, mixed fluid thickness, and characteristic Richardson numbers. 


\subsection{Experimental Conditions}

The experimental scenario consisted of a counter flow that was generated by two inlets at the opposite ends of a $5.0 \mathrm{~m}$ long by $20.0 \mathrm{~cm}$ high by $10.0 \mathrm{~cm}$ wide laboratory channel as is shown in Figure 5.2. The heavy fluid (aqueous saline solution) and the light fluid

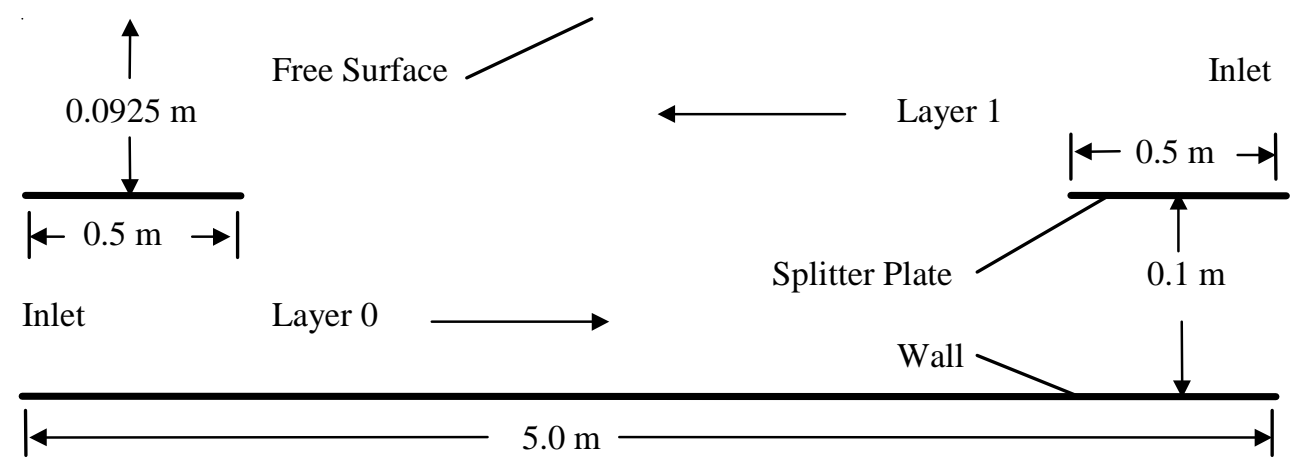

Figure 5.2 - Sullivan and List experimental setup.

(aqueous ethanol solution) were separated by splitter plates at the inlets. The lower wall was covered by a layer of small rocks, with an average roughness height of $0.75 \mathrm{~cm}$. In the experiments this was done to study the effects of the wall generated turbulence; however, this rough wall did little to influence the mixing [28]. This is consistent with what has been observed in several other experiments, essentially that most of the turbulence generated at a wall dissipates near the wall [24]. The major portion of the turbulence for this experiment was generated by the shear at the interface, and only a small amount diffused from the wall. The top of the flow was left open to the air. Figure 5.3 shows a schematic of the complete shear flow facility as given by Sullivan [27]. 


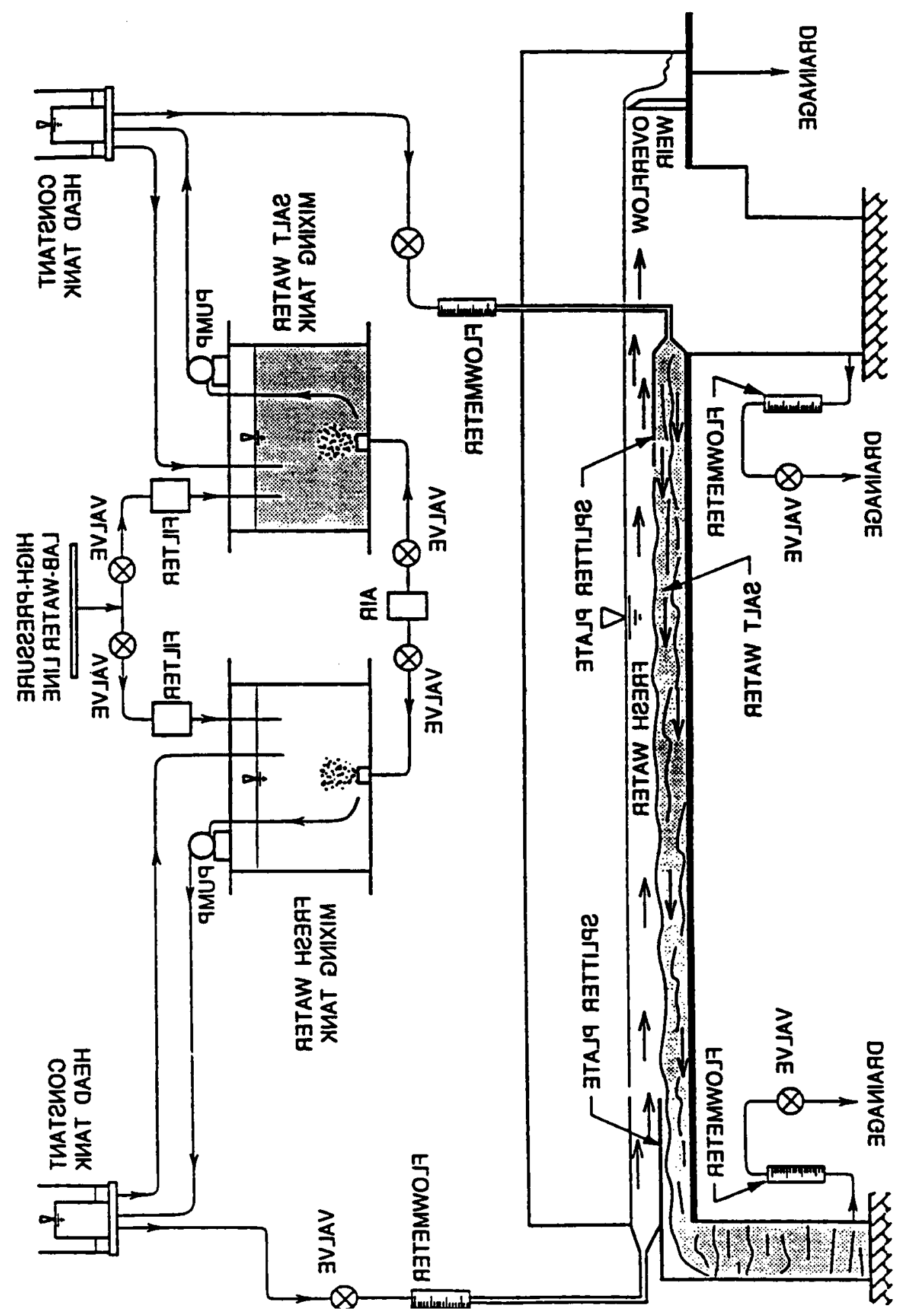

Figure 5.3 - Experimental facility for experiments; After Sullivan [28]. 
Typical inlet flow rates were approximately $40.0 \mathrm{~cm}^{3} / \mathrm{s}$ per unit width, corresponding to inlet velocities of approximately $4.0 \mathrm{~cm} / \mathrm{s}$ and inlet Reynolds numbers of approximately 4,000 based on the inlet height. The buoyancy ranged from 5.0 to $20.0 \mathrm{~cm} / \mathrm{s}^{2}$. These experiments were performed over a range of shear Richardson numbers varying from 0.1 to 1.0, which corresponded to a Kelvin-Helmholtz and a shear driven, wave breaking mixing regime. In these experiments the relative importance of turbulence and buoyancy scales in each layer can be expressed by the layer Richardson number, given here as

$$
\mathrm{Ri}_{1}=\frac{\mathrm{hb}}{\left(\mathrm{U}-\mathrm{u}_{\mathrm{i}}\right)^{2}}
$$

for the free stream velocities from each layer. Another parameter which was measured for these experiments was the shear Richardson number which is defined here by

$$
\mathrm{Ri}_{\mathrm{S}}=\frac{\mathrm{bh}_{\mathrm{s}}}{\left(\mathrm{U}_{0}-\mathrm{U}_{1}\right)^{2}}
$$

The other governing parameters are described mostly in terms of the concentration or volume fraction of the upper layer, denoted by $r_{\alpha}$. To determine the location of the interface, Sullivan (1992) defines an interfacial half width, $\delta_{0}$, as the vertical distance between the center of the interface and the point where the concentration equals either $0.9 \mathrm{r}_{\alpha}$ or $0.1 \mathrm{r}_{\alpha}$. With this definition

$$
\begin{aligned}
& \delta_{0}=\eta-y\left(r_{\alpha}=0.1\right) \\
& \delta_{1}=y\left(r_{\alpha}=0.9\right)-\eta
\end{aligned}
$$

and

$$
\delta=y\left(r_{\alpha}=0.9\right)-y\left(r_{\alpha}=0.1\right)
$$


for a situation where the volume fraction is equal to one for the unmixed upper layer, and zero for the unmixed lower layer. Based on these definitions Sullivan defines a mean interface position,$\eta$, which can be determined from

$$
\eta=\frac{\left(\delta_{0}+\delta_{1}\right)}{2}+y\left(r_{\alpha}=0.1\right)
$$

$\eta$ is also referred to as the concentration interface. The concentration boundary layers, $\mathrm{h}_{0}$ and $\mathrm{h}_{1}$, are defined as the distance between the mean interface position and the point where the probability of finding fluid different in concentration from the local mean is 0.01. Sullivan and List comment that physically this is a measure to which the scalar is transported by turbulent motion. It is difficult to define a quantity which would strictly match this definition involving a time averaged concentration. Hence, in the simulations, the concentration boundary layer was defined as the distance above or below the mean interface where the upper layer volume fraction was either 0.01 or 0.99 , giving

$$
\begin{aligned}
& \mathrm{h}_{0}=\eta-\mathrm{y}\left(\mathrm{r}_{\alpha}=0.01\right) \\
& \mathrm{h}_{1}=\mathrm{y}\left(\mathrm{r}_{\alpha}=0.99\right)-\eta
\end{aligned}
$$

For the present simulations, another parameter referred to as the mixed fluid thickness, which should be distinguished from the interfacial thickness, is defined as the sum of the concentration boundary layers and is given by

$$
\delta_{\mathrm{M}}=\mathrm{h}_{\mathrm{O}}+\mathrm{h}_{1}
$$

Essentially, $\delta_{\mathrm{M}}$ is the thickness of the entire layer over which mixing occurs. 
The free stream velocity for each layer was based on the exit and entrance flow rates and layer thickness. The lower layer thickness, $\mathrm{d}$, was defined in terms of the concentration interface, where $d$ equals $\eta$. With $Q_{1}$ denoting the flow rate per unit length of the top fluid into the domain and $\mathrm{Q}_{4}$ specifying the flow rate of the bottom fluid out of the domain, free stream velocities were defined as

$$
\begin{aligned}
& \mathrm{U}_{0}=\frac{\mathrm{Q}_{4}}{\mathrm{~d}} \\
& \mathrm{U}_{1}=\frac{\mathrm{Q}_{1}}{(\mathrm{H}-\mathrm{d})}
\end{aligned}
$$

In the experiments the mean interface velocity, $\mathrm{u}_{\mathrm{i}}$, was estimated by linear interpolation. In the present simulations $\mathrm{u}_{\mathrm{i}}$ was linearly interpolated based on the location of the mean interface position as calculated by the code. The maximum velocity gradient and maximum velocity gradient thickness were also calculated based on their definitions, for calculating the shear Richardson number. In addition to the Richardson numbers used to characterize the experiments, an additional Richardson number was used that provided a reasonable means for comparing the experiments and the simulations. An overall Richardson number, given by

$$
\mathrm{Ri}^{*}=\frac{\mathrm{Hb}}{\left(\Delta \mathrm{U}_{\text {Inlet }}\right)^{2}}
$$

was defined where $\mathrm{H}$ is the total height of the test apparatus and $\Delta \mathrm{U}$ is defined in terms of the inlet velocities of the two layers. Since this Richardson number does give a measure of shear to buoyancy and is the same for the experiments and the simulations it provided an independent variable that could be adjusted. 
The mixed layer Richardson number, which is a type of combined layer Richardson number, was also calculated from the experimental data and from the simulations. This Richardson number was referred to as the mixed layer Richardson and was defined by

$$
\mathrm{Ri}_{\mathrm{L}}=\frac{\delta_{\mathrm{M}} \mathrm{b}}{\left(\Delta \mathrm{U}_{\text {Inlet }}\right)^{2}}
$$

where again $\Delta \mathrm{U}$ is defined in terms of the inlet velocities of the two layers.

\subsection{Computational Details}

In light of the experimental scenario and parameters described above, a two-dimensional model was configured in CFX 4.1. Four cases as described by Sullivan and List [27] were simulated, corresponding to the experiments labeled 1, 10, 11, and 13. Governing parameters for these experiments are given in Table 5.1.

Table 5.1 - Experimental Flow Parameters

$\begin{array}{cccccccc}\text { Case } & \mathrm{b}\left(\mathrm{cm} / \mathrm{s}^{2}\right) & \mathrm{Q}_{0}\left(\mathrm{~cm}^{2} / \mathrm{s}\right) & \mathrm{Q}_{1}\left(\mathrm{~cm}^{2} / \mathrm{s}\right) & \mathrm{H}_{\mathrm{O}}(\mathrm{cm}) & \mathrm{H}_{1}(\mathrm{~cm}) & \mathrm{H}_{2}(\mathrm{~cm}) & \mathrm{H}_{3}(\mathrm{~cm}) \\ 1 & 18.81 & 36.82 & 38.97 & 10.00 & 9.25 & 10.00 & 9.25 \\ 10 & 12.77 & 38.74 & 39.75 & 10.00 & 9.25 & 10.00 & 9.25 \\ 11 & 7.48 & 38.74 & 26.42 & 10.00 & 9.25 & 10.00 & 9.25 \\ 13 & 18.95 & 38.74 & 39.75 & 10.00 & 9.25 & 10.00 & 9.25\end{array}$

To simulate the experimental setup, a six block geometry was used as is shown in Figure 5.4. Here the inlets and outlets are shown as they were modeled in CFX. The dimensions of this model followed those as were used in the experiments. 


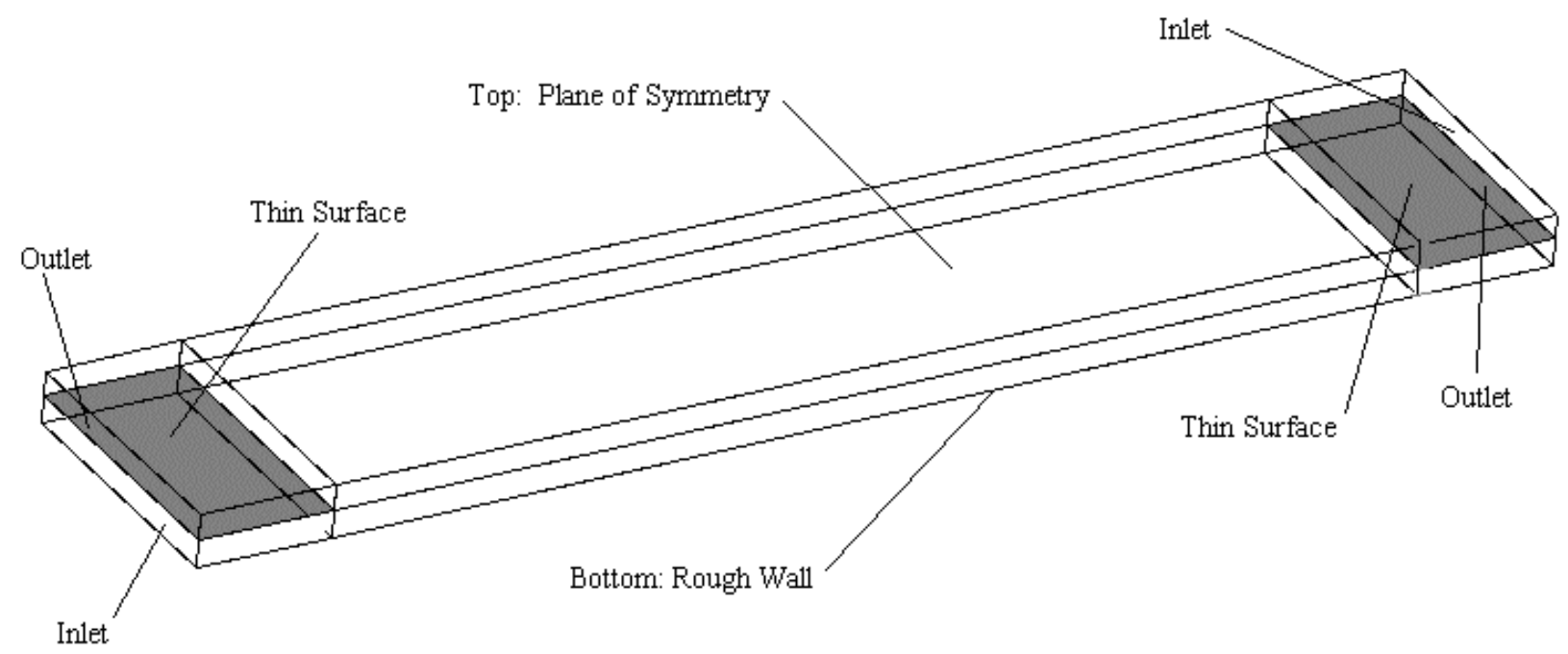

Figure 5.4 - Geometry used in CFX for simulation of Sullivan and List experiments.

A medium grid consisting of 450x50 cells and a fine grid consisting of 600x80 cells, were used for the simulations. In the main shear region, between the splitter plates, the medium grid consisted of 350 cells in the longitudinal direction by 50 cells in the vertical direction. Here the cells were concentrated towards the lower wall and vertical center of the model using a geometric progression factor of 1.05 . This gave the smallest cell at the interface dimensions of approximately $1.0 \mathrm{~cm}$ long and $2.0 \mathrm{~mm}$ high. In the main shear region the fine grid consisted of 500 cells in the longitudinal direction by 80 cells in the vertical direction, and a geometric progression factor of 1.04 was used in the vertical direction. The smallest cell size here was approximately $8.0 \mathrm{~mm}$ long by $1.0 \mathrm{~mm}$ high.

All simulations were performed as two-dimensional, turbulent flows. SFST1, as is described in Section 3.3, was used to model the mixing of the two fluids. This model was selected based on the assumption that the two fluids would be governed by essentially the 
same velocity field, since they are miscible fluids. Turbulence effects were modeled using the $k-\varepsilon$ turbulence model with the added buoyancy terms as described in Section 4.3. Boundary conditions for the inlets were modeled by setting a fixed velocity such that the inlet flow rates were the same as those in the experiments. Specified values of the volume fractions corresponding to the pure unmixed fluids were also set at the inlets. Values of $k$ and $\varepsilon$ were estimated at the inlets using

$$
k_{\text {inl }}=\mathrm{c}_{\mathrm{p} 1} \mathrm{U}_{\mathrm{inl}}^{2}
$$

and

$$
\varepsilon_{\text {inl }}=\frac{k_{\text {inl }}}{c_{\mathrm{p} 2} D_{\mathrm{h}}}
$$

where $c_{\mathrm{p} 1}$ and $\mathrm{c}_{\mathrm{p} 2}$ are empirical constants with values of 0.002 and 0.3 respectively, and $\mathrm{D}_{\mathrm{h}}$ is the hydraulic diameter. The resulting turbulence intensity in the RMS fluctuations as given by Eq. (5.2.1) is approximately $5 \%$ of the inlet velocity. These formulas were used since the actual turbulence intensity in the experiments was not available. As most of the turbulence is generated by the shear at the interface in the present flow, the inlet values of $k$ and $\varepsilon$ as given by Eq. (5.2.1) and (5.2.2) did not have a noticeable influence on the predicted parameters. Hence Dirichlet boundary conditions were specified on all quantities at the inlets except pressure, which was extrapolated from downstream..

Boundary conditions at the outlets were set as if the fluid was exiting the domain with a free surface at the top of the outlet boundary in the longitudinal direction. This boundary condition corresponds to atmospheric pressure at the top of the outlet boundary. For 
stratified flows exiting the domain in the longitudinal direction, perpendicular to the gravity vector, the discretised pressure for this condition is set by

$$
p_{j}=p_{j+1}+\int_{j}^{j+1}\left(\rho_{m}-\rho_{R e f}\right)(g) d y
$$

where the sign of the gravitational acceleration has been taken into account and acts downward and the $\mathrm{j}$ index indicates the vertical direction. Here $\rho_{\text {Ref }}$ is a reference density in CFX, which was set equal to the average of the unmixed phase densities. The boundary condition represented by Eq. (5.2.3) was expected to be accurate for the upper outlet, where the flow exits with atmospheric pressure at the top of the outlet. The lower outlet was expected to be approximately described by this condition, in light of the fact that the pressure was probably slightly different than atmospheric at the lower outlet. Boundary conditions for all other quantities at the outlets were modeled by setting a zero derivative condition. Quantities as set at the inlets for each case are listed in Table 5.2.

Table 5.2 - Inlet Flow Parameters for CFX model

Lower inlet parameters

$\begin{array}{cccccc}\text { Case } & \mathrm{U}(\mathrm{cm} / \mathrm{s}) & \mathrm{V}(\mathrm{cm} / \mathrm{s}) & \rho\left(\mathrm{kg} / \mathrm{m}^{3}\right) & k\left(\mathrm{~cm}^{2} / \mathrm{s}^{2}\right) & \varepsilon\left(\mathrm{cm}^{2} / \mathrm{s}^{3}\right) \\ 1 & 3.682 & 0.00 & 1019.00 & 0.0271 & 0.0098 \\ 10 & 3.874 & 0.00 & 1013.00 & 0.0300 & 0.0108 \\ 11 & 3.874 & 0.00 & 1008.00 & 0.0300 & 0.0108 \\ 13 & 3.874 & 0.00 & 1019.00 & 0.0300 & 0.0108\end{array}$

Upper Inlet parameters

$\begin{array}{cccccc}\text { Case } & \mathrm{U}(\mathrm{cm} / \mathrm{s}) & \mathrm{V}(\mathrm{cm} / \mathrm{s}) & \rho\left(\mathrm{kg} / \mathrm{m}^{3}\right) & k\left(\mathrm{~cm}^{2} / \mathrm{s}^{2}\right) & \varepsilon\left(\mathrm{cm}^{2} / \mathrm{s}^{3}\right) \\ 1 & 4.213 & 0.00 & 1000.00 & 0.0355 & 0.0128 \\ 10 & 4.297 & 0.00 & 1000.00 & 0.0369 & 0.0133 \\ 11 & 2.856 & 0.00 & 1000.00 & 0.0163 & 0.0059 \\ 13 & 4.297 & 0.00 & 1000.00 & 0.0369 & 0.0133\end{array}$


The free surface was modeled using a plane of symmetry. Certain aspects of the free surface were neglected with this choice of boundary condition; though they are present, their influence on the overall flow was expected to be small. The bottom wall was modeled as a fully rough wall, where the law of the wall given by

$$
\mathrm{U}^{+}=2.44 \ln \left(\frac{\mathrm{y}}{\mathrm{k}_{\mathrm{s}}}\right)+8.5
$$

was used to implement the boundary condition on the velocity and the turbulence quantities. Here $\mathrm{k}_{\mathrm{S}}$ is the average roughness height, which was set equal to $0.75 \mathrm{~cm}$ in accordance with the rocky bottom in Sullivan and List's experiments. The reason for modifying the law of the wall from its default form in CFX, was to obtain a similar velocity profile as encountered in the experiments, not necessarily to increase the free stream turbulence or turbulence that would influence the mixing at the interface.

The QUICK scheme was used to discretise the velocity components; this scheme should give at least second order accuracy on the grids in the present simulations. The volume fraction equation was discretised using first order upwinding as is recommended in the CFX Users Manual [6]. Pressure was calculated using central differencing, while the turbulence quantities were calculated using Hybrid differencing. All simulations were run using transient marching to steady state. Typically 30 outer iterations were performed over about 500 time steps of 1.0 second, which corresponded to approximately three flow-through times. At this time the outlet flow rates and vertical profiles of all the quantities remained constant with continued iteration and the solution was taken as the steady state. 


\subsection{Results and Discussion}

As previously mentioned, four cases from Sullivan and List's experiments were simulated, corresponding to their experiments numbered $1,10,11$, and 13 . In the experiments, the interfacial mixing for cases 1 and 13 was characterized as belonging to the shear driven wave breaking regime. For cases 10 and 11 in the experiments, interfacial mixing was characterized by Kelvin-Helmholtz instabilities. In the experiments the data used to calculate the desired flow parameters was taken along a vertical line at different locations in the longitudinal direction for each case. The data for case 1 was taken approximately $350.0 \mathrm{~cm}$ from the left splitter plate, while the data for cases 10 and 11 was taken approximately $50.0 \mathrm{~cm}$ from the left splitter plate. The data for case 13 was taken approximately $200.0 \mathrm{~cm}$ from the left splitter plate. In the simulations the data was taken at similar locations, corresponding to the appropriate case. Longitudinal averages of measured parameters were also taken in the simulations; these averages were taken between 50.0 and $350.0 \mathrm{~cm}$ from the left splitter plate. For each case, predicted values from the simulations and parameters that characterize the flow are given in Tables 5.3 through 5.6, along with the experimental values in the right hand column. Input parameters for densities and flow rates, and the buoyancy for each case are also given. The multiphase model and the turbulence model are listed at the top of the columns for each case, and the longitudinally averaged values are indicated by the term (LA) in the grid column. The parameters given by the medium and fine grid solutions do not vary significantly for most of the parameters, implying the solutions are close to grid independence for the present models. 
Table 5.3 - Computational results and experimental values for Case 1

Input Parameters

$$
\begin{array}{rll}
\rho_{\mathrm{O}} & =1019.00 & \mathrm{~kg} / \mathrm{m}^{3} \\
\rho_{1} & =1000.00 \mathrm{~kg} / \mathrm{m}^{3} \\
\mathrm{H}_{\mathrm{O}} & =10.00 \mathrm{~cm} \\
\mathrm{H}_{1} & =9.25 & \mathrm{~cm} \\
\mathrm{Q}_{\mathrm{O}} & =36.82 & \mathrm{~cm}^{2} / \mathrm{s} \\
\mathrm{Q}_{1} & =38.97 & \mathrm{~cm} / \mathrm{s} \\
\mathrm{U}_{\mathrm{O} \text { (inlet) }} & =3.682 & \mathrm{~cm} / \mathrm{s} \\
\mathrm{U}_{1 \text { (inlet) }} & =4.213 & \mathrm{~cm} / \mathrm{s} \\
\mathrm{b} & =18.64 & \mathrm{~cm} / \mathrm{s}^{2}
\end{array}
$$

\begin{tabular}{|c|c|c|c|c|c|c|}
\hline \multicolumn{3}{|c|}{ Computational } & \multicolumn{4}{|c|}{ Experimental } \\
\hline MP Model: & SFST1 & SFST1 & SFST1 & SFST1 & - & \\
\hline Grid: & Medium & Fine & $\operatorname{Med}(\mathrm{LA})$ & Fine $(\mathrm{LA}) *$ & - & \\
\hline Turb Model: & $\mathrm{k}-\varepsilon$ & $\mathrm{k}-\varepsilon$ & $\mathrm{k}-\varepsilon$ & $\mathrm{k}-\varepsilon$ & - & \\
\hline $\mathrm{Q}_{2}=$ & 37.539 & 37.86 & 37.54 & 37.86 & 42.29 & $\mathrm{~cm}^{2} / \mathrm{s}$ \\
\hline $\mathrm{Q}_{3}=$ & 38.254 & 37.93 & 38.25 & 37.93 & 33.50 & $\mathrm{~cm}^{2} / \mathrm{s}$ \\
\hline $\mathrm{h}_{\mathrm{O}}=$ & 2.8355 & 2.9136 & 2.4041 & 2.4374 & 4.14 & $\mathrm{~cm}$ \\
\hline $\mathrm{h}_{1}=$ & 2.4688 & 2.2446 & 3.3942 & 3.4641 & 4.66 & $\mathrm{~cm}$ \\
\hline $\mathrm{U}_{\mathrm{O}}=$ & 4.5036 & 4.7145 & 4.3640 & 4.3942 & 6.89 & $\mathrm{~cm} / \mathrm{s}$ \\
\hline $\mathrm{U}_{1}=$ & -3.8338 & -3.7276 & -3.9432 & -3.9616 & -2.97 & $\mathrm{~cm} / \mathrm{s}$ \\
\hline$\eta=$ & 9.0850 & 8.7955 & 9.3600 & 9.3980 & 6.17 & $\mathrm{~cm}$ \\
\hline $\mathrm{u}_{\mathrm{i}}=$ & 1.4574 & 1.3972 & 1.1646 & 1.0457 & 3.34 & $\mathrm{~cm} / \mathrm{s}$ \\
\hline$\delta=$ & 1.7160 & 1.6021 & 1.0817 & 1.0033 & 0.234 & $\mathrm{~cm}$ \\
\hline $\mathrm{du} / \mathrm{dy}_{\operatorname{Max}}=$ & 4.2453 & 5.0292 & 4.6724 & 5.3087 & 3.37 & $\mathrm{~s}^{-1}$ \\
\hline $\mathrm{h}_{\mathrm{S}}=$ & 1.9639 & 1.6786 & 1.8102 & 1.6137 & 2.93 & $\mathrm{~cm}$ \\
\hline $\mathrm{Ri}_{\mathrm{O}}=$ & 5.6953 & 4.9351 & 4.4927 & 4.1880 & 6.179 & - \\
\hline $\mathrm{Ri}_{1}=$ & 1.6436 & 1.5930 & 2.4771 & 2.6464 & 2.202 & - \\
\hline $\mathrm{Ri}_{\mathrm{S}}=$ & 0.5266 & 0.4390 & 0.4889 & 0.4310 & 0.570 & - \\
\hline $\mathrm{Ri}_{\mathrm{L}}=$ & 1.5862 & 1.5425 & 1.7339 & 1.7648 & 2.6315 & - \\
\hline $\mathrm{Ri}^{*}=$ & 5.5321 & 5.5321 & 5.5321 & 5.5321 & 5.5321 & - \\
\hline
\end{tabular}

Computational

*LA = longitudinal average

Table 5.4 - Computational results and experimental values for Case 10

Input Parameters

$$
\begin{array}{rll}
\rho_{\mathrm{O}} & =1013.00 & \mathrm{~kg} / \mathrm{m}^{3} \\
\rho_{1} & =1000.00 \mathrm{~kg} / \mathrm{m}^{3} \\
\mathrm{H}_{\mathrm{O}} & =10.00 & \mathrm{~cm} \\
\mathrm{H}_{1} & =9.25 & \mathrm{~cm} \\
\mathrm{Q}_{\mathrm{O}} & =38.74 & \mathrm{~cm}^{2} / \mathrm{s} \\
\mathrm{Q}_{1} & =39.75 & \mathrm{~cm}^{2} / \mathrm{s} \\
\mathrm{U}_{\mathrm{O} \text { (inlet) }} & =3.874 & \mathrm{~cm} / \mathrm{s} \\
\mathrm{U}_{1 \text { (inlet) }} & =4.297 & \mathrm{~cm} / \mathrm{s} \\
\mathrm{b} & =12.75 & \mathrm{~cm} / \mathrm{s}^{2}
\end{array}
$$

\section{Computational}

$\begin{array}{rcccccl}\text { MP Model: } & \text { SFST1 } & \text { SFST1 } & \text { SFST1 } & \text { SFST1 } & - & \\ \text { Grid: } & \text { Medium } & \text { Fine } & \text { Med }(\mathrm{LA}) & \text { Fine }(\mathrm{LA}) & - & \\ \text { Turb Model: } & \mathrm{k}-\varepsilon & \mathrm{k}-\varepsilon & \mathrm{k}-\varepsilon & \mathrm{k}-\varepsilon & - & \\ \mathrm{Q}_{2}= & 39.69 & 40.15 & 39.69 & 40.15 & 41.07 & \mathrm{~cm}^{2} / \mathrm{s} \\ \mathrm{Q}_{3}= & 38.81 & 38.35 & 38.81 & 38.35 & 37.42 & \mathrm{~cm}^{2} / \mathrm{s} \\ \mathrm{h}_{\mathrm{O}}= & 2.8169 & 2.7653 & 2.8931 & 2.9083 & 6.12 & \mathrm{~cm} \\ \mathrm{~h}_{1}= & 3.5430 & 3.8190 & 3.3058 & 3.3548 & 4.45 & \mathrm{~cm} \\ \mathrm{U}_{\mathrm{O}}= & 4.1109 & 4.1280 & 4.4303 & 4.5142 & 3.89 & \mathrm{~cm} / \mathrm{s} \\ \mathrm{U}_{1}= & -4.4939 & -4.5305 & -4.1853 & -4.1629 & -4.59 & \mathrm{~cm} / \mathrm{s} \\ \eta= & 10.4040 & 10.4761 & 9.7310 & 9.6740 & 10.59 & \mathrm{~cm} \\ \mathrm{u}_{\mathrm{i}}= & -0.8714 & -0.8142 & 0.1469 & 0.1658 & -0.04 & \mathrm{~cm} / \mathrm{s} \\ \delta= & 2.7895 & 2.9196 & 2.9196 & 2.8884 & 0.667 & \mathrm{~cm} \\ \mathrm{du} / \mathrm{dy}_{\mathrm{Max}}= & 4.0909 & 4.5341 & 3.4008 & 3.9470 & 3.820 & \mathrm{~s} \\ \mathrm{~h}_{\mathrm{S}}= & 2.1034 & 1.9097 & 2.5726 & 2.2449 & 2.22 & \mathrm{~cm} \\ \mathrm{Ri}_{\mathrm{O}}= & 1.4471 & 1.4438 & 2.1171 & 2.0544 & 5.060 & - \\ \mathrm{Ri}_{1}= & 3.4433 & 3.5266 & 2.4115 & 2.4427 & 2.744 & - \\ \mathrm{Ri}_{\mathrm{S}}= & 0.3623 & 0.3249 & 0.4418 & 0.3798 & 0.390 & - \\ \mathrm{Ri}_{\mathrm{L}}= & 1.2147 & 1.2576 & 1.1840 & 1.1962 & 2.0189 & - \\ \mathrm{Ri}^{*}= & 3.5335 & 3.5335 & 3.5335 & 3.5335 & 3.5335 & -\end{array}$


Table 5.5 - Computational results and experimental values for Case 11

\section{Input Parameters}

$$
\begin{array}{rll}
\rho_{\mathrm{O}} & =1008.00 & \mathrm{~kg} / \mathrm{m}^{3} \\
\rho_{1} & =1000.00 & \mathrm{~kg} / \mathrm{m}^{3} \\
\mathrm{H}_{\mathrm{O}} & =10.00 & \mathrm{~cm} \\
\mathrm{H}_{1} & =9.25 & \mathrm{~cm} \\
\mathrm{Q}_{\mathrm{O}} & =38.74 & \mathrm{~cm}^{2} / \mathrm{s} \\
\mathrm{Q}_{1} & =26.42 & \mathrm{~cm} / \mathrm{s} \\
\mathrm{U}_{\mathrm{O} \text { (inlet) }} & =3.874 & \mathrm{~cm} / \mathrm{s} \\
\mathrm{U}_{1 \text { (inlet) }} & =2.856 & \mathrm{~cm} / \mathrm{s} \\
\mathrm{b} & =7.85 & \mathrm{~cm} / \mathrm{s}^{2}
\end{array}
$$

\section{Computational}

$\begin{array}{rcccccl}\text { MP Model: } & \text { SFST1 } & \text { SFST1 } & \text { SFST1 } & \text { SFST1 } & - & \\ \text { Grid: } & \text { Medium } & \text { Fine } & \text { Med (LA) } & \text { Fine }(L A) & - & \\ \text { Turb Model: } & \mathrm{k}-\varepsilon & \mathrm{k}-\varepsilon & \mathrm{k}-\varepsilon & \mathrm{k}-\varepsilon & - & \\ \mathrm{Q}_{2}= & 40.61 & 40.48 & 40.61 & 40.48 & 41.07 & \mathrm{~cm}^{2} / \mathrm{s} \\ \mathrm{Q}_{3}= & 24.56 & 24.69 & 24.56 & 24.69 & 24.08 & \mathrm{~cm}^{2} / \mathrm{s} \\ \mathrm{h}_{\mathrm{O}}= & 2.7084 & 2.7925 & 2.9811 & 3.0732 & 4.78 & \mathrm{~cm} \\ \mathrm{~h}_{1}= & 3.7885 & 3.7632 & 3.7536 & 3.7340 & 3.2 & \mathrm{~cm} \\ \mathrm{U}_{\mathrm{O}}= & 4.0680 & 4.0408 & 4.3374 & 4.2845 & 3.47 & \mathrm{~cm} / \mathrm{s} \\ \mathrm{U}_{1}= & -3.1015 & -3.1145 & -2.9059 & -2.9304 & -3.56 & \mathrm{~cm} / \mathrm{s} \\ \eta= & 10.7315 & 10.7672 & 10.1340 & 10.2150 & 11.83 & \mathrm{~cm} \\ \mathrm{u}_{\mathrm{i}}= & -0.0694 & -0.1209 & 0.6759 & 0.5496 & 0.78 & \mathrm{~cm} / \mathrm{s} \\ \delta= & 2.8182 & 2.8449 & 3.2762 & 3.2865 & 0.819 & \mathrm{~cm} \\ \mathrm{du} / \mathrm{dy}_{\mathrm{Max}=} & 2.6204 & 2.8389 & 2.2747 & 2.5117 & 6.01 & \mathrm{~s}-1 \\ \mathrm{~h}_{\mathrm{S}}= & 2.7360 & 2.5204 & 3.2122 & 2.8953 & 1.17 & \mathrm{~cm} \\ \mathrm{Ri}_{\mathrm{O}}= & 1.2417 & 1.2654 & 1.8005 & 1.7620 & 4.941 & - \\ \mathrm{Ri}_{1}= & 3.2339 & 3.2954 & 2.4012 & 2.4965 & 1.270 & - \\ \mathrm{Ri}_{\mathrm{S}}= & 0.4177 & 0.3863 & 0.4798 & 0.4360 & 0.180 & - \\ \mathrm{Ri}_{\mathrm{L}}= & 1.1257 & 1.1358 & 1.1669 & 1.1794 & 1.3826 & - \\ \mathrm{Ri}^{*}= & 3.2053 & 3.2053 & 3.2053 & 3.2053 & 3.2053 & -\end{array}$

\section{Experimental}

Table 5.6 - Computational results and experimental values for Case 13

\section{Input Parameters}

$$
\begin{array}{rll}
\rho_{\mathrm{O}} & =1019.00 & \mathrm{~kg} / \mathrm{m}^{3} \\
\rho_{1} & =1000.00 & \mathrm{~kg} / \mathrm{m}^{3} \\
\mathrm{H}_{\mathrm{O}} & =10.00 & \mathrm{~cm} \\
\mathrm{H}_{1} & =9.25 & \mathrm{~cm} \\
\mathrm{Q}_{\mathrm{O}} & =38.74 & \mathrm{~cm}^{2} / \mathrm{s} \\
\mathrm{Q}_{1} & =39.75 & \mathrm{~cm}^{2} / \mathrm{s} \\
\mathrm{U}_{\mathrm{O} \text { (inlet) }} & =3.874 & \mathrm{~cm} / \mathrm{s} \\
\mathrm{U}_{1 \text { (inlet) }} & =4.297 & \mathrm{~cm} / \mathrm{s} \\
\mathrm{b} & =18.64 & \mathrm{~cm} / \mathrm{s}^{2}
\end{array}
$$

\section{Computational}

$\begin{array}{rcccccl}\text { MP Model: } & \text { SFST1 } & \text { SFST1 } & \text { SFST1 } & \text { SFST1 } & - & \\ \text { Grid: } & \text { Medium } & \text { Fine } & \text { Med (LA) } & \text { Fine }(L A) & - & \\ \text { Turb Model: } & \mathrm{k}-\varepsilon & \mathrm{k}-\varepsilon & \mathrm{k}-\varepsilon & \mathrm{k}-\varepsilon & - & \\ \mathrm{Q}_{2}= & 39.78 & 38.45 & 39.78 & 38.45 & 40.67 & \mathrm{~cm}^{2} / \mathrm{s} \\ \mathrm{Q}_{3}= & 38.71 & 40.05 & 38.71 & 40.05 & 37.82 & \mathrm{~cm}^{2} / \mathrm{s} \\ \mathrm{h}_{\mathrm{O}}= & 2.3185 & 2.3745 & 2.3602 & 2.3046 & 4.84 & \mathrm{~cm} \\ \mathrm{~h}_{1}= & 3.6526 & 3.5882 & 3.3736 & 3.6435 & 4.31 & \mathrm{~cm} \\ \mathrm{U}_{\mathrm{O}}= & 4.6495 & 4.7463 & 4.5597 & 4.6690 & 4.99 & \mathrm{~cm} / \mathrm{s} \\ \mathrm{U}_{1}= & -3.9973 & -3.95026 & -4.0833 & -4.0186 & -3.58 & \mathrm{~cm} / \mathrm{s} \\ \eta= & 9.3059 & 9.1874 & 9.4950 & 9.3500 & 8.13 & \mathrm{~cm} \\ \mathrm{u}_{\mathrm{i}}= & 1.5288 & 1.47059 & 1.1634 & 1.3447 & 1.98 & \mathrm{~cm} / \mathrm{s} \\ \delta= & 1.1729 & 1.0732 & 1.1431 & 1.2144 & 0.180 & \mathrm{~cm} \\ \mathrm{du} / \mathrm{dy}_{\mathrm{Max}}= & 4.4264 & 5.4075 & 4.7966 & 5.4066 & 4.76 & \mathrm{~s} \\ \mathrm{~h}_{\mathrm{S}}= & 1.9535 & 1.6082 & 1.8358 & 1.6367 & 1.80 & \mathrm{~cm} \\ \mathrm{Ri}_{\mathrm{O}}= & 4.4374 & 4.1246 & 4.0451 & 4.0199 & 10.130 & - \\ \mathrm{Ri}_{1}= & 2.2294 & 2.2760 & 2.4212 & 2.4418 & 2.640 & - \\ \mathrm{Ri}_{\mathrm{S}}= & 0.4870 & 0.3963 & 0.4584 & 0.4044 & 0.460 & - \\ \mathrm{Ri}_{\mathrm{L}}= & 1.6668 & 1.6645 & 1.6006 & 1.6604 & 2.5542 & - \\ \mathrm{Ri}^{*}= & 5.1643 & 5.1643 & 5.1643 & 5.1643 & 5.1643 & -\end{array}$


While the data that is presented in Tables 5.4 through 5.7 shows that the model predicts most of the quantities as reported by Sullivan and List reasonably accurately, each of the predicted quantities will be discussed in light of the experimental values. Reasons for any discrepancy in the predicted and experimental values will be investigated in light of any uncertainty in the boundary conditions, numerics, computational models, and experimental conditions. As will be considered, the concentration boundary layers and layer Richardson numbers were possibly influenced by some of the boundary conditions, which were estimated in the simulations. Also, interfacial parameters such as the interfacial thickness, $\delta$, and the maximum velocity gradient thickness, $\mathrm{h}_{\mathrm{S}}$, were highly influenced by the buoyancy terms in the form of the $k-\varepsilon$ model that was used. Hence, the $k-\varepsilon$ model is discussed in light of the turbulence kinetic energy budget at the mixed interface, specifically with regard to the models performance at a turbulent/non-turbulent interface. Overall, reasonably good trends (not necessarily magnitudes) will be shown for $\delta, \delta_{\mathrm{M}}, \mathrm{Ri}_{\mathrm{L}}$, and $\mathrm{E}$ as estimated from the longitudinally averaged values.

Considering the concentration boundary layers, $\mathrm{h}_{\mathrm{O}}$ and $\mathrm{h}_{1}$, one possible reason that these parameters were predicted lower in magnitude than the experimental values is in how $\mathrm{h}_{0}$ and $\mathrm{h}_{1}$ were defined. The definition of the concentration boundary layers was based on the predicted time averaged volume fraction of the lighter phase, $\mathrm{r}_{\alpha}$, being either 0.99 or 0.01. To enlarge the magnitude it may have been sufficient to define $h_{O}$ and $h_{1}$ in some other manner; however, the values as given by $0.99 r_{\alpha}$ and $0.01 r_{\alpha}$ were taken as reasonable predictions, given Sullivan and List's definition. 
Considering a more important trend, in the simulations the relative magnitudes of $h_{0}$ an $h_{1}$ were opposite to those found in the experiments for all four cases. In investigating why this is so, the concentration boundary layers will be discussed in light of the velocity boundary layers that comprise the shear layer. The upper velocity boundary layer begins forming at the upper, right inlet and the lower velocity boundary layer begins forming at the lower, left inlet. Since both the upper and lower layers have approximately the same inlet Reynolds number for all of the cases, the velocity boundary layers at the interface tend to grow with the same magnitude in the longitudinal direction. In light of this, the values for $h_{O}$ and $h_{1}$ that are given by the simulations tend to support what would be expected if $h_{O}$ and $h_{1}$ behaved in a similar manner as the velocity boundary layers. For instance in case 1 , where the data was taken near the right inlet, $h_{1}$ was greater than $h_{O}$ in the experiments, where it would seem that $h_{1}$ should be less than $h_{O}$ as predicted in the simulations. Near the right inlet where the data was taken, the upper shear layer has only been developing for $50.0 \mathrm{~cm}$ and the lower shear layer has been developing for $350.0 \mathrm{~cm}$. For cases 10 and 11 , where the data was taken near the left inlet, $h_{1}$ was greater than $h_{0}$ in the simulations, and the opposite was observed in the experiments.

These discrepancies can be explained in part by considering that in the experiments the interfacial offset was larger than was predicted in the simulations. The interfacial offset is defined as the distance between the concentration interface, $\eta$, and the velocity interface, the vertical location where the velocity is zero in the shear layer. This interfacial offset causes the concentration boundary layers to attain different magnitudes than would be expected by assuming that the relative magnitudes of the concentration 
boundary layers are similar to the velocity boundary layers. Also, the velocity interface and concentration interface occurred at slightly higher vertical locations in the experiments, than in the simulations. As can be inferred from Sullivan and List's data for $\eta$ and $\mathrm{u}_{\mathrm{i}}$, in the experiments the interfacial offset tended to increase towards the right outlet. Here $\eta$ was lower than the velocity interface near the right outlet, and slightly higher than the velocity interface near the left outlet. As is indicated from the predicted values, the simulations give a similar trend for the interfacial offset; however, the predicted offset is not large enough to reverse the trend in $h_{O}$ and $h_{1}$ as seems to happen in the experiments. Figure 5.5 shows the predicted variation of the concentration interface and of the velocity interface in the longitudinal direction for case 11.

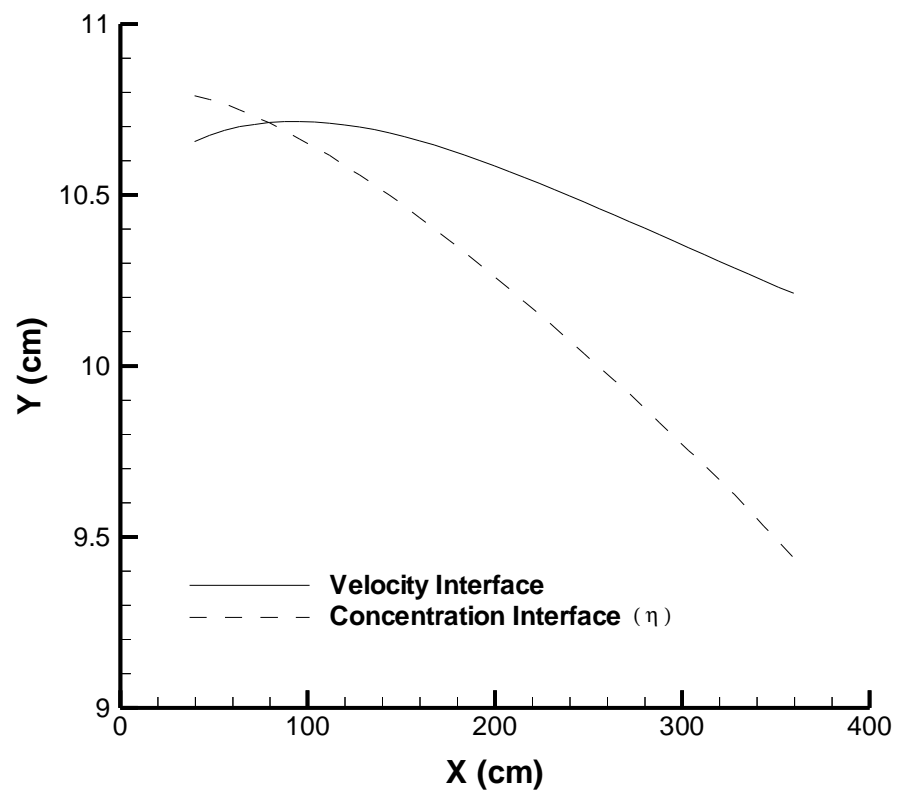

Figure 5.5 - Position of velocity and concentration interface for case 11. 
For cases 10 and 11, where the data was taken close to the left inlet, the velocity interface situates itself at a higher vertical location in the experiments than in the simulations. In the experiments, the velocity interface rises as much as $2.0 \mathrm{~cm}$ above the left splitter plate and essentially acts as a moving boundary for the lower velocity boundary layer. Since the ability of the velocity interface to move effectively widens the lower part of the shear layer, it may have contributed to the lower concentration boundary layer being higher in the experiments than in the simulations. Also, near the left inlet it is possible that the amount of free stream turbulence entering the domain was actually higher than as estimated by Eq. (5.2.1). In the experiments this inlet turbulence may have persisted for some time downstream and $50.0 \mathrm{~cm}$ from the left splitter plate may have influenced $\mathrm{h}_{0}$, making it larger than as predicted by the local mean shear for cases 10 and 11. At this position the upper layer was most probably free of history effects.

The discrepancies in the flow field that influenced the concentration boundary layers may have been a result of estimating some of the unknown boundary conditions. In the simulations, the inlet velocity profiles were uniform which is not realistic. The inlet turbulence intensity and the dynamic pressures at the outlets were also unknown. Hence the turbulence intensities were estimated and the dynamic pressures at the outlets were set equal to zero for both the upper and lower outlets. The actual relative difference in pressures at these outlets would probably have determined the overall flow field more accurately and hence the location of the velocity interfaces more accurately. 
Even with the discrepancy in the concentration boundary layers, the predictions for the trends for the interface velocity, $\mathrm{u}_{\mathrm{i}}$, and for the concentration interface, $\eta$, are correct, though their magnitudes do not exactly agree with the experiments. Hence, because of the discrepancy in the concentration boundary layers and $\mathrm{u}_{\mathrm{i}}$, the layer Richardson numbers as predicted are somewhat difficult to compare with the experiments. The values or the layer Richardson numbers for the upper layer appear to be somewhat reasonable, when compared with the experimental values; however, the values for the lower layer Richardson numbers are different by as much as $75 \%$. In light of these uncertainties, the layer Richardson number is judged to not necessarily be the best parameter to use for evaluating the model.

Since the magnitude of the concentration boundary layer thickness is reasonably predicted, the mixed fluid thickness as is given by Eq. (5.1.9) can be used to measure the capability of the model to predict the overall mixing in the turbulent layer. Since the mixed layer Richardson number, which is given by $\mathrm{Ri}_{\mathrm{L}}$ in Eq. (5.1.13), does involve $\delta_{\mathrm{M}}$, it is taken as an appropriate parameter to measure the relevant scales of buoyancy to turbulence in the layer. To show the degree to which $\mathrm{Ri}_{\mathrm{L}}$ varied for each case, it is the plotted versus the overall Richardson number, Ri* in Figure 5.6. Ri* as was defined by Eq. (5.1.12) is the same for the simulations and the experiments by its definition. Figure 5.6 shows the ability of the model to predict the degree to which $\delta_{\mathrm{M}}$ will scale with the turbulence in the layer, for a given set of inlet flow parameters and an overall buoyancy given by $b$. 


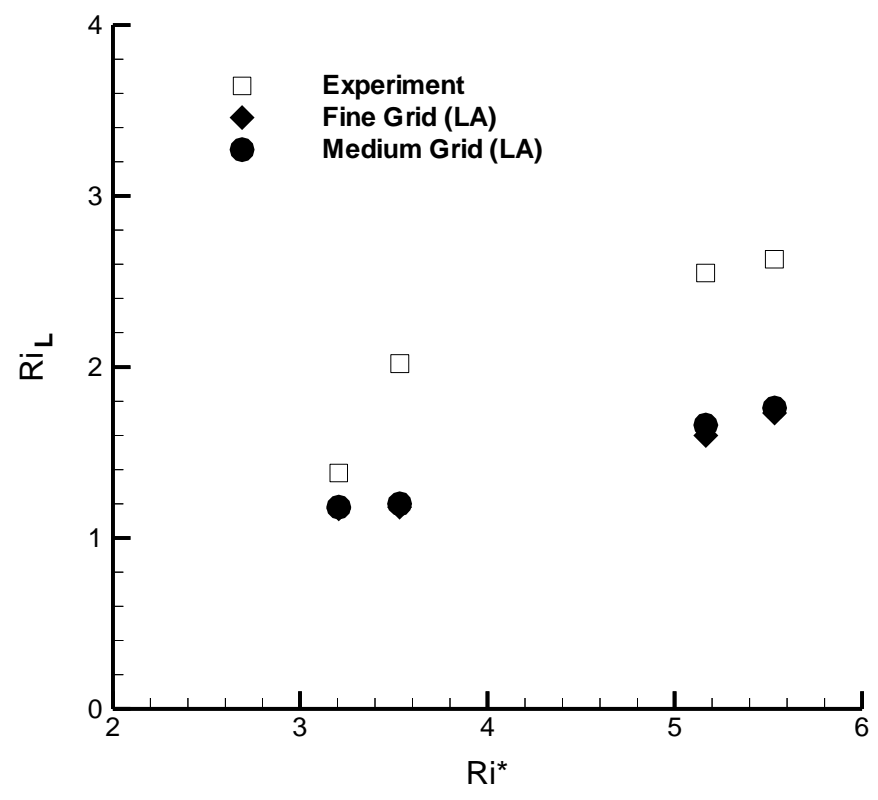

Figure 5.6 - Variation of mixed layer Richardson number with Ri*.

Though not directly given in the experimental data, another parameter related to the overall mixing in the two layers is the entrainment velocity, E, which was discussed in Section 2.1. For the simulations $\mathrm{E}$ was defined by

$$
\mathrm{E}=\frac{\mathrm{u}_{\mathrm{e}}}{\Delta \mathrm{U}}
$$

where $\Delta \mathrm{U}$ is the difference of the inlet velocities. The entrainment velocity, $\mathrm{u}_{\mathrm{e}}$, was calculated using the average of the amount of lower layer fluid flowing out of the domain above $\eta$ per unit time and the amount of upper layer fluid flowing out of the domain below $\eta$ per unit time, divided by the area over which entrainment occurred. The resulting values of E versus Ri* for each case are plotted in Figure 5.7. 


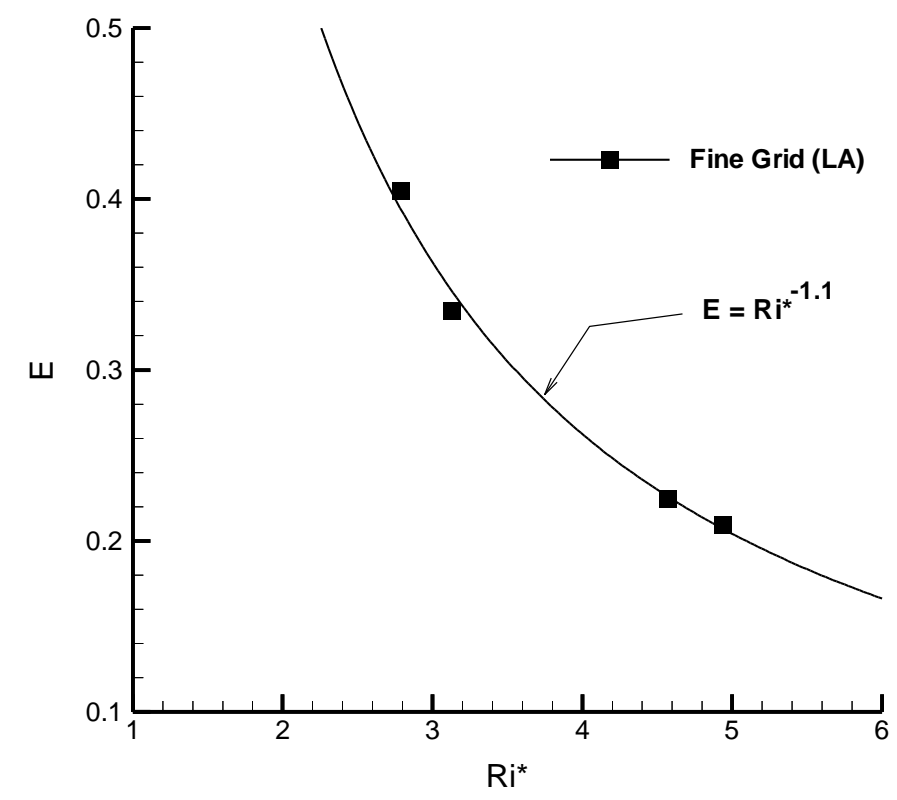

Figure 5.7 - Variation of entrainment velocity with $\mathrm{Ri}^{*}$.

Though the trend for E is not compared with any experimental data from the Sullivan and List experiments, a power law fit through the four data points does give an exponent within the experimentally reported range. The curve in Figure 5.7 follows E $\sim \mathrm{Ri}^{*}{ }^{-1.1}$. Typical experimental values for the exponent range from -0.5 to -2.0 depending on the experiment and the definition of the Richardson number [10].

Volume fraction contours and velocity vectors for each of the four cases are given in Figures 5.8 through 5.11. These figures were all taken at the center line of the simulated model and give some idea of how the increasing buoyancy tends to sharpen the interface and how the mixing occurs in the layers. 


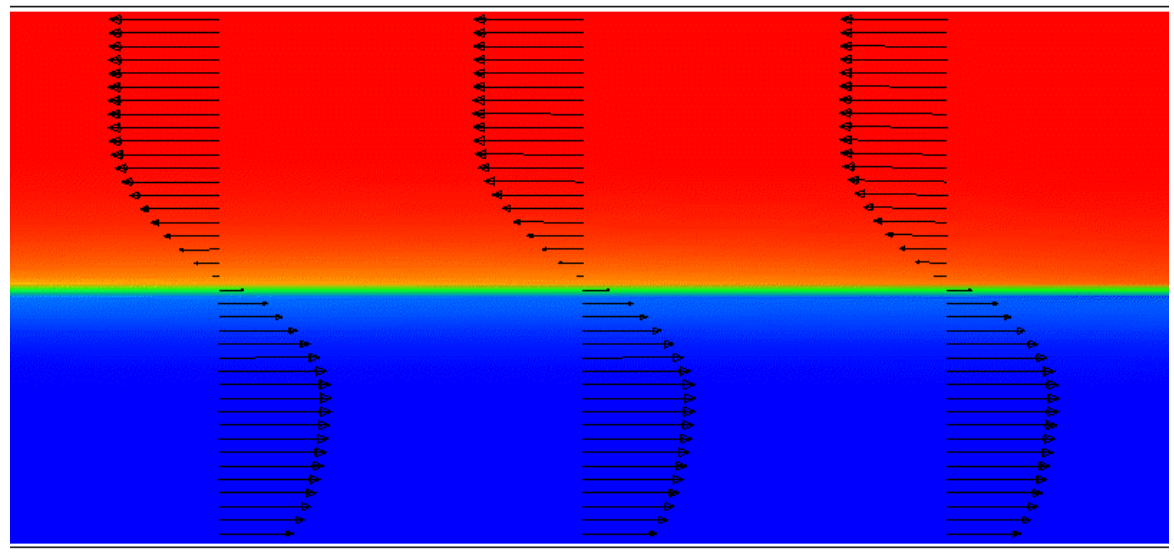

Case 1

Volume Fraction

0.00

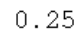

0.50

0.75

1.00

CFX

Figure 5.8 - Contours of volume fraction and velocity vectors for case 1.

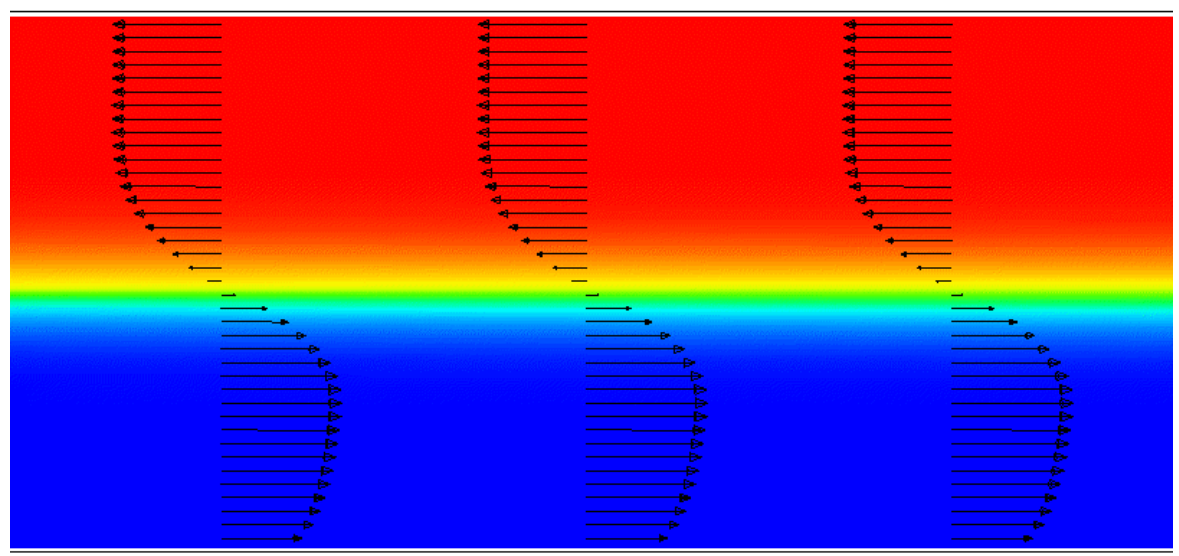

Case 10

Volume Fraction

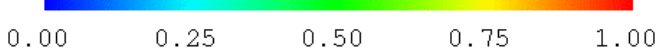

CFX

Figure 5.9 - Contours of volume fraction and velocity vectors for case 10. 


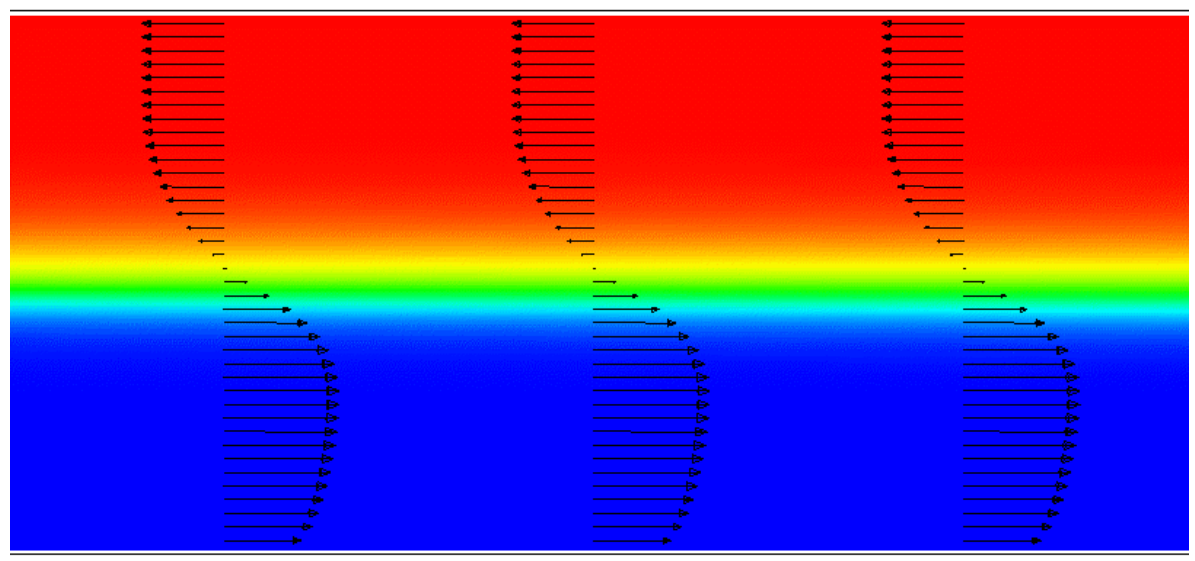

Case 11

Volume Fraction

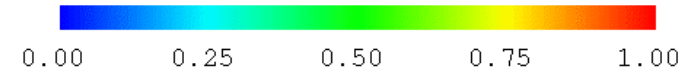

CFX

Figure 5.10 - Contours of volume fraction and velocity vectors for case 11.

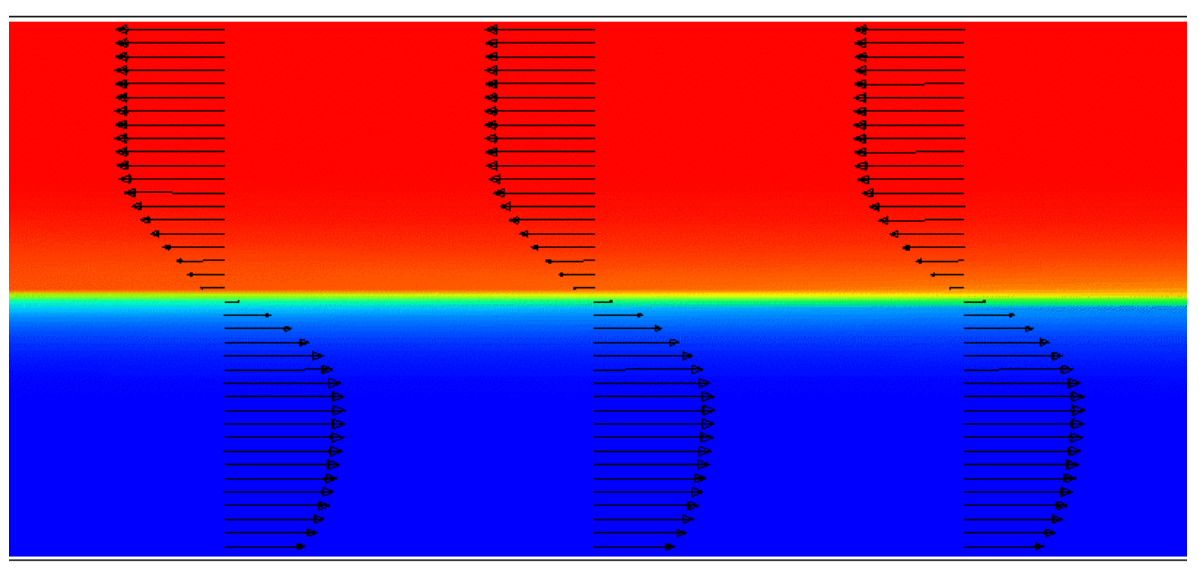

Case 13

Volume Fraction

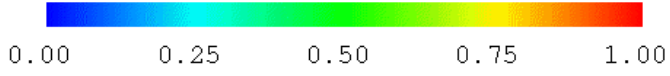

CFX

Figure 5.11 - Contours of volume fraction and velocity vectors for case 13. 
Having investigated several parameters related to the overall mixed layer and flow field, the current discussion is focused on the model's predictions regarding the behavior of the flow at the interface. Specifically, the parameters $\delta, \mathrm{h}_{\mathrm{S}}$, and $\mathrm{Ri}_{\mathrm{S}}$ and the behavior of the turbulence quantities are of interest in the interfacial region. Since these parameters are highly inter-related, the discussion will begin by considering the vertical profiles of the velocity and volume fraction at the interface. These profiles will be discussed in light of the turbulent kinetic energy budget at the interface, and the ability of the present form of the $k$ - $\varepsilon$ model to represent the relevant physics at the interface will be investigated. Finally, several trends involving $\delta, \mathrm{h}_{\mathrm{S}}$, and $\mathrm{Ri}_{\mathrm{S}}$ as predicted by the model will be compared with experimental data. Profiles for the velocity and volume fraction are given in Figures 5.12 through 5.15 for each case.

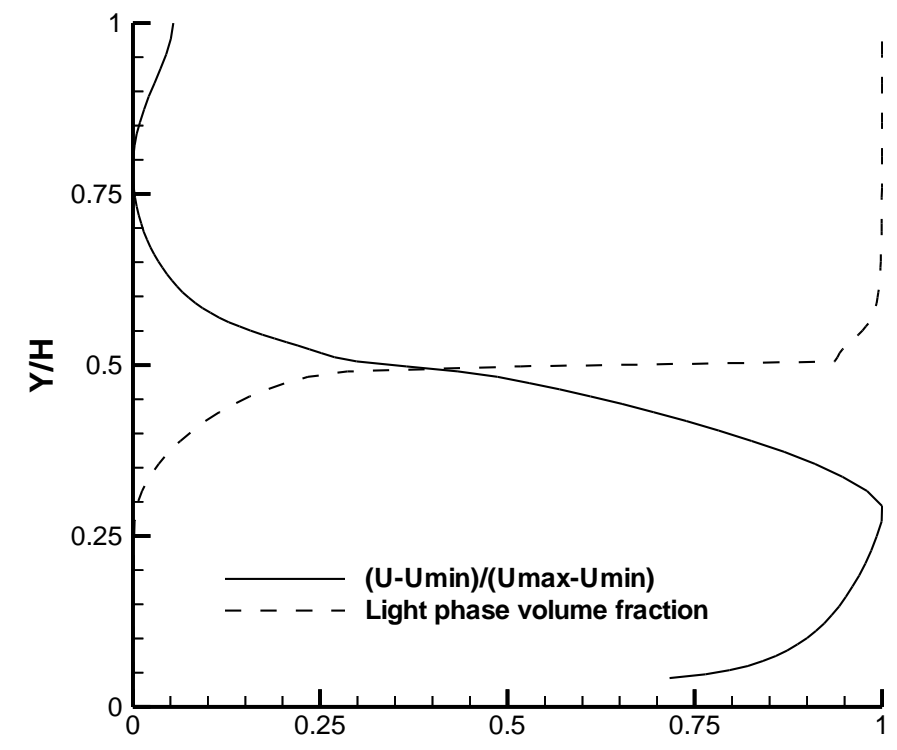

Figure 5.12 - Velocity profile and volume fraction profile for case 1. 


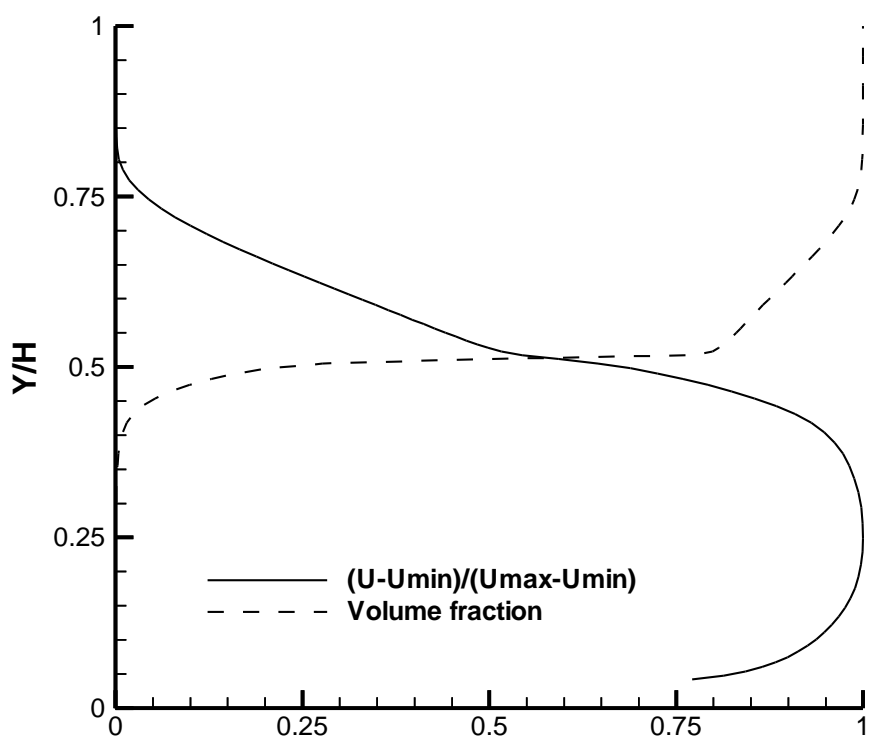

Figure 5.13 - Velocity profile and volume fraction profile for case 10.

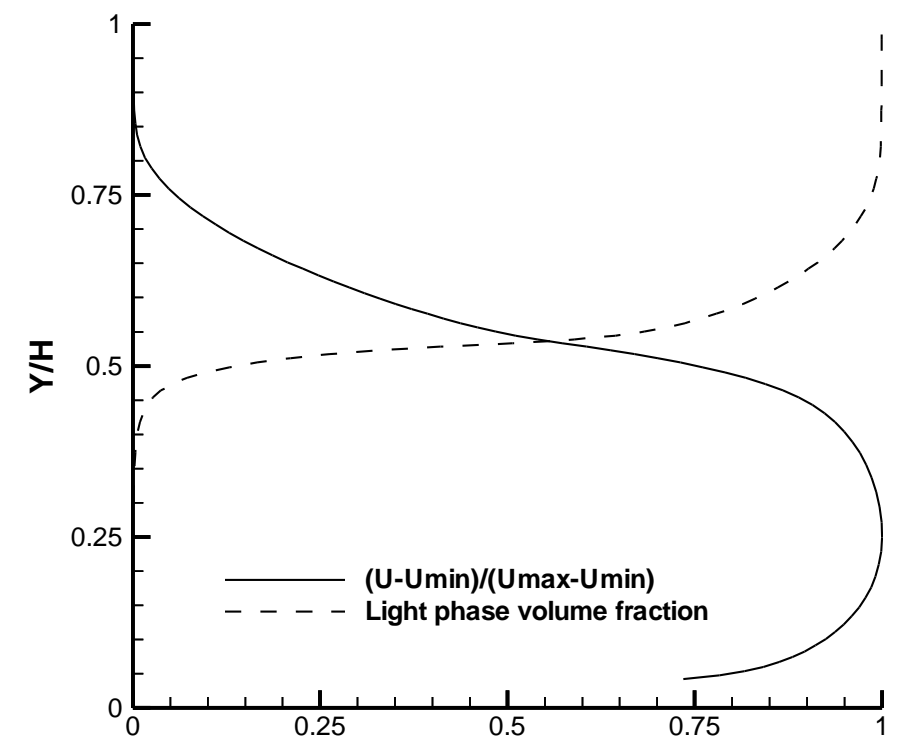

Figure 5.14 - Velocity profile and volume fraction profile for case 11. 


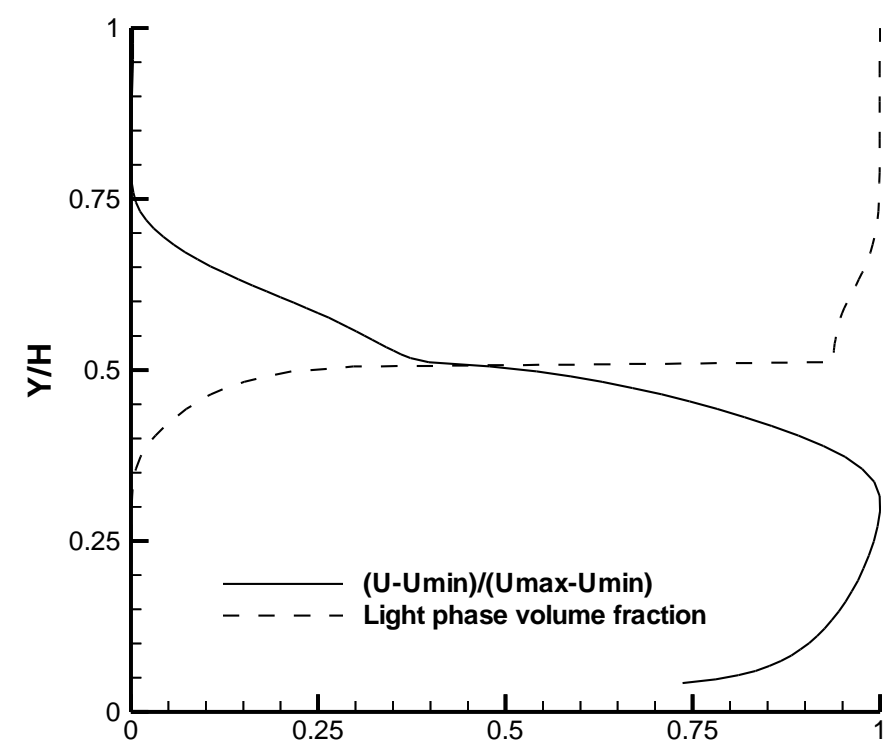

Figure 5.15 - Velocity profile and volume fraction profile for case 13.

As is evident in Figures 5.12, 5.13, and 5.15, for the cases involving higher amounts of stratification the area surrounding the interface displays a noticeable kink in the velocity and the volume fraction. The explanation for this behavior can be found by considering the form of the $k-\varepsilon$ turbulence model that was used for the simulations. For a homogeneous shear layer at steady state, the governing equation for the turbulent kinetic energy is given by

$$
-\overline{u^{\prime} v^{\prime}} \frac{\partial u}{\partial y}=+\varepsilon+\frac{g \overline{v^{\prime} \rho^{\prime}}}{\rho}
$$

where the small transport terms have been neglected. In Eq (5.3.2) the term on the left hand side is the production of $k$ by the Reynolds stress working against the mean velocity gradient. The terms on the left hand side represent the dissipation of $k$ by viscous dissipation and the dissipation of $k$ by increasing the potential energy of the flow because 
of buoyancy influences [33]. It is possible for production to balance both the viscous and buoyant dissipation locally for certain cases involving low stratification or high shear; however, with increasing buoyancy the buoyant term will suppress all turbulence at the interface [31]. Hence, except for the weakest stratified flows, the interface does tend to become laminar approaching the mixed interface.

The form of the $k-\varepsilon$ model used for the simulations is formulated based on assumptions that are typically valid for high Reynolds number turbulence. This is one of the main reasons that the standard $k-\varepsilon$ model is typically used with wall functions, since the model, without some low Reynolds number modifications, is not able to accurately resolve the features of turbulence approaching a wall or a laminar viscous layer. With the additional buoyancy source terms, the model does account for the buoyant dissipation of turbulent kinetic energy at the interface; however, the essential features of the turbulence are not accounted for. In the present form of the model, the governing turbulent kinetic energy equation at the interface is represented by

$$
\mu_{\mathrm{T}}\left(\frac{\partial \mathrm{u}}{\partial \mathrm{y}}\right)^{2}=+\varepsilon+|\mathrm{g}| \frac{\mu_{\mathrm{T}}}{\rho^{2} \sigma_{\rho}} \frac{\partial \rho}{\partial y}
$$

i.e. the production balances viscous and buoyancy dissipation. The resulting turbulent kinetic energy budget as calculated for case 13 is shown in Figure 5.16. 


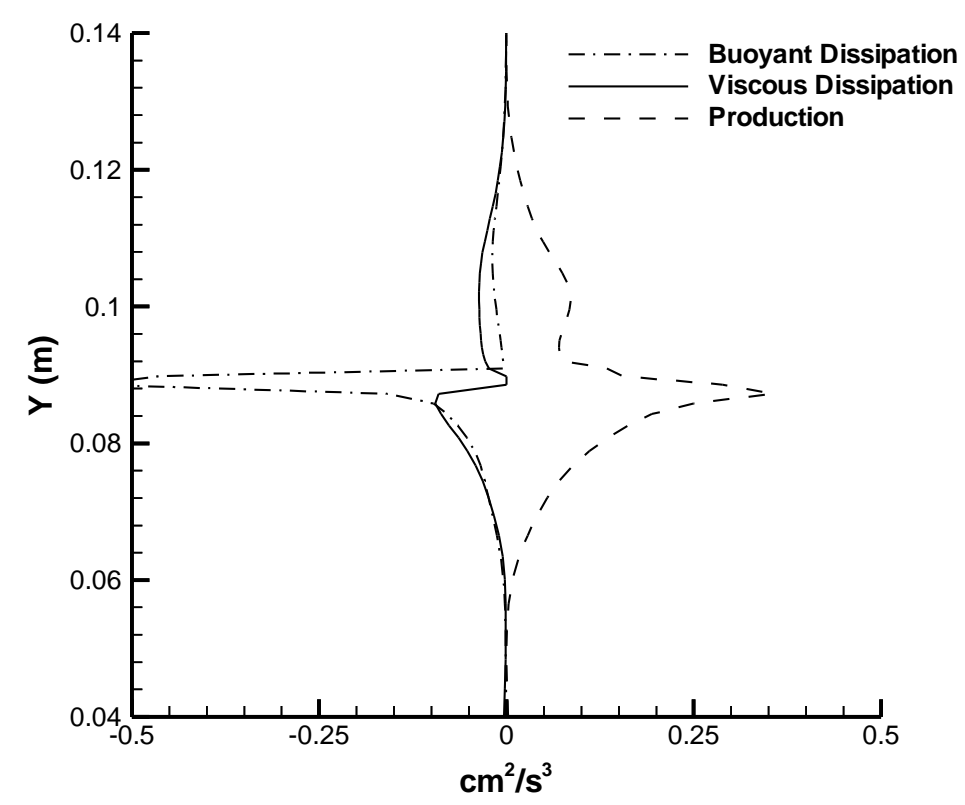

Figure 5.16 - Turbulent kinetic energy budget for case 13.

For the majority of the shear layer, production does approximately balance dissipation by viscosity and buoyancy; however, at the interface the buoyant dissipation becomes so strong that it overwhelms the production, and the viscous dissipation goes to zero. This sudden change in the buoyant dissipation and zero production at the interface give a sharp change in the interfacial parameters such as $k$. The turbulent kinetic energy profile for case 13 is given in Figure 5.17 and shows that $k$ suddenly drops to zero due to buoyant dissipation. The sink as predicted by the buoyancy term actually should tend to cause $k$ to become negative in light of the values given in Figure 5.16; however, the code essentially stops such an occurrence and sets $k$ to zero in these cases. It should also be noted that the slight rise at the bottom of the plot in Figure 5.17 is due to the diffusion from the turbulence generated at the rough wall. 


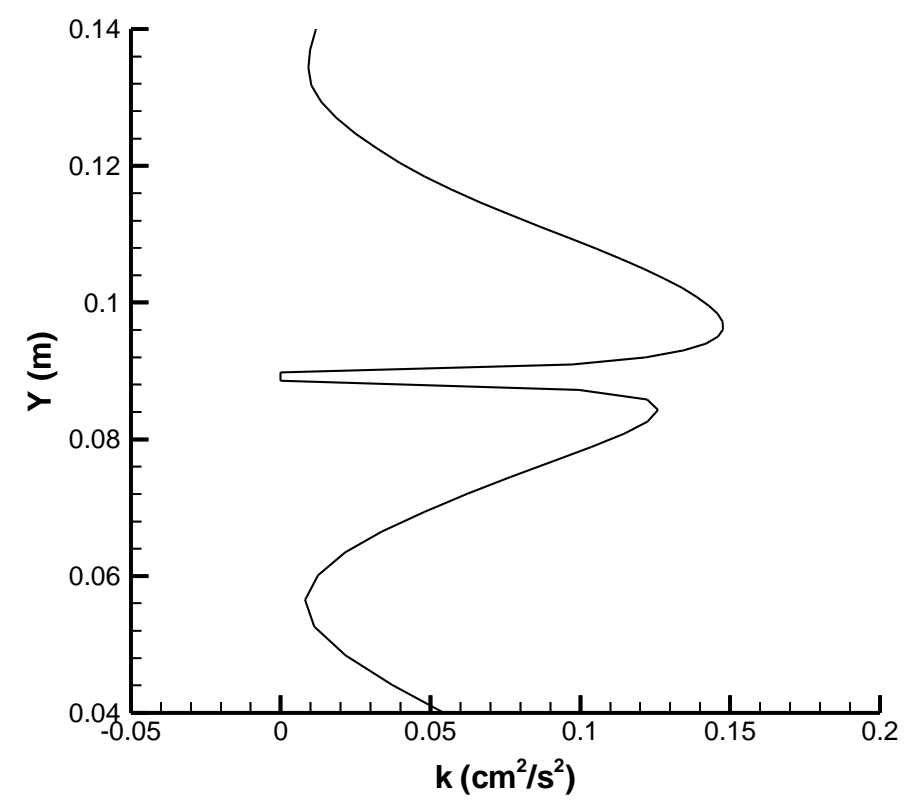

Figure 5.17 - Turbulent kinetic energy profile for case 13.

Considering the profiles that are predicted for $k$ and $\varepsilon$, the inflections or kinks that are evident in the velocity and volume fraction profiles can be related to the eddy viscosity and eddy diffusivity. Both the eddy viscosity and diffusivity are proportional to $k^{2} / \varepsilon$, which is plotted in Figure 5.18 for case 13 and shows the sharp change in this quantity at the interface. In light of the sudden change in the apparent viscosity at the interface, the velocity profile develops a large velocity gradient where the viscosity is low and then immediately develops a small velocity gradient where the viscosity is high. This maintains the continuity of the shear stress. A similar explanation can be given for the shape of the volume fraction profiles for the cases involving higher stratification. Like the profile for $k$, the increase in $\mathrm{k}^{2} / \varepsilon$ at the bottom of the plot in Figure 5.18 is due to the turbulence generated at the rough wall. 


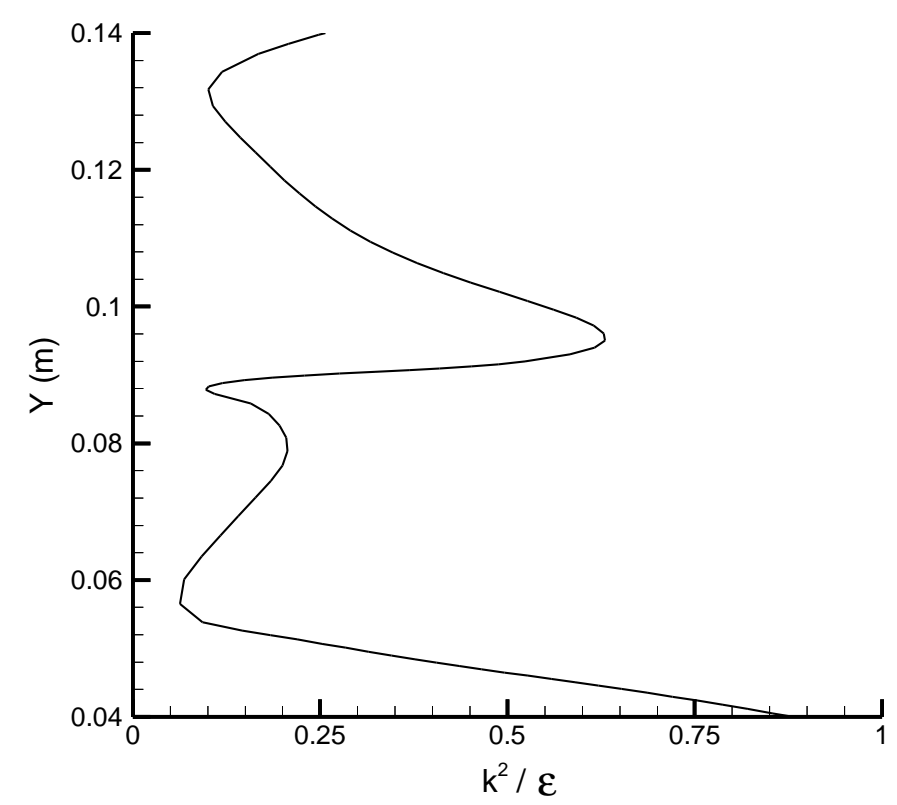

Figure 5.18 - Trend in eddy viscosity and eddy diffusivity for case 13.

What is needed in the model is some modification for low Reynolds number effects that occur near the interface. It would be desirably to multiply $\mu_{\mathrm{T}}$ by some function that would make the eddy viscosity approach zero near the interface. This is similar to the Van Driest modification to the mixing length near a solid boundary. A similar modification is usually made for the eddy viscosity in low Reynolds number $k$ - $\varepsilon$ models, where $\mu_{\mathrm{T}}$ is multiplied by some form of

$$
f_{\mu}=\left(1-\mathrm{e}^{-\mathrm{Re}_{\mathrm{T}}}\right)
$$

where $\mathrm{Re}_{\mathrm{T}}$ is a kind of turbulent Reynolds number. $\mathrm{Re}_{\mathrm{T}}$ is typically given by

$$
\operatorname{Re}_{\mathrm{T}}=\frac{\rho k^{2}}{\left(\varepsilon+\varepsilon_{\mathrm{O}}\right) \mu}
$$

where $\varepsilon_{O}$ is some constant value of $\varepsilon$ at the interface. Simulations using a form of the $k-\varepsilon$ model that included low Reynolds number corrections were not performed due to time 
constraints and due to the complexity associated with implementing the corrections in CFX. The simulations involving a low Reynolds number model would have involved coding the low Reynolds number corrections to the buoyancy source terms in a manner that is consistent with the CFX implementation of the low Reynolds number $k-\varepsilon$ model. The work that was anticipated to be involved with performing these implementations was out of the scope of the project.

Despite the disparity at the interface, the model does give reasonable predictions and trends for several of the interfacial parameters. In general the longitudinal averaging procedure tended to smooth out any irregularities in the data caused by the sudden change in the turbulence quantities. Hence, the longitudinally averaged values are presented instead of the values taken at the individual $\mathrm{x}$-locations, to show that the model captures many of the characteristics of a stratified shear flow with respect to the mean variables.

The predicted trend for the average interfacial thickness, $\delta$, was good for all four cases, though the predicted magnitude was somewhat higher than the experimental values. For increasing density stratification, the interfacial thickness generally decreases according to some power law of the Richardson number.

The quantity $\delta / \mathrm{H}$ is plotted against the overall Richardson number in Figure 5.19 using the experimental data and using the predicted values from the medium and fine grids. Since the total depth of both layers, $\mathrm{H}$, does give some characteristic length scale common to both the experiments and simulations, it was used to normalize $\delta$. The overall 
Richardson number is used here since it is the same for all four cases as defined by Eq. (5.1.12). While their is some question in fitting power laws to relatively few data points, to demonstrate the consistency of the present model with the experiments, a power law curve has also been fitted to each of the three sets of points. The power law as given by the simulations over the four data points is approximately $\delta / \mathrm{H} \sim\left(\mathrm{Ri}^{*}\right)^{-2.1}$ in Figure 5.19. The power law curve that was fit to the experimental data is given by $\delta / H \sim\left(\mathrm{Ri}^{*}\right)^{-2.6}$ in Figure 5.19.

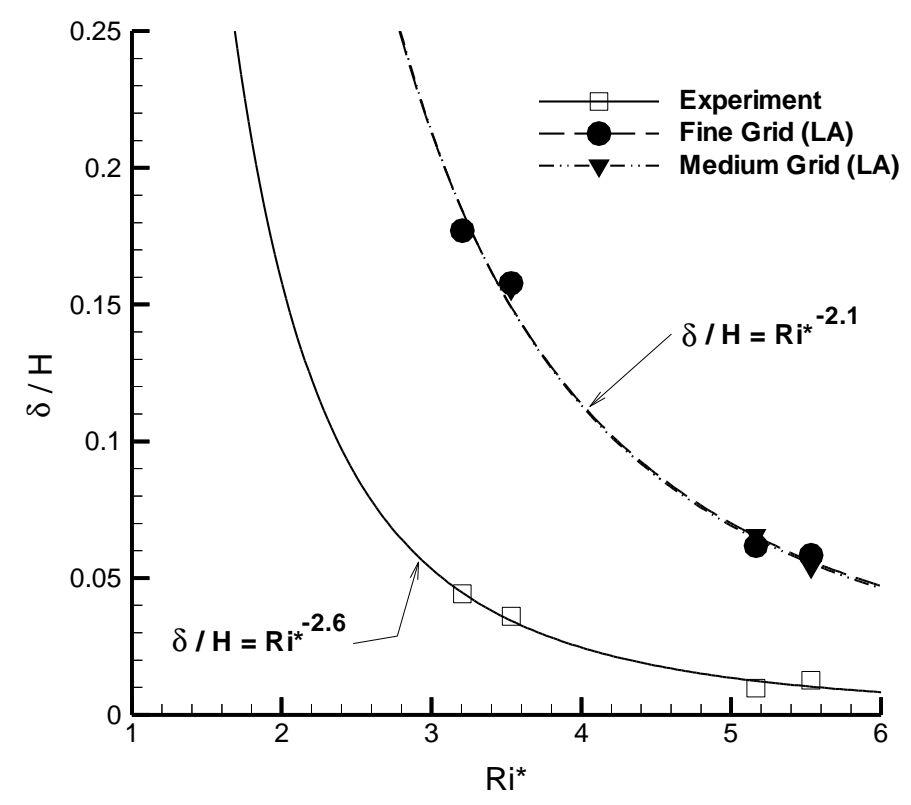

Figure 5.19 - Normalized interfacial thickness versus Ri*.

The degree to which $\delta$ will decrease is proportional to the turbulence in the flow and the buoyancy, therefore how $\delta$ decreases should be normalized by some direct measure of the turbulence. In the experiments Sullivan and List plotted $\delta / h_{S}$ versus Ris. This gives a 
measure of the interface thickness, normalized by the amount of shear or turbulence in the flow. In the simulations the experimentally predicted trend in $\mathrm{h}_{\mathrm{S}}$ was not well predicted as it is a parameter that is directly influenced by the velocity at the interface, which exhibited unphysical behavior given the state of the turbulence quantities at the interface. Hence, $\mathrm{h}_{\mathrm{S}}$ and $\mathrm{Ri}_{\mathrm{S}}$ were not good parameters to use for comparison with experimental trends; however, the magnitude of $\mathrm{Ri}_{\mathrm{S}}$ was consistently predicted close to the experimental values. Figure 5.20 shows $\mathrm{Ri}_{\mathrm{S}}$ plotted versus $\mathrm{Ri}^{*}$ from each of the four cases and demonstrates this agreement.

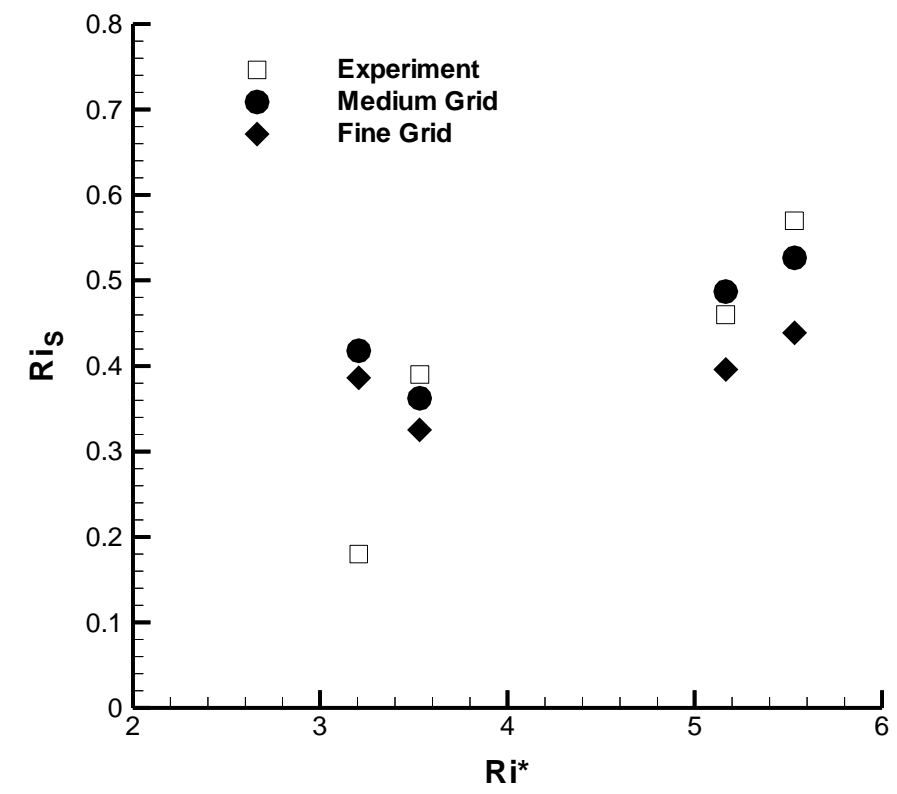

Figure 5.20 - Shear Richardson number versus Ri*.

In considering the overall performance of the models, SFST1 and the $k$ - $\varepsilon$ model with the buoyancy production and dissipation terms gave reasonable predictions for the majority of the relevant parameters. The interfacial parameters were the most difficult to predict, 
presumably because of low Reynolds number effects that are not accounted for by the present form of the $k-\varepsilon$ model. All other quantities, when considered in light of the longitudinal averages, were well predicted in both trend and magnitude. 


\subsection{SIMULATION OF STRATIFIED, SHEARING FLOW OF IMMISCIBLE FLUIDS}

Steady state shearing flows involving diesel fuel and fresh water flowing in a laboratory channel apparatus were simulated using CFX 4.1. The simulations were based on a shear flow facility currently being used at Johns Hopkins University in Baltimore, Maryland under the direction of Dr. Joe Katz [20]. Quantitative data from these experiments was unavailable; however, some comparison between the experimental flow field and the simulations was made. This comparison was based on qualitative images of the flow field that were observed during an experimental test at Johns Hopkins [20].

Altogether, four cases were simulated where the overall Richardson number and the average droplet size, which was assumed to be constant in each case, were varied. Time averaged, steady state solutions were obtained using SFST2, which is appropriate for flows involving immiscible fluids, and the $k-\varepsilon$ turbulence model with the buoyancy source terms. Of primary interest in these simulations was the trend in the gradient Richardson number over the vertical shear layer and along the streamwise direction. The gradient Richardson number is a local parameter and can be helpful in describing local effects in the flow field. The effects of the slip velocity due to fuel droplets rising through the water and water droplets falling through the fuel was also investigated. Trends for the interface thickness as functions of the maximum gradient Richardson number were studied as well. 


\subsection{Experimental Conditions Simulated}

The experimental setup consisted of essentially two stratified, immiscible, fluid layers flowing opposite to each other in a laboratory, channel apparatus. The apparatus, as shown in Figure 6.1, was approximately $1.5 \mathrm{~m}$ long, $32.0 \mathrm{~cm}$ high, and $7.5 \mathrm{~cm}$ wide. The flow was essentially two dimensional, but there could have been some three dimensional effects due to side walls with a large aspect ratio of $\mathrm{H} / \mathrm{w}=32 / 7.5$. The water and fuel inlets were both $11.0 \mathrm{~cm}$ high. In the experiments, fuel with a density of $850.0 \mathrm{~kg} / \mathrm{m}^{3}$, entered the apparatus from the upper left, while water, with a density of $1000.0 \mathrm{~kg} / \mathrm{m}^{3}$, entered from the lower right at a significantly higher flow rate than the fuel. An inlet diffuser smoothly transitioned the fuel into the oncoming water, where the shear develops. Some of the fuel exited the domain through the top outlet into a fuel reservoir, while most of the fuel, which was entrained in the water, exited through the lower outlet. An outlet weir below the fuel inlet diffuser acted to direct the flow of water along the fuel interface.

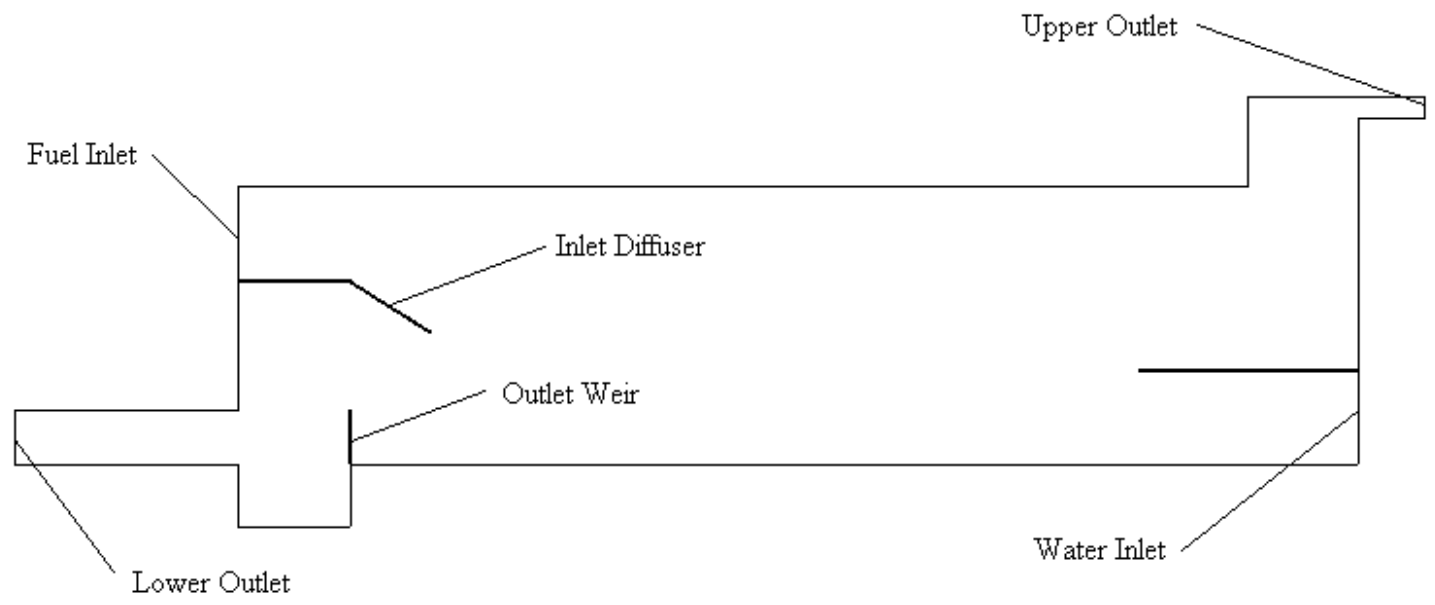

Figure 6.1 - Johns Hopkins shear flow experimental setup. 
The main shear region between the fuel-inlet diffuser and the water-inlet splitter plate was approximately $0.8 \mathrm{~m}$ in length. Typical inlet flow rates for the fuel were approximately $0.00095 \mathrm{~m}^{3} / \mathrm{s}$ corresponding to a mean inlet velocity of $0.10 \mathrm{~m} / \mathrm{s}$. Inlet flow rates for the water ranged from $0.0056 \mathrm{~m}^{3} / \mathrm{s}$ to $0.0075 \mathrm{~m}^{3} / \mathrm{s}$ corresponding to mean inlet velocities of $0.68 \mathrm{~m} / \mathrm{s}$ to $0.92 \mathrm{~m} / \mathrm{s}$. The same definitions as were used for the Sullivan and List simulations for the overall Richardson numbers, mixed layer thickness, and interfacial thickness were used in describing the flow. The overall Richardson number as given by Eq. 5.1.12 was defined in relation to inlet velocities and densities and was used to characterize different cases. The relative effects of shear and buoyancy were characterized using the gradient Richardson number given locally in the flow field by

$$
\operatorname{Ri}_{\mathrm{g}}=\frac{-(\mathrm{g} / \rho)(\partial \rho / \partial \mathrm{y})}{(\partial \mathrm{u} / \partial \mathrm{y})^{2}}
$$

This parameter was used because the flow field that is generated in the shear flow apparatus as is shown in Figure 6.1 was not homogeneous in the longitudinal direction. Therefore, characterizing the state of the flow by a single Richardson number was difficult and hence a local parameter was used. A schematic of the complete shear flow facility is given in Figure 6.2 as provided by Katz [20]. 


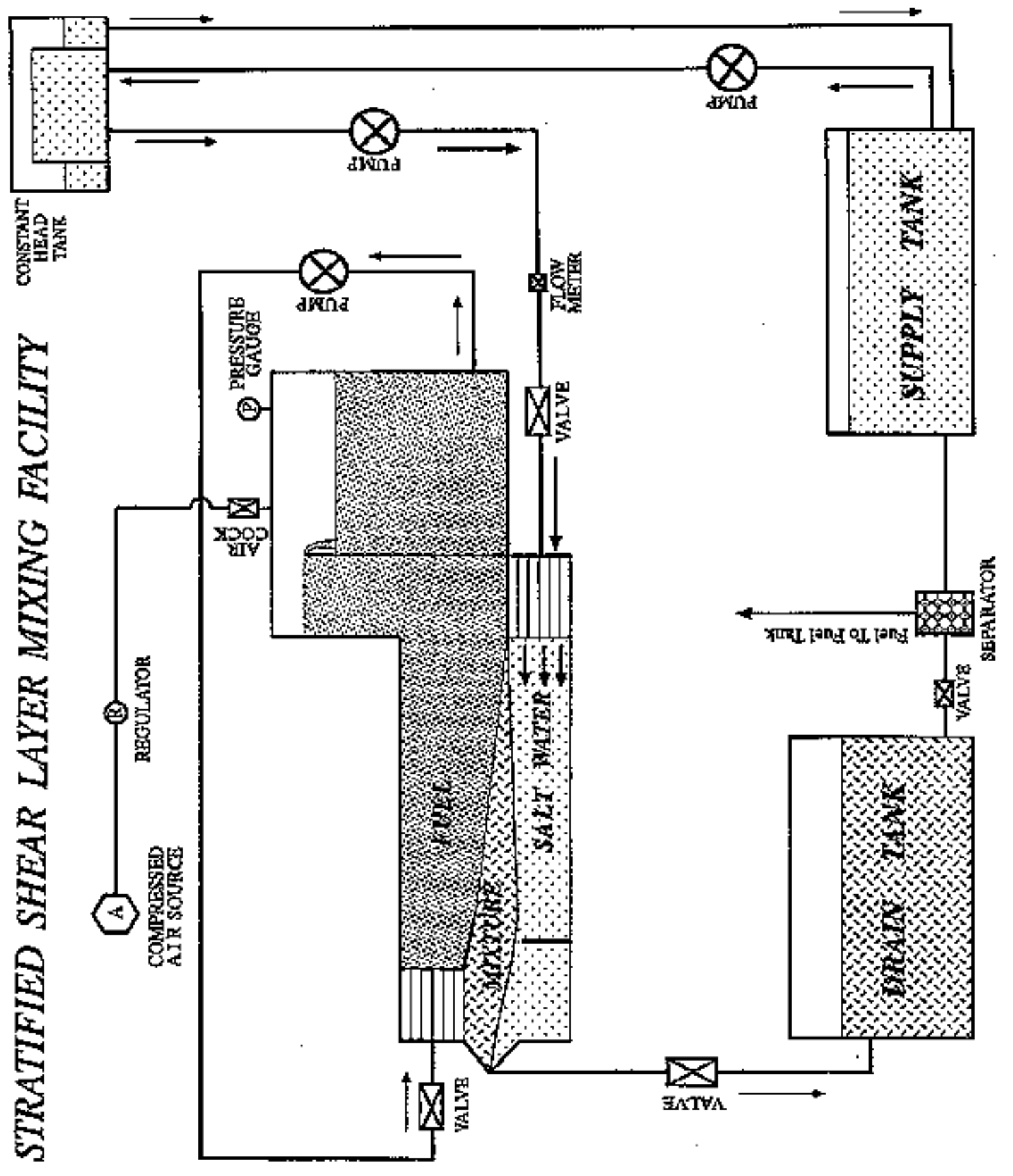

Figure 6.2 - Johns Hopkins shear flow facility; After Katz 1998 [20]. 


\subsection{Computational Details}

In light of the experimental scenario and governing parameters, a two-dimensional model was configured in CFX 4.1. SFST2, was used to model the mixing of the two immiscible fluids. In the formulation of the SFST model that was used, the mixture definitions, momentum equations, and mass conservation equations as presented in Section 3.2 were adopted as the governing equations. The $k$ - $\varepsilon$ model with the buoyancy source terms, as described in Section 4.3, was used to model the effects of turbulence in these simulations.

The constitutive equation for the relative motion between the phases in the vertical direction was assumed to be predominately given by the terminal rise velocity of a single fluid droplet. This relationship assumes that a balance exists between drag, gravity, and buoyancy forces on a fluid droplet. The resulting equation is given by

$$
u_{\mathrm{s}}=\left(\frac{\mathrm{g} 4 \mathrm{~d}_{\mathrm{p}}}{3 \mathrm{C}_{\mathrm{D}}}\right)^{1 / 2}\left(\frac{\rho_{\beta}-\rho_{\alpha}}{\rho_{\beta}}\right)^{1 / 2}
$$

where $u_{S}$ is the slip velocity, $\alpha$ denotes the dispersed phase and $\beta$ denotes the continuous phase. The derivation of Eq. (6.2.1) is given in Appendix A.6. Two parameters of interest that will influence the magnitude of the slip velocity are the particle drag coefficient, $C_{D}$, and the size of the average droplet diameter, $d_{p}$. In the simulations $d_{p}$ was used as a parameter that was set equal to a constant. In reality, depending on the local flow field and the fluid properties, droplets of different sizes will be formed. In the simulations such events were not modeled and an average droplet size was assumed. This allowed the effects of the average droplet size on the flow field to be investigated. 
The attention now will be turned to the drag coefficient, which is defined by

$$
C_{D}=\frac{\text { DragForce }}{\rho_{\beta} d_{p}^{2} u_{S}^{2}(8 / \pi)}
$$

in the following discussion. The laws governing the coefficient of drag for a fluid droplet can be complicated by the variation in drag regimes and the possible distortion of the droplet shape. For cases where the distortion of the droplet is negligible, the drag laws in a fluid droplet system may be approximated by the same drag laws that are used in a solid particle system [18]. The drag coefficient is usually given as a function of the particle Reynolds number, which is defined by

$$
\operatorname{Re}_{\mathrm{p}}=\frac{\rho_{\beta} \mathrm{u}_{\mathrm{s}} \mathrm{d}_{\mathrm{p}}}{\mu_{\beta}}
$$

for a droplet that is moving in a continuous fluid denoted by $\beta$. The subscript $\alpha$ represents the disperse phase in the present discussion.

Initially the drag is totally due to viscous influences. This is known as Stokes' regime, which is typically defined for particle Reynolds numbers less than 1 . Next the droplet goes through a transitional region where the drag is due to viscous and inertial influences in what is typically referred to as Allen's regime. Allen's regime is typically defined for particle Reynolds numbers in the region between 1 and 1,000. In the third regime, which is referred to as Newton's regime, the drag is due almost entirely to inertial forces and the drag coefficient is approximately a constant. Newton's regime is usually defined for particle Reynolds numbers in the region between 1,000 and 100,000. Finally, at some 
critical particle Reynolds number, the boundary layer transitions from laminar to turbulent. Since the boundary layer remains attached further on the back face of the droplet this causes drag reduction, and the coefficient of drag is again approximately a constant. This regime is typically referred to as the super-critical regime, and is approximately the regime defined for particle Reynolds number that are greater than $1,000,000$.

In all of the simulations the magnitude of the droplet Reynolds numbers were in the low end of Allen's regime. In Allen's regime several correlations are available for calculating the coefficient of drag. Ishii [18] gives one correlation for calculating the drag coefficient in Allen's regime by

$$
C_{D}=\frac{24}{\operatorname{Re}_{p}}\left(1+0.1 \operatorname{Re}_{p}^{0.75}\right)
$$

which was found to be widely used in the literature. Computationally, using Eq. (6.2.4) requires iteration since the exact value of $\mathrm{Re}_{\mathrm{P}}$ is unknown if the value of the slip velocity is unknown. Using an approximate power law relationship for $\mathrm{C}_{\mathrm{D}}$ allows the coefficient of drag to be absorbed into the slip velocity relationship and removes the need for iteration. This is shown in Appendix A.6. An approximate power law curve for Allen's regime, which closely follows the correlation given by Eq. (6.2.4), is given by

$$
\mathrm{C}_{\mathrm{D}}=\frac{18}{\operatorname{Re}_{\mathrm{P}}^{0.6}}
$$

Figure 6.3 compares the two correlations given by Eq. (6.2.4) and Eq. (6.2.5) where $C_{D}$ is plotted against $\mathrm{Re}_{\mathrm{P}}$ in $\log -\log$ coordinates. 


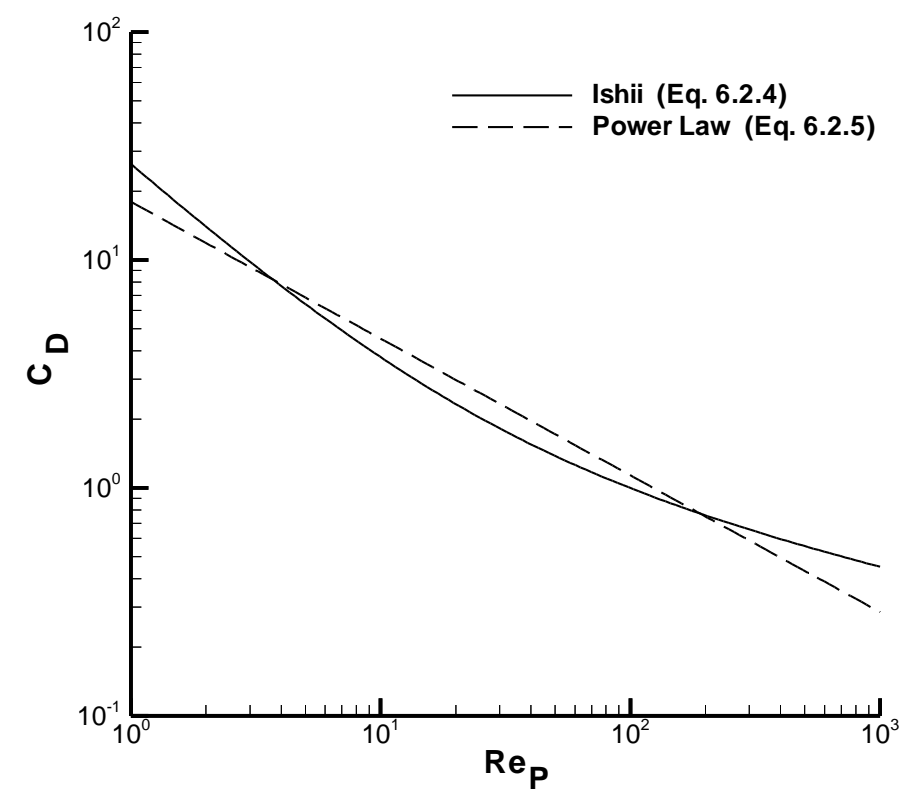

Figure 6.3 - Comparison of drag correlations in Allen's regime.

Using the relationship for $C_{D}$ that is given by Eq. (6.2.5) in Eq. (6.2.1) gives the slip velocity equation by

$$
\mathrm{u}_{\mathrm{S}}=\left\{\left(\frac{4 \mathrm{gd}_{\mathrm{p}}^{1.6}}{54 \mu_{\beta}^{0.6}}\right)\left(\frac{\rho_{\beta}-\rho_{\alpha}}{\rho_{\beta}^{0.4}}\right)\right\}^{5 / 7}
$$

as is shown in Appendix A.6. This relationship was assumed to be valid over the range of particle Reynolds numbers between 1 and 1,000, and it was used in all of the simulations for the shear flow experiments involving immiscible fluids. Particle Reynolds numbers outside the anticipated range did not occur in any of the simulations. 
One important assumption that is made when a slip velocity relationship similar to Eq. (6.2.1) is used is that that multiple particle effects are ignored. The presence of multiple particles or droplets will tend to decrease the terminal rise velocity of a single droplet. Multiple particle effects can be accounted for by using an increased viscosity in the particle Reynolds number [18]. An increasing number of droplets will tend to decrease the Reynolds number and hence $C_{D}$ will increase. These effects are recognized to exist in the flow situations being considered; however, they are neglected for brevity in the present model.

Another important assumption that is inherent in using Eq. (6.2.1) is that the fluid droplets reach their terminal velocity very quickly. This amounts to assuming that the time interval between when the droplet is formed and when the droplet attains its terminal velocity given by Eq. (6.2.1) is very small. For Stokes' flow, the transient form of Eq. (6.2.1) can be obtained analytically and gives the time constant (relaxation time) for the particle by

$$
\tau_{\mathrm{dp}}=\frac{4 \rho_{\alpha} d_{p}^{2}}{72 \mu_{\beta}}
$$

where $\tau_{\mathrm{dp}}$ is the time it will take the particle to reach $63 \%$ of its terminal velocity. Eq. (6.2.7) was used as an estimate for the time constant of the average droplet sizes that were used in the simulations. For droplet sizes on the order of $2.5 \mathrm{~mm}$, typical values for $\tau_{\mathrm{dp}}$ are less than 0.2 seconds. When compared with the time scales of the mean flow for the 
simulations, this gives a measure of justification to the assumption that the fluid droplets quickly attain their terminal velocity.

Finally, it should be mentioned that effects of particle distortion are also neglected in the current formulation for the terminal rise velocity. As the particle Reynolds number increases, depending on the Weber-number reflecting surface tension effects, the fluid droplets may become ellipsoidal in shape and eventually form a spherical cap shape [2]. These effects tend to influence the drag coefficient and the terminal velocity of the droplet.

In regards to other relative velocity effects, it was assumed that the relative motion between the phases was essentially zero in the horizontal plane. With these assumptions the extra terms (i.e. the drift flux terms involving the product of the relative velocities) in Eq. (3.2.5) reduce to a single term in the vertical momentum equation. By computational experiments it was found that this single term can usually be neglected for flows, where the flow field is not induced by buoyancy and most of the inertial forces come from the mixture velocities. Hence the momentum equations that were solved for the present simulations were given by

$$
\frac{\partial\left(\rho_{m} u_{m, i}\right)}{\partial t}+\frac{\partial}{\partial x_{j}}\left(\rho_{m} u_{m, i} u_{m, j}\right)=-\frac{\partial P}{\partial x_{i}}+\frac{\partial}{\partial x_{j}}\left(\left(\mu_{m}+\mu_{T}\right)\left(\frac{\partial u_{m, i}}{\partial x_{j}}+\frac{\partial u_{m, j}}{\partial x_{i}}\right)\right)+\rho_{m} g_{i}
$$

and the primary influence of the slip velocity comes into the model through the volume fraction equation given by Eq. (3.2.9). 
To simulate the experimental setup, a 19 block geometry was used as is shown in Figure 6.4. Here the inlets, outlets, and the inner walls are shown as they were modeled in CFX; lines inside the geometry denote inter block boundaries as are used by the CFX solver. The dimensions of this model followed those that were used in the experiments.

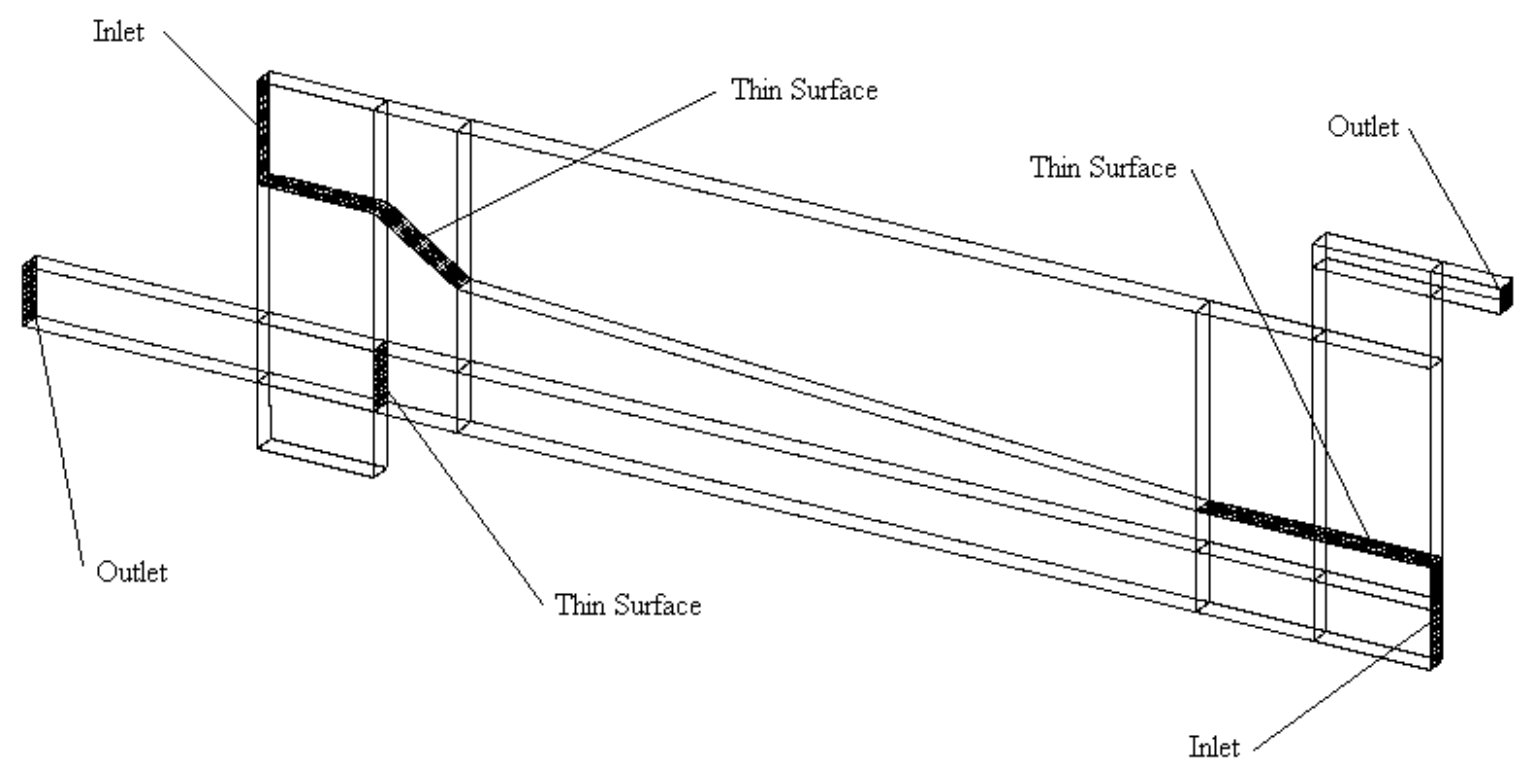

Figure 6.4 - Geometry used in CFX to simulate Johns Hopkins shear flow.

A fine grid consisting of 29,175 cells, was used for the simulations. In the main shear region, between the splitter plates, the fine grid consisted of 150 cells in the longitudinal direction by 104 cells in the vertical direction. Here the cells were concentrated towards the lower wall and vertical center of the model using a geometric progression factor of 1.02. This gave the smallest cell at or near the interface dimensions of approximately 5.0 $\mathrm{mm}$ long by $1.0 \mathrm{~mm}$ high. 
Boundary conditions for the inlets were modeled by setting a fixed velocity such that the inlet flow rates were the same as those in the experiments. Specified values of the volume fractions corresponding to the pure unmixed fluids were also set at the inlets. Values of $k$ and $\varepsilon$ were estimated at the inlets using Eq. (5.2.1) and Eq. (5.2.2) and pressure at the inlets was extrapolated from downstream. Hence Dirichlet boundary conditions were specified on all quantities except pressure at the inlet.

Boundary conditions at the outlets was set as if the fluid was exiting the domain with a free surface at the top of the outlet boundary in the longitudinal direction. This boundary condition corresponds to the same boundary condition described in Section 5.2. In addition to the hydrostatic distribution a constant pressure of 3,448.0 $\mathrm{Pa}$ was set at the upper right outlet, corresponding to a pressure difference of 0.5 psi between the top and bottom outlets. This was done since the fuel outlet tank on the right of the apparatus is typically pressurized in the experiments. Boundary conditions for all other quantities at the outlets where modeled by setting a zero derivative condition.

Four cases were simulated involving variations in the overall Richardson number and estimated droplets size, $d_{p}$, that is used in Eq (6.2.1). Quantities as set at the inlets for each case are listed in Table 6.1. 
Table 6.1 - Inlet Flow Parameters and Overall Parameters for CFX model

\begin{tabular}{ccccccc}
\multicolumn{2}{c}{ Lower inlet parameters } & & & & \multicolumn{2}{c}{ Overall Parameters } \\
Case & $\mathrm{u}(\mathrm{cm} / \mathrm{s})$ & $\rho\left(\mathrm{kg} / \mathrm{m}^{3}\right)$ & $k\left(\mathrm{~cm}^{2} / \mathrm{s}^{2}\right)$ & $\varepsilon\left(\mathrm{cm}^{2} / \mathrm{s}^{3}\right)$ & $\mathrm{d}_{\mathrm{p}}(\mathrm{mm})$ & $\mathrm{Ri}^{*}$ \\
1 & 69.03 & 1000.00 & 9.530 & 3.434 & 1.75 & 0.85 \\
2 & 69.03 & 1000.00 & 9.530 & 3.434 & 2.35 & 0.85 \\
3 & 92.04 & 1000.00 & 16.943 & 6.105 & 1.75 & 0.51 \\
4 & 92.04 & 1000.00 & 16.943 & 6.105 & 2.35 & 0.51
\end{tabular}

Upper Inlet parameters

$\begin{array}{ccccc}\text { Case } & \mathrm{u}(\mathrm{cm} / \mathrm{s}) & \rho\left(\mathrm{kg} / \mathrm{m}^{3}\right) & k\left(\mathrm{~cm}^{2} / \mathrm{s}^{2}\right) & \varepsilon\left(\mathrm{cm}^{2} / \mathrm{s}^{3}\right) \\ 1 & 11.50 & 850.00 & 0.265 & 0.095 \\ 2 & 11.50 & 850.00 & 0.265 & 0.095 \\ 3 & 11.50 & 850.00 & 0.265 & 0.095 \\ 4 & 11.50 & 850.00 & 0.265 & 0.095\end{array}$

The Hybrid scheme was used to discretise the velocity components, and the volume fraction equation was discretised using first order upwinding. Pressure was calculated using central differencing, while the turbulence quantities were calculated using Hybrid differencing.

All simulations were run using transient marching to steady state. Typically 50 outer iterations were performed over about 280 time steps of 0.1 second. This corresponded to approximately three flow-through times for the fuel and about 25 flow-through times for the water. At this time the outlet flow rates and vertical profiles of all the quantities remained constant and the solution was taken as the steady state. 


\subsection{Results and Discussion}

In discussing the predictions as given by the computational model, the overall flow field will be considered first, then the vertical profiles of the gradient Richardson number will be discussed, and finally the qualitative experimental comparisons will be considered. The gradient Richardson number profiles will give some idea how the local scales of buoyancy to turbulence change for this flow and will be discussed in light of the overall flow field parameters. The trend in the gradient Richardson number over the vertical shear layer and along the streamwise direction will be shown to influence the magnitude of the interfacial thickness, $\delta$, and the mixed layer thickness, $\delta_{\mathrm{M}}$.

Possible improvements to the multiphase and turbulence models will also be discussed, namely the influence of the slip velocity on the momentum equations, which was neglected in the simulations, and the influence of surface tension effects will be considered. Since the flow in this geometry is somewhat intricate, overall contours showing the volume fraction and the streamlines are shown for each case in Figure 6.6 through Figure 6.13. These contours give some indication as to how the overall Richardson number and the average droplet size affect the overall flow field and the interfacial thickness. The various obstacles associated with the geometry cause numerous zones of re-circulation to develop in the flow field, with one of the most noticeable recirculation zone occurring at the end of the fuel inlet diffuser. 
An important fact to keep in mind when examining the streamlines in Figures 6.6, 6.8, 6.10 , and 6.12 is that the fuel is moving quite slowly compared to the water. Essentially, the fuel is almost quiescent and the water causes the majority of the shear. To put some perspective on the velocity of the fuel and the water, Figure 6.5 shows velocity vectors and the fuel volume fraction for case 1 where the development of a stratified shear layer can be seen at the interface. The maximum velocity in Figure 6.5 is $70.0 \mathrm{~cm} / \mathrm{s}$ and occurs in the lower layer.

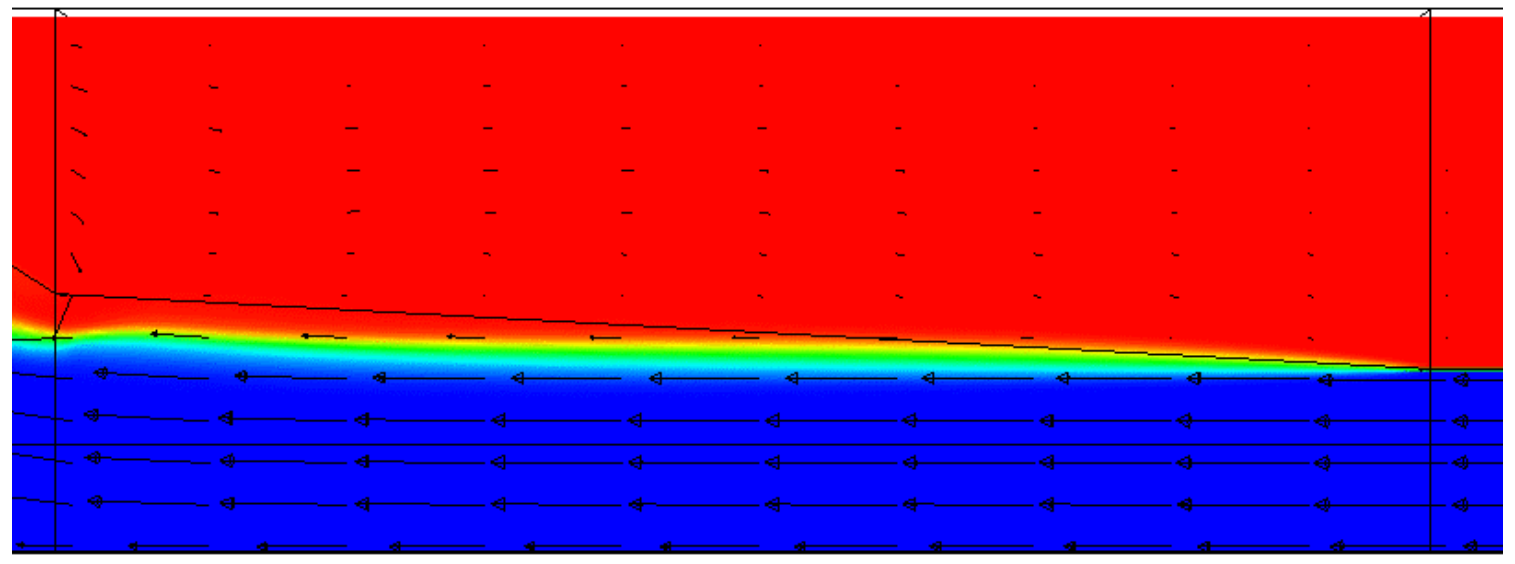

Volume Fraction
0.00
0.25
0.50
0.75
1.00

Figure 6.5 - Volume fraction contours and velocity vectors; $\mathrm{Ri}^{*}=0.85, \mathrm{~d}_{\mathrm{p}}=1.75 \mathrm{~mm}$. 

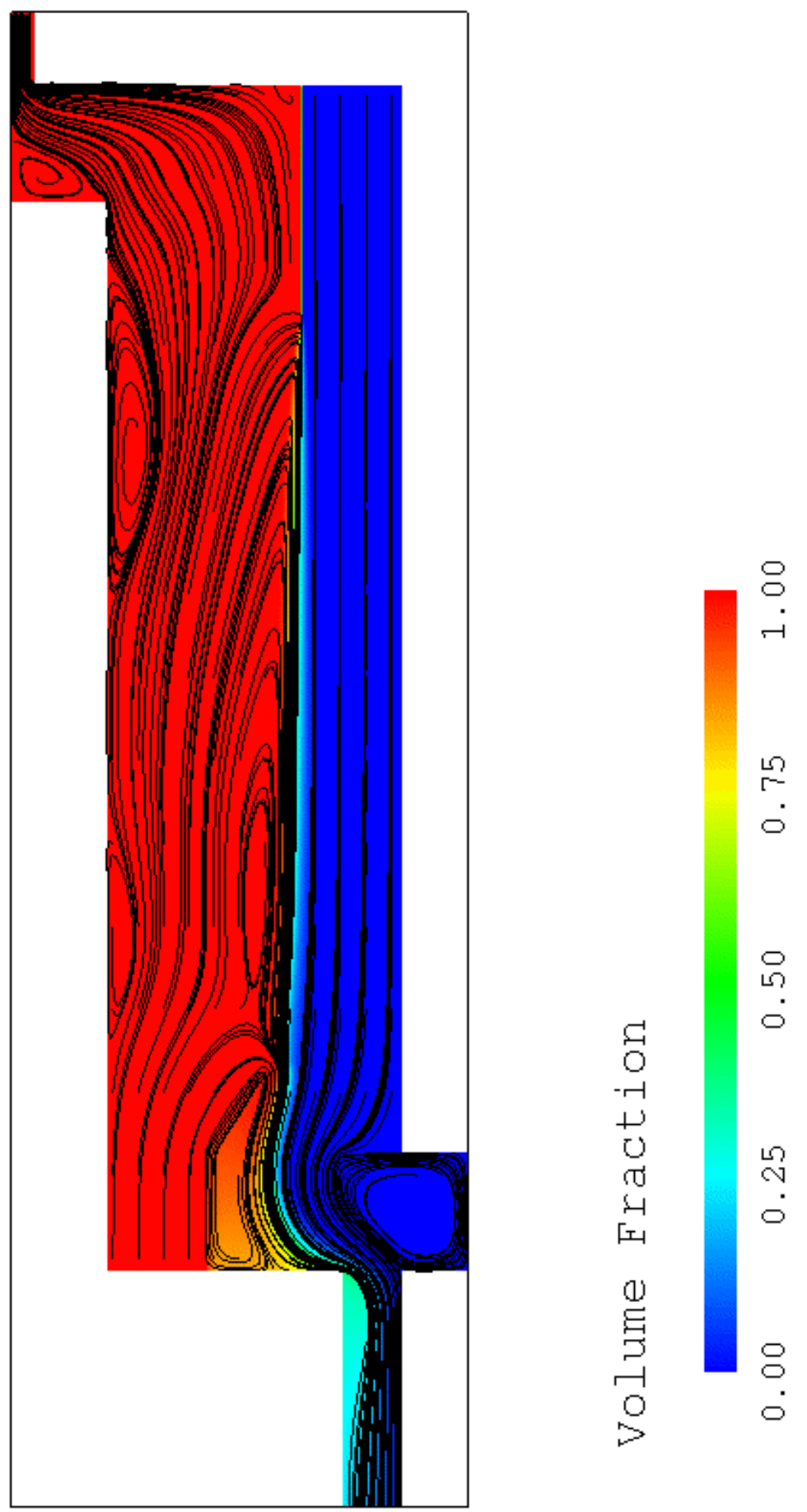

Figure 6.6 - Streamlines and volume fraction contours; $\mathrm{Ri}^{*}=0.85, \mathrm{~d}_{\mathrm{p}}=1.75 \mathrm{~mm}$. 


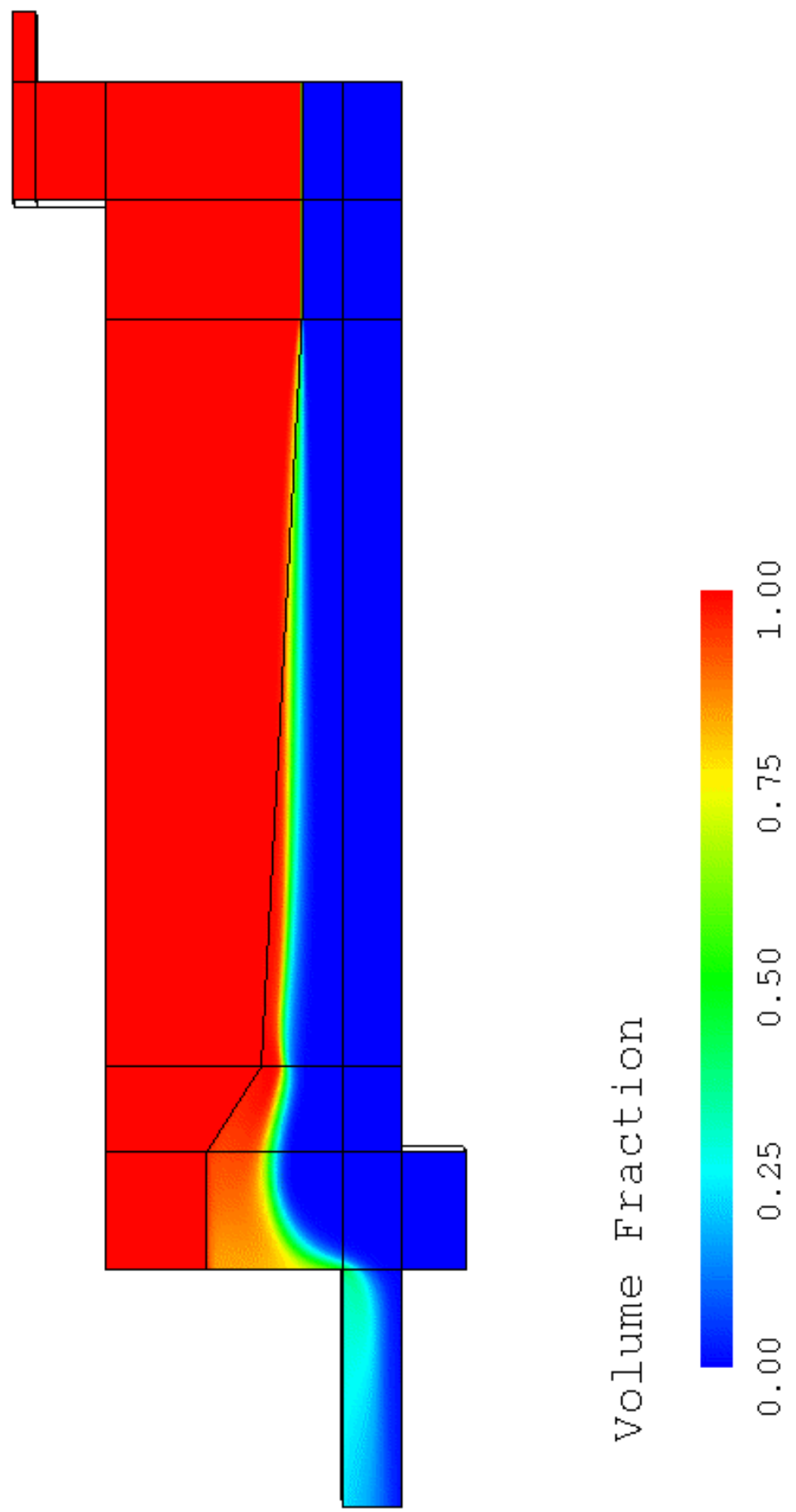

Figure 6.7 - Volume fraction contours; $\mathrm{Ri}^{*}=0.85, \mathrm{~d}_{\mathrm{p}}=1.75 \mathrm{~mm}$. 

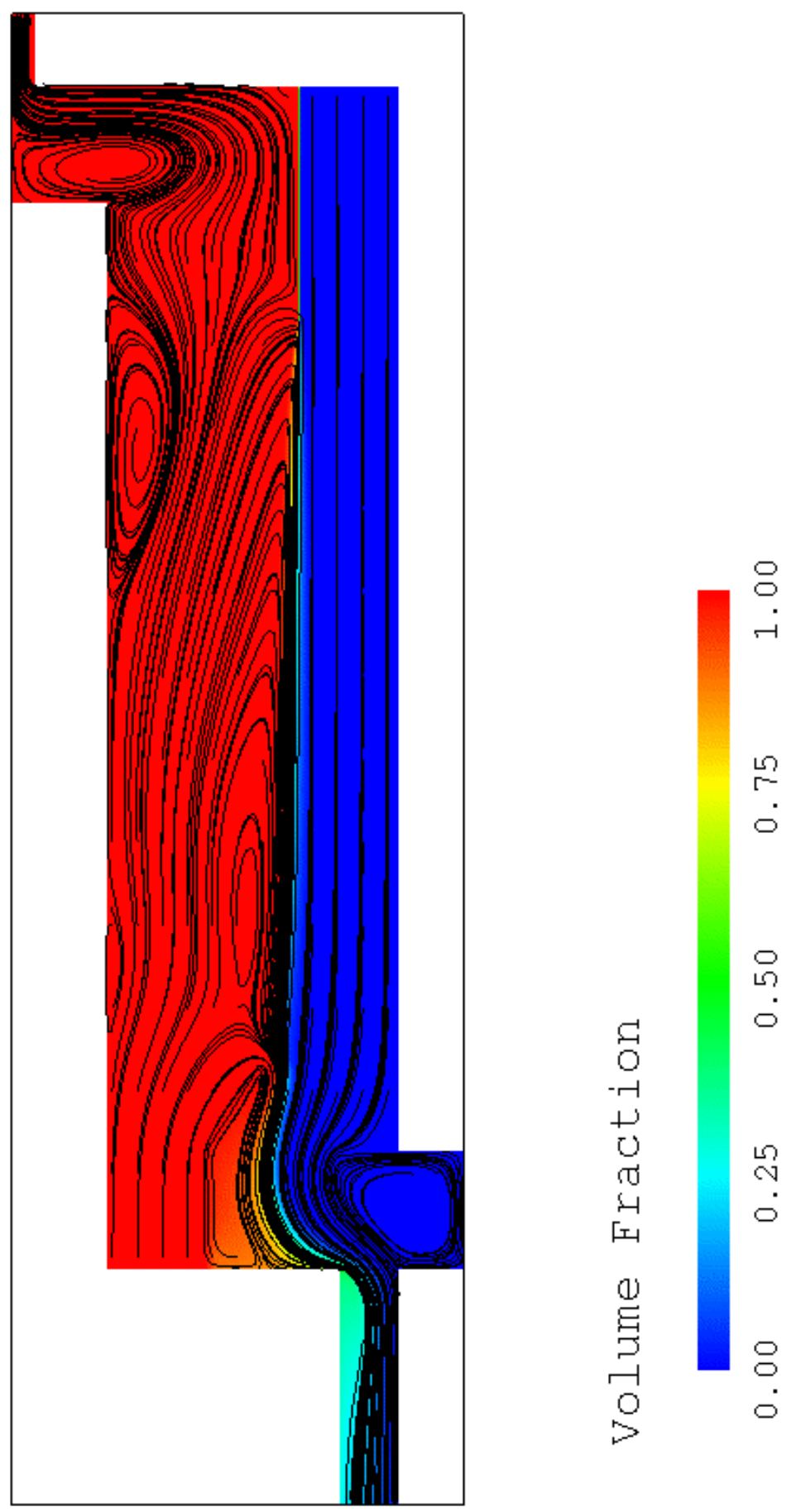

Figure 6.8 - Streamlines and volume fraction contours; $\mathrm{Ri}^{*}=0.85, \mathrm{~d}_{\mathrm{p}}=2.35 \mathrm{~mm}$. 

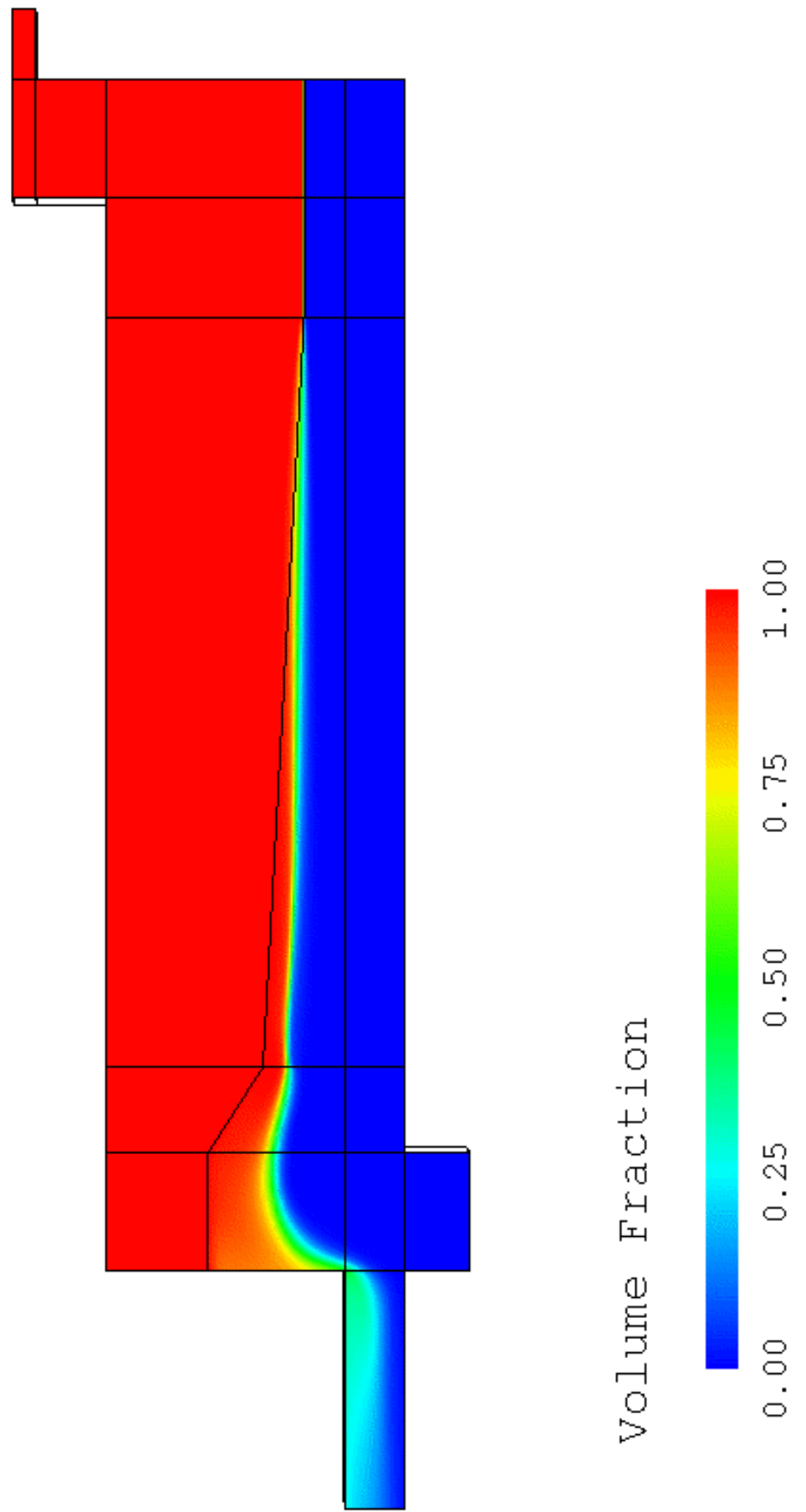

Figure 6.9 - Volume fraction contours; $\mathrm{Ri}^{*}=0.85, \mathrm{~d}_{\mathrm{p}}=2.35 \mathrm{~mm}$. 

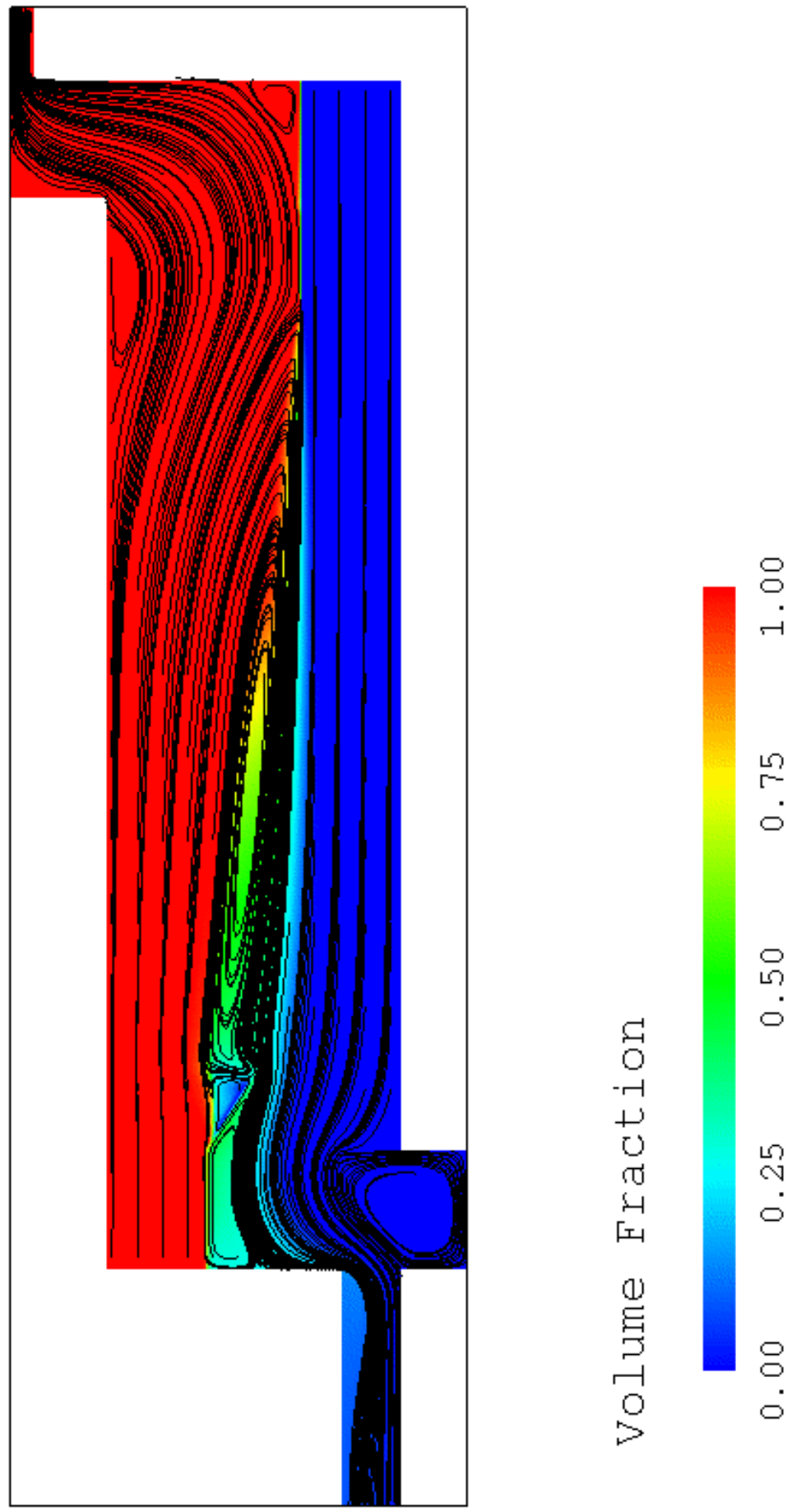

Figure 6.10 - Streamlines and volume fraction contours; $\mathrm{Ri}^{*}=0.51, \mathrm{~d}_{\mathrm{p}}=1.75 \mathrm{~mm}$. 


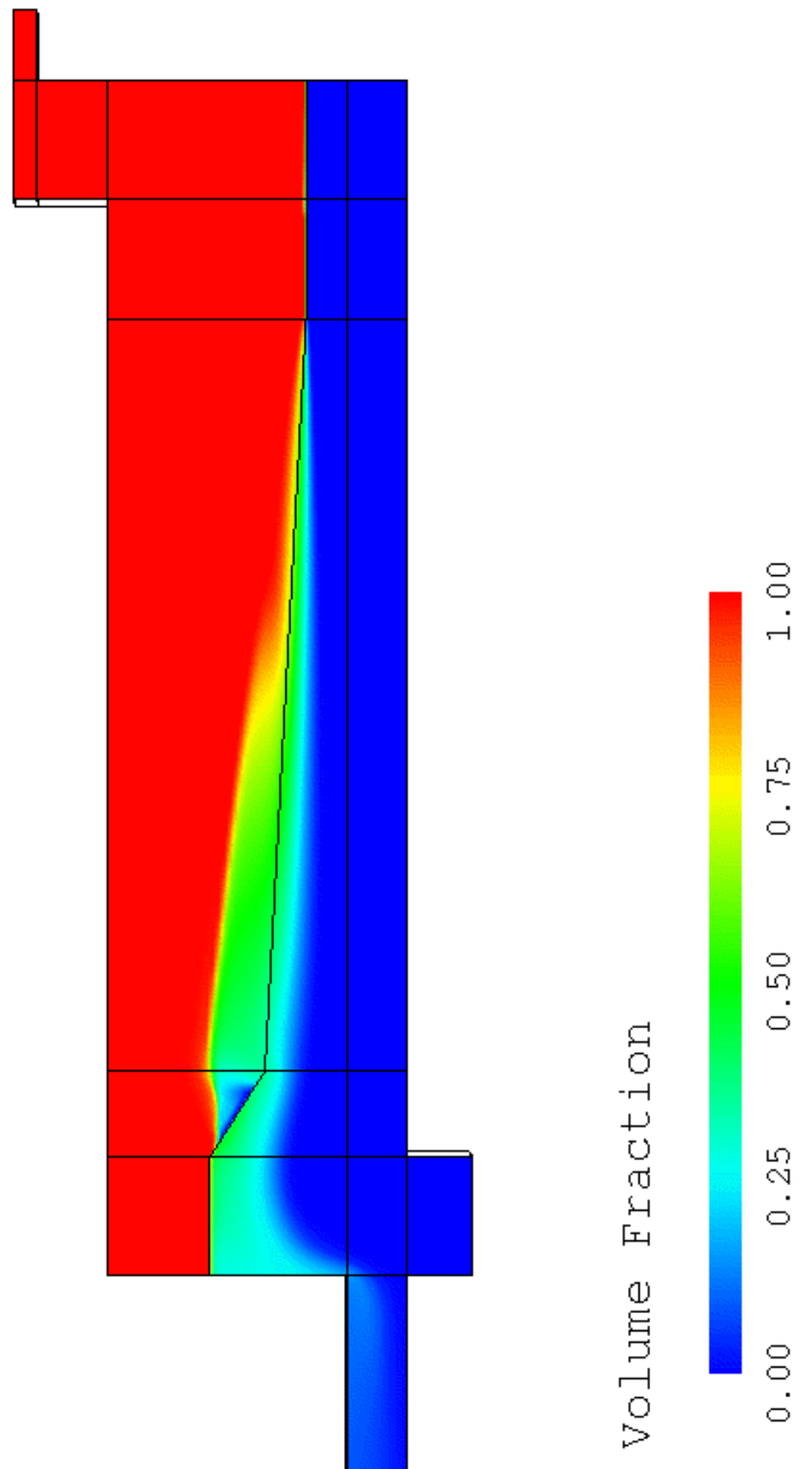

Figure 6.11 - Volume fraction contours; $\mathrm{Ri}^{*}=0.51, \mathrm{~d}_{\mathrm{p}}=1.75 \mathrm{~mm}$. 

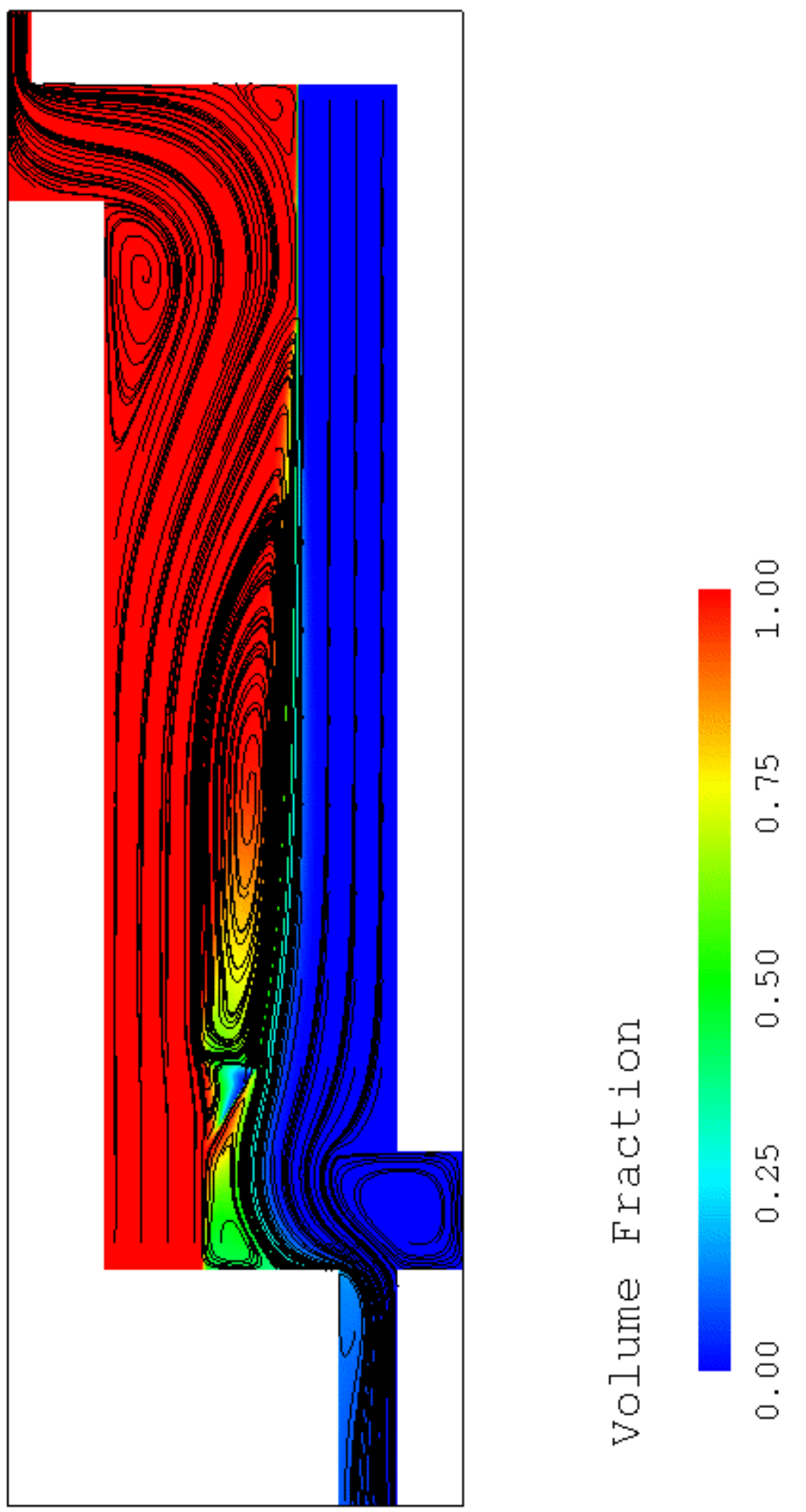

Figure 6.12 - Streamlines and volume fraction contours; $\mathrm{Ri}^{*}=0.51, \mathrm{~d}_{\mathrm{p}}=2.35 \mathrm{~mm}$. 

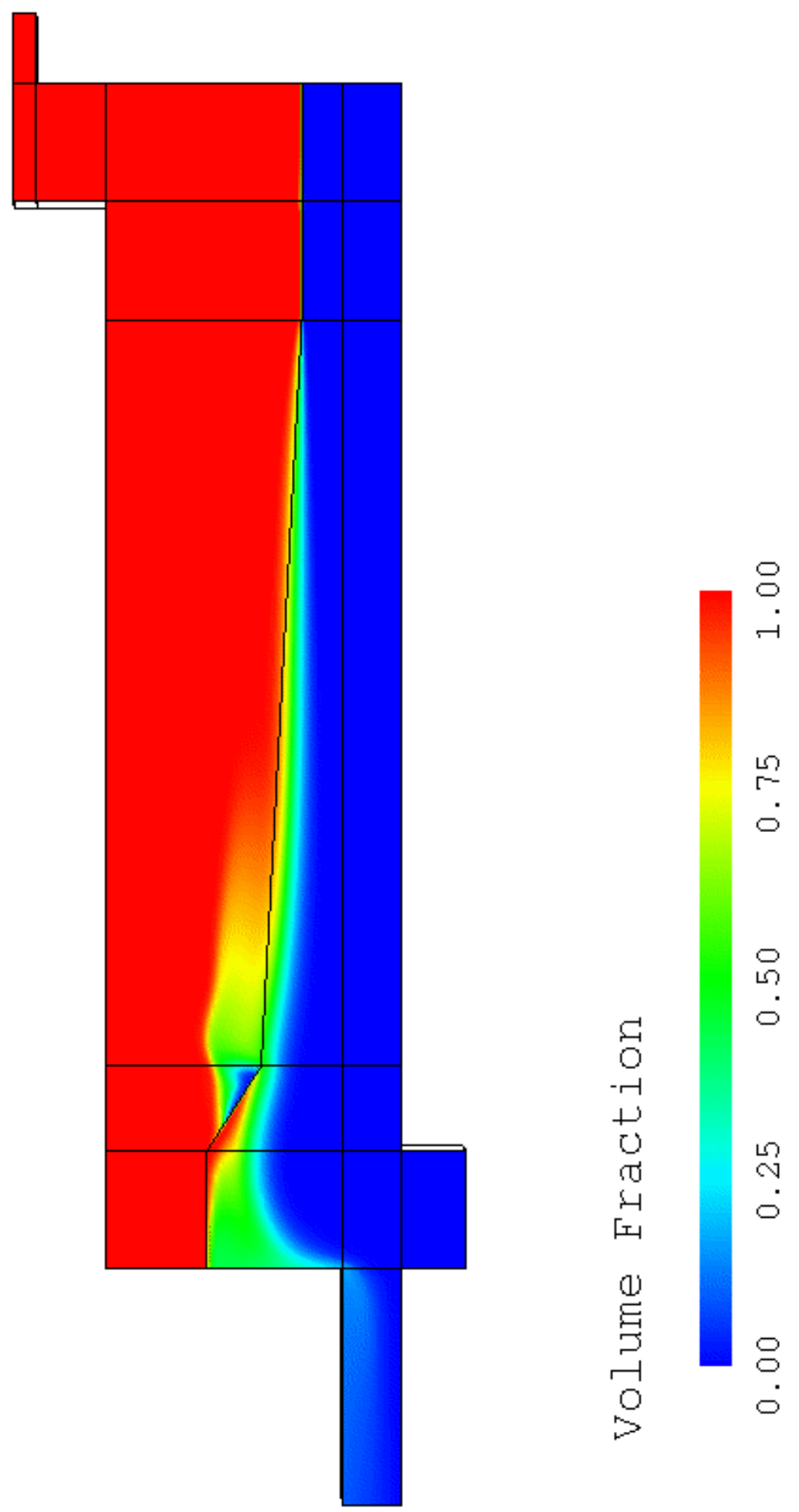

Figure 6.13 - Volume fraction contours; $\mathrm{Ri}^{*}=0.51, \mathrm{~d}_{\mathrm{p}}=2.35 \mathrm{~mm}$. 
One of the most obvious details about the flow field is the complexity associated with the numerous zones of re-circulation. This is evident in Figures 6.6, 6.8, 6.10, and 6.12, with at least 8 re-circulation zones appearing in Figure 6.12. Several of the features that are observed in the profiles of the shear flow from the simulations are also observed experimentally. Namely, the thickness of the mixed layer at the interface and the recirculation near the fuel inlet are experimentally observed.

Three instantaneous images of the sheared interface were obtained from the shear flow facility at Johns Hopkins [38] and are shown in Figure 6.14 through Figure 6.16. The flow rate that was used in the experiments was 120 GPM and corresponded to the inlet velocities that were used in the simulations for cases 3 and 4 . Hence, some comparison can be made between the experimental profiles and the profiles from the simulations that are given in Figures 6.10 through 6.13. It should be noted here that these comparisons are between time averaged quantities from the simulations and instantaneous experimental quantities. Hence, exact comparison is difficult; however, some measure of similarity can be made. 


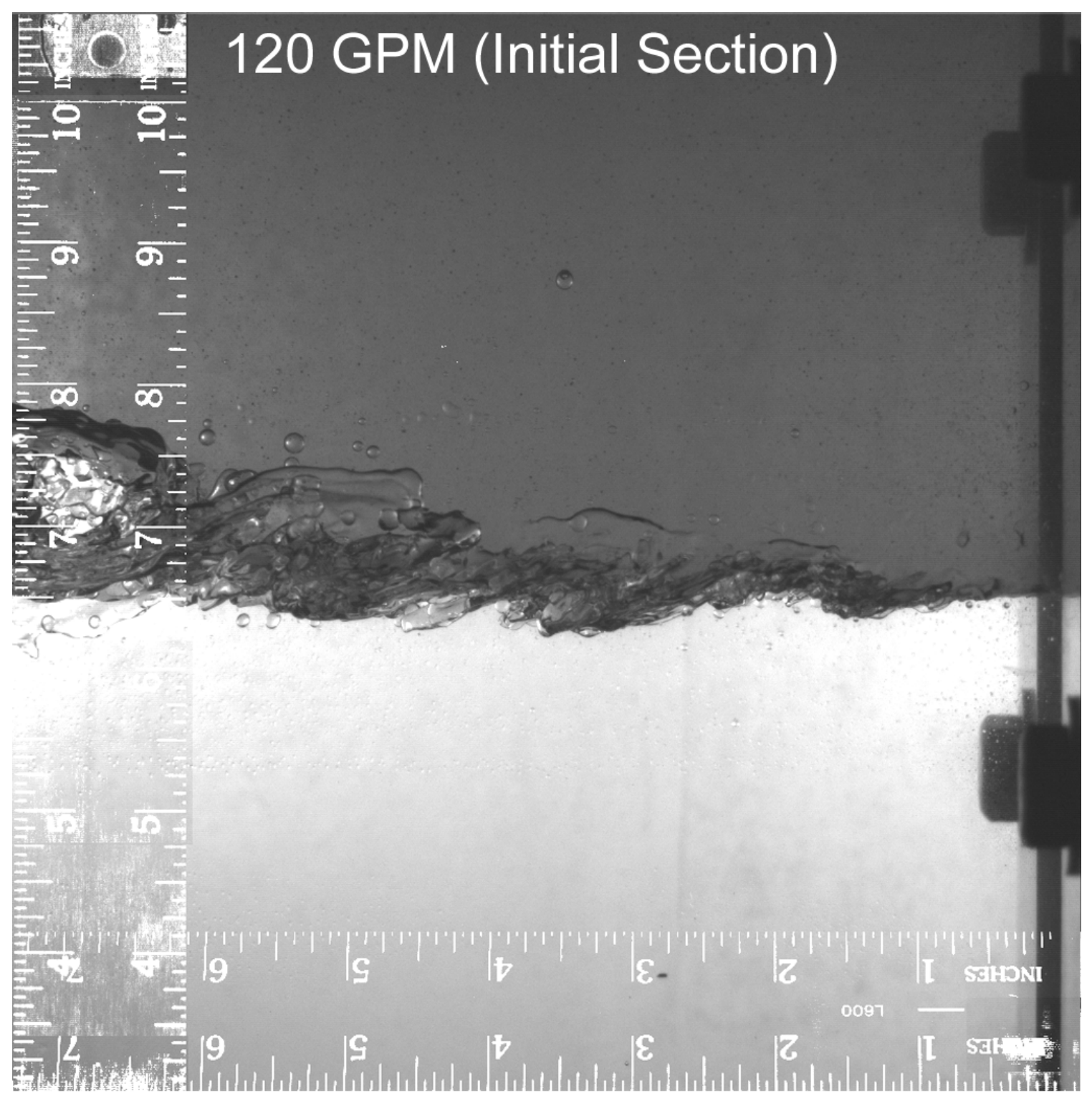

Figure 6.14 - Instantaneous mixing layer near water inlet; After Wu and Katz [38]. 


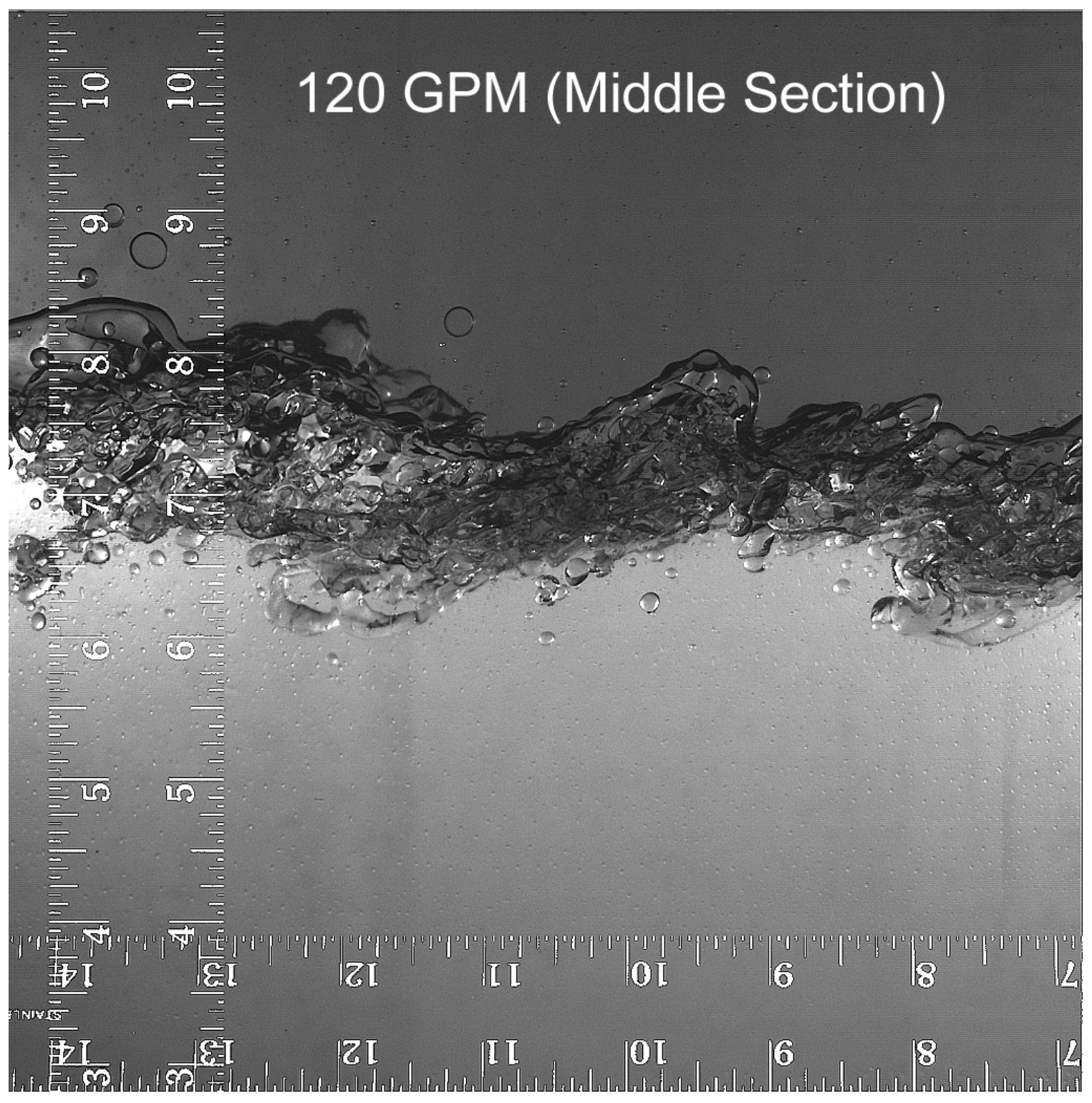

Figure 6.15 - Instantaneous mixing layer near central section; After Wu and Katz [38]. 


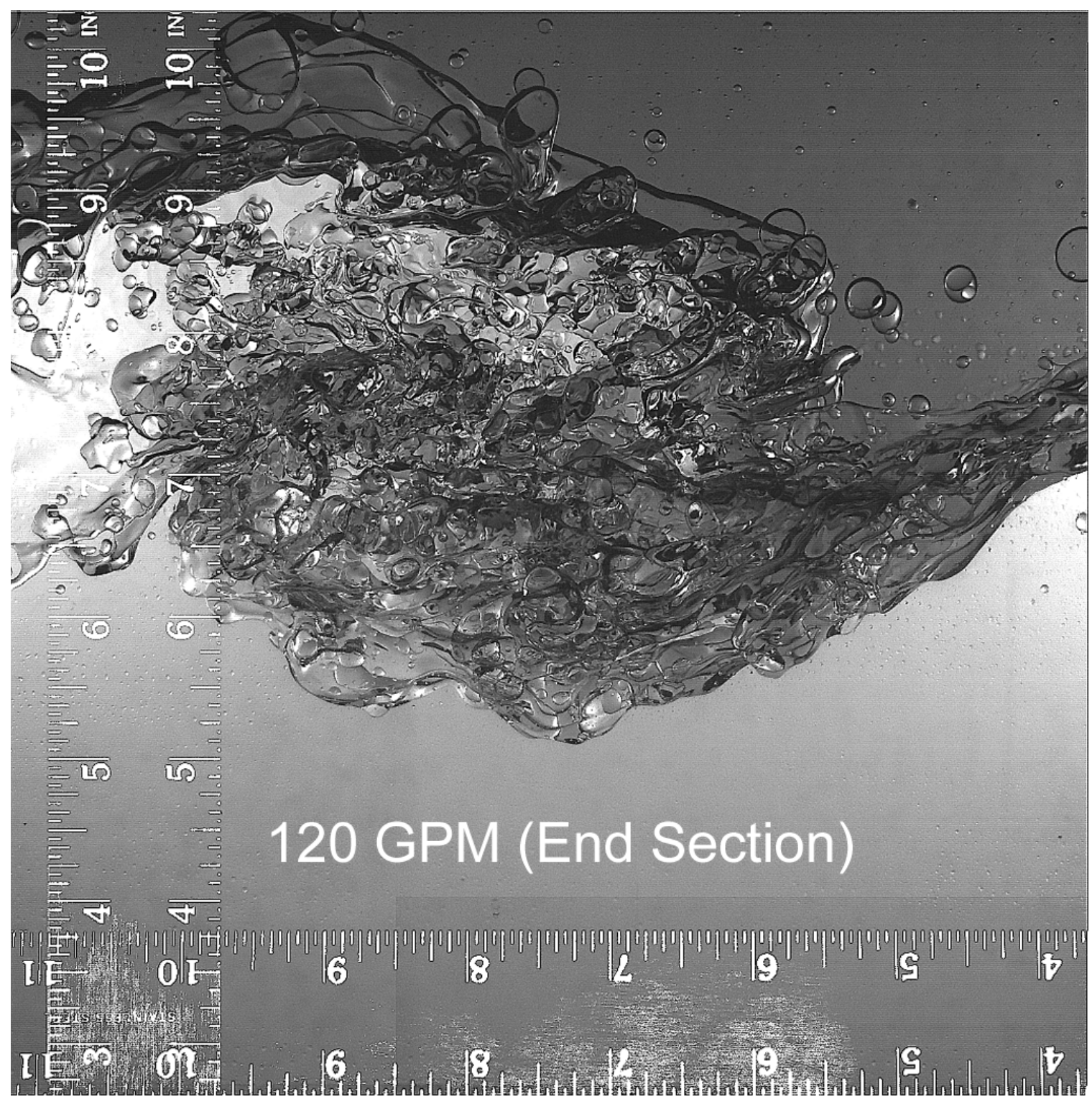

Figure 6.16 - Instantaneous mixing layer near fuel inlet diffuser; After Wu and Katz [38]. 
The experimental image given in Figure 6.14 shows the mixing layer near the water inlet, where the thickness of the mixed layer at $15.0 \mathrm{~cm}$ from the water inlet is approximately $3.0 \mathrm{~cm}$. This indicates that the value that was predicted in the simulations, which was also approximately $3.0 \mathrm{~cm}$, is reasonable in light of the experimental value.

The image taken at the middle section, as is shown in Figure 6.15, clearly shows the droplets that are formed at the interface. The various sizes of the droplets that are formed at the interface can be inferred from this image and give some credibility to the choices that were made for the average droplet size in the simulations. It should also be noted that the thickness of the mixed layer at this location, approximately $4.0 \mathrm{~cm}$, is also close to the value of the mixed layer thickness, $4.5 \mathrm{~cm}$, that was predicted in the simulations.

The image shown in Figure 6.16 shows the shear layer in the vicinity near the fuel inlet diffuser. In this profile, water is actually carried above the fuel layer and mixed in a type of Kelvin-Helmholtz structure. The re-circulation that is observed in this profile is similar to the mixing that is seen in the volume fraction profiles in Figure 6.13, near the fuel inlet diffuser. Hence, the time averaged profile of the experimental data near the fuel inlet diffuser, would probably look quite similar to the volume fraction profiles given in Figure 6.13. Both the structure that is shown in Figure 6.16 and the region of mixed fluid that is shown in Figure 6.13 have an approximate thickness of $9.0 \mathrm{~cm}$.

With regard to the shear at the interface, the zone of re-circulation occurring just after and above the fuel inlet diffuser tends to disrupt part of the shear layer. This is especially 
evident for the cases where $\mathrm{Ri}^{*}$ is low. The existence of this large re-circulation zone was experimentally confirmed [20] to exist, and will influence experimental data that is taken in the surrounding region. Fortunately, a region of developing shearing flow exists in the region beginning at the tip of the right splitter plate and ending approximately halfway between the fuel inlet diffuser and the right splitter plate. It is this density stratified, mixing layer that will be the main focus of the following discussion.

Specifically, consideration will be given to the influence of the gradient Richardson number and the assumed mean droplet size on several flow parameters of interest in the mixing layer. The development of the mixed fluid thickness, the interfacial thickness, and the maximum gradient Richardson number with increasing distance from the right splitter plate will be investigated. The interfacial thickness and mixed fluid thickness are defined in Eq. (5.1.5) and Eq. (5.1.9) respectively.

The profiles of the gradient Richardson number in the vertical direction will also be considered at various locations in the streamwise direction. From these profiles the influence of the gradient Richardson number and the influence of the average droplet size on several parameters related to the mixed layer will be inferred. No experimental data for values of the gradient Richardson number or the mixed layer thickness were available so the results from the simulations will be discussed in light of what is commonly observed in density stratified, turbulent mixing layers and flows involving immiscible fluids. 
The mixing layer is a common flow phenomenon, and some of the laws related to the mixed layer thickness for a turbulent mixing layer are useful in analyzing the current flow field. For a developing, two-dimensional, constant density, turbulent mixing layer the equation governing the spreading of the mixed layer is generally written as

$$
\frac{\mathrm{d} \delta_{\mathrm{M}}}{\mathrm{dx}}=\mathrm{C}
$$

where $\mathrm{C}$ is a constant. Figure 6.17 shows $\delta_{M}$ and shows a typical mixing layer. Here $\delta_{M}$ is the mixed layer thickness, which is defined by Eq. (5.1.9). For flows with no density stratification, $\delta_{M}$ is similar to the velocity layer thickness that is typically encountered in descriptions of turbulent mixing layers. The mixed layer thickness is shown here since it is the main parameter of interest in cases involving density stratification.

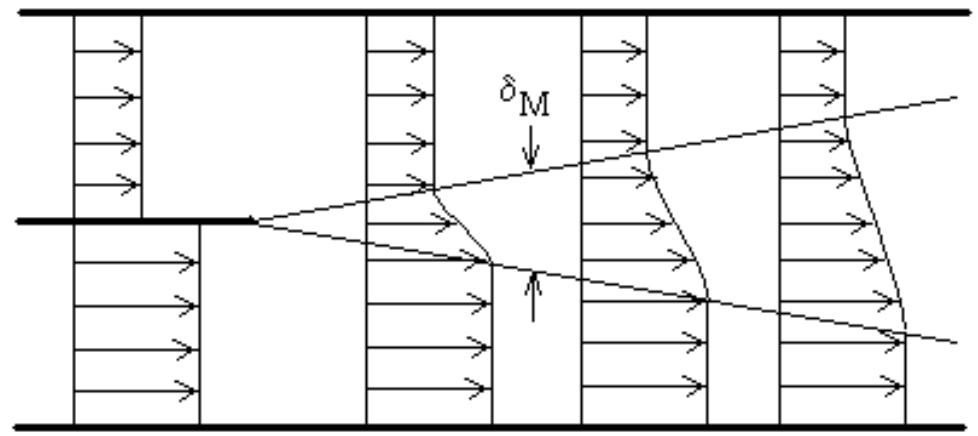

Figure 6.17 - Typical two-dimensional mixing layer. 
For a density stratified mixing layer the mixed layer thickness becomes a function of some appropriately chosen Richardson number [34]. This function is typically given by

$$
\frac{\mathrm{d} \delta_{M}}{\mathrm{dx}}=\operatorname{Ri}(\mathrm{x})^{\mathrm{n}}
$$

where $\mathrm{Ri}$ is defined here in a generic sense since it may be defined differently for different flow situations. Experimental studies indicate that values of the exponent $\mathrm{n}$ in Eq. (6.3.2) typically range from -0.25 to -2.0 . Some theoretical basis for Eq. (6.3.2) is also given in the literature [10]. It should be noted that for density stratified flows, the mixed fluid thickness and the velocity layer thickness that is shown in Figure 6.17 will not be equal. Figure 6.17 is used mainly to illustrate the flow field of interest and how $\delta_{\mathrm{M}}$ can be defined for a typical mixing layer. It should also be noted that there are two reasons for giving the functional form of $\delta_{M}$ based on the derivative with respect to the longitudinal distance. The first reason is because it removes the need to know how the Richardson number will change in the longitudinal direction, and the second reason is that it eliminates any arbitrary constants that might result from the integration.

For a density stratified mixing layer consisting of immiscible fluids, another parameter enters the law governing the mixed layer thickness to account for the presence of droplets and their slip velocity. Since the slip velocity is given functionally by

$$
\mathrm{u}_{\mathrm{s}}=f\left(\mathrm{r}_{\alpha}, \rho_{\mathrm{m}}, \mathrm{g}, \mu_{\mathrm{m}}, \mathrm{d}_{\mathrm{p}}\right)
$$

then all of the relevant variables are accounted for in the Richardson number, either implicitly or explicitly, except for $d_{p}$. Since the average droplet size will depend on the surface tension of the fluids, a Weber number dependence is implied. 
For droplets in turbulent dispersions the Weber number is typically defined by

$$
\mathrm{We}=\frac{\rho u^{2} \mathrm{~d}_{\mathrm{p}}}{\sigma}
$$

where $u=2 / 3 k^{1 / 2}[7]$. This leads to a function for $\delta_{M}$ that depends on the Richardson number and the droplet Weber number. It can be written in functional form as

$$
\frac{\mathrm{d} \delta_{\mathrm{M}}}{\mathrm{dx}}=f(\mathrm{Ri}, \mathrm{We})
$$

for a developing, density stratified, turbulent mixing layer involving immiscible fluids. No definite form of the entrainment law for immiscible fluids is given in the literature that was reviewed, and the present simulations are not intended to establish such a law or relationship. The simulations are intended to demonstrate that the current model does exhibit mixing relationships that are in agreement with Eq. (6.3.2), Eq. (6.3.3) and Eq. (6.3.5), depending on the flow scenario.

Since the gradient Richardson number is being used to characterize the predicted flow scenario, it will be considered in the region before the influence of the re-circulation. The main interest is how the gradient Richardson number changes along the shear layer. Placing the origin at the tip of the splitter plate on the right side and measuring positive $\mathrm{x}$ towards the left, the gradient Richardson number was calculated at various locations in the streamwise direction. For each of the cases, $\mathrm{Ri}_{\mathrm{G}}$ is shown at several $\mathrm{x} / \mathrm{L}$ locations in Figure 6.18 through Figure 6.21. $\mathrm{L}$ is the distance between the tip of the fuel inlet diffuser and the right splitter plate. For the case where $\mathrm{Ri}^{*}=0.51$ the profiles are only shown through $\mathrm{x} / \mathrm{L}=0.3$ since effects of the re-circulation zone were evident at larger $\mathrm{x} / \mathrm{L}$ values. 


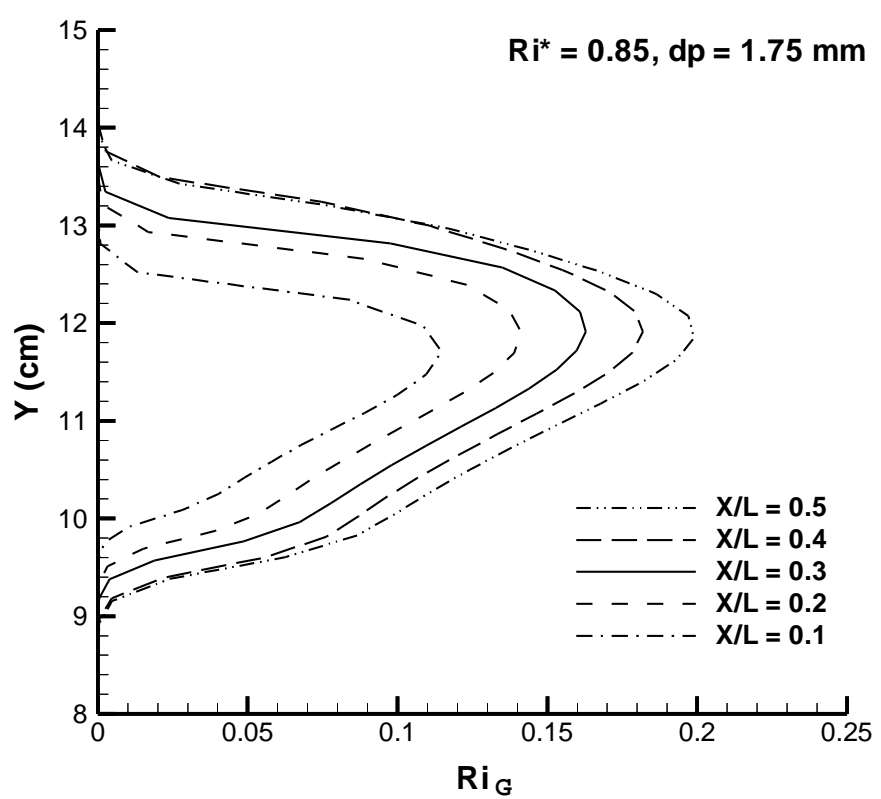

Figure 6.18 - Gradient Richardson number profiles for case 1.

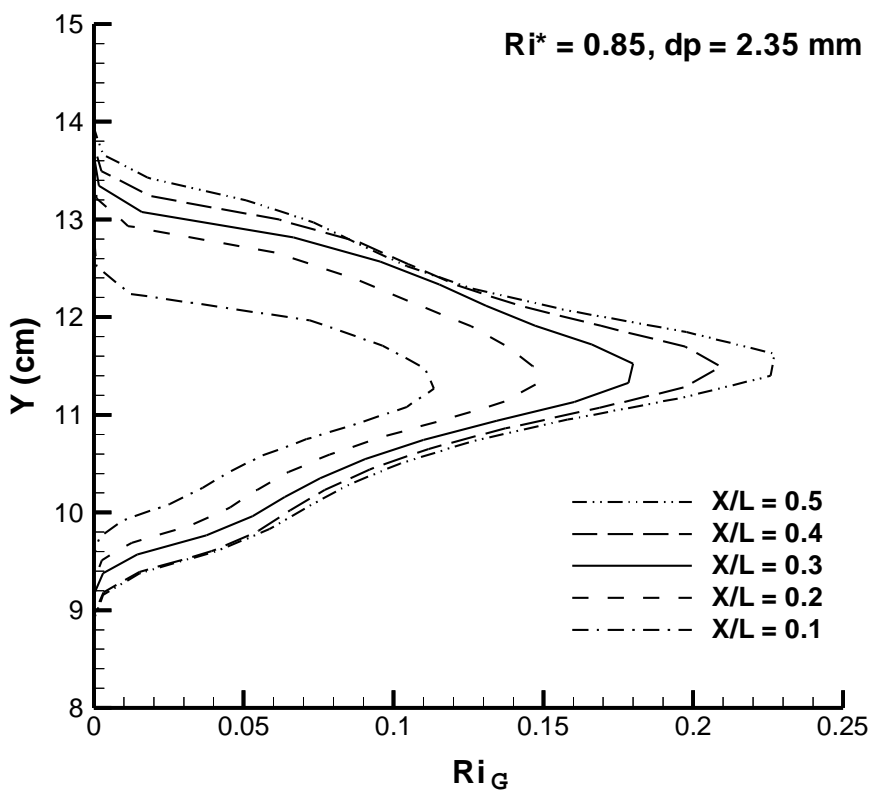

Figure 6.19 - Gradient Richardson number profiles for case 2. 


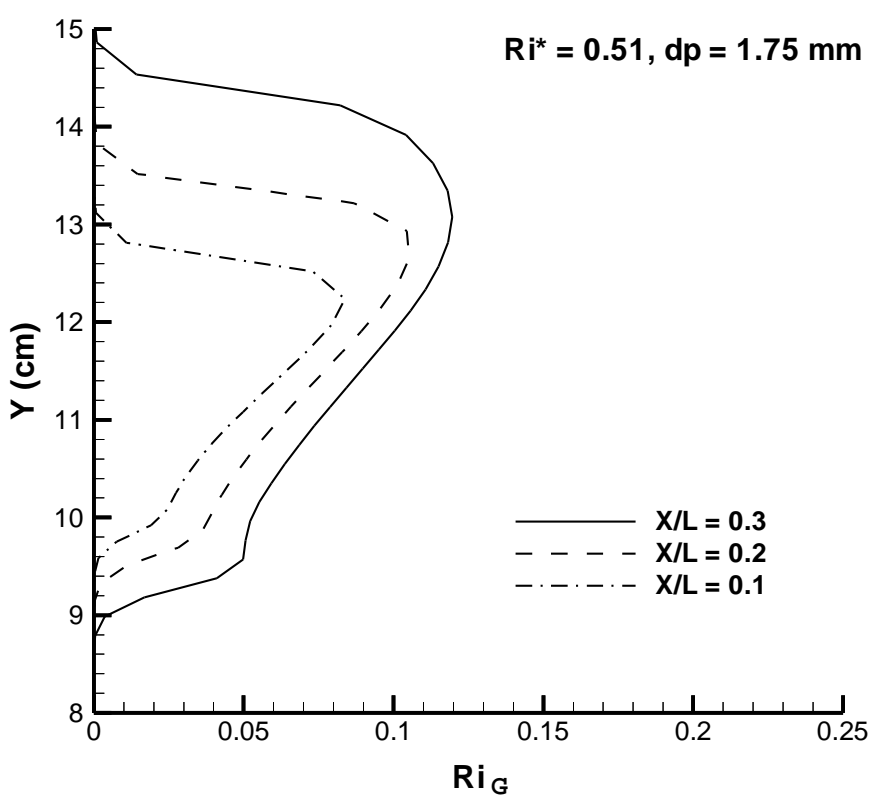

Figure 6.20 - Gradient Richardson number profiles for case 3.

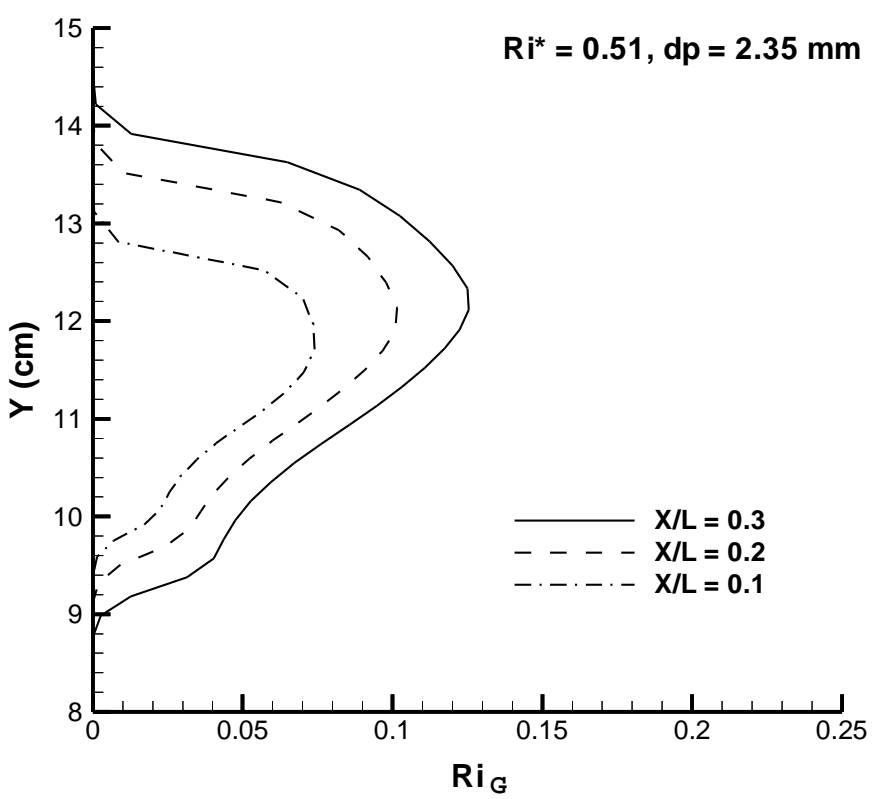

Figure 6.21 - Gradient Richardson number profiles for case 4. 
One import observation that can be made from every one of the four preceding figures is that $\mathrm{Ri}_{\mathrm{G}}$ increases with $\mathrm{x} / \mathrm{L}$. This tends to happen because of the velocity layer spreading and $(\partial U / \partial y)^{2}$ decreasing more quickly than $\partial \rho / \partial y$. Since $\operatorname{Ri}_{\mathrm{G}}$ will characterize the relevant scales of buoyancy and turbulence, its maximum value is a reasonable parameter to characterize the shear layer at various $\mathrm{x} / \mathrm{L}$ locations. Hence the maximum gradient Richardson number is plotted as a function of $\mathrm{x} / \mathrm{L}$ for all four cases in Figure 6.22. As in the previous plots, the trend for the maximum gradient Richardson number is shown over a smaller range of $\mathrm{x} / \mathrm{L}$ values for the cases where $\mathrm{Ri}^{*}=0.51$; this was done to avoid plotting values that were influenced by the re-circulation zone.

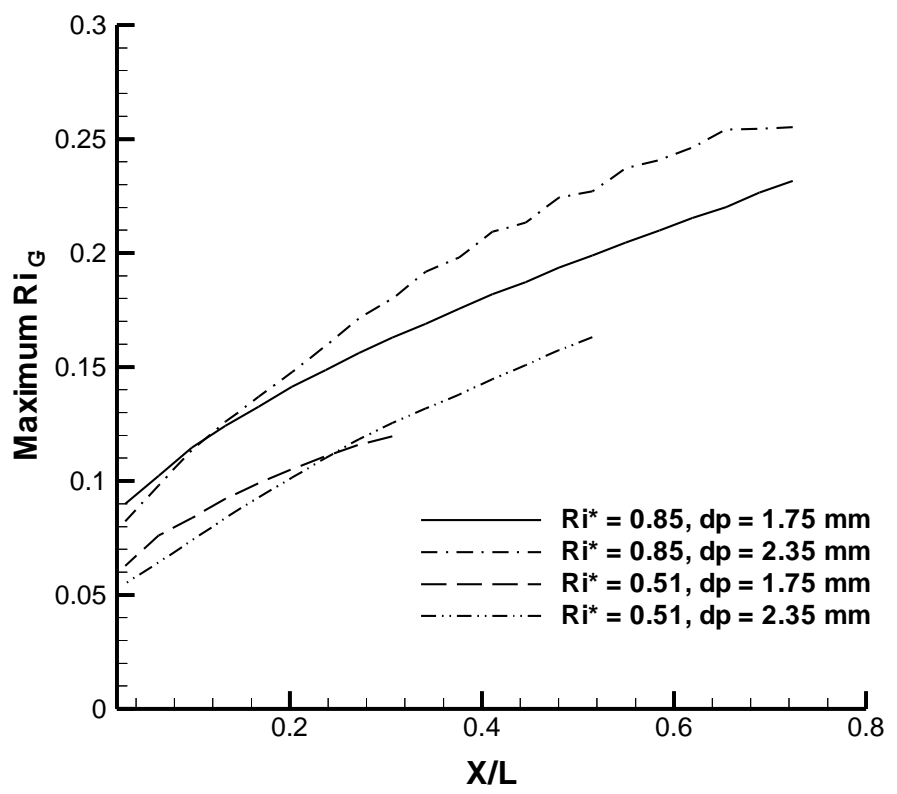

Figure 6.22 - Maximum gradient Richardson number versus x/L. 
Tennekes and Lumley [29] comment that for typical shear flows turbulence will persist at the interface for $\mathrm{Ri}_{\mathrm{G}}<0.2$. Miles and Howard comment that for unbounded, parallel shear flow if $\mathrm{Ri}_{\mathrm{G}}>0.25$ then no turbulent instabilities are observed near the interface as all turbulent motion is damped by the buoyancy [22]. However, for bounded flows the critical gradient Richardson number can be much lower than 0.25 , and the value of $\operatorname{Ri}_{\mathrm{G}}$ that will mark the transition from a fully turbulent interface to a non-turbulent or intermittent interface can be lower than 0.2 [10].

In consideration of these typical values, it should be reasonable to estimate from Figure 6.19 that the interface was most probably turbulent for the cases where $\mathrm{Ri}^{*}=0.51$. For the case where $\mathrm{Ri}^{*}=0.85$ the interface was probably characterized partially by KelvinHelmholtz waves which are typically the first stage observed after the fully turbulent interface.

In any case it is clear that since $\mathrm{Ri}_{\mathrm{G}}$ increases with $\mathrm{x} / \mathrm{L}$, that the mixed layer thickness and the interfacial thickness, defined by Eq. (5.1.5), should also change. The variation of predicted $\delta_{\mathrm{M}}$ with $\mathrm{x} / \mathrm{L}$ is shown in Figure 6.23 and the variation of $\delta$ with $\mathrm{x} / \mathrm{L}$ is shown in Figure 6.24. $\delta$ is shown here because it was a parameter of interest in the simulations involving the experiments performed by Sullivan and List, and because its value may indicate how the parameters related to mixing will behave near the interface. 


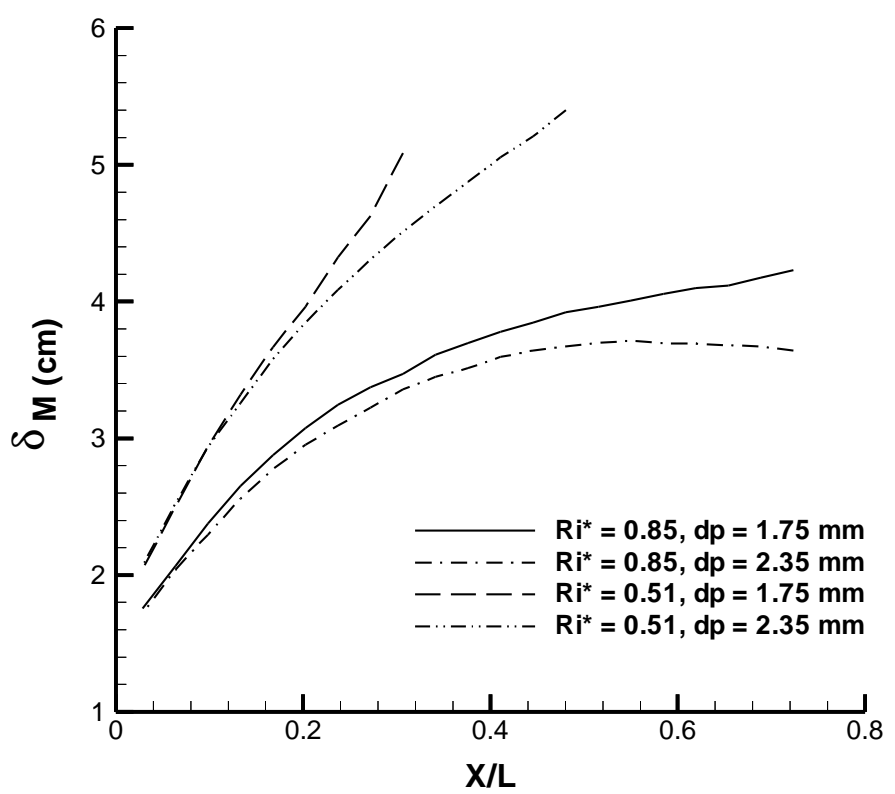

Figure 6.23 - Mixed fluid thickness versus x/L.

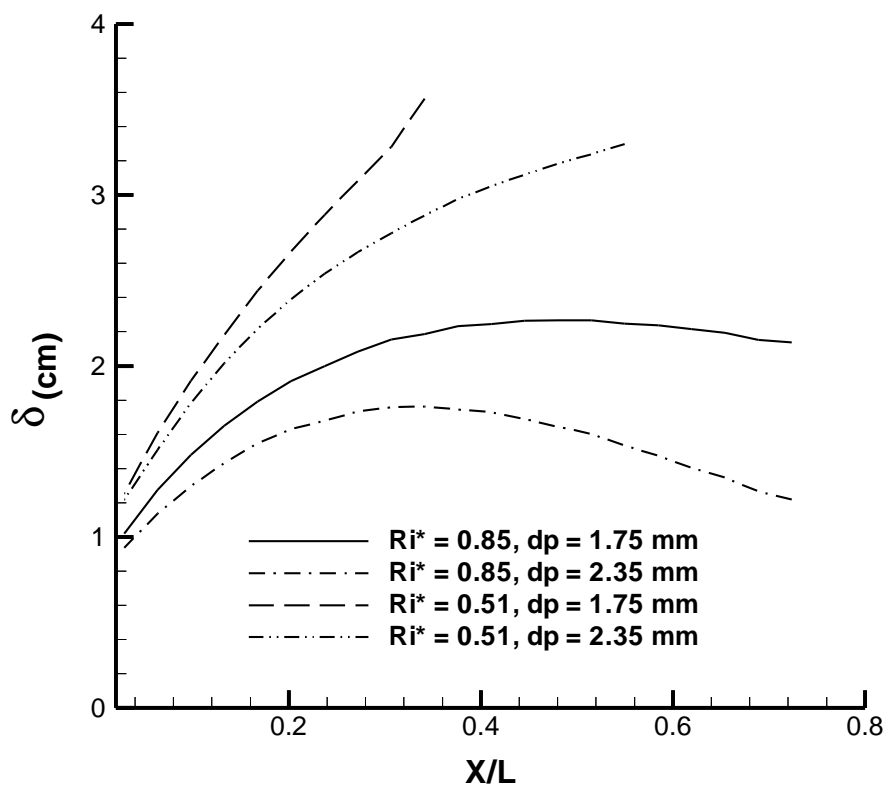

Figure 6.24 - Interfacial thickness versus x/L. 
How $\delta_{\mathrm{M}}$ changes with $\mathrm{x} / \mathrm{L}$ is interesting because all three laws for the mixed layer thickness appear to be demonstrated. For the case given by $\mathrm{Ri}^{*}=0.51$ and $\mathrm{d}_{\mathrm{p}}=1.75 \mathrm{~mm}$, the maximum gradient Richardson number is approximately 0.1 over the horizontal distance where $\delta_{\mathrm{M}}$ was calculated for this case. Since this is a low gradient Richardson number and the effects of the turbulence dominates the layer, $\delta_{\mathrm{M}}$ has a slope that is approximately constant. This is shown by the heavy dashed line in Figure 6.23. This is characteristic of a mixing layer with no density stratifications. The effect of the slip velocity is to decrease $\delta_{\mathrm{M}}$. For $\mathrm{Ri}^{*}=0.51$ and $\mathrm{d}_{\mathrm{p}}=2.35 \mathrm{~mm}$, the effect of the average droplet size or the slip velocity is to bend the almost linear curve toward the right as $\mathrm{x} / \mathrm{L}$ increases.

The relative effects of increasing $\mathrm{Ri}_{\mathrm{G}}$ are shown by the solid curve in Figure 6.23, which corresponds to $\mathrm{Ri}^{*}=0.85$ and $\mathrm{d}_{\mathrm{p}}=1.75 \mathrm{~mm}$. The shape of this curve demonstrates that $\delta_{\mathrm{M}}$ asymptotically approaches a constant as $\mathrm{Ri}_{\mathrm{G}}$ increases. This is consistent with the experimental observation that $\mathrm{d} \delta_{\mathrm{M}} / \mathrm{dx} \sim \mathrm{Ri}^{-\mathrm{n}}$ for some Richardson number giving a measure similar to that given by $\mathrm{Ri}_{\mathrm{G}}$.

The influence of the slip velocity is to actually separate the mixed fluid layers. The effect of increasing the average droplet size, which could be accomplished by changing the surface tension of the fluids, on $\delta_{\mathrm{M}}$ can be seen in Figure 6.23. However, the effects of $d_{p}$ are most clearly seen in Figure 6.24. In Figure 6.24 the interfacial thickness, $\delta$, is shown to first increase and then decrease. The cause for this is two fold. Initially, $\mathrm{Ri}_{\mathrm{G}}$ is relatively small and the turbulence begins mixing the layers. As $\operatorname{Ri}_{\mathrm{G}}$ increases the 
turbulence is damped and $\delta$ does not increase significantly with $\mathrm{x} / \mathrm{L}$. Finally, as the turbulence decreases still more, the slip velocity begins to separate the layers, causing $\delta$ to decrease. This trend is essentially the same for $\delta$ and $\delta_{M}$, though it is more pronounced for $\delta$. This seems to partially originate from the fact that very near the interface, where $\delta$ is measured, the turbulence is more heavily damped by the buoyancy effects. The manner in which the thickness of the mixed layer and the interfacial thickness first increases and then decreases has been experimentally confirmed by the experiments at Johns Hopkins [38]. For several of the cases that have been experimentally investigated this behavior has been observed. Hence, in addition to substantiating the trend in the simulations, this confirms the importance of the slip velocity in the model equations.

In general, the simulations performed with SFST2 give reasonable predictions for the overall flow field and the parameters related to the mixed layer thickness. The predicted flow field was confirmed to qualitatively represent the flow field that was observed at the shear flow facility at Johns Hopkins [38]. This comparison was done using several instantaneous profiles of the mixed interface at various streamwise locations along the shear layer. Average sizes for the droplets and the influence of the zone of re-circulation near the fuel inlet diffuser were also considered. The manner in which $\delta$ and $\delta_{\mathrm{M}}$ first increase and then decrease also agrees with what has been observed in the experiments at Johns Hopkins [38]. 


\subsection{SIMULATION OF FLOW INSIDE A FULL SCALE CFBT}

The transient filling of a multi-compartment, compensated fuel ballast tank (CFBT) was investigated using CFX 4.1 and the SFST model for immiscible fluids, SFST2. As mentioned in the introduction, the overall project objective was to develop a computational tool that could be used for predicting fuel and water mixing in a CFBT. Though certain aspects of the model remain to be refined, this chapter demonstrates the capability of SFST2 to simulate the actual flow through a CFBT. In these simulations the primary areas of interest were the extent to which the fuel and water mix and the locations inside the CFBT where the most mixing occurs. Another objective involved predicting the amount of water that is trapped inside the compartments between the compartment floor and the fuel layer.

\subsection{Actual Flow Conditions and Computational Model}

The CFX model was based on the full scale DDG 51 Tank 5-300-2-F Compensated Fuel/Ballast Tank as is described by Chang [5]. A schematic of DDG 51 Tank 5-300-2-F is shown in Figure 1.1. In the computational model, the limberholes and the transverse and longitudinal slope of the tank floor were neglected. These simplifications to the geometry were made because the primary goal was to investigate the applicability of SFST2 to a transient, three-dimensional, geometry at full scale, not to match every geometric detail in the actual tank. The geometry for the model that was simulated is shown in Figure 7.1, where the inlet, outlet, transverse manholes, and longitudinal manholes are labeled. In Figure 7.1 the front and top walls are shown to be transparent to show the internal structures that were included in the model. The dimensions of the tank 
were $12.0 \times 2.7 \times 1.4$ meters. The inlet and outlet were modeled as rectangular instead of round to aid in obtaining better numerical convergence at the inlet and outlet regions where a significant portion of the flow takes place.

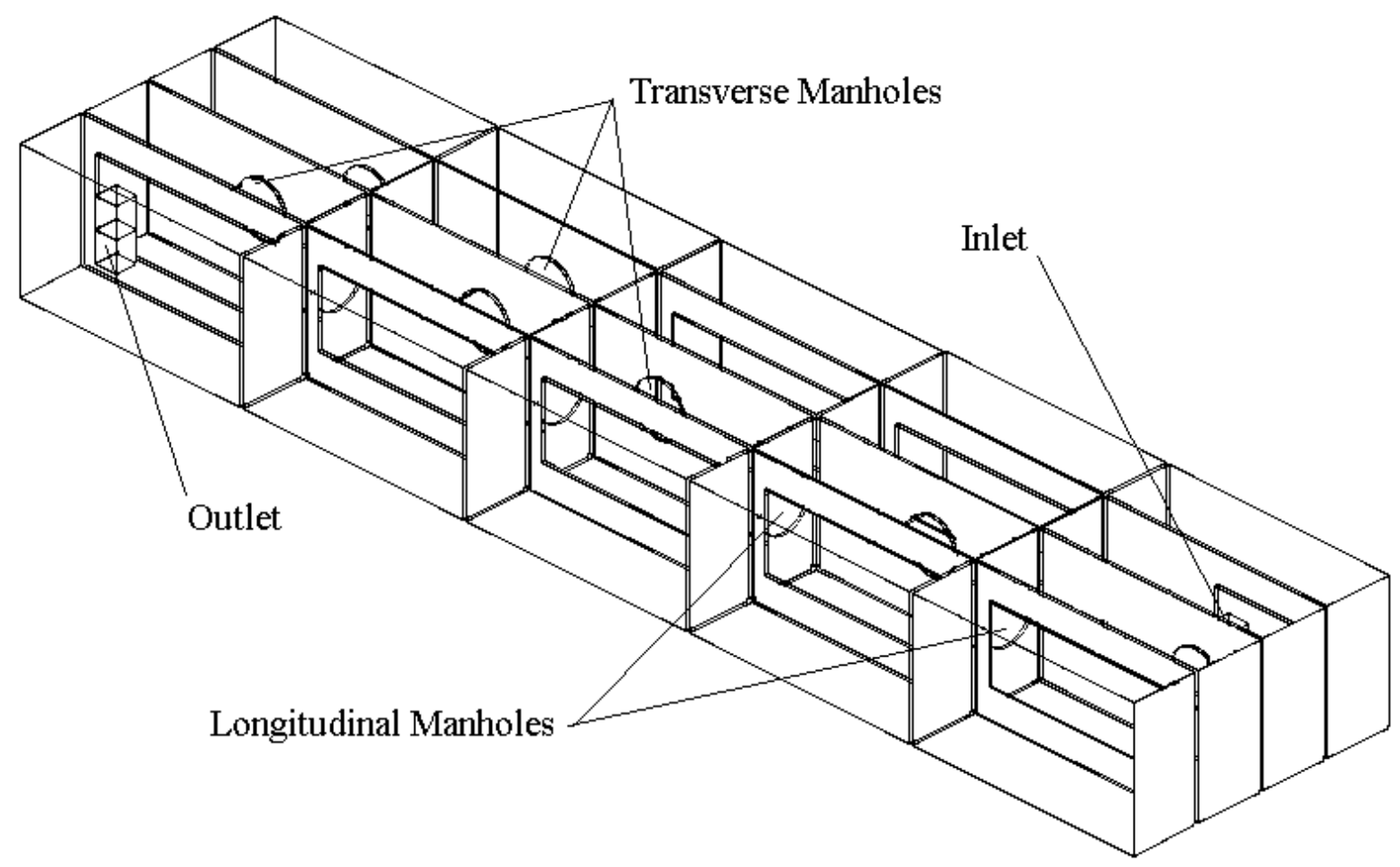

Figure 7.1 - Geometry of CFBT model that was simulated in CFX.

As already described in Chapter 1.0, during refueling diesel fuel is pumped into the tank through a vertical inlet pipe as is shown in Figure 7.1. The water is forced out of the bottom of the tank through a vertical outlet pipe in the last compartment. 


\subsection{Computational Details for DDG 51 Tank 5-300-2-F Simulations}

The SFST model for immiscible fluids, SFST2, was used to model the mixing of the fuel and water. In the formulation of the SFST model that was used, the mixture definitions, momentum equations, and mass conservation equations as presented in Section 3.3 were adopted as the governing equations. The constitutive equation for the relative motion between the phases in the vertical direction was assumed to be given by Eq. (6.2.6), and it was assumed that the relative motion between the phases was zero in the horizontal plane. An average droplet size of $3.5 \mathrm{~mm}$ was used in the slip velocity equation; this droplet size was based on typical droplet sizes that were observed in some of the experiments at Johns Hopkins. The drift flux terms were also neglected as was done in Section 6.2, giving the momentum equations that were used in the present simulations by Eq. (6.2.8). The $k$ - $\varepsilon$ model with the added buoyancy terms, as described in Section 4.3, was also used.

In addition to using the SFST model, the default homogeneous model in CFX was used to simulate the flow. The CFX homogeneous model is somewhat similar to SFST1, which was presented in Section 3.2. After performing several computational experiments, it was determined that the default CFX homogenous model was actually about $30 \%$ to $40 \%$ slower computationally than SFST1 or SFST2. The default homogeneous model was also difficult to converge on the multi-compartment tanks.

The grid in each compartment consisted of 32 longitudinal cells, 9 transverse cells, and 18 vertical cells. Including the cells in the inter-compartment passages such as manholes, 
the total grid was approximately 110,000 cells. In each compartment, the cells were concentrated towards the inter-compartment manholes and internal openings using a symmetric geometric progression factor of 1.06 in the longitudinal direction and 1.1 in the transverse direction. Also, above the inlet pipe and below the outlet pipe the grid was made twice as fine in the vertical direction to better resolve the inlet and outlet jets of fluid. The grid near the inlet is shown in Figure 7.2, where the internal block boundaries are also visible. Figure 7.3 shows a typical section of the transverse grid, where the fine grid near the inlet can be seen in the third block from the left at the top of the geometry.

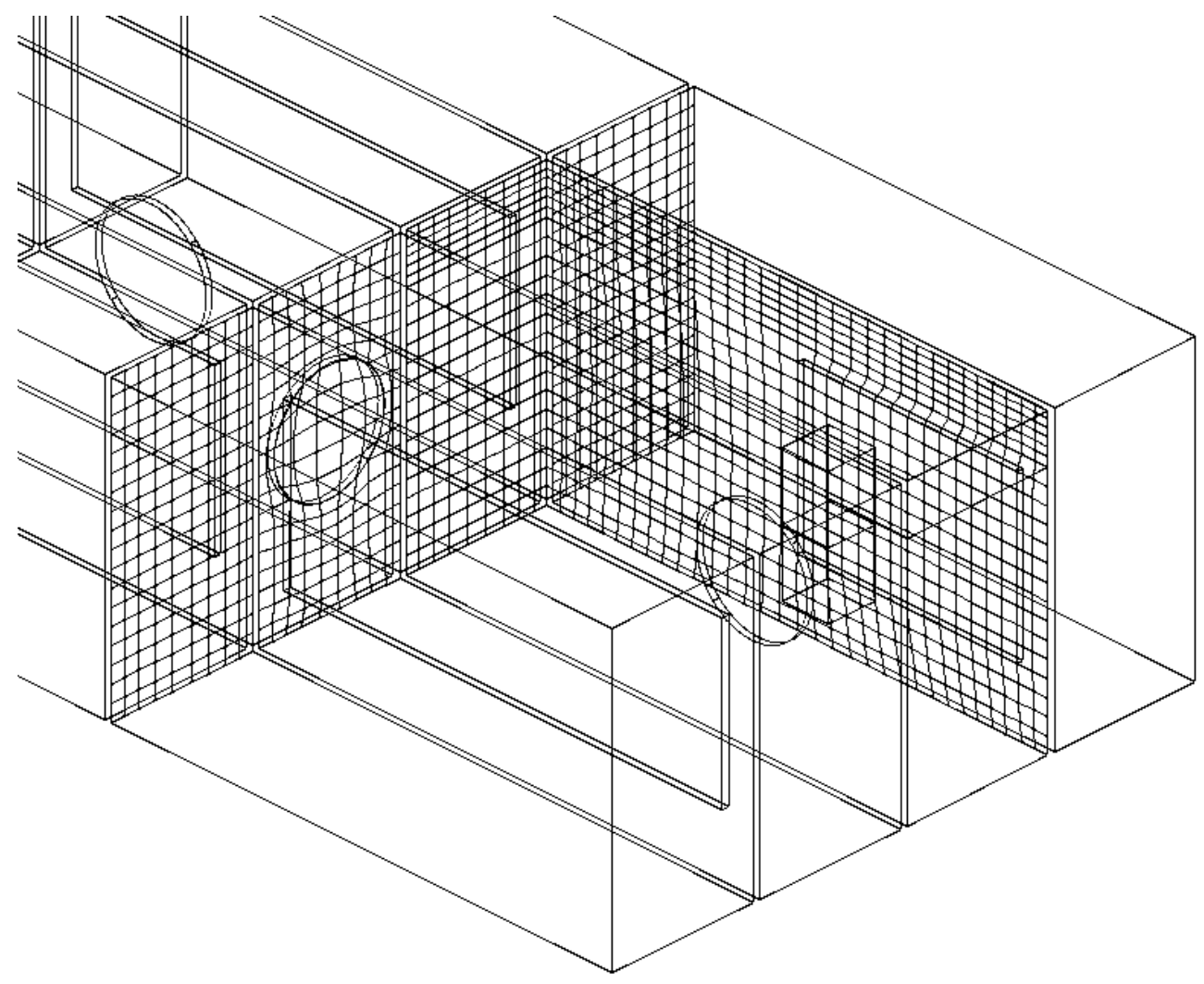

Figure 7.2 - Schematic of the computational grid near the inlet. 


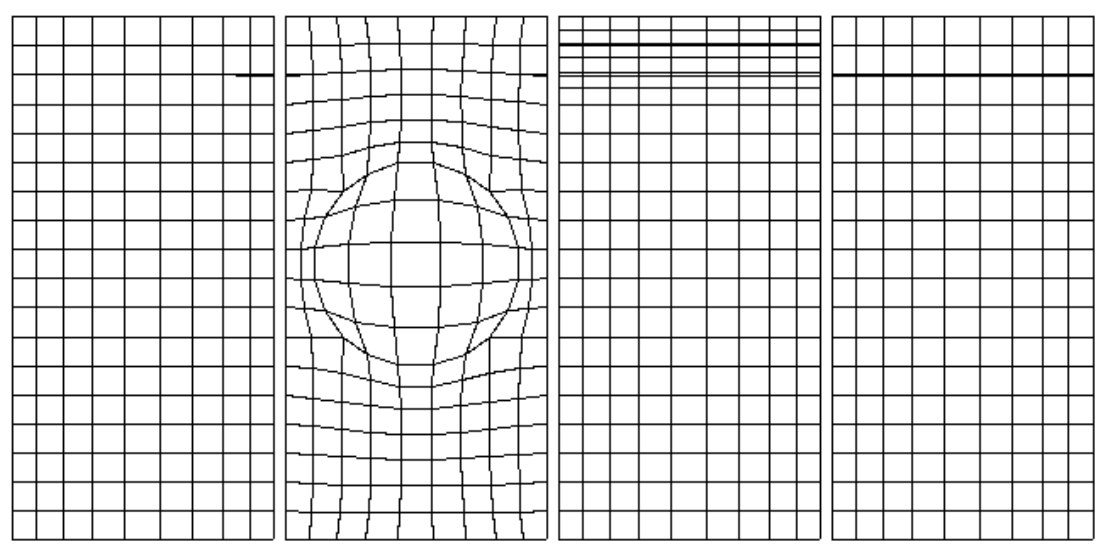

Figure 7.3 - Transverse plane showing grid near inlet compartment.

Initial conditions for the model consisted of a quiescent tank filled entirely with water. Fuel entered the tank through the inlet pipe and forced the water out through the outlet pipe. Boundary conditions for the inlet were modeled by setting a fixed velocity that corresponded to 100.0 liters per second, which is a typical flow rate in a re-fueling process. This inlet volume flow rate corresponded to a velocity of approximately $2.0 \mathrm{~m} / \mathrm{s}$ at the inlet. Based on the tank volume and the inlet volumetric flow rate, the flow through time was equal to 425.0 seconds. The density of the unmixed fuel was set equal to 850.0 $\mathrm{kg} / \mathrm{m}^{3}$ and the density of the unmixed water was set equal $1,000.0 \mathrm{~kg} / \mathrm{m}^{3}$. The volume fraction at the inlet was set equal to 0.99999 , corresponding to pure fuel entering the tank. Values of $k$ and $\varepsilon$ were estimated at the inlets using Eq. (5.2.1) and Eq. (5.2.2) and pressure at the inlets was extrapolated from downstream. Hence Dirichlet boundary conditions were specified on all quantities except pressure at the inlet. Boundary conditions at the outlets were modeled by setting Neumann conditions on all the variables 
except pressure, which was fixed at 0.0 Pascals at the outlet. The FORTRAN which was used to implement the additional source terms, user functions, and user boundary conditions for SFST2 and documentation for using the subroutines has been included in Appendix B.

In an effort to make the numerical convergence of the model easier and decrease the computational time, first order upwinding was used to discretise all of the variables except pressure. The pressure was discretised using central differencing. The first order Euler scheme was used to advance the solution in time. The time steps used for the simulations were chosen such that the Courant limit was satisfied for the early part of the simulation, and then the time step was increased as the simulation progressed in time. The initial time step was 0.1 seconds, and the time step was increased to 0.25 , then 0.5 , and finally 1.0 seconds.

An important reason to use small time steps initially is because the sudden inflow of fuel into the quiescent water is similar to a sharp step input to the velocity field. Since the fuel and water are incompressible, the solution must find a velocity field that satisfies the conservation laws and discharges an amount of volume of fluid that is equivalent to the amount pushed in on the first time step. This is significantly easier if small time steps are used at the beginning of the simulation. 


\subsection{Results and Discussion}

Figure 7.4 through Figure 7.7 show fuel volume fraction contours and velocity vectors at a transverse plane across the inlet, at various times in the simulation. Figure 7.4 shows a contour of the fuel volume fraction near the inlet after 5.0 seconds, with the tank outline shown and the inlet pipe indicated by a white rectangle. Figures 7.5, 7.6, and 7.7, show contours of the fuel volume fraction and velocity vectors at 5.0, 15.0, and 25.0 seconds, at the same location as the contour shown if Figure 7.4. The maximum velocity at the inlet in each of these figures is $2.0 \mathrm{~m} / \mathrm{s}$. These figures clearly demonstrate the transient nature of the re-fueling process, and the mixing and separation of the two fluids during the refueling.

Results (see Figures 7.4 - 7.7) from the simulation of the CFBT using SFST2, where the terms containing the slip velocity are included, are very encouraging as they predict a distinct interface between the oil and water. This distinct interface is expected in the actual re-fueling scenario. This differs substantially from the predictions given by the default, CFX homogenous multiphase model, where the two fluids constantly blend into one another, forming an emulsion phase with no distinct interface. Similar results have been obtained with SFST1, where no slip velocity is accounted for. In both SFST1 and the CFX homogeneous model the fluids mix and never separate, in essence forming a single fluid with a stably varying density. This indicates the importance influence of the vertical slip velocity in these flow scenarios involving oil and water. 


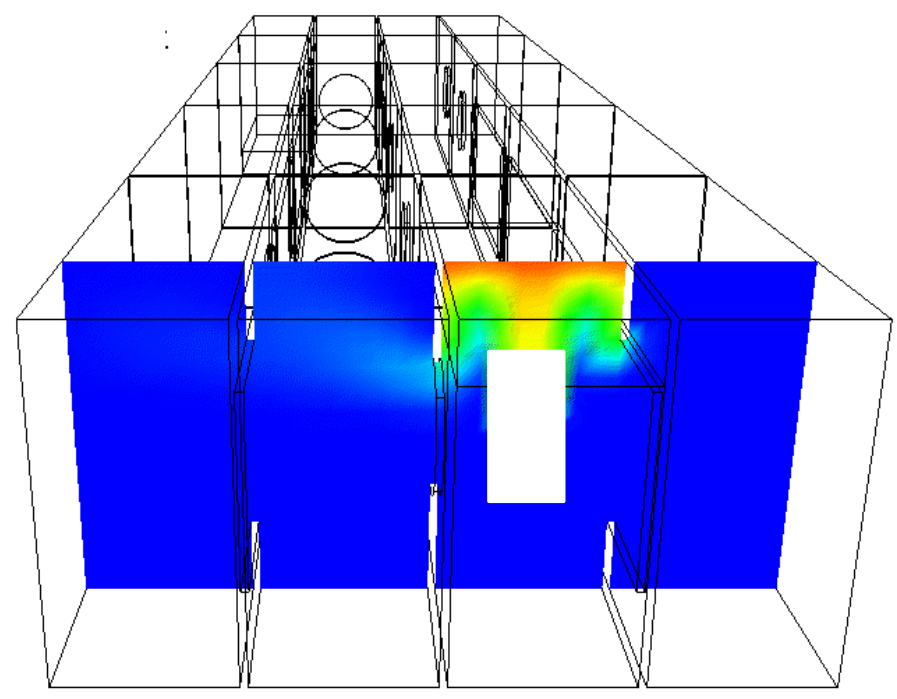

Volume Fraction

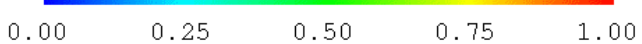

Figure 7.4 - Fuel volume fraction contours near inlet after 5.0 seconds.

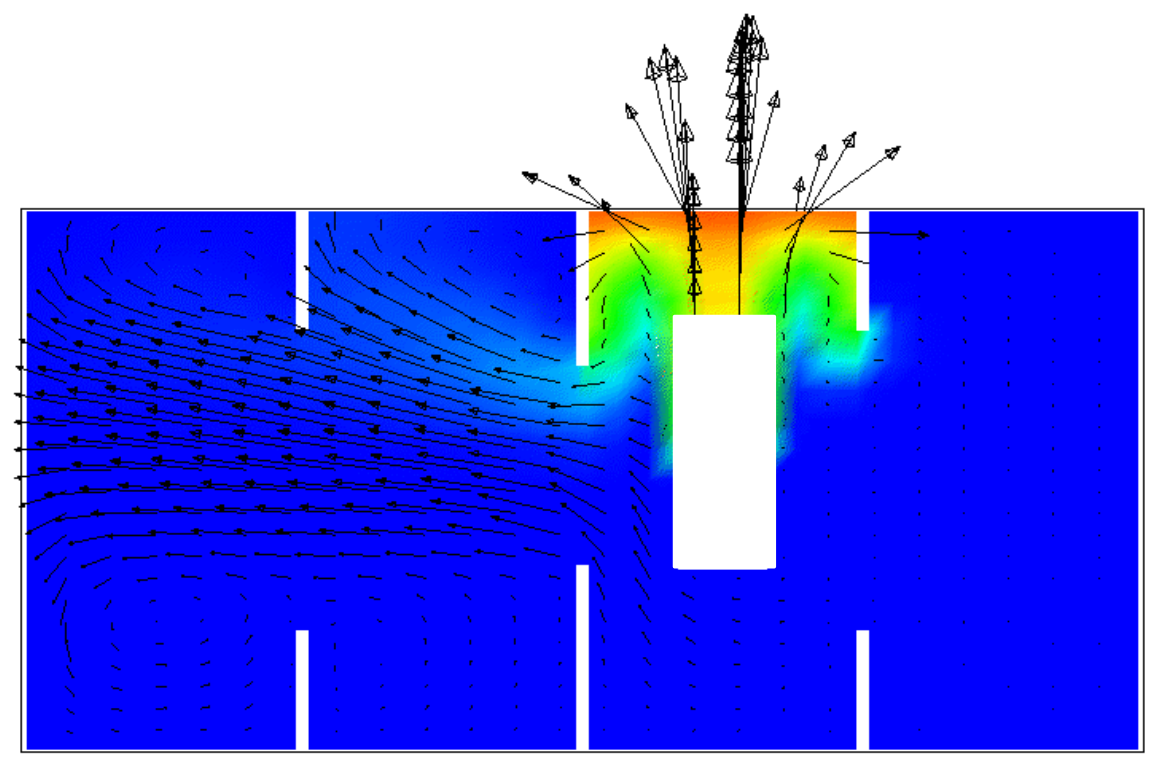

Volume Fraction
0.00
0.25
0.50
0.75
1.00

Figure 7.5 - Fuel volume fraction contours and velocity vectors after 5.0 seconds. 


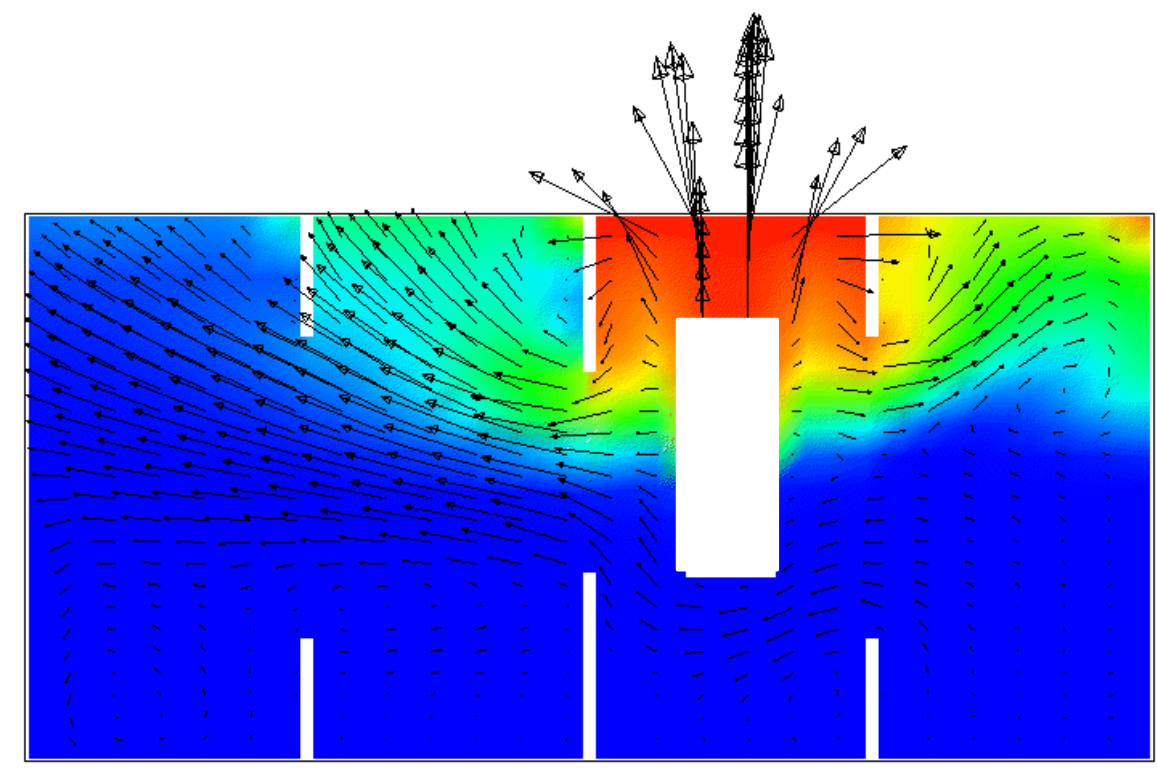

Volume Fraction
0.00
0.25
0.50
0.75
1.00

Figure 7.6 - Fuel volume fraction contours and velocity vectors after 15.0 seconds.

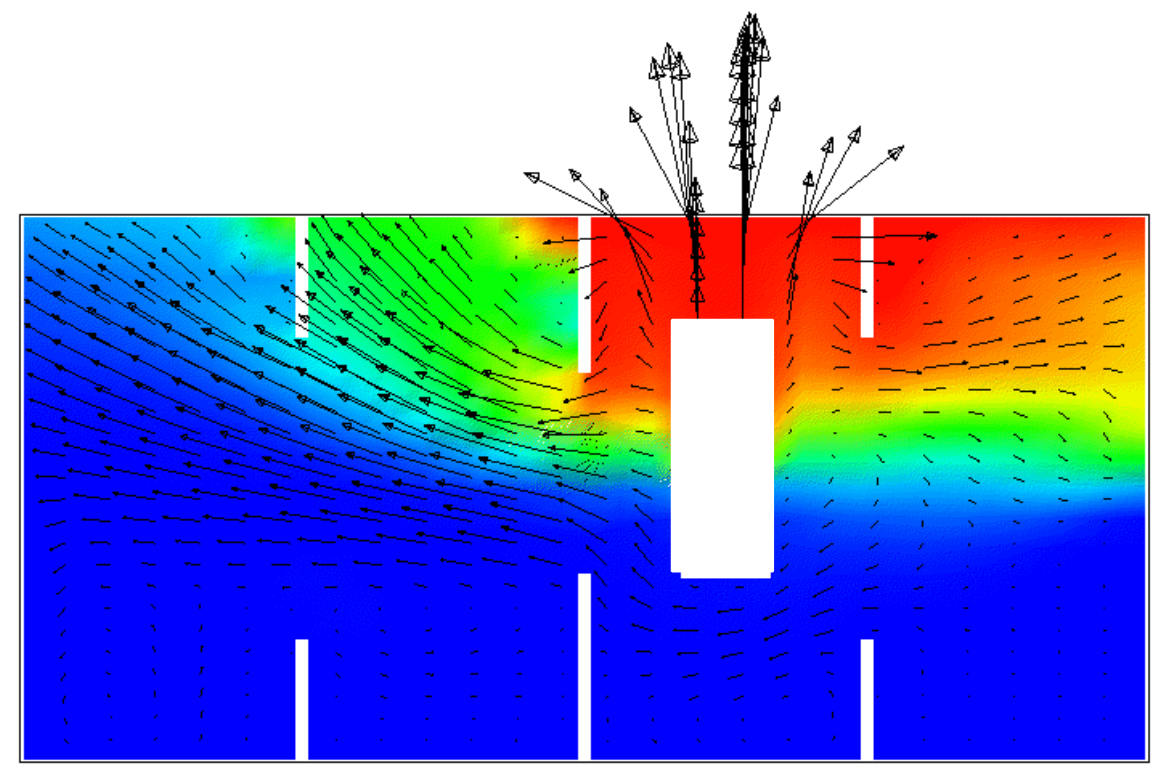

Volume Fraction
0.00
0.25
0.50
0.75
1.00

Figure 7.7 - Fuel volume fraction contours and velocity vectors after 25.0 seconds. 
One important mixing event can be seen in Figure 7.6 in the second compartment from the left. When the fuel flows from a partially filled compartment into an empty compartment through a manhole or rectangular opening, a buoyant mixing event occurs. As the fuel layer reaches a certain depth it begins to spill into the second compartment under the top of the manhole or rectangular opening. During this event a buoyant jet of fuel issues into the water in the second compartment, dramatically increasing the mixing potential between the fluids. These occurrences have been labeled buoyant mixing events by Chang and his co-workers, who also identified such events in their simulations at NSWC [5]. Though the objective of the present simulation was not to suggest design modifications to the internal structure of the tanks, it is clear that these buoyant mixing events should be minimized in any attempt to re-design the tank.

The evolution of the fuel and water through the tank can be demonstrated by monitoring the flow rates of fuel and water through the longitudinal manholes. During the course of the simulations, several of these flow rates were computed at various manholes, which are labeled in Figure 7.8. Figure 7.8 also shows the geometry as if it were transparent, with the internal manholes and openings shaded. Flow rates from one transverse manhole and three longitudinal manholes were computed. These flow rates are shown in Figure 7.9 through Figure 7.12. The flow rates through manholes 1 and 2 almost reach a steady state where only fuel is going through the manholes as is shown in Figure 7.9 and Figure 7.10. An interesting time scale that is associated with the flow through a manhole is the time interval between when the fuel first begins to flow through a manhole and when the fuel flow rate overtakes the water flow rate. This time scale increases if a manhole is 
farther away from the inlet. The reason that this time scale increases depends on at least two factors: the amount of water that the fuel must displace in upstream compartments and the resistance the fuel will encounter in displacing the water in upstream compartments. Farther away from the inlet there is more water that remains to be displaced after the fuel initially flows through a manhole. Hence, the actual time it will take for the fuel to first reach a manhole and actually dominate the flow through the manhole will increase as the distance from the inlet to the manhole increases.

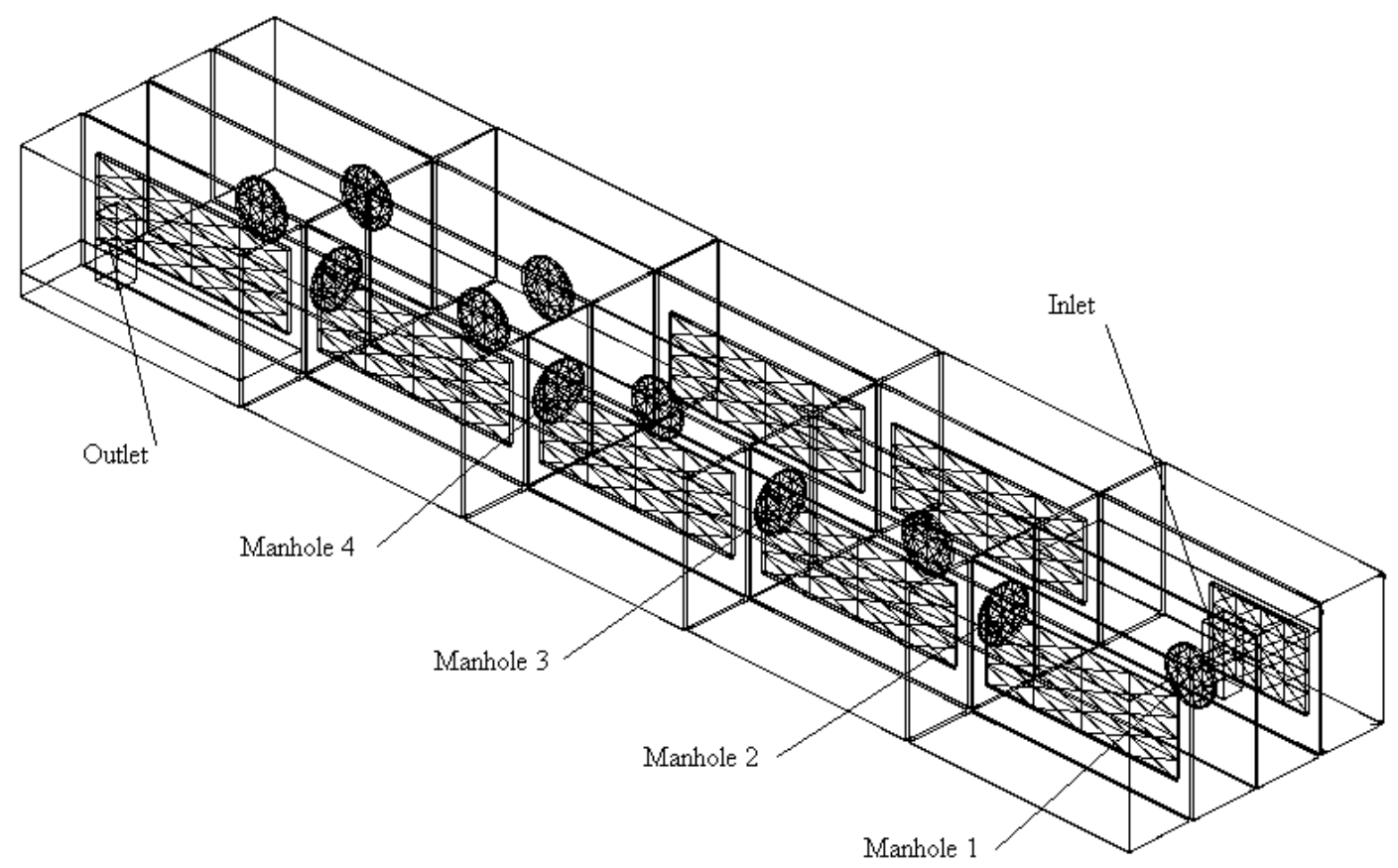

Figure 7.8 - Wireframe schematic of CFBT highlighting manholes and internal openings 


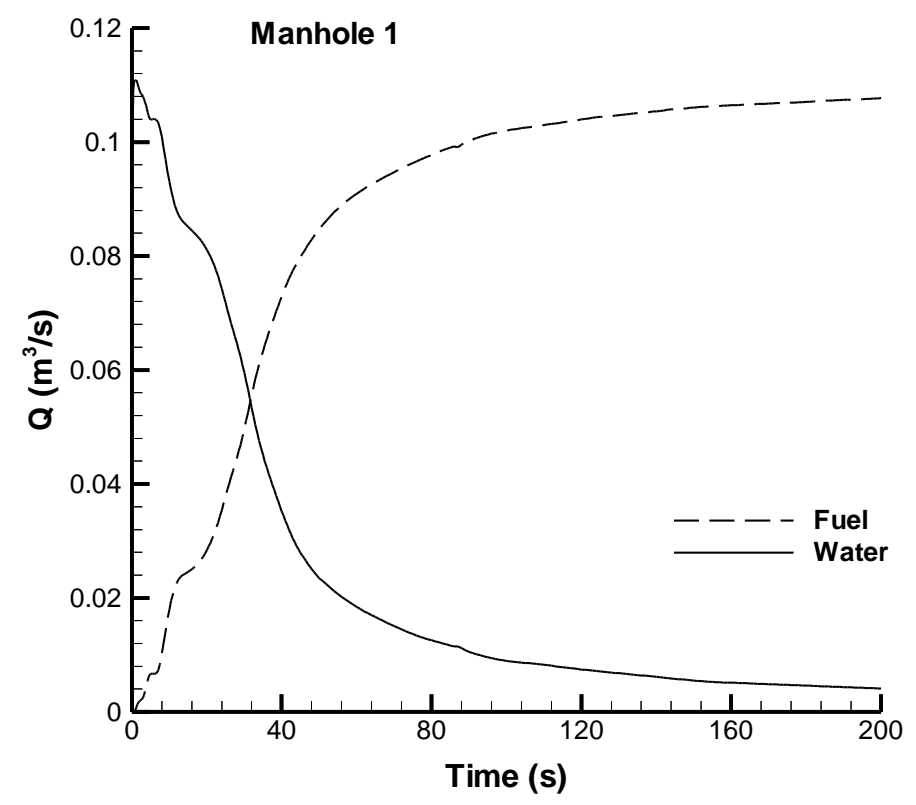

Figure 7.9 - Flow rates of fuel and water through manhole 1.

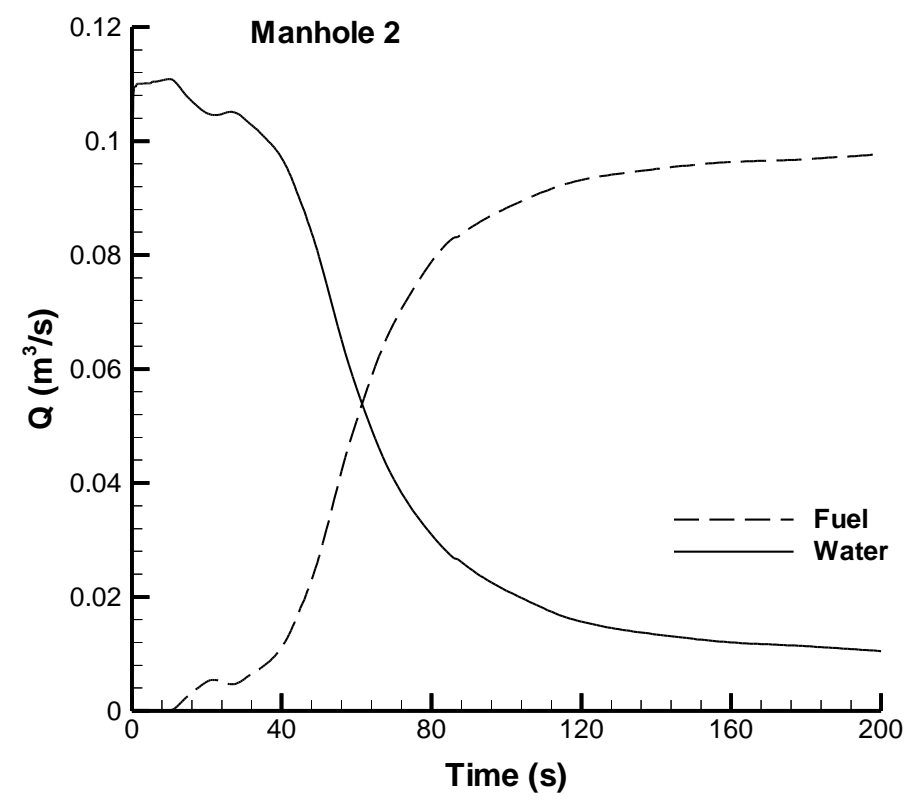

Figure 7.10 - Flow rates of fuel and water through manhole 2. 


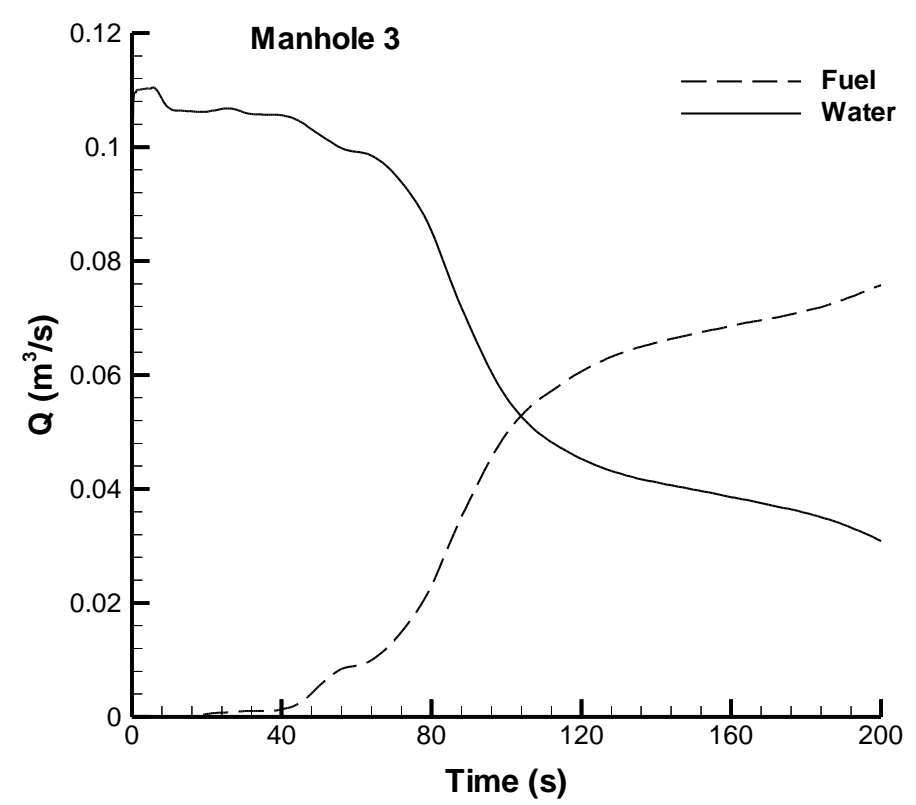

Figure 7.11 - Flow rates of fuel and water through manhole 3.

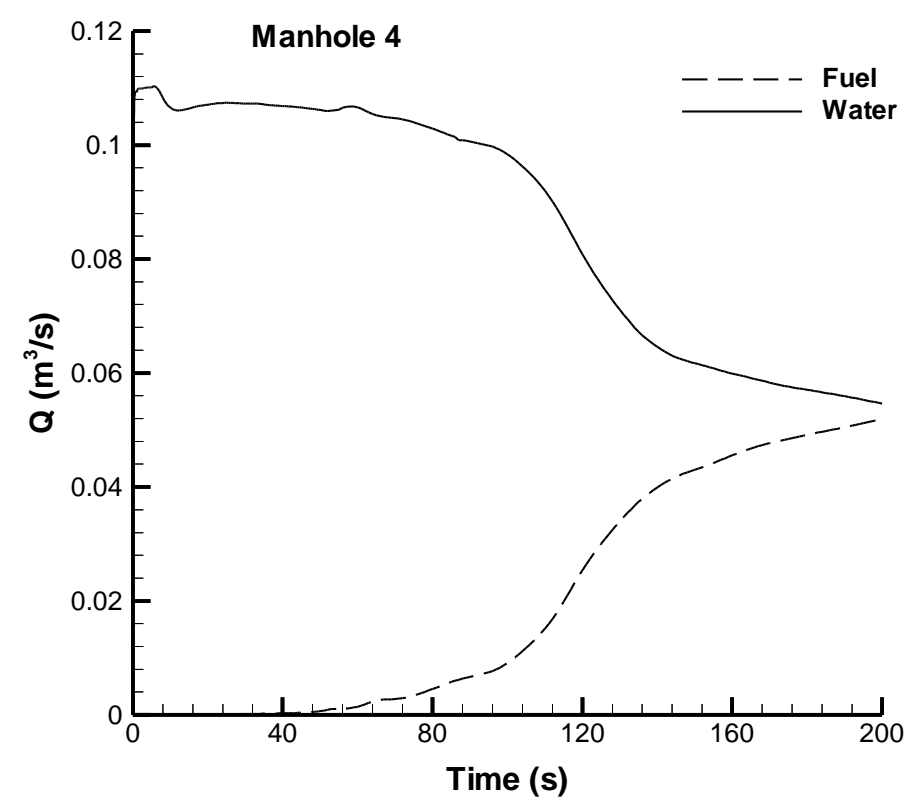

Figure 7.12 - Flow rates of fuel and water through manhole 4. 
To show the evolution of the fuel into the different compartments and to show the mixed interface, volume fraction profiles at 100.0, and 200.0 seconds are shown in Figure 7.13 through Figure 7.18. Figures 7.13 and 7.14 show longitudinal profiles, where the inlet and outlet blocks are indicated by white rectangles. In Figure 7.14 at 200.0 seconds the fuel is close to reaching the outlet. Since 200.0 seconds is less than half of the flow through time as computed from the tank volume and the inlet flow rate, the re-fueling efficiency is very poor for the present geometry. Here the re-fueling efficiency is defined as the time it takes the fuel to reach the outlet divided by the flow through time based on the total volume of the CFBT. A perfect re-fueling would require the entire flow through time and would fill the entire volume of the tank before the fuel reached the outlet.

Another observation that can be made from Figure 7.14 is that the compartments on the left side of the longitudinal manholes in the streamwise direction tend to fill earlier than the compartments on the right side. This should be expected considering that it is easier for the flow to spill through the large rectangular openings than for the flow to go through the transverse manholes. The capacity to establish a preferred path of flow based on the symmetry or lack thereof in the internal compartments is an important factor that should be considered in any re-design efforts since it will highly influence the mixing and refueling efficiency. 


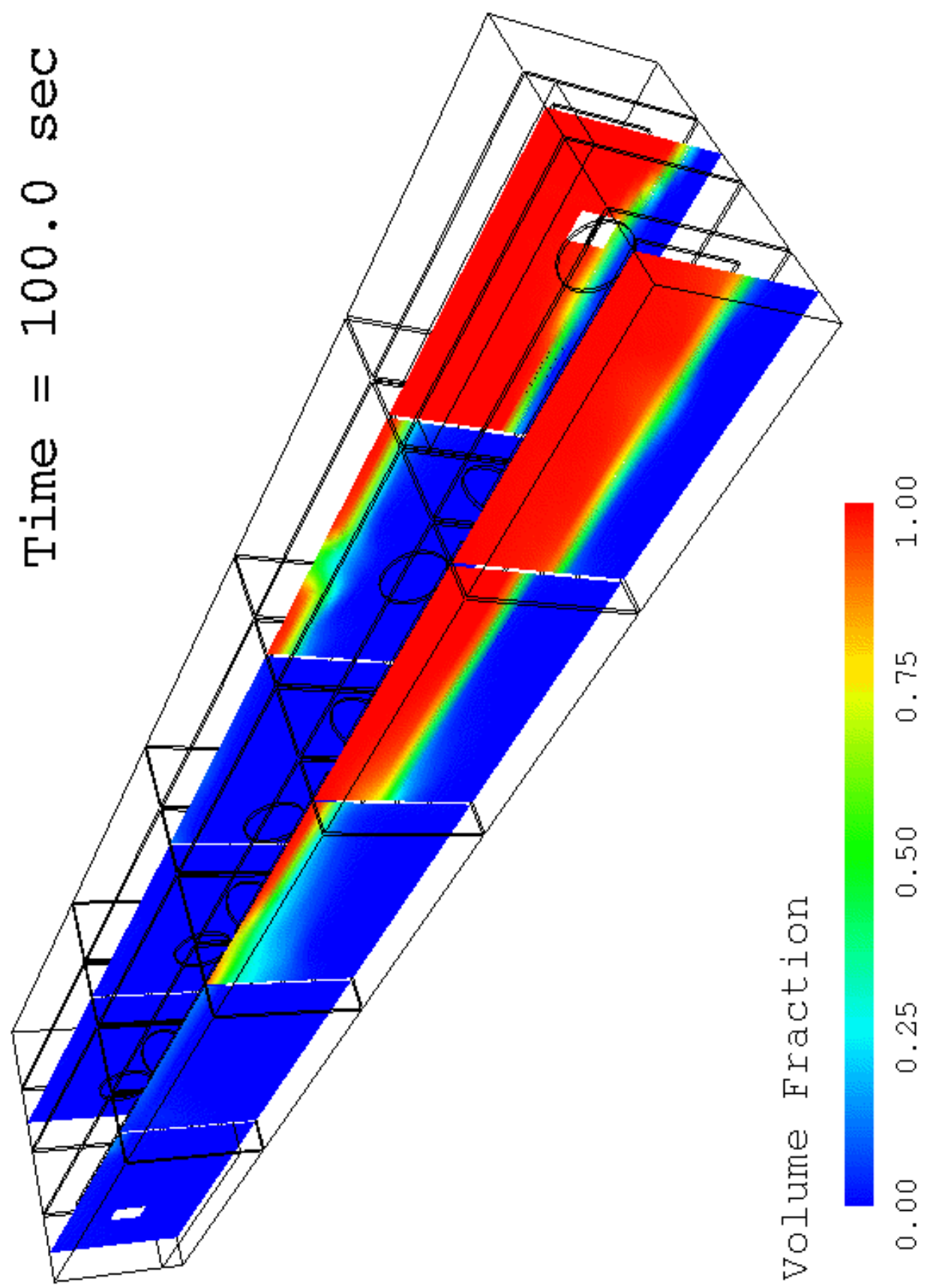

Figure 7.13 - Longitudinal volume fraction profiles in rows 1 and 3 after 100.0 seconds. 


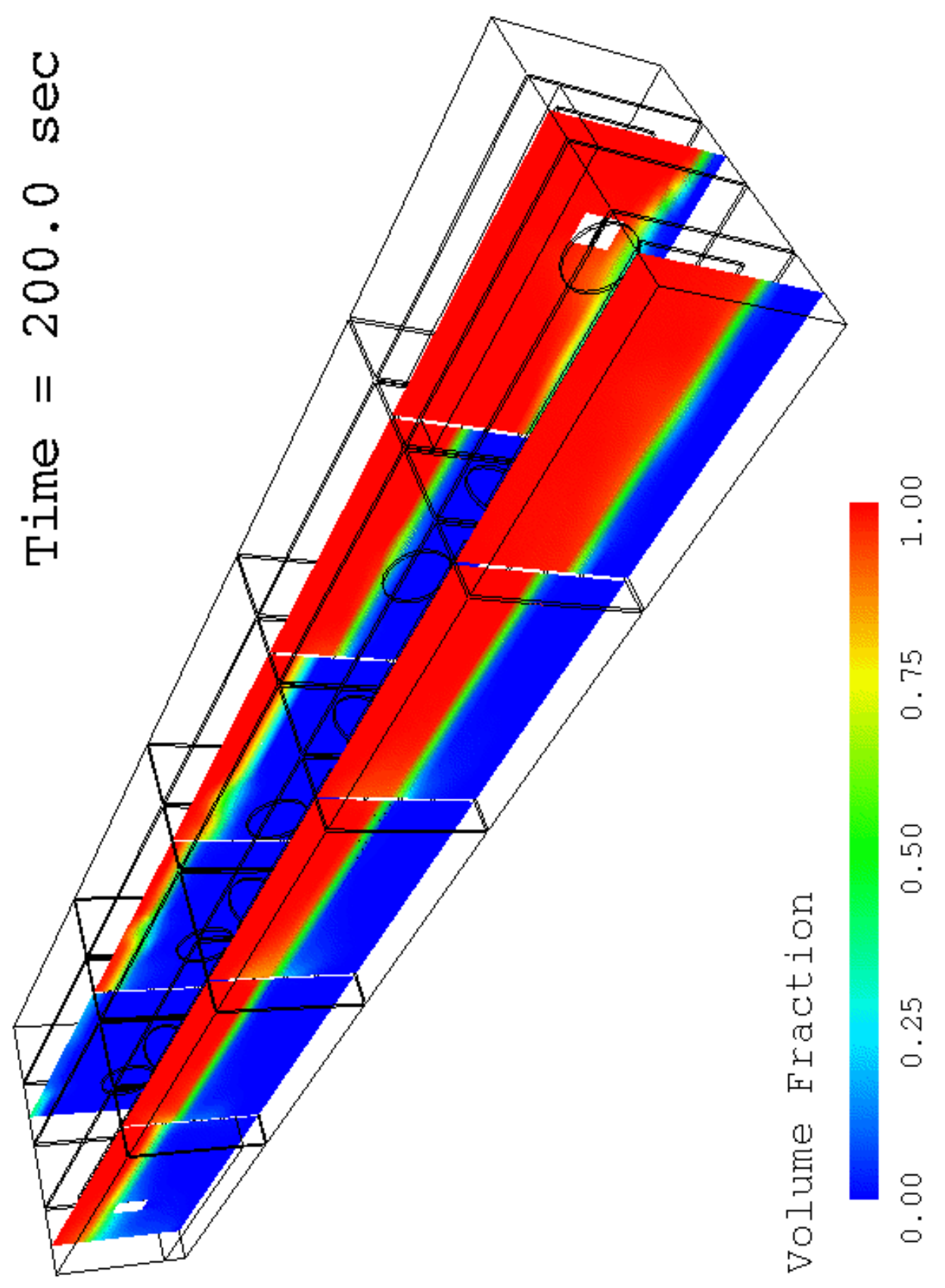

Figure 7.14 - Longitudinal volume fraction profiles in rows 1 and 3 after 200.0 seconds. 
The presence of water hideout, where water has been trapped inside various compartments, is obvious from Figure 7.13 and 7.14. It should be mentioned here that the limberholes would diminish the water hideout to a certain extent by allowing the water to flow out of the bottom of a compartment. This would not necessarily solve the problem of how quickly the fuel reaches the outlet, since the fuel will still travel the path of least resistance and would still prefer to flow through the center row of manholes. The present simulations can be viewed as being what would happen in the limit as the area of the limberholes went to zero.

Figures 7.15 and 7.16 show longitudinal profiles, where the flow through the main section of manholes can be seen at 100.0 and 200.0 seconds. In Figure 7.15 several stages of buoyant flow events can be seen along the longitudinal manholes. Figures 7.17 and 7.18 show several volume fraction profiles along transverse planes which indicate the manner in which the fuel spills into the transverse compartments. The water hideout is also obvious from the transverse profiles, especially in the compartments near the inlet. In Figure 7.18 at least two compartments on the far side of the CFBT, opposite to the outlet, are totally unfilled. 


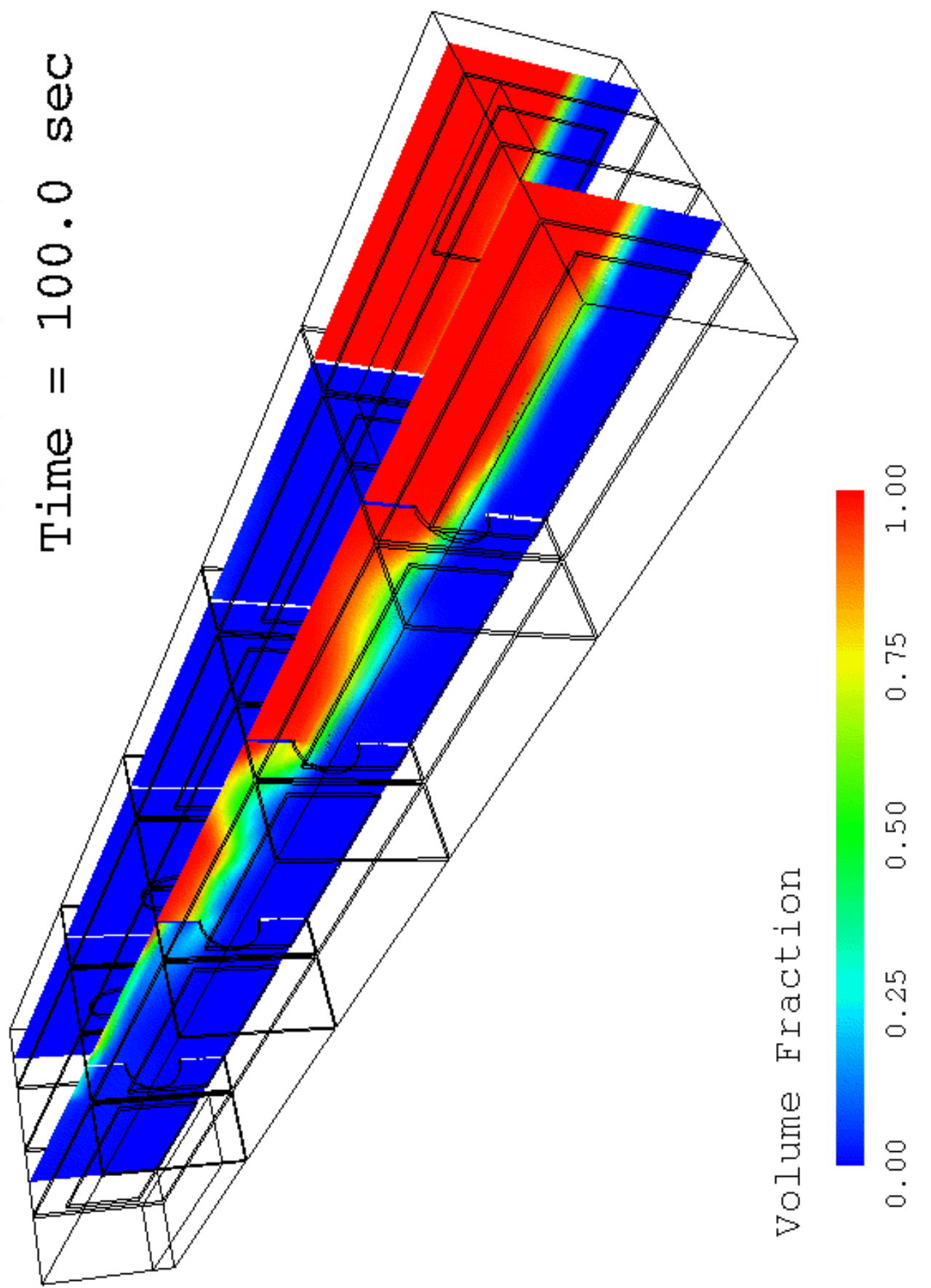

Figure 7.15 - Longitudinal volume fraction profiles in rows 2 and 4 after 100.0 seconds. 


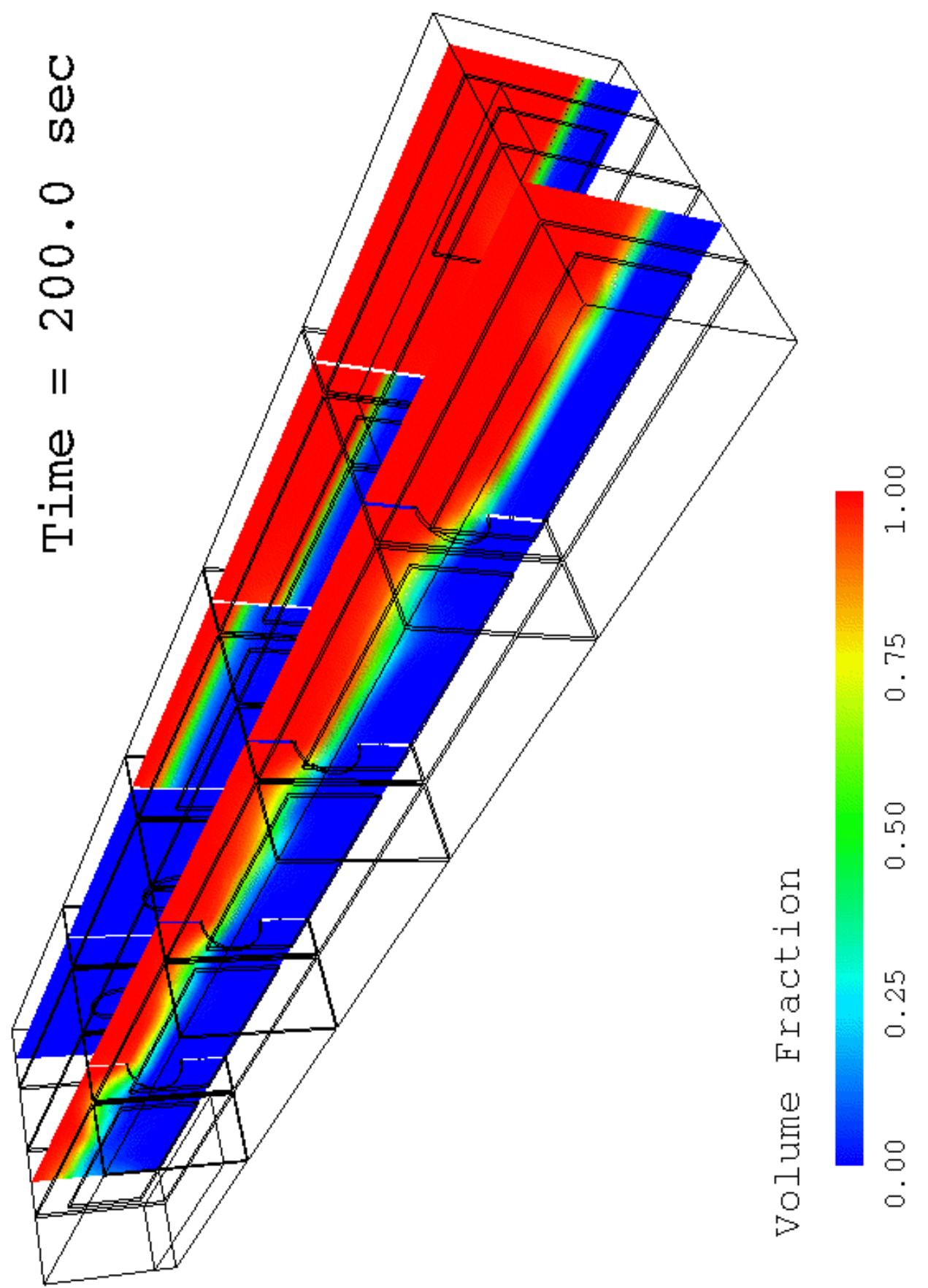

Figure 7.16 - Longitudinal volume fraction profiles in rows 2 and 4 after 200.0 seconds. 


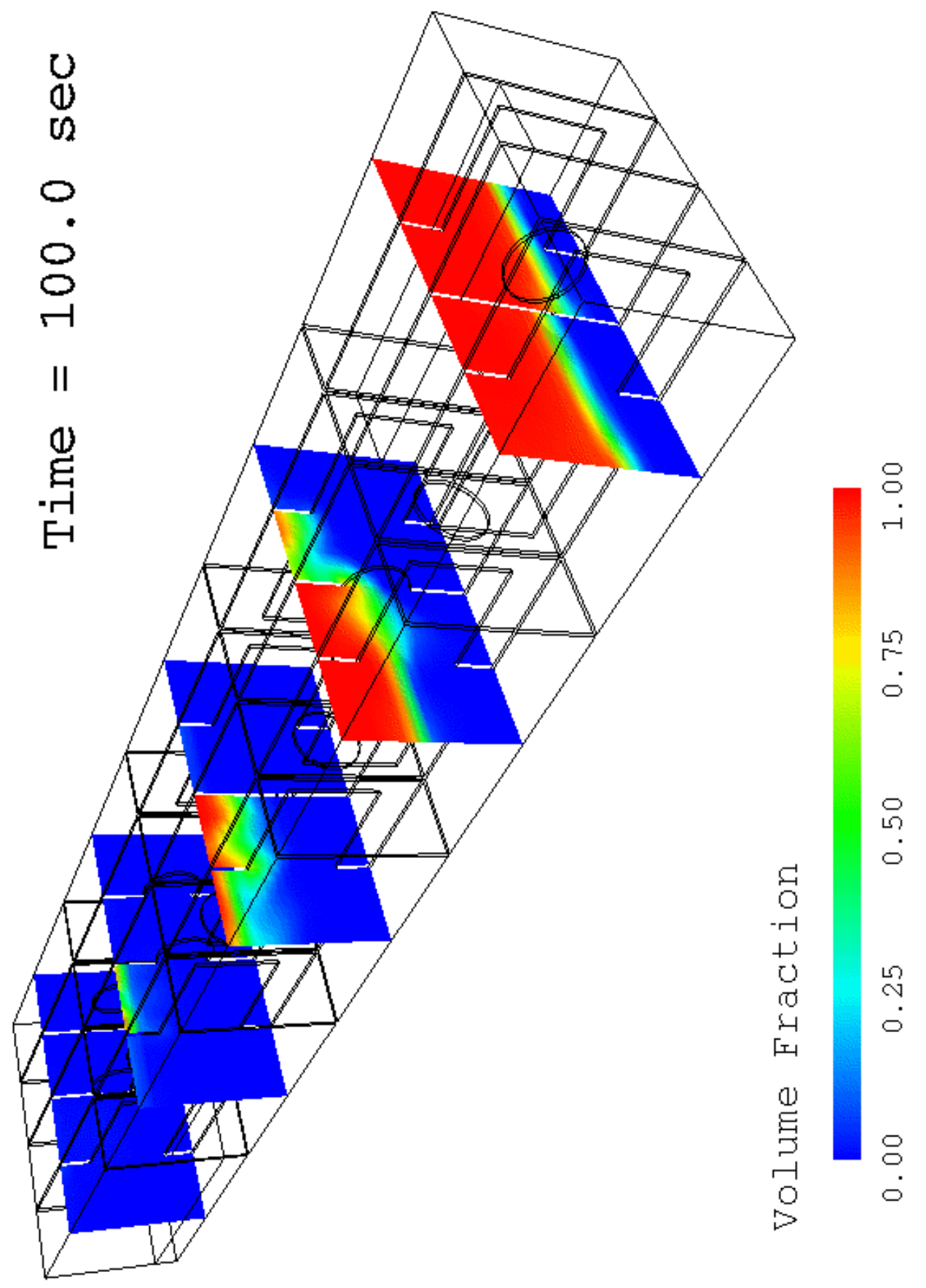

Figure 7.17 - Transverse volume fraction profiles after 100.0 seconds. 


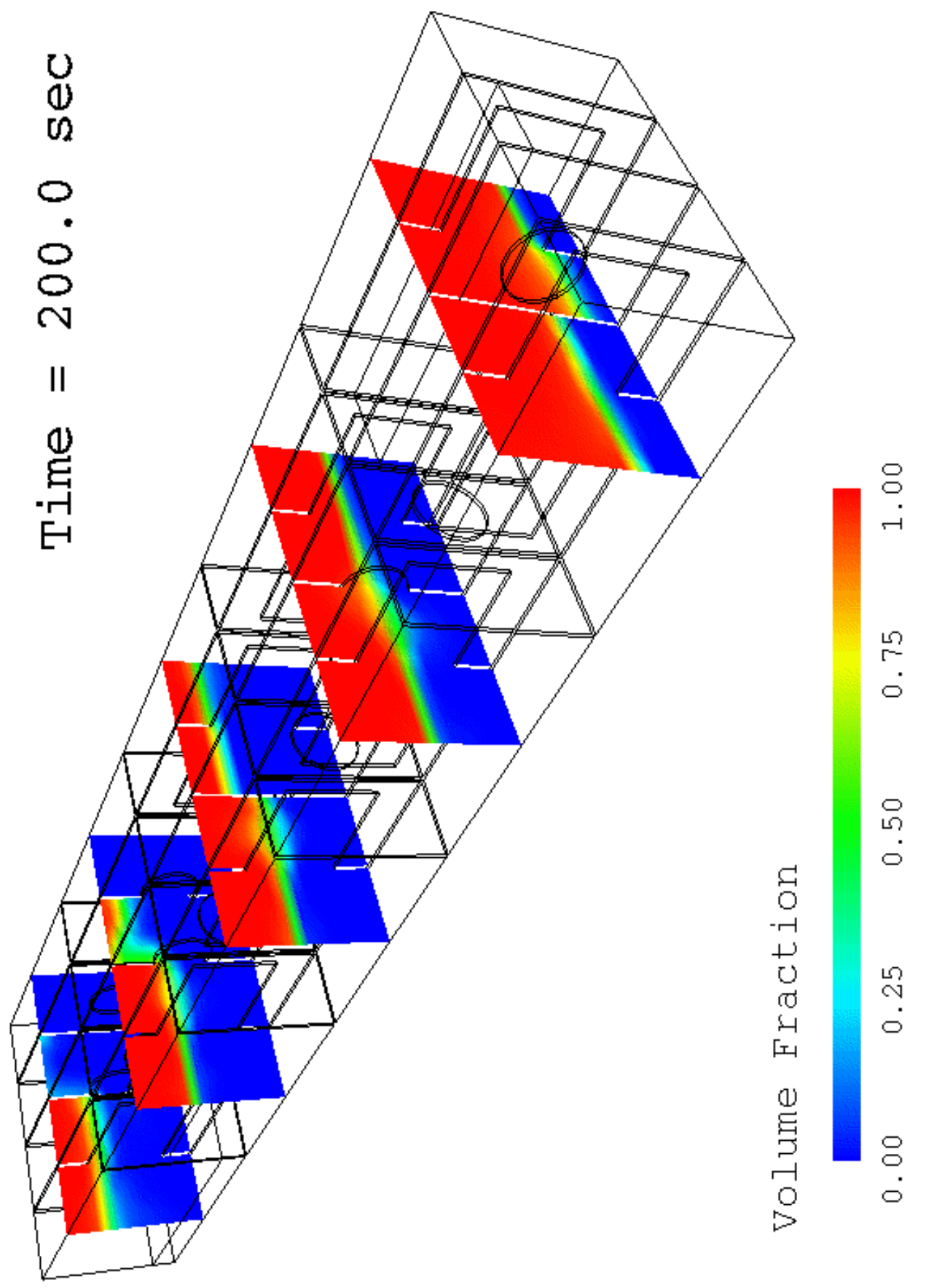

Figure 7.18 - Transverse volume fraction profiles after 200.0 seconds. 
Overall, the simulations conducted using SFST2 have demonstrated the applicability of single fluid scalar transport models to a full scale CFBT where complicated mixing situations occur and where relative velocity effects and computational efficiency are important. The model exhibited good convergence and captures many of the relevant physics that are important in modeling immiscible fluids. The transient mixing process showed that buoyant flow events that occur around manholes and internal openings are important regions where a significant amount of mixing can occur. The re-fueling efficiency was also shown to be deficient in the present geometry, since the fuel reaches the outlet at approximately half of the flow-through time. Water hideout was also demonstrated and is an obvious concern for future designs. 


\subsection{CONCLUSIONS}

\subsection{Summary}

Homogeneous, density stratified, shearing flows involving miscible fluids were simulated using the commercial code CFX 4.1. Using a single fluid scalar transport model and the $k-\varepsilon$ model with additional buoyancy production and dissipation terms, solutions were obtained for four cases that were investigated experimentally by Sullivan and List [28]. The simulations were compared with all of the relevant experimental parameters, and the majority of the predicted parameters were in qualitative agreement with their experimental counterparts. The results as predicted by the model indicate that the concentration boundary layers and individual layer Richardson numbers were possibly influenced by some of the boundary conditions, which had to be estimated for the simulations. Also, trends in the interfacial parameters were difficult to predict, presumably because of low Reynolds number effects that are not accounted for by the present form of the $k-\varepsilon$ model that was used. Overall, reasonably good trends were shown for the interfacial thickness, $\delta$, the mixed fluid thickness, $\delta_{\mathrm{M}}$, the mixed layer Richardson number, $\mathrm{Ri}_{\delta \mathrm{M}}$, and the dimensionless entrainment, $\mathrm{E}$. In considering the overall performance of the model, the majority of the parameters were well predicted in both trend and magnitude, when considered in light of the longitudinally averaged values. An interfacial thickness relationship given by $\delta / \mathrm{H} \sim\left(\mathrm{Ri}^{*}\right)^{-2.0}$ and an entrainment relationship given by $\mathrm{E} \sim\left(\mathrm{Ri}^{*}\right)^{-1.1}$ were predicted by the model and have been found to be in good agreement with experimental observations. 
For the simulations of the developing mixing layer involving diesel fuel and fresh water, time averaged, steady state solutions were obtained using a single fluid, scalar transport model that is based on a variant of Ishii's [16] drift flux model. The $k-\varepsilon$ turbulence model with extra terms to account for buoyancy effects was also used in these simulations. The ability to account for relative velocity effects in stratified, shearing flows was investigated, with the goal of quantitatively reproducing what is typically observed in experiments involving immiscible fluids.

Results from these simulations show a strong gradient Richardson number dependence. The gradient Richardson numbers in the simulations ranged from $\mathrm{Ri}_{\mathrm{G}}=0.05$ to $\mathrm{Ri}_{\mathrm{G}}=$ 0.25. Overall, the simulations performed with SFST2, the SFST model that includes effects of the slip velocity, gave reasonable predictions for the overall flow field and the parameters related to the mixed layer thickness. The predicted flow field was confirmed to qualitatively represent the actual flow field that was observed at the shear flow facility at Johns Hopkins University [38]. This comparison was done using several instantaneous profiles of the mixed interface at various locations along the shear layer. Average sizes for the droplets and the influence of the zone of re-circulation near the fuel inlet diffuser were also considered. The manner in which $\delta$ and $\delta_{\mathrm{M}}$ first increase and then decrease seems to agree with what has been observed in the experiments at Johns Hopkins University [38].

A three-dimensional, transient simulation of a typical compensated fuel/ballast tank was performed to demonstrate that SFST2 can be used to model complicated flow scenarios 
and study different aspects of the flow inside a CFBT. The simulations conducted using SFST2 demonstrated the ability of single fluid scalar transport models to model complicated mixing situations, where relative velocity effects and computational efficiency are important. The model exhibited good convergence and captured many of the relevant physics that are important in modeling immiscible fluids. The transient mixing process showed that buoyant flow events around manholes and internal openings are important areas where a significant amount of mixing can occur. The re-fueling efficiency was also shown to be deficient in the present geometry, since the fuel reaches the outlet in approximately half of the flow-through time. Water hideout was also demonstrated and is an obvious concern for future designs.

\subsection{Recommendations for Future Work}

For the simulations of the experiments performed by Sullivan and List [28], the following recommendations for further study are suggested. First, it would be beneficial to perform the simulations using a low Reynolds number $k-\varepsilon$ model. This would aid in determining if the typical low Reynolds number modifications would improve the predictions, especially in relation to the interfacial parameters. In conjunction with running the low Reynolds number model, refining the grid near the interface may also improve the predictions. Recognizing that inlet effects may also have been important, some improvement on the information at the boundaries from the experimenters could also be used to improve the predictions or at least eliminate boundary conditions as a source of error. Finally, an algebraic stress or second moment closure turbulence model should be tried to see if any significant improvement is gained. 
The simulations of the shear flow at Johns Hopkins should be compared with qualitative experimental data. Specifically, time averaged contours of the volume fractions and velocity vectors would be valuable for testing the model's ability to predict parameters related to entrainment and mixing. It would be advantageous to perform the simulations with a dynamic model which predicts the droplet size locally. This would allow better prediction of the slip velocity, and indicate where small droplets are being formed. It is also recommended that multiple particle effects, droplet distortion, and more extensive drag laws be considered in the formulations used for the slip velocity. Such formulations could be calibrated with results from the experiments at Johns Hopkins University.

Future simulations of the full scale CFBT should included the limberholes and the slope in the tank floor in the CFX geometry. It would be desirable to perform the simulations with a dynamic model that would predict the droplet size locally. This would provide insight into where small droplets are being formed in the re-fueling process. Comparison with experimental results should also be performed to validate the computational model. The degree to which the current accuracy of the model permits the model to be used as a design tool should be given serious consideration in light of any experimental data that is obtained. Finally, considering the fact that in some areas of the compensated fuel/ballast tanks the flow is semi-quiescent, the drift flux terms in the momentum equations may be of some importance. At the very least the drift flux terms will act to add a term in the momentum equations where the liquids are separating and the only realistic motion is buoyancy induced. 


\section{BIBLIOGRAPHY}

1. Atsavapranee, P. and Gharib, M. 1997. "Structures in stratified plane mixing layers and the effects of cross-shear." Journal of Fluid Mechanics. 342: 53-86.

2. Burns, A. 1997. CFX-F3D, Version 4.1 Advanced Course Notes: Introduction to Multiphase Modeling. AEA Technology, Bethel Park, PA.

3. Chang, P., Percival, S., and Hill, B. 1996. "Transient, two fluid flow through two compartments of a compensated fuel-ballast tank." Proceedings of AEA Technology. The Third CFX International Users Conference, Chesham, Buckinghamshire, England, UK.

4. Chang, P., Hill, B., Busby, M., and Bruchman, D. 1997. “CFD Simulations of Baseline and Modified Configurations of DDG 51 Compensated Fuel/Ballast Tank 5-300-2-F. Hydromechanics Directorate, Research and Development Report, CRDKNSWC/HD-1370-02.

5. Chang, P., Hill, B., Busby, M., and Bruchman, D. 1998. "CFD and Structural Analyses of Baseline and Modified Configurations of DDG 51 Compensated Fuel/Ballast Tank 5-300-2-F. Hydromechanics Directorate, Research and Development Report, CRDKNSWC/HD-1370-02.

6. CFX-F3D Flow Solver User Guide, Version 4.1. 1995. Computational Fluid Dynamics Services, Building 8.19, Harwell Laboratory, Oxfordshire OX11, ORA UK, October 1995.

7. Coulaloglou, C. and Tavlarides, L. 1977. "Description of Interaction Processes in Agitated Liquid-Liquid Dispersions.” Chemical Engineering Sciences. 32: 1289-1297.

8. Deardorff, J. and Willis, G. 1982. "Dependence of mixed-layer entrainment on shear stress and velocity jump." Journal of Fluid Mechanics. 115: 123-149.

9. Drew, D. 1983. "Mathematical Modeling of Two-Phase Flow." Annual Review of Fluid Mechanics. 15: 261-291.

10. Fernando, H. 1991. "Turbulent Mixing in Stratified Fluids." Annual Review of Fluid Mechanics. 23: 455-493.

11. Ferziger, J. and Peric, M. 1996. Computational Methods for Fluid Dynamics. Berlin: Springer. 
12. Fletcher, D. and Witt P. 1996. "Numerical studies of multiphase mixing with application to some small-scale experiments." Nuclear Engineering and Design. 166: $135-145$.

13. Hayworth, C. and Treybal, R. 1950. "Drop Formation in Two-Liquid-Phase Systems." Industrial and Engineering Chemistry. 6: 1174-1181.

14. Hetsroni, G. 1982. Handbook of Multiphase Systems. New York: Hemisphere Publishing Company.

15. Holt, S., Koseff, J. and Ferziger, J. 1992. "A numerical study of the evolution and structure of homogeneous stably stratified sheared turbulence." Journal of Fluid Mechanics. 237: 499-539.

16. Ishii, M. 1975. Thermo-Fluid Dynamic Theory of Two-Phase Flow. Paris: Eyrolles.

17. Ishii, M. 1977. "One-Dimensional Drift-Flux Model and Constitutive Equations for Relative Motion between Phases in Various Two-Phase Flow Regimes." Argonne National Laboratory Report, ANL-77-47.

18. Ishii, M. and Zuber, N. 1979. "Drag Coefficient and Relative Velocity in Bubbly, Droplet or Particulate Flows.” AIChE Journal. 25: 843-855.

19. Jacobitz, F., Sarkar, S. and Van Atta, C. 1997. "Direct numerical simulations of the turbulence evolution in a uniformly sheared and stably stratified flow." Journal of Fluid Mechanics. 342: 231-261.

20. Katz, J. 1998. Johns Hopkins University, Baltimore, MD. Personal contact.

21. Kranenburg, C. 1996. "On gradient transport turbulence models for stably stratified shear flow." Dynamics of Atmospheres and Oceans. 23: 205-215.

22. Miles, J. 1984. "Richardson number criterion for the stability of stratified shear flow.” Phys. Fluids. 29: 3470-3471.

23. Moore, M. and Long, R. 1971. "An Experimental Investigation of Turbulent Stratified Shearing Flow." Journal of Fluid Mechanics. 49: 635-655.

24. Narimousa, S. and Fernando, H. 1987. "On the sheared density interface of an entraining stratified fluid." Journal of Fluid Mechanics. 174: 1-22.

25. Rodi, W. 1980. "Turbulence Models and Their Application in Hydraulics - A Stateof-the-Art Review." An IAHR (International Association of Hydraulic Research) Publication, Delft, The Netherlands. 
26. Ruddick, B., McDougall, T. and Turner, J. 1989. "The formation of layers in a uniformly stirred density gradient.” Deep-Sea Res. 36: 597-609.

27. Sullivan, G. 1992. "On Mixing and Transport at a Sheared Density Interface," Ph.D. Dissertation. California Institute of Technology.

28. Sullivan, G. and List, J. 1994. "On mixing and transport at a sheared density interface." Journal of Fluid Mechanics. 273: 213-239.

29. Tennekes H. and Lumley J. 1972. A First Course in Turbulence. Cambridge: Massachusetts Institute of Technology Press.

30. Townsend, A. 1958. "The effects of radiative transfer on turbulent flow of a stratified fluid." Journal of Fluid Mechanics. 4: 361-375.

31. Townsend, A. 1976. The Structure of Turbulent Shear Flow. Second Edition. London: Cambridge University Press.

32. Tsouris C. and Tavlarides L. 1994. "Breakage and Coalescence Models for Drops in Turbulent Dispersions." AIChE Journal. 40: 395-406.

33. Turner, J. 1973. Buoyancy Effects in Fluids. London: Cambridge University Press.

34. Turner, J. and Ellison, T. 1959. "Turbulent entrainment in stratified flows." Journal of Fluid Mechanics. 28: 423-448.

35. Wallis, G. 1969. One-dimensional Two-phase Flow. New York: McGraw-Hill.

36. White, F. 1991. Viscous Fluid Flow. New York: McGraw-Hill.

37. Wilcox, D. 1993. Turbulence Modeling for CFD. Glendale: Griffin Printing.

38. Wu, X., Katz, J. 1998. "On the Flow Structure and Mixing Phenomena In a Water-Fuel Stratified Shear Layer." in preparation. 


\section{APPENDIX A: DERIVATION OF EQUATIONS FOR SFST MODEL}

\section{A.1 Model Assumptions, Definitions of Mixture Properties}

\section{Model Assumptions}

1. The flow is isothermal.

2. Neither phase is experiencing any mass production or destruction.

3. Both phases are incompressible in that $\rho_{\alpha}$ and $\rho_{\beta}$ are constants.

4. Both phases share the same pressure field (i.e. $p_{\alpha}=p_{\beta}$ ).

5. Both phases share the same turbulent stresses (i.e. $\tau_{\alpha}=\tau_{\beta}$ ).

6. The fluctuations in the mixture density or $\rho_{m}^{\prime} / \rho_{m}$ are negligible.

\section{Model Variables and Nomenclature}

$\begin{array}{ll}u & \text { Velocity } \\ \rho & \text { Density } \\ \mu & \text { Dynamic viscosity }(\mathrm{kg} / \mathrm{ms}) \\ \mathrm{r} & \text { Volume fraction of phase }\left(\mathrm{i} . \mathrm{e} . \mathrm{r}_{\alpha}=\mathrm{Vol}_{\alpha} / \mathrm{Vol}\right) \\ \mathrm{p} & \text { Pressure } \\ \alpha & \text { subscript denotes phase alpha } \\ \beta & \text { subscript denotes phase beta } \\ \sim & \text { tilda denotes instantaneous quantity }\left(\text { i.e. } \tilde{\phi}=\left(\phi+\phi^{\prime}\right)\right)\end{array}$

\section{Mixture Definitions}

1. Mixture density:

$$
\rho_{\mathrm{m}}=\rho_{\alpha} \mathrm{r}_{\alpha}+\rho_{\beta} \mathrm{r}_{\beta}
$$

2. Mixture viscosity:

$$
\mu_{\mathrm{m}}=\mu_{\alpha} \mathrm{r}_{\alpha}+\mu_{\beta} \mathrm{r}_{\beta}
$$

3. Mixture velocity:

$$
u_{\mathrm{m}}=\frac{\left(\mathrm{u}_{\alpha} \mathrm{r}_{\alpha} \rho_{\alpha}+\mathrm{u}_{\beta} \mathrm{r}_{\beta} \rho_{\beta}\right)}{\rho_{\mathrm{m}}}
$$

4. Slip velocity:

$$
\mathrm{u}_{\mathrm{s}}=\left(\mathrm{u}_{\alpha}-\mathrm{u}_{\beta}\right)
$$




\section{A.2 Reynolds Averaged Continuity Equation for SFST Model}

Given the instantaneous mixture density $\widetilde{\rho}_{\mathrm{m}}=\rho_{\alpha} \widetilde{\mathrm{r}}_{\alpha}+\rho_{\beta} \widetilde{\widetilde{\beta}}_{\beta}$, these could be re-written using time averaged and mean quantities as

$$
\tilde{\rho}_{\mathrm{m}}=\rho_{\alpha}\left(\mathrm{r}_{\alpha}+\mathrm{r}_{\alpha}^{\prime}\right)+\rho_{\beta}\left(\mathrm{r}_{\beta}+\mathrm{r}_{\beta}^{\prime}\right)
$$

it is easily shown that

$$
\rho_{\mathrm{m}}=\rho_{\alpha} \mathrm{r}_{\alpha}+\rho_{\beta} \mathrm{r}_{\beta} \quad \text { and } \quad \rho_{\mathrm{m}}^{\prime}=\rho_{\alpha} \mathrm{r}_{\alpha}^{\prime}+\rho_{\beta} \mathrm{r}_{\beta}^{\prime}
$$

and that

$$
\frac{\rho_{m}^{\prime}}{\rho_{m}}=\frac{\rho_{\alpha} r_{\alpha}^{\prime}+\rho_{\beta} r_{\beta}^{\prime}}{\rho_{\alpha} r_{\alpha}+\rho_{\beta} r_{\beta}}=\frac{\rho_{\alpha} r_{\alpha}^{\prime}+\rho_{\beta} r_{\beta}^{\prime}}{\rho_{\alpha} r_{\alpha}-\rho_{\beta} r_{\alpha}+\rho_{\beta}}
$$

Using the property that $\mathrm{r}_{\alpha}^{\prime}=-\mathrm{r}_{\beta}^{\prime}$ and that $\mathrm{r}_{\alpha}+\mathrm{r}_{\beta}=1.0$, Eq. (A.2.3) may be re-written as

$$
\frac{\rho_{\mathrm{m}}^{\prime}}{\rho_{\mathrm{m}}}=\frac{-\left(\rho_{\beta}-\rho_{\alpha}\right) \mathrm{r}_{\alpha}^{\prime}}{\rho_{\beta}-\left(\rho_{\beta}-\rho_{\alpha}\right) \mathrm{r}_{\alpha}}=\frac{\Delta \rho \mathrm{r}_{\alpha}^{\prime}}{\Delta \rho r_{\alpha}-\rho_{\beta}}
$$

The limiting cases of Eq. (A.2.4) can be considered to investigate the ratio of the fluctuating to mean mixture density. Given that $\mathrm{r}_{\alpha}+\mathrm{r}_{\alpha}^{\prime} \leq 1.0$ since the volume fractions must sum to one

$$
\begin{array}{ll}
\text { if } \mathrm{r}_{\alpha}=1.0 \text { then } \mathrm{r}_{\alpha}^{\prime}=0.0 \text { and } & \left|\frac{\rho_{\mathrm{m}}^{\prime}}{\rho_{\mathrm{m}}}\right|=0.0 \\
\text { if } \mathrm{r}_{\alpha}^{\prime}=1.0 \text { then } \mathrm{r}_{\alpha}=0.0 \text { and } & \left|\frac{\rho_{\mathrm{m}}^{\prime}}{\rho_{\mathrm{m}}}\right|=\left|\frac{\Delta \rho}{\rho_{\beta}}\right|<1.0
\end{array}
$$

Hence, for cases where $\Delta \rho$ is small compared to either of the phase densities, the density fluctuations may be neglected when normalized by the mixture density. It should be noted that $r_{\alpha}^{\prime} / r_{\alpha}$ cannot be neglected since this term may attain significant magnitudes. With the mixture density fluctuations assumed negligible, the next task is the derivation of the time averaged mixture continuity equation. The beginning of this derivation is the pure unmixed continuity equations, as given by Ishii, for phase $\alpha$

$$
\frac{\partial \widetilde{\mathrm{r}}_{\alpha} \rho_{\alpha}}{\partial \mathrm{t}}+\frac{\partial \widetilde{\mathrm{r}}_{\alpha} \rho_{\alpha} \widetilde{\mathrm{u}}_{\alpha, \mathrm{i}}}{\partial \mathrm{x}_{\mathrm{i}}}=0.0
$$


and phase $\beta$

$$
\frac{\partial \widetilde{\mathrm{r}}_{\beta} \rho_{\beta}}{\partial \mathrm{t}}+\frac{\partial \widetilde{\mathrm{r}}_{\beta} \rho_{\beta} \widetilde{\mathrm{u}}_{\beta, \mathrm{i}}}{\partial \mathrm{x}_{\mathrm{i}}}=0.0
$$

Adding Eq. (A.2.7) and Eq. (A.2.8) gives

$$
\frac{\partial \widetilde{\rho}_{m}}{\partial t}+\frac{\partial \widetilde{\rho}_{m} \widetilde{u}_{m, i}}{\partial x_{i}}=0.0
$$

which is re-written as

$$
\frac{\partial}{\partial t}\left[\rho_{m}\left(1+\frac{\rho_{m}^{\prime}}{\rho_{m}}\right)\right]+\frac{\partial}{\partial x_{i}}\left[\rho_{m}\left(1+\frac{\rho_{m}^{\prime}}{\rho_{m}}\right)\left(u_{m, i}+u_{m, i}^{\prime}\right)\right]=0.0
$$

which, upon neglecting the terms involving density fluctuations and averaging gives

$$
\frac{\partial \rho_{m}}{\partial t}+\frac{\partial \rho_{m} u_{m, i}}{\partial x_{i}}=0.0
$$

for the SFST model, where all variables are understood to be time averaged. A similar argument may be made to neglect the density fluctuations in the momentum equations. 


\section{A.3 Incompressible law for Mixture Velocity}

Regarding the use of a generic scalar transport equation for the volume fraction equation, it must hold that the divergence of the mixture velocity given by

$$
\frac{\partial \mathrm{u}_{\mathrm{m}, \mathrm{i}}}{\partial \mathrm{x}_{\mathrm{i}}}=\frac{\partial}{\partial \mathrm{x}_{\mathrm{i}}}\left(\frac{\mathrm{u}_{\alpha} \mathrm{r}_{\alpha} \rho_{\alpha}+\mathrm{u}_{\beta} \mathrm{r}_{\beta} \rho_{\beta}}{\rho_{\mathrm{m}}}\right)
$$

is zero, where $\rho_{\mathrm{m}}=\mathrm{r}_{\alpha} \rho_{\alpha}+\mathrm{r}_{\beta} \rho_{\beta}$. This proof can be accomplished by first considering the general relations given by the individual phase continuity equations. As the two individual phases are regarded as incompressible since $\rho_{\alpha}$ and $\rho_{\beta}$ are constants, the two relations given by

$$
\frac{\partial \tilde{\mathrm{u}}_{\alpha, \mathrm{i}}}{\partial \mathrm{x}_{\mathrm{i}}}=0.0 \quad \text { and } \quad \frac{\partial \tilde{\mathrm{u}}_{\beta, \mathrm{i}}}{\partial \mathrm{x}_{\mathrm{i}}}=0.0
$$

hold true. These relations further imply that

$$
\frac{\partial\left(\tilde{\mathrm{r}}_{\alpha}\right)}{\partial \mathrm{t}}+\tilde{\mathrm{u}}_{\alpha, \mathrm{i}} \frac{\partial}{\partial \mathrm{x}_{\mathrm{i}}}\left(\widetilde{\mathrm{r}}_{\alpha}\right)=0.0 \quad \text { and } \quad \frac{\partial\left(\tilde{\mathrm{r}}_{\beta}\right)}{\partial \mathrm{t}}+\tilde{\mathrm{u}}_{\beta, \mathrm{i}} \frac{\partial}{\partial \mathrm{x}_{\mathrm{i}}}\left(\widetilde{\mathrm{r}}_{\beta}\right)=0.0
$$

by use of the individual phase continuity equations. Multiplying Eq. (A.3.3a) by $\rho_{\alpha}$ and Eq. (A.3.3b) by $\rho_{\beta}$ and then adding them gives

$$
\frac{\partial\left(\rho_{\alpha} \widetilde{\mathrm{r}}_{\alpha}+\rho_{\beta} \widetilde{\mathrm{r}}_{\beta}\right)}{\partial \mathrm{t}}+\frac{\widetilde{\rho}_{\mathrm{m}} \tilde{\mathrm{u}}_{\alpha, \mathrm{i}}}{\tilde{\rho}_{\mathrm{m}}} \frac{\partial\left(\rho_{\alpha} \widetilde{\mathrm{r}}_{\alpha}\right)}{\partial \mathrm{x}_{\mathrm{i}}}+\frac{\widetilde{\rho}_{\mathrm{m}} \widetilde{\mathrm{u}}_{\beta, \mathrm{i}}}{\tilde{\rho}_{\mathrm{m}}} \frac{\partial\left(\rho_{\beta} \widetilde{\mathrm{r}}_{\beta}\right)}{\partial \mathrm{x}_{\mathrm{i}}}=0.0
$$

which may be written as

$$
\begin{array}{r}
\frac{\partial\left(\tilde{\rho}_{m}\right)}{\partial t}+\frac{\tilde{\rho}_{m} \tilde{u}_{\alpha, i}}{\tilde{\rho}_{m}} \frac{\partial\left(\rho_{\alpha} \tilde{r}_{\alpha}\right)}{\partial x_{i}}+\frac{\tilde{\rho}_{m} \tilde{u}_{\beta, i}}{\tilde{\rho}_{m}} \frac{\partial\left(\rho_{\beta} \tilde{r}_{\beta}\right)}{\partial x_{i}}=0.0 \\
\frac{\partial \tilde{\rho}_{m}}{\partial t}+\left(\frac{\left(\rho_{\alpha} \tilde{r}_{\alpha}+\rho_{\beta} \tilde{r}_{\beta}\right) \tilde{u}_{\alpha, i}}{\tilde{\rho}_{m}}\right) \frac{\partial\left(\rho_{\alpha} \tilde{r}_{\alpha}\right)}{\partial x_{i}}+\left(\frac{\left(\rho_{\alpha} \tilde{r}_{\alpha}+\rho_{\beta} \tilde{r}_{\beta}\right) \tilde{u}_{\beta, i}}{\tilde{\rho}_{m}}\right) \frac{\partial\left(\rho_{\beta} \tilde{r}_{\beta}\right)}{\partial x_{i}}=0.0
\end{array}
$$


Adding and subtracting similar terms, we may write Eq. (A.3.6) as

$$
\begin{aligned}
& \frac{\partial \tilde{\rho}_{\mathrm{m}}}{\partial \mathrm{t}}+\left(\frac{\left(\rho_{\alpha} \tilde{\mathrm{r}}_{\alpha}+\rho_{\beta} \tilde{\mathrm{r}}_{\beta}\right) \tilde{\mathrm{u}}_{\alpha, \mathrm{i}}}{\tilde{\rho}_{\mathrm{m}}}\right) \frac{\partial\left(\rho_{\alpha} \tilde{\mathrm{r}}_{\alpha}\right)}{\partial \mathrm{x}_{\mathrm{i}}}+\left(\frac{\left(\rho_{\alpha} \tilde{\mathrm{r}}_{\alpha}+\rho_{\beta} \tilde{\mathrm{r}}_{\beta}\right) \tilde{\mathrm{u}}_{\beta, \mathrm{i}}}{\tilde{\rho}_{\mathrm{m}}}\right) \frac{\partial\left(\rho_{\beta} \tilde{\mathrm{r}}_{\beta}\right)}{\partial \mathrm{x}_{\mathrm{i}}}+ \\
& \left(\frac{\rho_{\beta} \tilde{\mathrm{r}}_{\beta} \tilde{\mathrm{u}}_{\beta, \mathrm{i}}}{\tilde{\rho}_{\mathrm{m}}}-\frac{\rho_{\beta} \tilde{\mathrm{r}}_{\beta} \tilde{\mathrm{u}}_{\beta, \mathrm{i}}}{\tilde{\rho}_{\mathrm{m}}}\right) \frac{\partial\left(\rho_{\alpha} \tilde{\mathrm{r}}_{\alpha}\right)}{\partial \mathrm{x}_{\mathrm{i}}}+\left(\frac{\rho_{\alpha} \tilde{\mathrm{r}}_{\alpha} \tilde{\mathrm{u}}_{\alpha, \mathrm{i}}}{\tilde{\rho}_{\mathrm{m}}}-\frac{\rho_{\alpha} \tilde{\mathrm{r}}_{\alpha} \tilde{\mathrm{u}}_{\alpha, \mathrm{i}}}{\tilde{\rho}_{\mathrm{m}}}\right) \frac{\partial\left(\rho_{\beta} \tilde{\mathrm{r}}_{\beta}\right)}{\partial \mathrm{x}_{\mathrm{i}}}=0.0
\end{aligned}
$$

which is re-written as

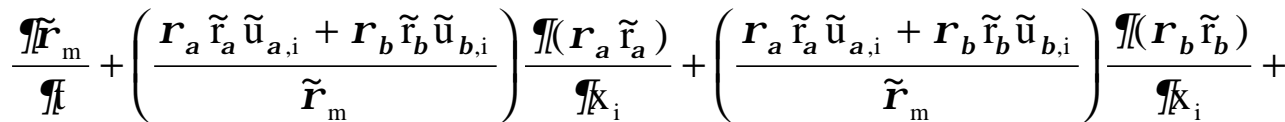

$$
\begin{aligned}
& \left(\frac{\rho_{\beta} \tilde{\mathrm{r}}_{\beta} \tilde{\mathrm{u}}_{\alpha, \mathrm{i}}}{\tilde{\rho}_{\mathrm{m}}}-\frac{\rho_{\beta} \tilde{\mathrm{r}}_{\beta} \tilde{\mathrm{u}}_{\beta, \mathrm{i}}}{\tilde{\rho}_{\mathrm{m}}}\right) \frac{\partial\left(\rho_{\alpha} \tilde{\mathrm{r}}_{\alpha}\right)}{\partial \mathrm{x}_{\mathrm{i}}}+\left(\frac{\rho_{\alpha} \tilde{\mathrm{r}}_{\alpha} \tilde{\mathrm{u}}_{\beta, \mathrm{i}}}{\tilde{\rho}_{\mathrm{m}}}-\frac{\rho_{\alpha} \tilde{\mathrm{r}}_{\alpha} \tilde{\mathrm{u}}_{\alpha, \mathrm{i}}}{\tilde{\rho}_{\mathrm{m}}}\right) \frac{\partial\left(\rho_{\beta} \tilde{\mathrm{r}}_{\beta}\right)}{\partial \mathrm{x}_{\mathrm{i}}}=0.0
\end{aligned}
$$

which by the definition of $\mathrm{u}_{\mathrm{m}}$, gives

$$
\frac{\partial \tilde{\rho}_{m}}{\partial t}+\widetilde{u}_{m, i} \frac{\partial \tilde{\rho}_{m}}{\partial x_{i}}+\frac{\rho_{\alpha} \rho_{\beta} \widetilde{r}_{\beta}}{\tilde{\rho}_{m}}\left(\widetilde{u}_{\alpha, i}-\widetilde{u}_{\beta, i}\right) \frac{\partial \widetilde{r}_{\alpha}}{\partial x_{i}}+\frac{\rho_{\alpha} \rho_{\beta} \widetilde{r}_{\alpha}}{\tilde{\rho}_{m}}\left(\widetilde{u}_{\beta, i}-\widetilde{u}_{\alpha, i}\right) \frac{\partial \widetilde{r}_{\beta}}{\partial x_{i}}=0.0
$$

Now realize that

$$
\frac{\partial \widetilde{r}_{\alpha}}{\partial x_{i}}=\frac{\partial\left(1-\tilde{r}_{\beta}\right)}{\partial x_{i}}=-\frac{\partial \tilde{r}_{\beta}}{\partial x_{i}} \quad \text { and } \quad \frac{\partial \widetilde{r}_{\beta}}{\partial x_{i}}=\frac{\partial\left(1-\widetilde{r}_{\alpha}\right)}{\partial x_{i}}=-\frac{\partial \widetilde{r}_{\alpha}}{\partial x_{i}}
$$

which allows Eq. (9) to be written as

$$
\frac{\partial \tilde{\rho}_{m}}{\partial t}+\widetilde{u}_{m, i} \frac{\partial \tilde{\rho}_{m}}{\partial x_{i}}+\frac{\rho_{\alpha} \rho_{\beta} \widetilde{r}_{\beta}}{\tilde{\rho}_{m}}\left(\widetilde{u}_{\alpha, i}-\widetilde{u}_{\beta, i}\right) \frac{\partial \widetilde{r}_{\alpha}}{\partial x_{i}}+\frac{\rho_{\alpha} \rho_{\beta} \widetilde{r}_{\alpha}}{\tilde{\rho}_{m}}\left(\widetilde{u}_{\alpha, i}-\widetilde{u}_{\beta, i}\right) \frac{\partial \widetilde{r}_{\alpha}}{\partial x_{i}}=0.0
$$

or

$$
\frac{\partial \tilde{\rho}_{m}}{\partial t}+\tilde{u}_{m, i} \frac{\partial \tilde{\rho}_{m}}{\partial x_{i}}+\frac{\rho_{\alpha} \rho_{\beta}}{\tilde{\rho}_{m}}\left(\tilde{u}_{\alpha, i}-\tilde{u}_{\beta, i}\right) \frac{\partial \tilde{r}_{\alpha}}{\partial x_{i}}\left(\tilde{r}_{\alpha}+\tilde{r}_{\beta}\right)=0.0
$$

Using the fact that the instantaneous volume fractions must sum to 1.0 gives

$$
\frac{\partial \widetilde{\rho}_{m}}{\partial t}+\tilde{u}_{m, i} \frac{\partial \tilde{\rho}_{m}}{\partial x_{i}}+\frac{\rho_{\alpha} \rho_{\beta}}{\tilde{\rho}_{m}}\left(\tilde{u}_{\alpha, i}-\tilde{u}_{\beta, i}\right) \frac{\partial \widetilde{r}_{\alpha}}{\partial x_{i}}=0.0
$$


and using the relation given in Eq. (A.3.10a) we have

$$
\frac{\partial \tilde{\rho}_{m}}{\partial t}+\tilde{u}_{m, i} \frac{\partial \tilde{\rho}_{m}}{\partial x_{i}}+\frac{\rho_{\alpha} \rho_{\beta}}{\tilde{\rho}_{m}}\left(\tilde{u}_{\alpha, i} \frac{\partial \widetilde{r}_{\alpha}}{\partial x_{i}}+\tilde{u}_{\beta, i} \frac{\partial \tilde{r}_{\beta}}{\partial x_{i}}\right)=0.0
$$

Adding and subtracting the time derivative of the volume fraction for phase $\alpha$ to the last term in Eq. (A.3.14) we have

$$
\frac{\partial \widetilde{\rho}_{m}}{\partial t}+\widetilde{u}_{m, i} \frac{\partial \tilde{\rho}_{m}}{\partial x_{i}}+\frac{\rho_{\alpha} \rho_{\beta}}{\tilde{\rho}_{m}}\left(\frac{\partial \widetilde{r}_{\alpha}}{\partial t}+\widetilde{u}_{\alpha, i} \frac{\partial \widetilde{r}_{\alpha}}{\partial x_{i}}-\frac{\partial \widetilde{r}_{\alpha}}{\partial t}+\widetilde{u}_{\beta, i} \frac{\partial \widetilde{r}_{\beta}}{\partial x_{i}}\right)=0.0
$$

which using the fact that

$$
\frac{\partial \tilde{\mathrm{r}}_{\alpha}}{\partial \mathrm{t}}=\frac{\partial\left(1-\widetilde{\mathrm{r}}_{\beta}\right)}{\partial \mathrm{t}}=-\frac{\partial \widetilde{\mathrm{r}}_{\beta}}{\partial \mathrm{t}} \quad \text { and } \quad \frac{\partial \widetilde{\mathrm{r}}_{\beta}}{\partial \mathrm{t}}=\frac{\partial\left(1-\widetilde{\mathrm{r}}_{\alpha}\right)}{\partial \mathrm{t}}=-\frac{\partial \widetilde{\mathrm{r}}_{\alpha}}{\partial \mathrm{t}}
$$

we can write

$$
\frac{\partial \tilde{\rho}_{\mathrm{m}}}{\partial \mathrm{t}}+\tilde{\mathrm{u}}_{\mathrm{m}, \mathrm{i}} \frac{\partial \tilde{\rho}_{\mathrm{m}}}{\partial \mathrm{x}_{\mathrm{i}}}+\frac{\rho_{\alpha} \rho_{\beta}}{\tilde{\rho}_{\mathrm{m}}}\left[\left(\frac{\partial \widetilde{\mathrm{r}}_{\alpha}}{\partial \mathrm{t}}+\widetilde{\mathrm{u}}_{\alpha, \mathrm{i}} \frac{\partial \widetilde{\mathrm{r}}_{\alpha}}{\partial \mathrm{x}_{\mathrm{i}}}\right)+\left(\frac{\partial \widetilde{\mathrm{r}}_{\beta}}{\partial \mathrm{t}}+\widetilde{\mathrm{u}}_{\beta, \mathrm{i}} \frac{\partial \widetilde{\mathrm{r}}_{\beta}}{\partial \mathrm{x}_{\mathrm{i}}}\right)\right]=0.0
$$

By using Eq. (A.3.3a) and (A.3.3b) Eq. (A.3.17) can be reduced to

$$
\frac{\partial \widetilde{\rho}_{m}}{\partial t}+\widetilde{u}_{m, i} \frac{\partial \tilde{\rho}_{m}}{\partial x_{i}}=0.0
$$

Finally, considering the mixture continuity equation given by

$$
\frac{\partial \widetilde{\rho}_{m}}{\partial t}+\frac{\partial \widetilde{\rho}_{m} \widetilde{u}_{m, i}}{\partial x_{i}}=0.0
$$

and using the chain rule in conjunction with Eq. (A.3.18) gives

$$
\frac{\partial \tilde{u}_{m, i}}{\partial x_{i}}=0.0
$$

which gives the time averaged form or this relation as

$$
\frac{\partial u_{m, i}}{\partial x_{i}}=0.0
$$

which completes the proof. 


\section{A.4 Volume Fraction Equation for SFST Model}

Since there are originally two continuity equations for two phase flow, another equation in addition to the mixture continuity equation must be used to determine the volume fraction of one of the phases. Consider just the single phase continuity equation for phase $\alpha$ as

$$
\frac{\partial\left(\tilde{\mathrm{r}}_{\alpha} \rho_{\alpha}\right)}{\partial \mathrm{t}}+\frac{\partial}{\partial \mathrm{x}_{\mathrm{i}}}\left(\tilde{\mathrm{r}}_{\alpha} \rho_{\alpha} \tilde{\mathrm{u}}_{\alpha, \mathrm{i}}\right)=0
$$

Time averaging Eq. (A.4.1) gives

$$
\frac{\partial\left(\mathrm{r}_{\alpha} \rho_{\alpha}\right)}{\partial \mathrm{t}}+\frac{\partial}{\partial \mathrm{x}_{\mathrm{i}}}\left(\mathrm{r}_{\alpha} \rho_{\alpha} \mathrm{u}_{\alpha, \mathrm{i}}\right)=-\frac{\partial}{\partial \mathrm{x}_{\mathrm{i}}}\left(\rho_{\alpha} \overline{\mathrm{r}_{\alpha}^{\prime} \mathrm{u}_{\alpha, \mathrm{i}}^{\prime}}\right)
$$

The goal is to write Eq. (A.4.2) in terms of the mixture velocity. It can be shown that

$$
r_{\alpha} \rho_{\alpha} u_{\alpha}=r_{\alpha} \rho_{\alpha} u_{m}+\frac{r_{\alpha} \rho_{\alpha} r_{\beta} \rho_{\beta}}{\rho_{m}}\left(u_{\alpha}-u_{\beta}\right)
$$

which is substituted into Eq. (A.4.2) to give

$$
\frac{\partial\left(r_{\alpha} \rho_{\alpha}\right)}{\partial t}+\frac{\partial}{\partial x_{i}}\left(r_{\alpha} \rho_{\alpha} u_{m, i}\right)=-\frac{\partial}{\partial x_{i}}\left[\frac{r_{\alpha} \rho_{\alpha} r_{\beta} \rho_{\beta}}{\rho_{m}}\left(u_{\alpha, i}-u_{\beta, i}\right)\right]-\frac{\partial}{\partial x_{i}}\left(\rho_{\alpha} \overline{r_{\alpha}^{\prime} u_{\alpha, i}^{\prime}}\right)
$$

as the volume fraction equation for the second phase. In the present model the last term in Eq. (A.4.4) is modeled using a gradient diffusion approach where

and

$$
\rho_{\alpha} \overline{r_{\alpha}^{\prime} u_{\alpha, i}^{\prime}}=-\rho_{\alpha} \frac{\Gamma_{\mathrm{T}}}{\rho_{\mathrm{m}}} \frac{\partial \mathrm{r}_{\alpha}}{\partial \mathrm{x}_{\mathrm{i}}}
$$

$$
\Gamma_{\mathrm{T}}=\frac{v_{\mathrm{T}} \rho_{\mathrm{m}}}{\sigma_{\mathrm{r}}}
$$

This gives the modeled form of the volume fraction equation as

$$
\frac{\partial\left(\mathrm{r}_{\alpha} \rho_{\alpha}\right)}{\partial \mathrm{t}}+\frac{\partial}{\partial \mathrm{x}_{\mathrm{i}}}\left(\mathrm{r}_{\alpha} \rho_{\alpha} \mathrm{u}_{\mathrm{m}, \mathrm{i}}\right)=-\frac{\partial}{\partial \mathrm{x}_{\mathrm{i}}}\left[\frac{\mathrm{r}_{\alpha} \rho_{\alpha} \mathrm{r}_{\beta} \rho_{\beta}}{\rho_{\mathrm{m}}}\left(\mathrm{u}_{\alpha, \mathrm{i}}-\mathrm{u}_{\beta, \mathrm{i}}\right)\right]-\frac{\partial}{\partial \mathrm{x}_{\mathrm{i}}}\left[\rho_{\alpha} \frac{\Gamma_{\mathrm{T}}}{\rho_{\mathrm{m}}} \frac{\partial \mathrm{r}_{\alpha}}{\partial \mathrm{x}_{\mathrm{i}}}\right]
$$


This may further be put into a form similar to a generic scalar transport equation by using $\rho_{\mathrm{m}}$ and the fact that the divergence of the mixture velocity is zero as was shown in Appendix A.3. Canceling $\rho_{\alpha}$ and multiplying both sides of Eq. (A.4.7) by $\rho_{\mathrm{m}}$ gives

$$
\begin{gathered}
\frac{\partial\left(\mathrm{r}_{\alpha} \rho_{\mathrm{m}}\right)}{\partial \mathrm{t}}+\frac{\partial}{\partial \mathrm{x}_{\mathrm{i}}}\left(\mathrm{r}_{\alpha} \rho_{\mathrm{m}} \mathrm{u}_{\mathrm{m}, \mathrm{i}}\right)=-\rho_{\mathrm{m}} \frac{\partial}{\partial \mathrm{x}_{\mathrm{i}}}\left[\frac{\Gamma_{\mathrm{T}}}{\rho_{\mathrm{m}}} \frac{\partial \mathrm{r}_{\alpha}}{\partial \mathrm{x}_{\mathrm{i}}}\right] \\
-\rho_{\mathrm{m}} \frac{\partial}{\partial \mathrm{x}_{\mathrm{i}}}\left[\frac{\mathrm{r}_{\alpha} \mathrm{r}_{\beta} \rho_{\beta}}{\rho_{\mathrm{m}}}\left(\mathrm{u}_{\alpha, \mathrm{i}}-\mathrm{u}_{\beta, \mathrm{i}}\right)\right]
\end{gathered}
$$

where $\rho_{\mathrm{m}}$ has been taken inside the terms on the left side using the fact that the divergence of the mixture velocity is zero. Making a slight approximation in the diffusion term, Eq. (A.4.8) can be written in the form of a generic scalar transport equation with one extra term that is a function of the slip velocity as

$$
\frac{\partial\left(\mathrm{r}_{\alpha} \rho_{\mathrm{m}}\right)}{\partial \mathrm{t}}+\frac{\partial}{\partial \mathrm{x}_{\mathrm{i}}}\left(\mathrm{r}_{\alpha} \rho_{\mathrm{m}} \mathrm{u}_{\mathrm{m}, \mathrm{i}}\right)=-\rho_{\mathrm{m}} \frac{\partial}{\partial \mathrm{x}_{\mathrm{i}}}\left[\frac{\mathrm{r}_{\alpha} \mathrm{r}_{\beta} \rho_{\beta}}{\rho_{\mathrm{m}}}\left(\mathrm{u}_{\alpha, \mathrm{i}}-\mathrm{u}_{\beta, \mathrm{i}}\right)\right]-\frac{\partial}{\partial \mathrm{x}_{\mathrm{i}}}\left[\Gamma_{\mathrm{T}} \frac{\partial \mathrm{r}_{\alpha}}{\partial \mathrm{x}_{\mathrm{i}}}\right]
$$

which gives the final form of the modeled volume fraction equation for the SFST models. 


\section{A.5 Mixture Momentum Equation for SFST Models}

The starting point for this derivation is the individual phase momentum equations as given by Ishii. Given two phases, the individual phase momentum equations are given by

$$
\begin{gathered}
\frac{\partial\left(\mathrm{r}_{\alpha} \rho_{\alpha} \mathrm{u}_{\alpha, \mathrm{i}}\right)}{\partial \mathrm{t}}+\frac{\partial}{\partial \mathrm{x}_{\mathrm{j}}}\left(\mathrm{r}_{\alpha} \rho_{\alpha} \mathrm{u}_{\alpha, \mathrm{i}} \mathrm{u}_{\alpha, \mathrm{j}}\right)=-\frac{\partial \mathrm{r}_{\alpha} \mathrm{p}_{\alpha}}{\partial \mathrm{x}_{\mathrm{i}}}+\frac{\partial}{\partial \mathrm{x}_{\mathrm{j}}}\left[\mathrm{r}_{\alpha} \tau_{\alpha, \mathrm{i}, \mathrm{j}}\right] \\
+\frac{\partial}{\partial \mathrm{x}_{\mathrm{j}}}\left[\mathrm{r}_{\alpha} \mu_{\alpha}\left(\frac{\partial \mathrm{u}_{\alpha, \mathrm{i}}}{\partial \mathrm{x}_{\mathrm{j}}}+\frac{\partial \mathrm{u}_{\alpha, \mathrm{j}}}{\partial \mathrm{x}_{\mathrm{i}}}\right)\right]+\mathrm{r}_{\alpha} \rho_{\alpha} \mathrm{g}_{\mathrm{i}}+\mathrm{M}_{\alpha \beta}
\end{gathered}
$$

and

$$
\begin{gathered}
\frac{\partial\left(\mathrm{r}_{\beta} \rho_{\beta} \mathrm{u}_{\beta, \mathrm{i}}\right)}{\partial \mathrm{t}}+\frac{\partial}{\partial \mathrm{x}_{\mathrm{j}}}\left(\mathrm{r}_{\beta} \rho_{\beta} \mathrm{u}_{\beta, \mathrm{i}} \mathrm{u}_{\beta, \mathrm{j}}\right)=-\frac{\partial \mathrm{r}_{\beta} \mathrm{p}_{\beta}}{\partial \mathrm{x}_{\mathrm{i}}}+\frac{\partial}{\partial \mathrm{x}_{\mathrm{j}}}\left[\mathrm{r}_{\beta} \tau_{\beta, \mathrm{i}, \mathrm{j}}\right] \\
+\frac{\partial}{\partial \mathrm{x}_{\mathrm{j}}}\left[\mathrm{r}_{\beta} \mu_{\beta}\left(\frac{\partial \mathrm{u}_{\beta, \mathrm{i}}}{\partial \mathrm{x}_{\mathrm{j}}}+\frac{\partial \mathrm{u}_{\beta, \mathrm{j}}}{\partial \mathrm{x}_{\mathrm{i}}}\right)\right]+\mathrm{r}_{\beta} \rho_{\beta} \mathrm{g}_{\mathrm{i}}+\mathrm{M}_{\beta \alpha}
\end{gathered}
$$

Here the time averaged variables have been used and $\tau_{\alpha}$ and $\tau_{\beta}$ represent the time averaged turbulent stresses. Performing Reynolds averaging on the instantaneous equations results in numerous fluctuating correlations, complicating the equations considerably. Writing the time averaged turbulent stresses in the present form is a mathematical simplification for this model that assumes some prescription can be made for the time averaged turbulent stresses. $\mathrm{M}_{\alpha \beta}$ represents any surface force on phase $\alpha$ from phase $\beta . M_{\alpha \beta}$ is essentially a term for any force not already accounted for that arises at the phase interface. Given that $\mathrm{M}_{\alpha \beta}=-\mathrm{M}_{\beta \alpha}$ by Newton's third law, we add Eqs. (A.5.1) and Eq. (A.5.2) to give

$$
\begin{gathered}
\frac{\partial}{\partial t}\left[\frac{\rho_{\mathrm{m}}}{\rho_{\mathrm{m}}}\left(\mathrm{r}_{\alpha} \rho_{\alpha} \mathrm{u}_{\alpha, \mathrm{i}}+\mathrm{r}_{\beta} \rho_{\beta} \mathrm{u}_{\beta, \mathrm{i}}\right)\right]+\frac{\partial}{\partial \mathrm{x}_{\mathrm{j}}}\left(\mathrm{r}_{\alpha} \rho_{\alpha} \mathrm{u}_{\alpha, \mathrm{i}} \mathrm{u}_{\alpha, \mathrm{j}}+\mathrm{r}_{\beta} \rho_{\beta} \mathrm{u}_{\beta, \mathrm{i}} \mathrm{u}_{\beta, \mathrm{j}}\right)= \\
-\frac{\partial}{\partial \mathrm{x}_{\mathrm{i}}}\left(\mathrm{r}_{\alpha} \mathrm{p}_{\alpha}+\mathrm{r}_{\beta} \mathrm{p}_{\beta}\right)+\frac{\partial}{\partial \mathrm{x}_{\mathrm{j}}}\left[\mathrm{r}_{\alpha} \mu_{\alpha}\left(\frac{\partial \mathrm{u}_{\alpha, \mathrm{i}}}{\partial \mathrm{x}_{\mathrm{j}}}+\frac{\partial \mathrm{u}_{\alpha, \mathrm{j}}}{\partial \mathrm{x}_{\mathrm{i}}}\right)+\mathrm{r}_{\beta} \mu_{\beta}\left(\frac{\partial \mathrm{u}_{\beta, \mathrm{i}}}{\partial \mathrm{x}_{\mathrm{j}}}+\frac{\partial \mathrm{u}_{\beta, \mathrm{j}}}{\partial \mathrm{x}_{\mathrm{i}}}\right)\right] \\
+\frac{\partial}{\partial \mathrm{x}_{\mathrm{j}}}\left[\mathrm{r}_{\alpha} \tau_{\alpha, \mathrm{i}, \mathrm{j}}+\mathrm{r}_{\beta} \tau_{\beta, \mathrm{i}, \mathrm{j}}\right]+\left(\mathrm{r}_{\alpha} \rho_{\alpha}+\mathrm{r}_{\beta} \rho_{\beta}\right) \mathrm{g}_{\mathrm{i}}
\end{gathered}
$$


Realizing that under the model assumptions $\tau_{\alpha}=\tau_{\beta}$ and $p_{\alpha}=p_{\beta}$ and using the definitions for $\rho_{\mathrm{m}}$ and $\mathrm{u}_{\mathrm{m}}$ gives

$$
\begin{gathered}
\frac{\partial}{\partial t}\left[\rho_{\mathrm{m}} \mathrm{u}_{\mathrm{m}, \mathrm{i}}\right]+\frac{\partial}{\partial \mathrm{x}_{\mathrm{j}}}\left(\mathrm{r}_{\alpha} \rho_{\alpha} \mathrm{u}_{\alpha, \mathrm{i}} \mathrm{u}_{\alpha, \mathrm{j}}+\mathrm{r}_{\beta} \rho_{\beta} \mathrm{u}_{\beta, \mathrm{i}} \mathrm{u}_{\beta, \mathrm{j}}\right)=-\frac{\partial \mathrm{p}}{\partial \mathrm{x}_{\mathrm{i}}} \\
+\frac{\partial}{\partial \mathrm{x}_{\mathrm{j}}}\left[\mathrm{r}_{\alpha} \mu_{\alpha}\left(\frac{\partial \mathrm{u}_{\alpha, \mathrm{i}}}{\partial \mathrm{x}_{\mathrm{j}}}+\frac{\partial \mathrm{u}_{\alpha, \mathrm{j}}}{\partial \mathrm{x}_{\mathrm{i}}}\right)+\mathrm{r}_{\beta} \mu_{\beta}\left(\frac{\partial \mathrm{u}_{\beta, \mathrm{i}}}{\partial \mathrm{x}_{\mathrm{j}}}+\frac{\partial \mathrm{u}_{\beta, \mathrm{j}}}{\partial \mathrm{x}_{\mathrm{i}}}\right)\right]+\frac{\partial \tau_{\mathrm{i}, \mathrm{j}}}{\partial \mathrm{x}_{\mathrm{j}}}+\rho_{\mathrm{m}} \mathrm{g}_{\mathrm{i}}
\end{gathered}
$$

Consider the convective and viscous stress terms individually. The convective term is written as

$$
\begin{aligned}
& \left(r_{\alpha} \rho_{\alpha} u_{\alpha, i} u_{\alpha, j}+r_{\beta} \rho_{\beta} u_{\beta, i} u_{\beta, j}\right)=\frac{\rho_{m}}{\rho_{m}}\left(r_{\alpha} \rho_{\alpha} u_{\alpha, i} u_{\alpha, j}+r_{\beta} \rho_{\beta} u_{\beta, i} u_{\beta, j}\right)+ \\
& {\left[\frac{1}{\rho_{m}} r_{\alpha} \rho_{\alpha} r_{\beta} \rho_{\beta}\left(u_{\alpha, i}-u_{\beta, i}\right)\left(u_{\alpha, j}-u_{\beta, j}\right)\right]-\left[\frac{1}{\rho_{m}} r_{\alpha} \rho_{\alpha} r_{\beta} \rho_{\beta}\left(u_{\alpha, i}-u_{\beta, i}\right)\left(u_{\alpha, j}-u_{\beta, j}\right)\right]} \\
& =\frac{1}{\rho_{m}}\left\{\left(r_{\alpha} \rho_{\alpha}+r_{\beta} \rho_{\beta}\right) r_{\alpha} \rho_{\alpha} u_{\alpha, i} u_{\alpha, j}+\left(r_{\alpha} \rho_{\alpha}+r_{\beta} \rho_{\beta}\right) r_{\beta} \rho_{\beta} u_{\beta, i} u_{\beta, j}\right. \\
& \left.-r_{\beta} \rho_{\beta} r_{\alpha} \rho_{\alpha} u_{\alpha, i} u_{\alpha, j}-r_{\beta} \rho_{\beta} r_{\alpha} \rho_{\alpha} u_{\beta, i} u_{\beta, j}+r_{\beta} \rho_{\beta} r_{\alpha} \rho_{\alpha} u_{\beta, i} u_{\alpha, j}-r_{\beta} \rho_{\beta} r_{\alpha} \rho_{\alpha} u_{\alpha, i} u_{\beta, j}\right\} \\
& +\left[\frac{1}{\rho_{m}} r_{\alpha} \rho_{\alpha} r_{\beta} \rho_{\beta}\left(u_{\alpha, i}-u_{\beta, i}\right)\left(u_{\alpha, j}-u_{\beta, j}\right)\right] \\
& =\frac{1}{\rho_{m}}\left[\rho_{m} \rho_{m} \frac{\left(r_{\alpha} \rho_{\alpha} u_{\alpha, i}+r_{\beta} \rho_{\beta} u_{\beta, i}\right)}{\rho_{m}} \frac{\left(r_{\alpha} \rho_{\alpha} u_{\alpha, j}+r_{\beta} \rho_{\beta} u_{\beta, j}\right)}{\rho_{m}}\right]+\left[\frac{1}{\rho_{m}} r_{\alpha} \rho_{\alpha} r_{\beta} \rho_{\beta}\left(u_{\alpha, i}-u_{\beta, i}\right)\left(u_{\alpha, j}-u_{\beta, j}\right)\right] \\
& =\left[\rho_{m} u_{m, i} u_{m, j}\right]+\left[\frac{r_{\alpha} \rho_{\alpha} r_{\beta} \rho_{\beta}}{\rho_{m}}\left(u_{\alpha, i}-u_{\beta, i}\right)\left(u_{\alpha, j}-u_{\beta, j}\right)\right]
\end{aligned}
$$


The viscous term may be written as

$$
\begin{aligned}
& {\left[\mathrm{r}_{\alpha} \mu_{\alpha}\left(\frac{\partial \mathrm{u}_{\alpha, \mathrm{i}}}{\partial \mathrm{x}_{\mathrm{j}}}+\frac{\partial \mathrm{u}_{\alpha, \mathrm{j}}}{\partial \mathrm{x}_{\mathrm{i}}}\right)+\mathrm{r}_{\beta} \mu_{\beta}\left(\frac{\partial \mathrm{u}_{\beta, \mathrm{i}}}{\partial \mathrm{x}_{\mathrm{j}}}+\frac{\partial \mathrm{u}_{\beta, \mathrm{j}}}{\partial \mathrm{x}_{\mathrm{i}}}\right)\right]=} \\
& {\left[r_{\alpha} \mu_{\alpha} \frac{\partial}{\partial x_{j}}\left(\frac{\left(r_{\alpha} \rho_{\alpha} u_{\alpha, i}+r_{\beta} \rho_{\beta} u_{\alpha, i}\right)}{\rho_{m}}\right)+r_{\beta} \mu_{\beta} \frac{\partial}{\partial x_{j}}\left(\frac{\left(r_{\alpha} \rho_{\alpha} u_{\beta, i}+r_{\beta} \rho_{\beta} u_{\beta, i}\right)}{\rho_{m}}\right)\right]+} \\
& {\left[r_{\alpha} \mu_{\alpha} \frac{\partial}{\partial x_{i}}\left(\frac{\left(r_{\alpha} \rho_{\alpha} u_{\alpha, j}+r_{\beta} \rho_{\beta} u_{\alpha, j}\right)}{\rho_{m}}\right)+r_{\beta} \mu_{\beta} \frac{\partial}{\partial x_{i}}\left(\frac{\left(r_{\alpha} \rho_{\alpha} u_{\beta, j}+r_{\beta} \rho_{\beta} u_{\beta, j}\right)}{\rho_{m}}\right)\right]=} \\
& r_{\alpha} \mu_{\alpha} \frac{\partial}{\partial x_{j}}\left(\frac{\left(r_{\alpha} \rho_{\alpha} u_{\alpha, i}+r_{\beta} \rho_{\beta} u_{\beta, i}\right)}{\rho_{m}}+\frac{\left(r_{\beta} \rho_{\beta} u_{\alpha, i}-r_{\beta} \rho_{\beta} u_{\beta, i}\right)}{\rho_{m}}\right) \\
& +r_{\beta} \mu_{\beta} \frac{\partial}{\partial x_{j}}\left(\frac{\left(r_{\alpha} \rho_{\alpha} u_{\alpha, i}+r_{\beta} \rho_{\beta} u_{\beta, i}\right)}{\rho_{m}}+\frac{\left(r_{\alpha} \rho_{\alpha} u_{\beta, i}-r_{\alpha} \rho_{\alpha} u_{\alpha, i}\right)}{\rho_{m}}\right) \\
& r_{\alpha} \mu_{\alpha} \frac{\partial}{\partial x_{i}}\left(\frac{\left(r_{\alpha} \rho_{\alpha} u_{\alpha, j}+r_{\beta} \rho_{\beta} u_{\beta, j}\right)}{\rho_{m}}+\frac{\left(r_{\beta} \rho_{\beta} u_{\alpha, j}-r_{\beta} \rho_{\beta} u_{\beta, j}\right)}{\rho_{m}}\right) \\
& +r_{\beta} \mu_{\beta} \frac{\partial}{\partial x_{i}}\left(\frac{\left(r_{\alpha} \rho_{\alpha} u_{\alpha, j}+r_{\beta} \rho_{\beta} u_{\beta, j}\right)}{\rho_{m}}+\frac{\left(r_{\alpha} \rho_{\alpha} u_{\beta, j}-r_{\alpha} \rho_{\alpha} u_{\alpha, j}\right)}{\rho_{m}}\right)= \\
& \left(\mathrm{r}_{\alpha} \mu_{\alpha}+\mathrm{r}_{\beta} \mu_{\beta}\right) \frac{\partial \mathrm{u}_{\mathrm{m}, \mathrm{i}}}{\partial \mathrm{x}_{\mathrm{j}}}+\left(\mathrm{r}_{\alpha} \mu_{\alpha}+\mathrm{r}_{\beta} \mu_{\beta}\right) \frac{\partial \mathrm{u}_{\mathrm{m}, \mathrm{j}}}{\partial \mathrm{x}_{\mathrm{i}}} \\
& +\mathrm{r}_{\alpha} \mu_{\alpha} \frac{\partial}{\partial \mathrm{x}_{\mathrm{j}}}\left(\frac{\mathrm{r}_{\beta} \rho_{\beta}}{\rho_{\mathrm{m}}}\left(\mathrm{u}_{\alpha, \mathrm{i}}-\mathrm{u}_{\beta, \mathrm{i}}\right)\right)-\mathrm{r}_{\beta} \mu_{\beta} \frac{\partial}{\partial \mathrm{x}_{\mathrm{j}}}\left(\frac{\mathrm{r}_{\alpha} \rho_{\alpha}}{\rho_{\mathrm{m}}}\left(\mathrm{u}_{\alpha, \mathrm{i}}-\mathrm{u}_{\beta, \mathrm{i}}\right)\right) \\
& +r_{\alpha} \mu_{\alpha} \frac{\partial}{\partial x_{i}}\left(\frac{r_{\beta} \rho_{\beta}}{\rho_{m}}\left(u_{\alpha, j}-u_{\beta, j}\right)\right)-r_{\beta} \mu_{\beta} \frac{\partial}{\partial x_{i}}\left(\frac{r_{\alpha} \rho_{\alpha}}{\rho_{m}}\left(u_{\alpha, j}-u_{\beta, j}\right)\right)= \\
& \mu_{\mathrm{m}}\left(\frac{\partial \mathrm{u}_{\mathrm{m}, \mathrm{i}}}{\partial \mathrm{x}_{\mathrm{j}}}+\frac{\partial \mathrm{u}_{\mathrm{m}, \mathrm{j}}}{\partial \mathrm{x}_{\mathrm{i}}}\right)+\mathrm{r}_{\alpha} \mu_{\alpha} \frac{\partial}{\partial \mathrm{x}_{\mathrm{j}}}\left(\frac{\mathrm{r}_{\beta} \rho_{\beta}}{\rho_{\mathrm{m}}}\left(\mathrm{u}_{\alpha, \mathrm{i}}-\mathrm{u}_{\beta, \mathrm{i}}\right)\right)-\mathrm{r}_{\beta} \mu_{\beta} \frac{\partial}{\partial \mathrm{x}_{\mathrm{j}}}\left(\frac{\mathrm{r}_{\alpha} \rho_{\alpha}}{\rho_{\mathrm{m}}}\left(\mathrm{u}_{\alpha, \mathrm{i}}-\mathrm{u}_{\beta, \mathrm{i}}\right)\right) \\
& +r_{\alpha} \mu_{\alpha} \frac{\partial}{\partial x_{i}}\left(\frac{r_{\beta} \rho_{\beta}}{\rho_{m}}\left(u_{\alpha, j}-u_{\beta, j}\right)\right)-r_{\beta} \mu_{\beta} \frac{\partial}{\partial x_{i}}\left(\frac{r_{\alpha} \rho_{\alpha}}{\rho_{m}}\left(u_{\alpha, j}-u_{\beta, j}\right)\right)
\end{aligned}
$$


Collecting the viscous and convective terms gives

$$
\begin{gathered}
\frac{\partial}{\partial t}\left[\rho_{\mathrm{m}} \mathrm{u}_{\mathrm{m}, \mathrm{i}}\right]+\frac{\partial}{\partial \mathrm{x}_{\mathrm{j}}}\left[\rho_{\mathrm{m}} \mathrm{u}_{\mathrm{m}, \mathrm{i}} \mathrm{u}_{\mathrm{m}, \mathrm{j}}\right]=-\frac{\partial \mathrm{p}}{\partial \mathrm{x}_{\mathrm{i}}}-\frac{\partial}{\partial \mathrm{x}_{\mathrm{j}}}\left[\frac{\mathrm{r}_{\alpha} \rho_{\alpha} \mathrm{r}_{\beta} \rho_{\beta}}{\rho_{\mathrm{m}}}\left(\mathrm{u}_{\alpha, \mathrm{i}}-\mathrm{u}_{\beta, \mathrm{i}}\right)\left(\mathrm{u}_{\alpha, \mathrm{j}}-\mathrm{u}_{\beta, \mathrm{j}}\right)\right] \\
+\frac{\partial}{\partial \mathrm{x}_{\mathrm{j}}}\left[\mathrm{r}_{\alpha} \mu_{\alpha} \frac{\partial}{\partial \mathrm{x}_{\mathrm{j}}}\left(\frac{\mathrm{r}_{\beta} \rho_{\beta}}{\rho_{\mathrm{m}}}\left(\mathrm{u}_{\alpha, \mathrm{i}}-\mathrm{u}_{\beta, \mathrm{i}}\right)\right)-\mathrm{r}_{\beta} \mu_{\beta} \frac{\partial}{\partial \mathrm{x}_{\mathrm{j}}}\left(\frac{\mathrm{r}_{\alpha} \rho_{\alpha}}{\rho_{\mathrm{m}}}\left(\mathrm{u}_{\alpha, \mathrm{i}}-\mathrm{u}_{\beta, \mathrm{i}}\right)\right)\right] \\
+\frac{\partial}{\partial \mathrm{x}_{\mathrm{j}}}\left[\mathrm{r}_{\alpha} \mu_{\alpha} \frac{\partial}{\partial \mathrm{x}_{\mathrm{i}}}\left(\frac{\mathrm{r}_{\beta} \rho_{\beta}}{\rho_{\mathrm{m}}}\left(\mathrm{u}_{\alpha, \mathrm{j}}-\mathrm{u}_{\beta, \mathrm{j}}\right)\right)-\mathrm{r}_{\beta} \mu_{\beta} \frac{\partial}{\partial \mathrm{x}_{\mathrm{i}}}\left(\frac{\mathrm{r}_{\alpha} \rho_{\alpha}}{\rho_{\mathrm{m}}}\left(\mathrm{u}_{\alpha, \mathrm{j}}-\mathrm{u}_{\beta, \mathrm{j}}\right)\right)\right] \\
+\frac{\partial}{\partial \mathrm{x}_{\mathrm{j}}}\left(\mu_{\mathrm{m}}\left(\frac{\partial \mathrm{u}_{\mathrm{m}, \mathrm{i}}}{\partial \mathrm{x}_{\mathrm{j}}}+\frac{\partial \mathrm{u}_{\mathrm{m}, \mathrm{j}}}{\partial \mathrm{x}_{\mathrm{i}}}\right)\right)+\frac{\partial \tau_{\mathrm{i}, \mathrm{j}}}{\partial \mathrm{x}_{\mathrm{j}}}+\rho_{\mathrm{m}} \mathrm{g}_{\mathrm{i}}
\end{gathered}
$$

which can be written in a more compact form by using $\mathrm{u}_{\mathrm{S}, \mathrm{i}}=\left(\mathrm{u}_{\alpha, \mathrm{i}}-\mathrm{u}_{\beta, \mathrm{i}}\right)$ as

$$
\begin{aligned}
\frac{\partial}{\partial t}\left[\rho_{\mathrm{m}} \mathrm{u}_{\mathrm{m}, \mathrm{i}}\right]+\frac{\partial}{\partial \mathrm{x}_{\mathrm{j}}}\left[\rho_{\mathrm{m}} \mathrm{u}_{\mathrm{m}, \mathrm{i}} \mathrm{u}_{\mathrm{m}, \mathrm{j}}\right]=-\frac{\partial \mathrm{p}}{\partial \mathrm{x}_{\mathrm{i}}}-\frac{\partial}{\partial \mathrm{x}_{\mathrm{j}}}\left[\frac{\mathrm{r}_{\alpha} \rho_{\alpha} \mathrm{r}_{\beta} \rho_{\beta}}{\rho_{\mathrm{m}}}\left(\mathrm{u}_{\mathrm{s}, \mathrm{i}} \mathrm{u}_{\mathrm{s}, \mathrm{j}}\right)\right] \\
+\frac{\partial}{\partial \mathrm{x}_{\mathrm{j}}}\left[\mathrm{r}_{\alpha} \mu_{\alpha} \frac{\partial}{\partial \mathrm{x}_{\mathrm{j}}}\left(\frac{\mathrm{r}_{\beta} \rho_{\beta}}{\rho_{\mathrm{m}}} \mathrm{u}_{\mathrm{S}, \mathrm{i}}\right)-\mathrm{r}_{\beta} \mu_{\beta} \frac{\partial}{\partial \mathrm{x}_{\mathrm{j}}}\left(\frac{\mathrm{r}_{\alpha} \rho_{\alpha}}{\rho_{\mathrm{m}}} \mathrm{u}_{\mathrm{s}, \mathrm{i}}\right)\right] \\
+\frac{\partial}{\partial \mathrm{x}_{\mathrm{j}}}\left[\mathrm{r}_{\alpha} \mu_{\alpha} \frac{\partial}{\partial \mathrm{x}_{\mathrm{i}}}\left(\frac{\mathrm{r}_{\beta} \rho_{\beta}}{\rho_{\mathrm{m}}} \mathrm{u}_{\mathrm{s}, \mathrm{j}}\right)-\mathrm{r}_{\beta} \mu_{\beta} \frac{\partial}{\partial \mathrm{x}_{\mathrm{i}}}\left(\frac{\mathrm{r}_{\alpha} \rho_{\alpha}}{\rho_{\mathrm{m}}} \mathrm{u}_{\mathrm{s}, \mathrm{j}}\right)\right] \\
+\frac{\partial}{\partial \mathrm{x}_{\mathrm{j}}}\left(\mu_{\mathrm{m}}\left(\frac{\partial \mathrm{u}_{\mathrm{m}, \mathrm{i}}}{\partial \mathrm{x}_{\mathrm{j}}}+\frac{\partial \mathrm{u}_{\mathrm{m}, \mathrm{j}}}{\partial \mathrm{x}_{\mathrm{i}}}\right)\right)+\frac{\partial \tau_{\mathrm{i}, \mathrm{j}}}{\partial \mathrm{x}_{\mathrm{j}}}+\rho_{\mathrm{m}} \mathrm{g}_{\mathrm{i}}
\end{aligned}
$$

Eq. (A.5.8) represents the mixture momentum equation with no assumptions for the turbulent stresses. $\tau_{\mathrm{ij}}$ can be modeled in several ways; following Ishii it is taken to be given by a Boussinesq eddy viscosity model using the mixture velocity where

$$
\tau_{\mathrm{ij}}=\mu_{\mathrm{T}}\left(\frac{\partial \mathrm{u}_{\mathrm{m}, \mathrm{i}}}{\partial \mathrm{x}_{\mathrm{j}}}+\frac{\partial \mathrm{u}_{\mathrm{m}, \mathrm{j}}}{\partial \mathrm{x}_{\mathrm{i}}}\right)-\frac{2}{3} \rho_{\mathrm{m}} k \delta_{\mathrm{ij}}
$$


which gives the modeled form of the mixture momentum equations as

$$
\begin{aligned}
\frac{\partial}{\partial t}\left[\rho_{\mathrm{m}} \mathrm{u}_{\mathrm{m}, \mathrm{i}}\right]+\frac{\partial}{\partial \mathrm{x}_{\mathrm{j}}}\left[\rho_{\mathrm{m}} \mathrm{u}_{\mathrm{m}, \mathrm{i}} \mathrm{u}_{\mathrm{m}, \mathrm{j}}\right]=-\frac{\partial \mathrm{P}}{\partial \mathrm{x}_{\mathrm{i}}}-\frac{\partial}{\partial \mathrm{x}_{\mathrm{j}}}\left[\frac{\mathrm{r}_{\alpha} \rho_{\alpha} \mathrm{r}_{\beta} \rho_{\beta}}{\rho_{\mathrm{m}}}\left(\mathrm{u}_{\mathrm{s}, \mathrm{i}} \mathrm{u}_{\mathrm{S}, \mathrm{j}}\right)\right] \\
+\frac{\partial}{\partial \mathrm{x}_{\mathrm{j}}}\left[\mathrm{r}_{\alpha} \mu_{\alpha} \frac{\partial}{\partial \mathrm{x}_{\mathrm{j}}}\left(\frac{\mathrm{r}_{\beta} \rho_{\beta}}{\rho_{\mathrm{m}}} \mathrm{u}_{\mathrm{s}, \mathrm{i}}\right)-\mathrm{r}_{\beta} \mu_{\beta} \frac{\partial}{\partial \mathrm{x}_{\mathrm{j}}}\left(\frac{\mathrm{r}_{\alpha} \rho_{\alpha}}{\rho_{\mathrm{m}}} \mathrm{u}_{\mathrm{S}, \mathrm{i}}\right)\right] \\
+\frac{\partial}{\partial \mathrm{x}_{\mathrm{j}}}\left[\mathrm{r}_{\alpha} \mu_{\alpha} \frac{\partial}{\partial \mathrm{x}_{\mathrm{i}}}\left(\frac{\mathrm{r}_{\beta} \rho_{\beta}}{\rho_{\mathrm{m}}} \mathrm{u}_{\mathrm{s}, \mathrm{j}}\right)-\mathrm{r}_{\beta} \mu_{\beta} \frac{\partial}{\partial \mathrm{x}_{\mathrm{i}}}\left(\frac{\mathrm{r}_{\alpha} \rho_{\alpha}}{\rho_{\mathrm{m}}} \mathrm{u}_{\mathrm{S}, \mathrm{j}}\right)\right] \\
+\frac{\partial}{\partial \mathrm{x}_{\mathrm{j}}}\left(\left(\mu_{\mathrm{m}}+\mu_{\mathrm{T}}\right)\left(\frac{\partial \mathrm{u}_{\mathrm{m}, \mathrm{i}}}{\partial \mathrm{x}_{\mathrm{j}}}+\frac{\partial \mathrm{u}_{\mathrm{m}, \mathrm{j}}}{\partial \mathrm{x}_{\mathrm{i}}}\right)\right)+\rho_{\mathrm{m}} \mathrm{g}_{\mathrm{i}}
\end{aligned}
$$

where $\mathrm{P}$ is a modified pressure that is given by

$$
\mathrm{P}=\mathrm{p}+\frac{2}{3} \rho_{\mathrm{m}} k
$$

Four additional terms involving the molecular viscosity appear in Eq. (A.5.10) on the second and third lines and represent the additional molecular stresses that result because of the slip velocity. The influence of these additional viscous terms is typically found to be negligible for a wide range of flows. For instance in convection dominated flows where the mixture velocity is much greater than the slip velocity, the relative contribution to stresses of these additional viscous terms will be small. Also, in a turbulent flow the eddy viscosity is typically significantly greater in magnitude that the molecular viscosity. While traditionally it seems that these terms have been neglected altogether, they are included in the present derivation for the sake of completeness. However, these terms were neglected in all of the applications involving the SFST model and the actual momentum equation that was typically solved is given by

$$
\begin{gathered}
\frac{\partial}{\partial t}\left[\rho_{m} u_{m, i}\right]+\frac{\partial}{\partial x_{j}}\left[\rho_{m} u_{m, i} u_{m, j}\right]=-\frac{\partial}{\partial x_{j}}\left[\frac{r_{\alpha} \rho_{\alpha} r_{\beta} \rho_{\beta}}{\rho_{m}}\left(u_{s, i} u_{S, j}\right)\right] \\
-\frac{\partial P}{\partial x_{i}}+\frac{\partial}{\partial x_{j}}\left(\left(\mu_{m}+\mu_{T}\right)\left(\frac{\partial u_{m, i}}{\partial x_{j}}+\frac{\partial u_{m, j}}{\partial x_{i}}\right)\right)+\rho_{m} g_{i}
\end{gathered}
$$




\section{A.6 Derivation of Terminal Velocity Relationships}

\section{Transient form of Terminal Velocity in Stokes Regime}

Assumptions:

1. Droplet is formed and initially moves with the speed of the surrounding fluid.

2. Coefficient of drag is given by $C_{D}=24 / R_{P}$.

3. $\beta$ denotes continuous phase, $\alpha$ denotes disperse phase.

Basic Relations and Definitions:

Volume of droplet: $\quad \mathrm{V}=\frac{\pi \mathrm{d}_{\mathrm{p}}{ }^{3}}{6} \quad$ Frontal area of droplet: $\quad \mathrm{A}=\frac{\pi \mathrm{d}_{\mathrm{p}}{ }^{2}}{4}$

Mass of droplet: $\quad M_{d p}=\rho_{\alpha} V \quad$ Time constant: $\quad \tau=\frac{4 \rho_{\alpha} d_{p}{ }^{2}}{72 \mu_{\beta}}$

Drop Reynolds number: $\operatorname{Re}_{p}=\frac{\rho_{\beta} u_{s} d_{p}}{\mu_{\beta}} \quad$ Buoyancy: $\quad b=\frac{g\left(\rho_{\beta}-\rho_{\alpha}\right)}{\rho_{\alpha}}$

Solution:

$$
\begin{gathered}
M_{d p} \frac{d u_{S}}{d t}=F_{D}+F_{G}+F_{B} \\
M_{d p} \frac{d u_{S}}{d t}=-\frac{1}{2} C_{D} A \rho_{\beta} u_{S}^{2}-V \rho_{\alpha} g+V \rho_{\beta} g \\
\frac{d u_{S}}{d t}=-\frac{72 \mu_{\beta}}{4 \rho_{\alpha} d_{p}^{2}} u_{S}+\frac{g\left(\rho_{\beta}-\rho_{\alpha}\right)}{\rho_{\alpha}} \\
\frac{d u_{S}}{d t}=-\frac{1}{\tau} u_{S}+b
\end{gathered}
$$

Using an integrating factor gives

$$
\int \frac{d}{d t}\left(e^{t / \tau} u_{s}\right) d t=b \int e^{t / \tau} d t
$$


which gives

$$
\left(e^{t / \tau} u_{s}\right)=b \tau e^{t / \tau}+C
$$

Setting the slip or terminal velocity to zero at $\mathrm{t}=0.0$ gives the constant and allows the slip velocity to be given by

$$
\mathrm{u}_{\mathrm{S}}=\mathrm{b} \tau\left(1-\mathrm{e}^{-\mathrm{t} / \tau}\right)
$$

or

$$
\mathrm{u}_{\mathrm{S}}=\frac{4 \mathrm{gd}_{\mathrm{p}}^{2}}{72 \mu_{\beta}}\left(\rho_{\beta}-\rho_{\alpha}\right)\left(1-\mathrm{e}^{-\mathrm{t} / \tau}\right)
$$

\section{Steady State form of Terminal Velocity in Stokes Regime}

Allowing the time to go to infinity in Eq. (A.6.8) gives

$$
u_{s}=\frac{4 g d_{p}^{2}}{72 \mu_{\beta}}\left(\rho_{\beta}-\rho_{\alpha}\right)
$$

\section{General Steady State Terminal Velocity Relationship}

$$
\begin{gathered}
0.0=-\frac{1}{2} C_{D} A \rho_{\beta} u_{S}^{2}-V \rho_{\alpha} g+V \rho_{\beta} g \\
\frac{3 \rho_{\beta} C_{D}}{4 d_{p}} u_{S}^{2}=g\left(\rho_{\beta}-\rho_{\alpha}\right) \\
u_{S}=\left[\left(\frac{4 g d_{p}}{3 C_{D}}\right)\left(\frac{\left(\rho_{\beta}-\rho_{\alpha}\right)}{\rho_{\beta}}\right)\right]^{1 / 2}
\end{gathered}
$$




\section{Steady State form of Terminal Velocity in Allen's Regime}

Taking the general form of the terminal velocity relationship as the starting point gives

$$
u_{s}^{2}=\left(\frac{4 g d_{p}}{3 C_{D}}\right)\left(\frac{\left(\rho_{\beta}-\rho_{\alpha}\right)}{\rho_{\beta}}\right)
$$

and taking the drag relationship to be given by

$$
\mathrm{C}_{\mathrm{D}}=\frac{18}{\operatorname{Re}_{\mathrm{P}}^{0.6}}
$$

gives

$$
\begin{aligned}
& u_{s}^{2}=\left(\frac{4 g d_{p}}{3(18)}\right)\left(\frac{\left(\rho_{\beta} u_{s} d_{p}\right)^{0.6}}{\mu_{\beta}^{0.6}}\right)\left(\frac{\left(\rho_{\beta}-\rho_{\alpha}\right)}{\rho_{\beta}}\right) \\
& u_{s}^{(2-0.6)}=\left(\frac{4 g}{54}\right)\left(\frac{d_{p}^{(1+0.6)}}{\mu_{\beta}^{0.6}}\right)\left(\frac{\rho_{\beta}-\rho_{\alpha}}{\rho_{\beta}^{(1-0.6)}}\right) \\
& u_{s}=\left[\left(\frac{4 g d_{p}^{1.6}}{54 \mu_{\beta}^{0.6}}\right)\left(\frac{\rho_{\beta}-\rho_{\alpha}}{\rho_{\beta}^{0.4}}\right)\right]^{5 / 7}
\end{aligned}
$$




\section{APPENDIX B: DOCUMENTATION FOR SFST MODEL}

\section{B.1 Introduction}

The following sections are intended to describe how to use the SFST model in conjunction with CFX 4.1 and to describe how to modify the Fortran implementation of the SFST model in the CFX user subroutines. A brief description of the terms that are coded in the model and their physical significance is given. Some instructions for setting up a geometry file, command file, and Fortran file in CFX to run the SFST model are also given. The aspects related to setting up the CFX files concentrates only on what is important for running the SFST model and does not involve aspects that are covered in the CFX Users Manual. A detailed description of the Fortran in the CFX user subroutines that is used to implement the SFST model is also given. This description concentrates on how the source terms, boundary conditions, and the flux limitation in the volume fraction equation source term are implemented in the Fortran. The appropriate locations in the code for the user to set new functions for the slip velocity and for the user to implement functions for the droplet size are also described.

\section{B.2 Overview}

The SFST model is a single fluid, multiphase mixture model that can be used for flows involving two immiscible fluids. The model solves one set of momentum equations and one scalar equation for the volume fraction of the light phase in CFX 4.1. Some of the terms that appear in the SFST model equations are added to the standard single fluid equations in CFX through user Fortran. The user Fortran also includes an equation to specify the mixture density function. The main source term that is added is a convective flux that originates in the volume fraction equation because of the slip velocity. If the $k-\varepsilon$ turbulence model is being solved the user Fortran can also be used to add source terms accounting for buoyancy production and dissipation of turbulent kinetic energy. The current version of the Fortran code that contains the SFST source terms can be coupled with any CFX model. This requires the user to follow a few simple guidelines in setting up the CFX command file and CFX geometry file. The user also has to set a few parameters in the Fortran file that contains the user subroutines that are used to implement the SFST model. The current form of the user Fortran is written in such a way that future development of the model can be accomplished without having to re-code the entire SFST model. 


\section{B.3 Setting up the CFX Files and User Fortran to Run the SFST Model}

To run the SFST model it is necessary to set up three files: the geometry file, the command file, and the Fortran file. These files are to be set up under some guidelines that are explained in the next sections; otherwise the files should be set up like any single fluid CFX model. Text that is written using the courier new font indicates text or parameters that appear in the one of the CFX files.

\section{B.3.1 Setting up the geometry file}

The geometry file should be set up like any typical CFX geometry file; however, the use of the geometry file with the SFST model does require the user to follow the following guidelines.

1. All local IJK coordinate systems that exist in each block of the multi-block structure should have the $\mathrm{J}$ index pointed in the same direction that the gravity vector is pointed in the global XYZ coordinate system. The easiest way to accomplish this is to set the $\mathrm{J}$ index in all of the blocks to coincide with the global $\mathrm{Y}$ vector in the global coordinate system and then to set the gravity vector to -9.81 along the global $\mathrm{Y}$ vector in the CFX command file. It does not matter if the cells or even the blocks are not orthogonal as long as the $\mathrm{J}$ index in the local coordinate system is pointed in the same direction as the global Y index. In the user Fortran only the vertical component of the area on the top of a cell is used for calculating the flux through the top of a non-orthogonal cell.

2. All active blocks where the source terms are to be set should be labeled as BLOCKNUMBER-1 through BLOCK-NUMBER- $N$ where $N$ is the total number of active blocks. Internal active blocks that are used as child constraints and reside inside larger active blocks should not be included in the names in which the source terms are to be set. This is because the source term will automatically be set in an internal active block that is used as a child constraint if it resides inside a larger active block.

3. Internal solid blocks that are used as child constraints and that reside inside larger active blocks should be named INTSOLID1 through INSOLIDN where $N$ is the number of internal solid blocks that are used as child constraints. Internal solid blocks that are not used as child constraints and do not reside inside any active blocks where the source terms are to be set do not need any special naming convention.

5. The user Fortran where the source terms involving the slip velocity are set can handle five types of boundary patches in CFX. The boundaries that the model is capable of handling are walls, inlets, pressure boundaries, mass flow boundaries, and thin surfaces. These five boundary patches are the most common and should be used in setting up the geometry file. 


\section{B.3.2 Setting up the Command File}

Like the geometry file, the CFX command file should be set up like any typical command file that is being set up for a single fluid simulation. The following section describes the parameters that need to be included in the CFX command file to run the SFST model.

1. Under OPTIONS the following parameters should be included in the command file

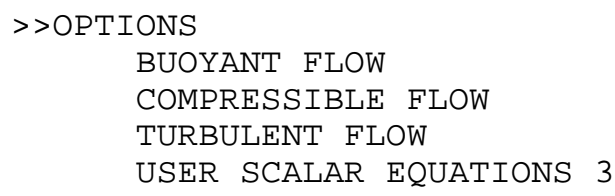

The BUOYANT FLOW option is necessary if the flow acts under the force of gravity and if density differences are important. The COMPRESSIBLE FLOW option allows an equation of state or density equation to be set in the user Fortran subroutine, USRDEN. Later in the command file the flow is specified as WEAKLY COMPRESSIBLE and the derivative of density with respect to pressure is set to zero in the USRDEN subroutine where the mixture density equation is set. The TURBULENT FLOW option is not necessary unless the user desires to run one of the turbulence models and add the source terms to the $k$ and $\varepsilon$ equations. It is necessary for the user to specify three scalar equations. The SFST model only solves one additional scalar equation; however, the model requires storage space for the value of the droplet size and for the cell indicators. The cell indicators are used to implement boundary conditions for the source terms involving the slip velocity as will be described later.

2. Under FORTRAN the following parameters should be included in the command file

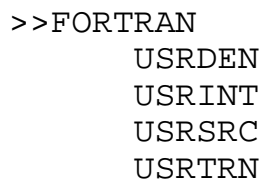

The user may add additional CFX user subroutines if desired. The file SFST.f already has the four subroutines that are listed under FORTRAN; these comprise the main CFX subroutines that are used to set the source terms. The mixture density equation is set in USRDEN, and the source terms are set in USRSRC. USRINT and USRTRN are included since they are popular subroutines that the user may want to use to set initial conditions or to monitor a transient solution. 
3. Under VARIABLE NAMES the following parameters should be included in the command file

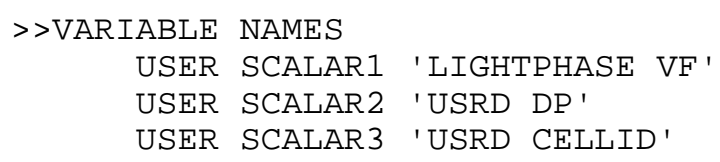

The user subroutine USRSRC uses these names to set the source terms in the volume fraction equation, to store the droplet size, USRD DP, and to store the cell indicator, USRD CELLID. The leading name USRD indicates that the user scalar is not a transport equation but is a storage space that can be used to store information or variables at each cell. Further details on user scalars can be found in the CFX Users Manual.

4. Under PHYSICAL PROPERTIES the following parameters should be included in the command file

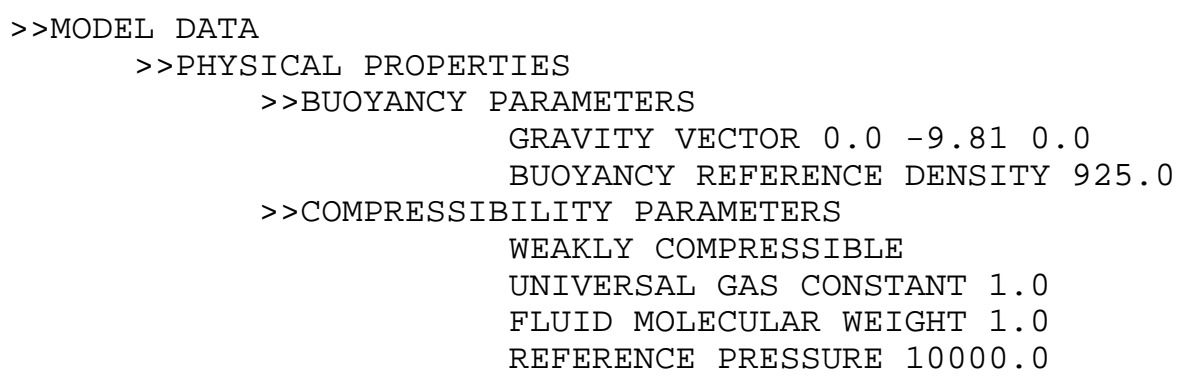

The gravity vector should be set in the same coordinate direction as the local $\mathrm{J}$ index in the active blocks in the geometry file. If the $\mathrm{J}$ index coincides with the global $\mathrm{Y}$ vector in the geometry file, the gravity vector should be set to the same values that are set under GRAVITY VECTOR in the example. The value for BUOYANCY REFERENCE DENSITY is usually set to the average of the unmixed phase densities. The WEAKLY COMPRESSIBLE option under COMPRESSIBILITY PARAMETERS allows a density equation to be specified by the user in USRDEN. Using the WEAKLY COMPRESSIBLE option, CFX will still solve the equations as if they were incompressible with a variable density. The other parameters under COMPRESSIBILITY PARAMETERS are included since the CFX default density equation is the ideal gas law. Setting some reasonable values for these numbers is necessary since they are initially used to initialize the density at the beginning of a simulation. This density is not used in any part of the calculation because it is over written on the first outer iteration by the mixture density equation that is set in user Fortran. 
5. Consideration for which differencing schemes to use is left up to the user for the most part; however, it is recommended in the CFX Users Manual that first order upwinding be used for the volume fraction equation and the density. This is specified in CFX by setting

in the command file that is being used for the SFST model.

6. The initial time should always be started at 0.0 for any simulation except for a restart since the cell indicators are initialized on the first time step or when the time is equal to the time step. This means that for an initial run under TRANSIENT PARAMETERS the user should set

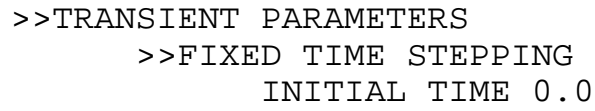

\section{B.3.3 Setting up the Fortran File}

The file SFST.f is a generic file that includes the necessary CFX user subroutines for running the SFST model. It should be copied to the directory where the CFX model is to be constructed. Then the appropriate parameters should be set in the subroutine UBCND, and then any additional Fortran for post processing or setting user boundary conditions should be included. SFST.f is comprised of the subroutines entitled UBCND, UGRDNT, USRINT, USRDEN, USRSRC, USRTRN, and LENGTH. Of these subroutines USRINT, USRDEN, USRSRC, and USRTRN are CFX user subroutines. The other subroutines are called from within the CFX user subroutines.

UBCND is where the main information that is needed by CFX is set by the user. It is the only subroutine in which the user needs to set any parameters to run the SFST model. The parameters, as they appear in UBCND, are listed below along with some additional explanation for setting the parameters.

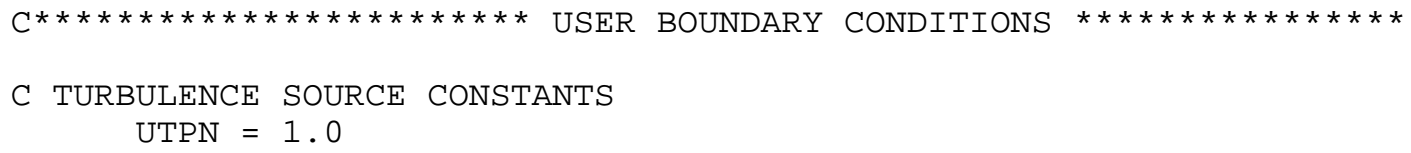

The parameter UTPN is a turbulent prandtl number that appears in the source term that is set in the $k$ equation. The source that is implemented in the SFST model is given by

$$
G_{k}=-\left|g_{i}\right| \overline{u_{i}{ }^{\prime} \rho^{\prime}}=\left|g_{i}\right| \frac{\mu_{T}}{\rho \sigma_{\rho}} \frac{\partial \rho}{\partial x_{i}}
$$




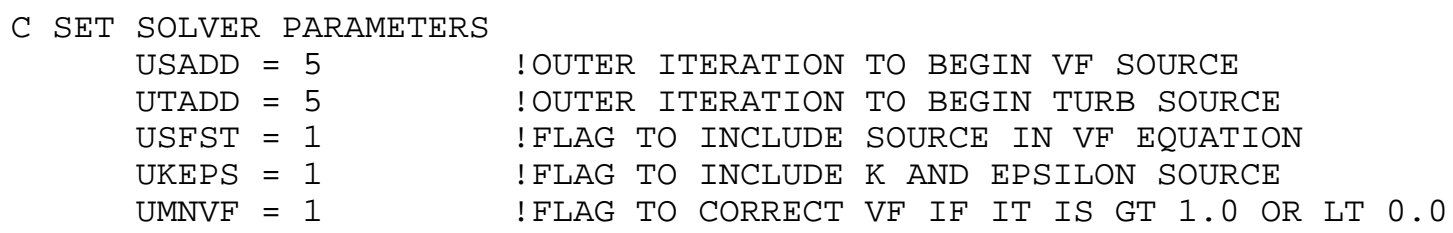

The parameters that are set under SET SOLVER PARAMETERS determine when the source terms are set during the course of the outer iterations and which source terms are included in the simulation. USADD controls on what outer iteration the source term is added to the volume fraction equation. UTADD controls on what outer iteration the source term is added to the $k$ and $\varepsilon$ equations for a simulation that is running the $k-\varepsilon$ model. USFST determines whether the source term will be set in the volume fraction equation; a value of 1.0 turns this option on. UKEPS controls whether the source terms will be set in the $k$ and $\varepsilon$ equations; the value of 1.0 turns this option on. UMNVF turns on an option to set any volume fractions that are less that zero or greater than one to either zero if the volume fraction is less than zero or one if the volume fraction is greater than one. This is done at the end of each outer iteration. If the volume fraction is out of bounds by more than 0.001 the cell location and value of the volume fraction are written to a file that the user can check at the end of the simulation. Like the other flags, a value of 1.0 turns UMNVF on.

The CFX user subroutines USRINT, USRDEN, USRSRC, and USRTRN are included in the file SFST. $f$ and are briefly described. USRINT can be used for setting the initial condition for the simulation. It is called by CFX at the beginning of the simulation; however, if the user desires to initialize the domain from the command file nothing needs to be set in USRINT for the SFST model to run. USRINT is included mainly for the users convenience. USRDEN is used to set the mixture density equation. The user should not need to modify this subroutine. USRSRC is where the source terms for the volume fraction, $k$ and epsilon equations are set. No modifications should be necessary to this subroutine if the user just wants to run the SFST model. USRTRN is included in case the user wants to monitor or write out certain variables during the course of a transient simulation. CFX will call this subroutine, but there are no variables that are necessary for the user to set in this subroutine. UGRDNT and LENGTH are both used by USRSRC. 


\section{B.4 Running a Simulation and Obtaining a Solution}

Several recommendations for running the SFST model are included in this section. These recommendations were observed during many of the simulations that were performed using the SFST model or were determined by numerical experiments.

Since the volume fraction or scalar equation is highly coupled to the density, it is wise to under-relax the lightphase volume fraction equation somewhat more than the momentum equations. This allows the flow field to become established and settle down without huge changes in the density initially.

Since a velocity field that satisfies conservation of mass is very important in not allowing the mean flow to cause the fuel volume fraction to exceed one or become less than zero it is wise to set the residual reduction factor for pressure to a smaller value in comparison to the default value recommended by CFX. The value given under REDUCTION FACTORS by

>>REDUCTION FACTORS

PRESSURE 0.05

has been used successfully. This makes sure that whatever velocity field that is passed to the volume fraction equation, though not necessarily satisfying the momentum equations early in the outer iterations, will still satisfy the continuity equation.

Use first order unwinding for both the density and the volume fraction equation in the command file. The CFX 4.1 Users Manual recommends this for its multiphase models on page 68. As far as the other differencing schemes go, there use is left up to the user. The following have been used with reasonably good success.

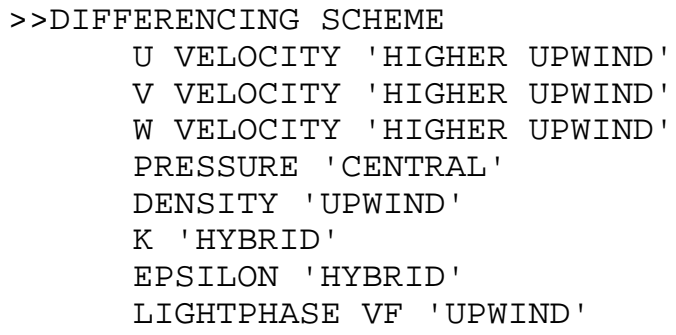

Other than these recommendation, which are closely tied to the SFST model and its equations, it is wise to try deferred correction on the turbulence quantities and decreasing the time step if convergence problems persist. 


\section{B.5 Details of Fortran Implementation of SFST Model in CFX}

This section will focus on the user Fortran in the CFX subroutine USRSRC, where the source terms are set. This subroutine is called before the source is set for each of the variables during the outer iterations. The subroutine USRDEN will also be discussed.

Initially in the code in USRSRC an IF statement determines if any calculations are done or sources are set. The code that determines if source terms are set is given by

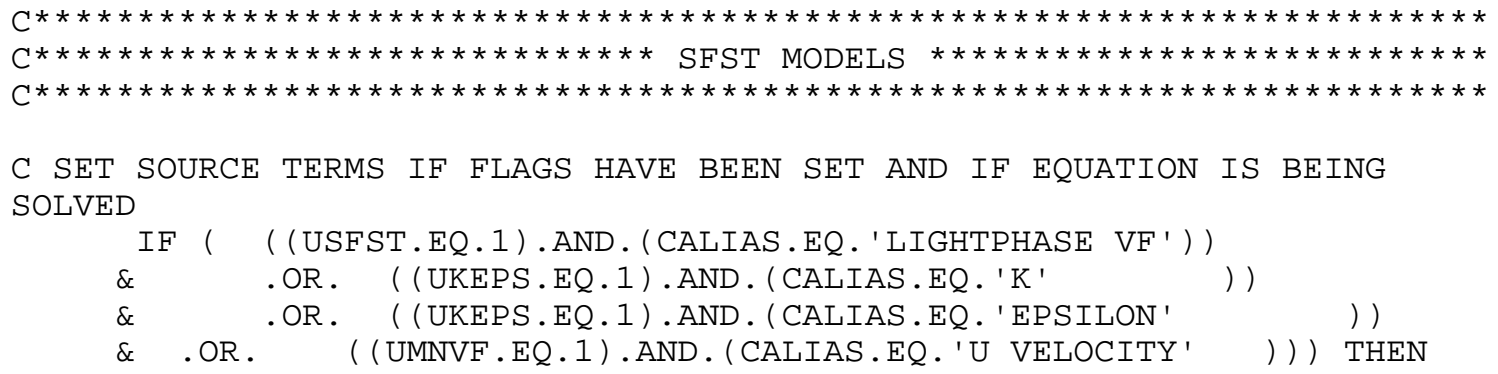

and the decision to set source terms is based on the flags that the user has set in UBCND and which equation CFX is getting ready to solve. After entering the main IF statement the code initializes the character arrays that hold the names of the blocks in which sources are to be set. The initialization code is given by

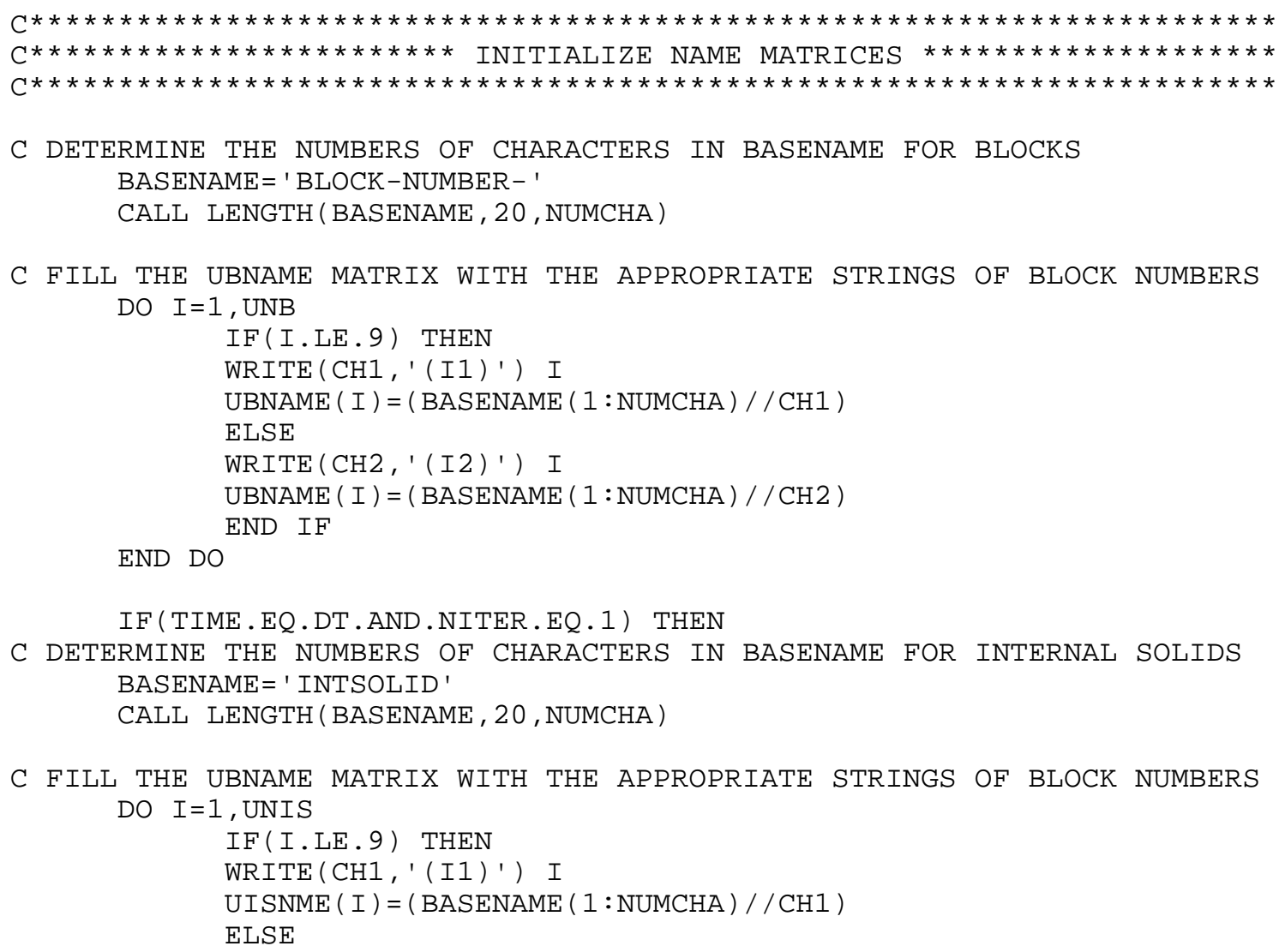




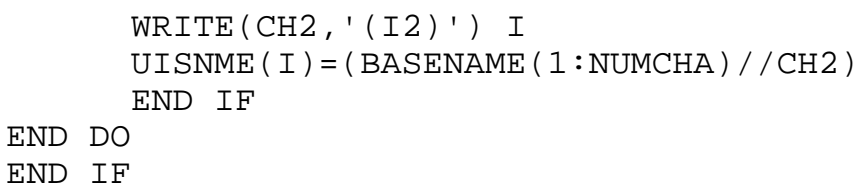

If there are internal solid blocks that are used as child constraints, the subroutine determines which internal node numbers in CFX are used to define the nodes that make up the solid blocks. The values of these internal node numbers are stored in the array UISBLK (UNISC) where UNISC is the total number of nodes that exist in solid blocks that are used as child constraints. The internal numbers for the solid blocks are found in

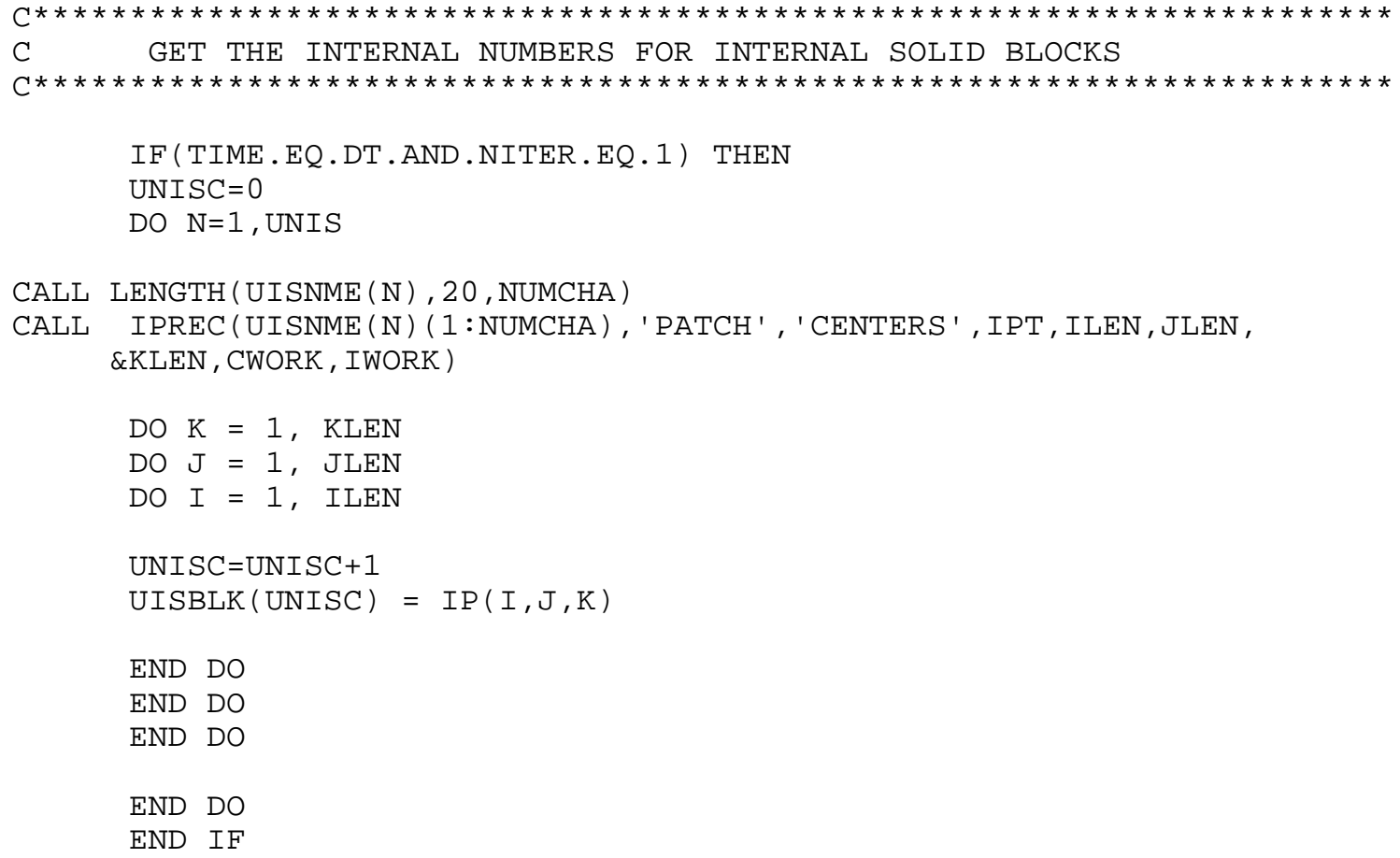

Next the subroutine loops over the entire domain and sets a cell indicator, an integer value, into the user array USRD CELLID, which is referenced in the Fortran by the name SCAL ( INODE , 1, ISCCI). As was mentioned in the section dealing with setting up the geometry file, the SFST model can implement boundary conditions for the slip velocity on five different CFX patches. The boundary conditions for the slip velocity are as follows:

The slip velocity is zero on any wall patch or thin surface patch that is normal to the slip velocity since no fluid can travel through a solid wall. At any inlet, pressure boundary, or mass flow boundary the derivative of the slip velocity normal to the flow is set to zero. This is the typical neumann condition that is set for most quantities at the outlet. 
The cell indicators indicate whether a cell is next to a high or low wall and whether a cell is next to a high or low inlet, pressure boundary, or mass flow boundary. The cell indicators are set according to

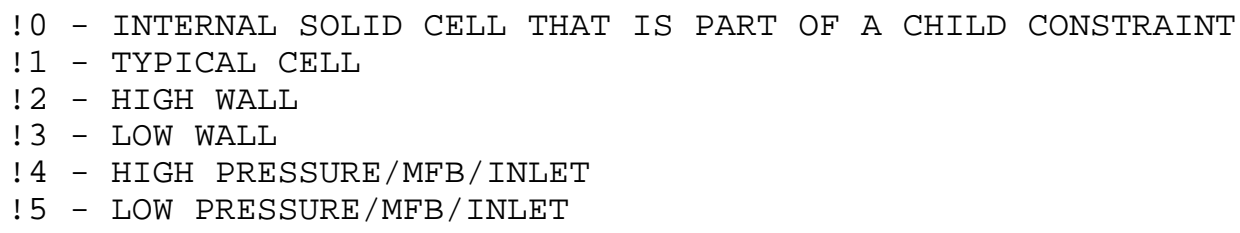

in the following Fortran.

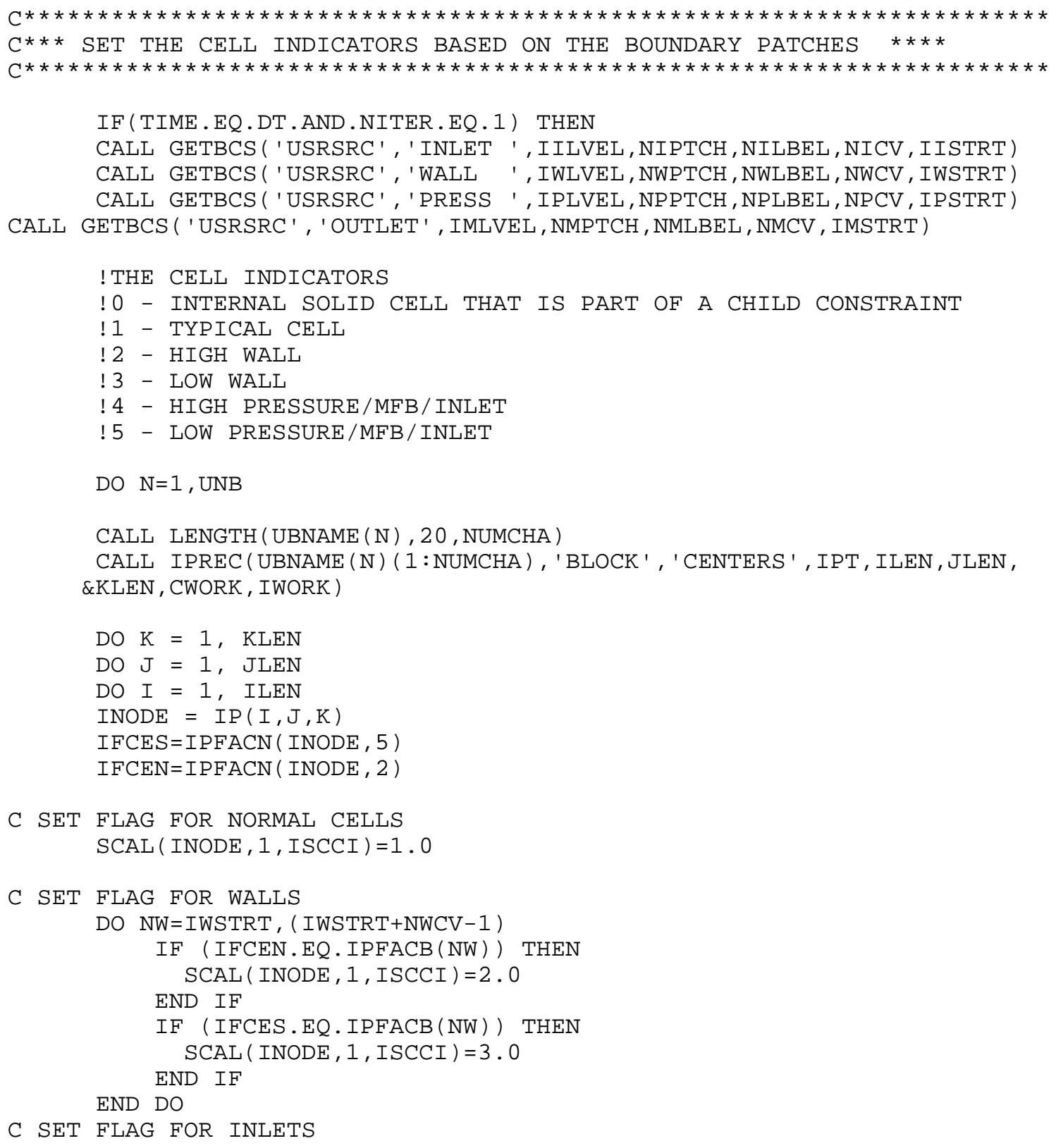




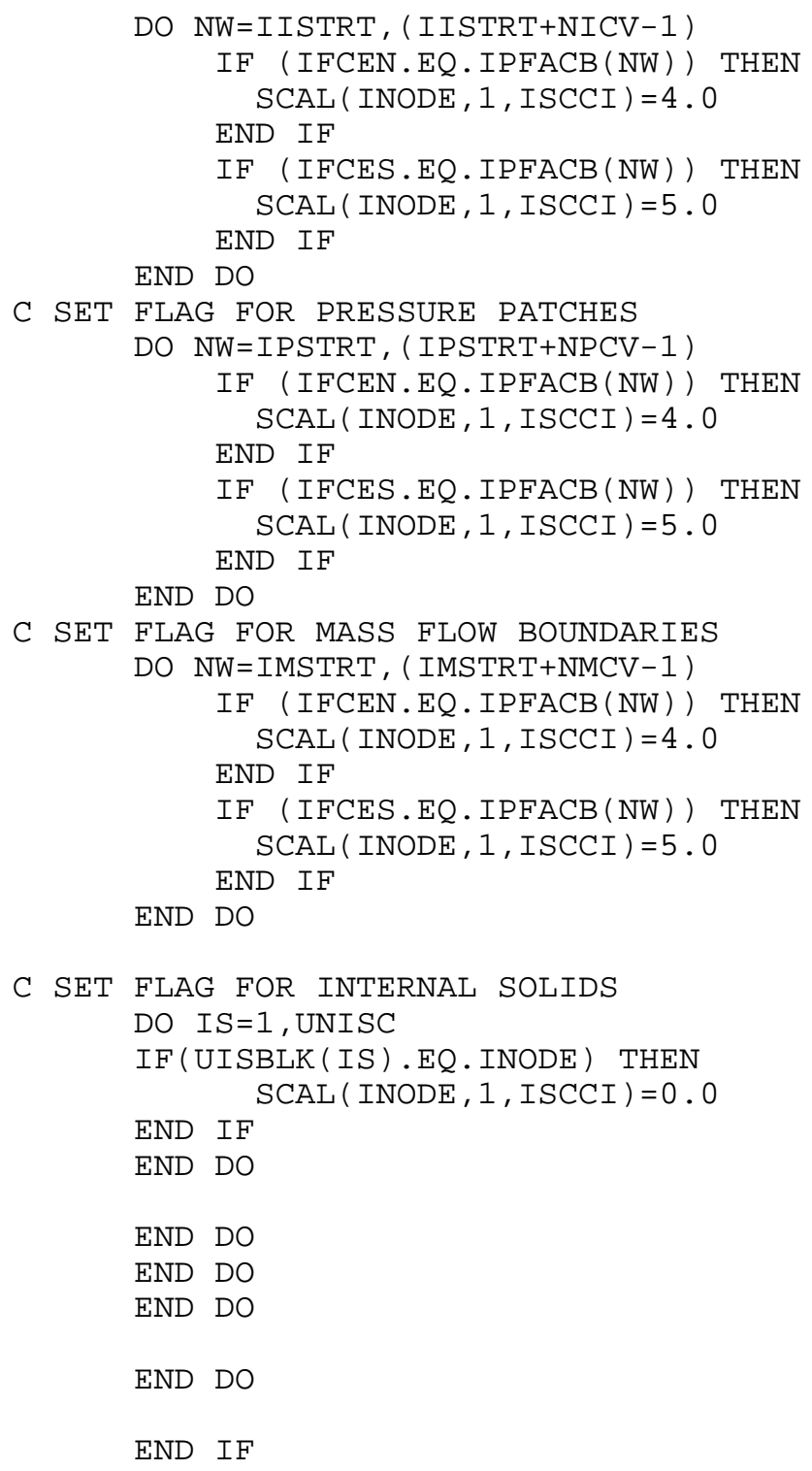

If the user has chosen to monitor and correct slight fluctuations in the boundedness of the volume fraction this is done next in USRSRC as is illustrated below. For any occurrence where the volume fraction is either less than 0.0 or greater than 1.0 the value of the volume fraction before correction, the outer iteration number, the time, the block number, and the vertical location are written to either negvf.txt or posvf.txt. The corrections to the volume fraction are made after the end of an outer iteration, before the next outer iteration begins. 


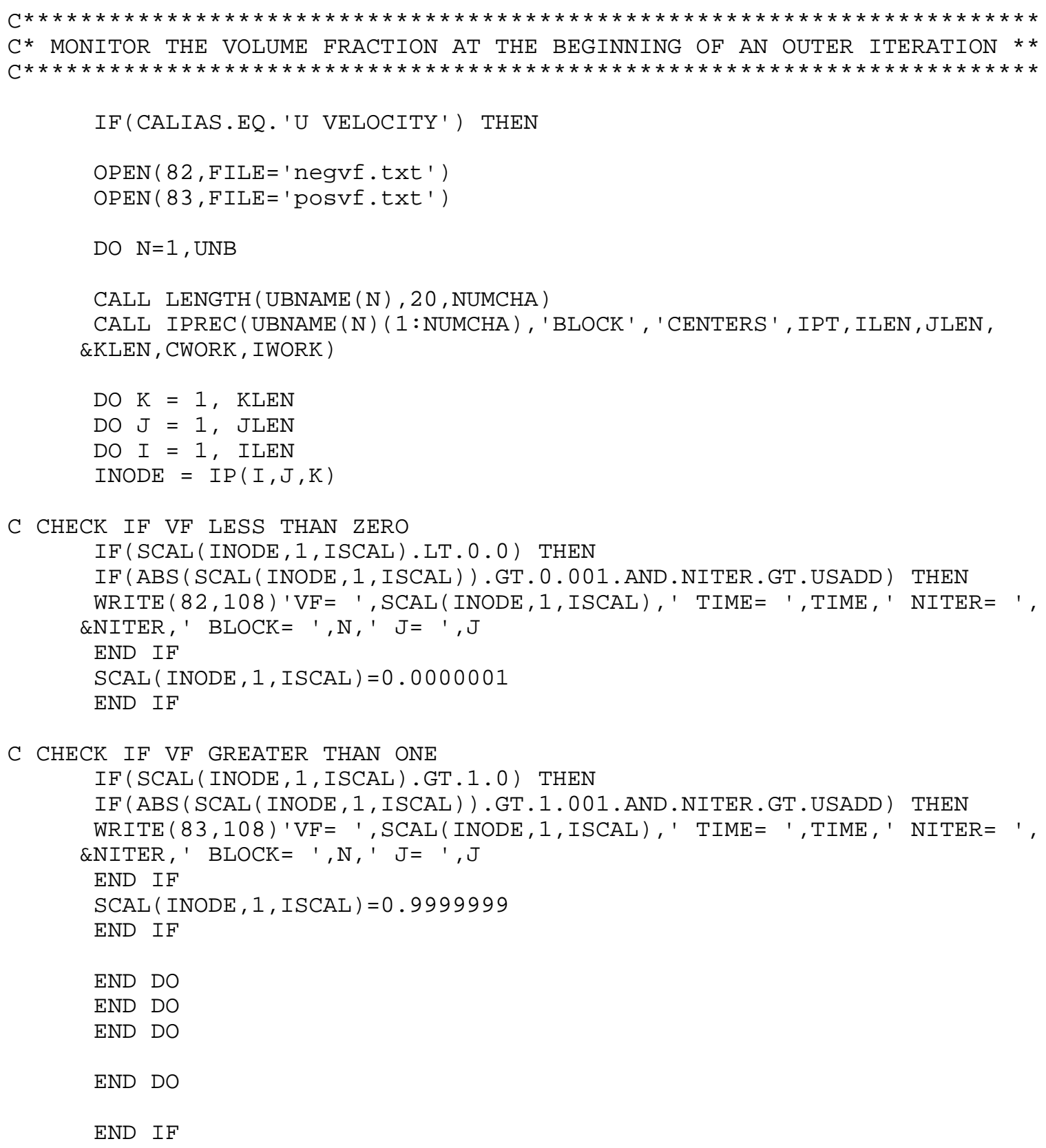

Next the droplet diameter is set in the user array USRD DP, which is referred to in the Fortran as SCAL (INODE, 1, ISCDP). In the present form of the model the droplet size is set to a constant in all the cells; the constant is set in UBCND and the array is filled with the value of the droplet diameter in USRSRC as is demonstrated in the next Fortran segment. If the user was going to implement a dynamic droplet model in the Fortran, the droplet function should be set in the following loop. 


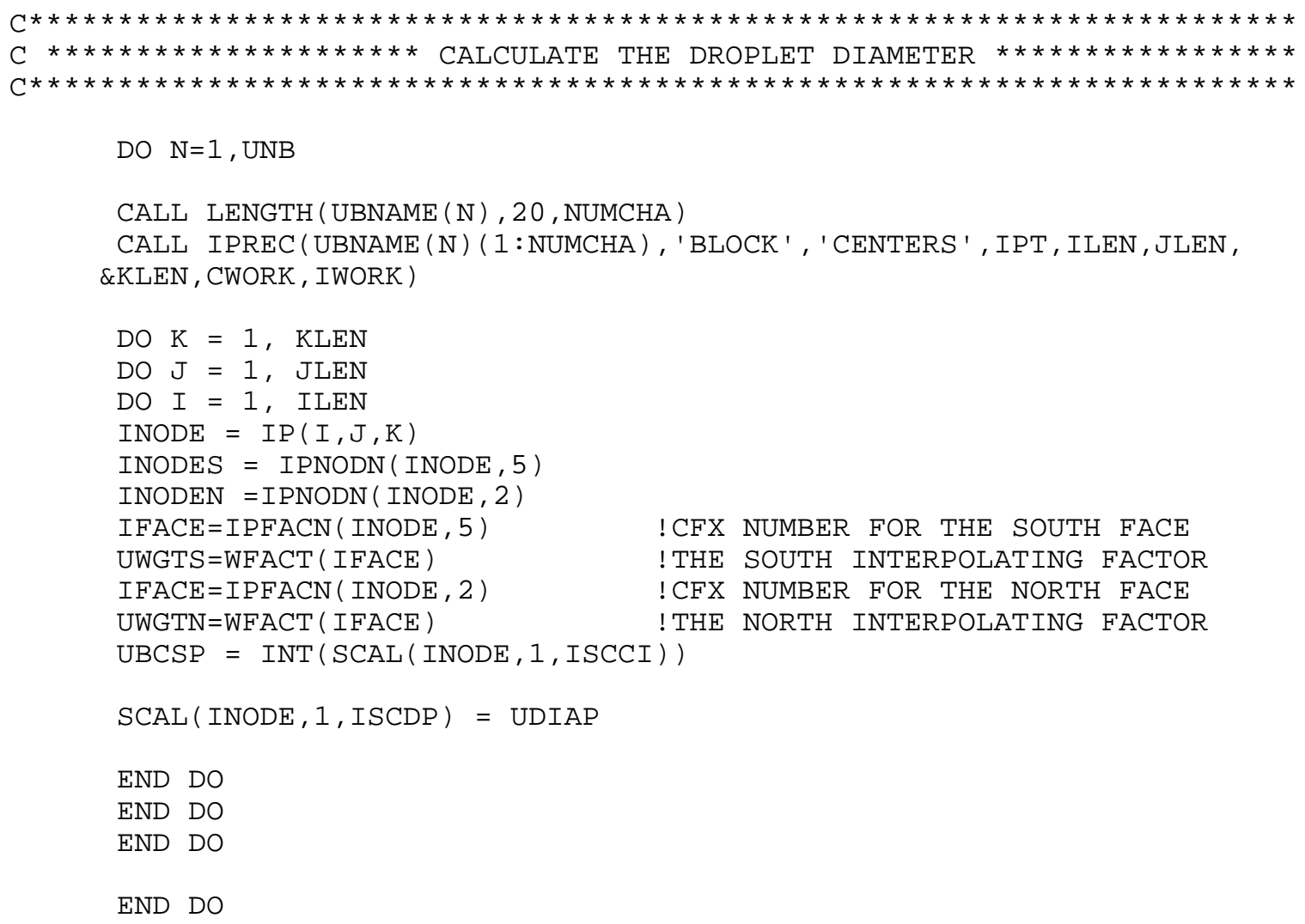

Next the source term involving the slip velocity is set in the volume fraction equation. The slip velocity equation is given by

$$
u_{S}=\left[\left(\frac{4 g d_{p}^{1.6}}{54 \mu_{\beta}^{0.6}}\right)\left(\frac{\rho_{\beta}-\rho_{\alpha}}{\rho_{\beta}^{0.4}}\right)\right]^{5 / 7}\left(1-r_{\alpha}\right)^{\text {UEXPN } 2}\left(r_{\alpha}\right)^{\text {UEXPN1 }}
$$

and the source term that appears in the volume fraction equation is given by

$$
\mathrm{S}=-\rho_{\mathrm{m}} \frac{\partial}{\partial \mathrm{x}_{\mathrm{i}}}\left[\frac{\mathrm{r}_{\alpha} \mathrm{r}_{\beta} \rho_{\beta}}{\rho_{\mathrm{m}}} \mathrm{u}_{\mathrm{S}}\right]
$$

One note should be made here concerning the boundedness of the volume fraction as it relates to the slip velocity. It is found that the formulation given by

$$
\begin{aligned}
& \left.u_{s}\right|_{s}=\operatorname{Min}\left[\operatorname{Max}\left(\left.u_{s}\right|_{s}, 0.0\right), \beta \frac{V_{i}\left(1-r_{i}\right)}{\Delta t_{s_{s}} r_{s}}, \beta \frac{V_{i-1}\left(r_{i-1}\right)}{\Delta A_{s} r_{s}}\right] \\
& \left.u_{s}\right|_{n}=\operatorname{Min}\left[\operatorname{Max}\left(\left.u_{s}\right|_{n}, 0.0\right), \beta \frac{V_{i+1}\left(r_{i+1}\right)}{\Delta t A_{n} r_{n}}, \beta \frac{V_{i}\left(1-r_{i}\right)}{\Delta t A_{n} r_{n}}\right]
\end{aligned}
$$


is very useful in ensuring boundedness by allowing any values of volume fraction above one or less than zero to be corrected during the course of the outer iterations in the solver. The nomenclature as is used in Eq. (5) and Eq. (6) is as follows: V is the cell volume, A is the area normal to the slip velocity, $\mathrm{r}$ is the volume fraction of the light phase, $\Delta \mathrm{t}$ is the time step used in the discretisation, and $\beta$ is the boundedness parameter that the user sets as USBND in UBCND. Subscripts $s$ and $n$ refer to the south and north faces on a control cell and the subscript $\mathrm{i}$ refers to the cell in question, with $\mathrm{i}+1$ indicating a cell located above cell i and i-1 indicating a cell below cell i . It can be seen by comparing Eq. (5) and Eq. (6) that the correction provided by these equations is conservative in that it can only spatially redistribute the volume fraction, not destroy or produce it.

Another issue that may be of some concern to the user involves how the flux due to the slip velocity is calculated in the vertical direction when the grids are non-orthogonal. The solution to this problem is facilitated by the fact that CFX stores the three area compents on each of the six faces. Hence when the flux due to the slip velocity is calculated, in the vertical direction, only the area component normal to the vertical direction is used.

It should also be mentioned that if the user wants to set different relationships for the slip velocity it should be done in the following loop where the source terms are set in the volume fraction equation.

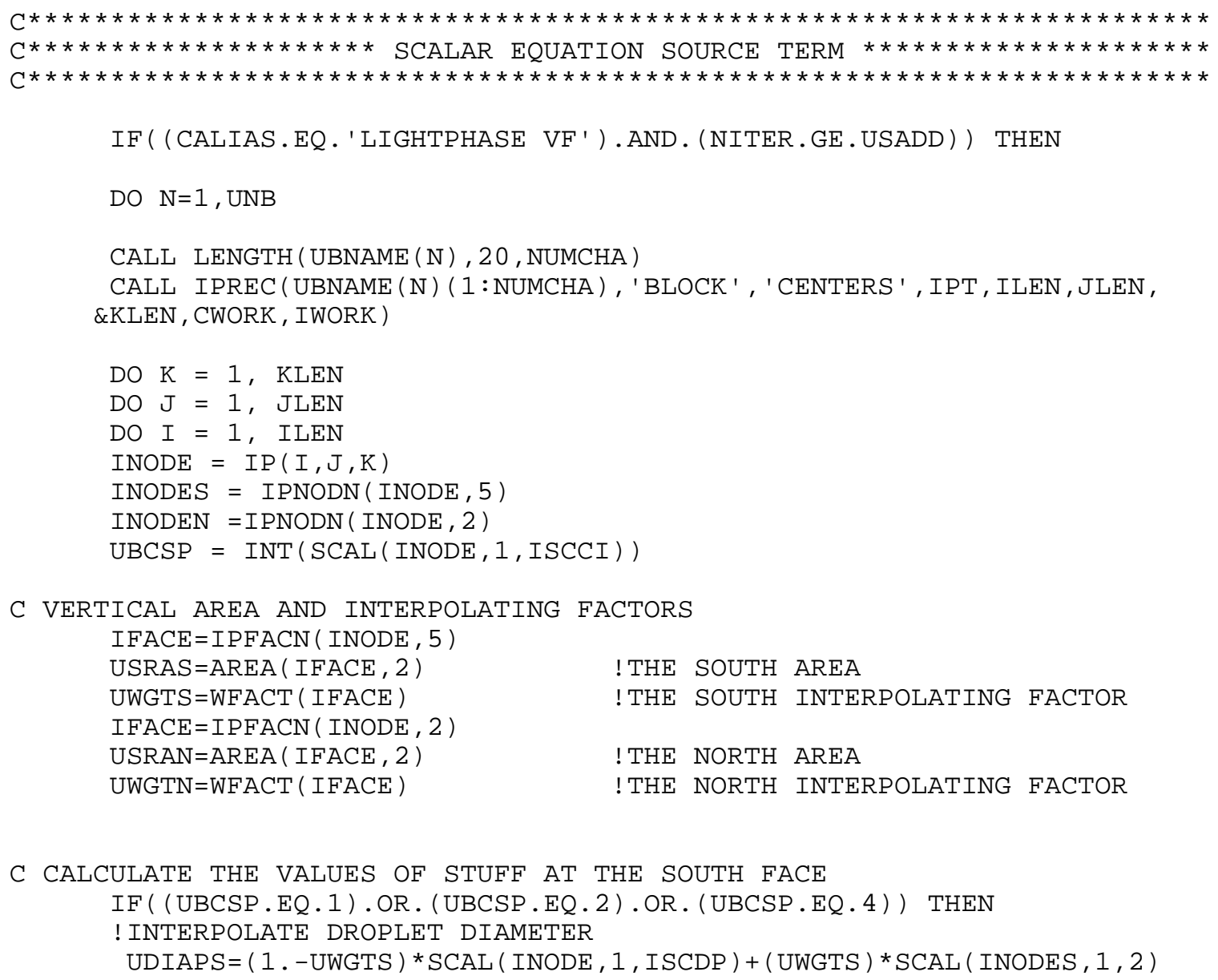




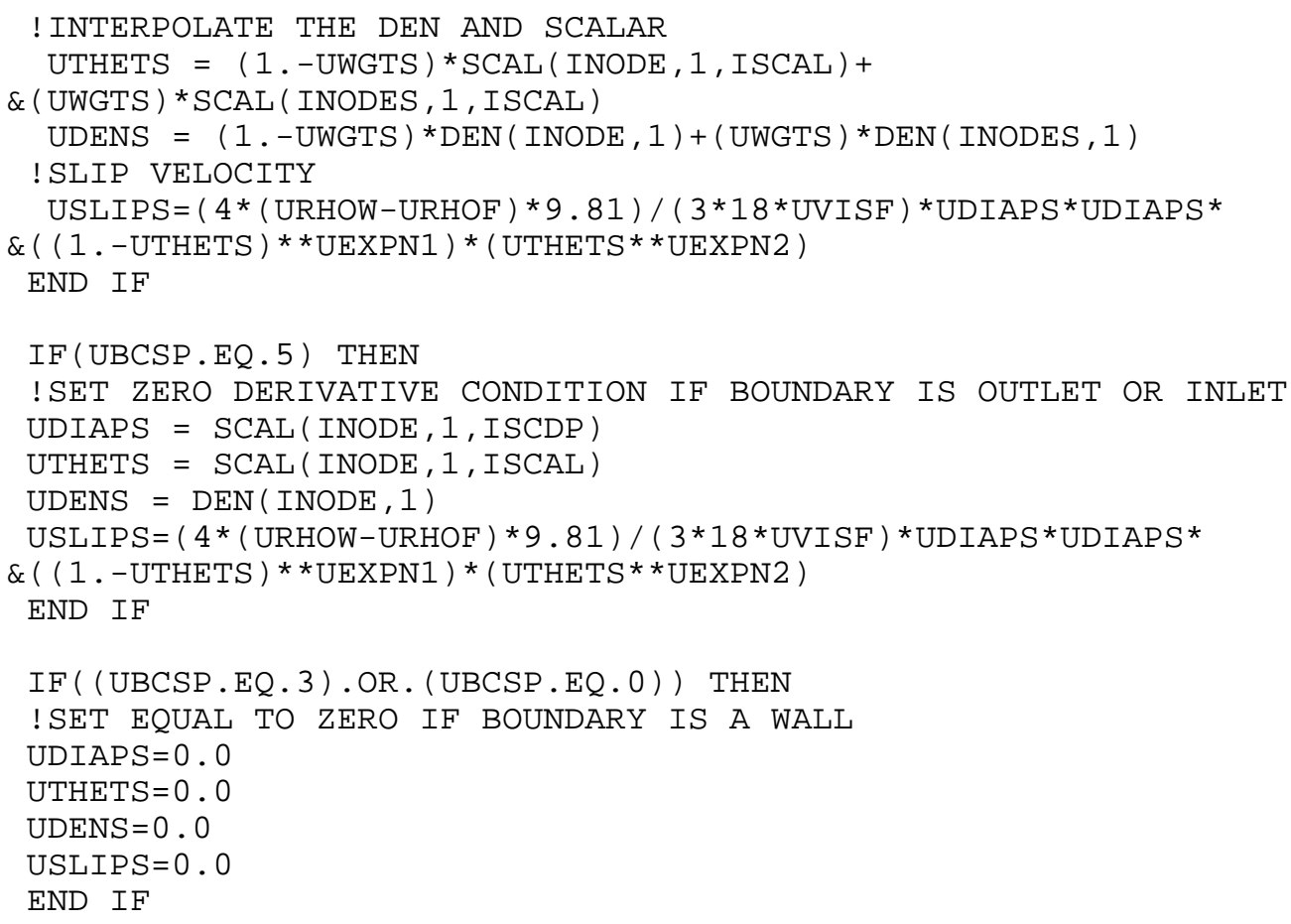

C LIMIT THE SCALAR SOURCE TO GIVE BOUNDED VOLUME FRACTIONS

C SOUTH SOURCE IF ( (UBCSP.EQ.1) .OR. (UBCSP.EQ.2) .OR. (UBCSP.EQ.4)) THEN USLS $1=U T H E T S *$ USRAS *USLIPS * (1 . -UTHETS) *URHOW/MAX (UDENS, URHOF) $\operatorname{USLS} 2=\mathrm{USBND} *(\operatorname{VOL}(\mathrm{INODE}) *(1 .-\operatorname{SCAL}(\mathrm{INODE}, 1, \mathrm{ISCAL}))) /(\mathrm{DT})$ 


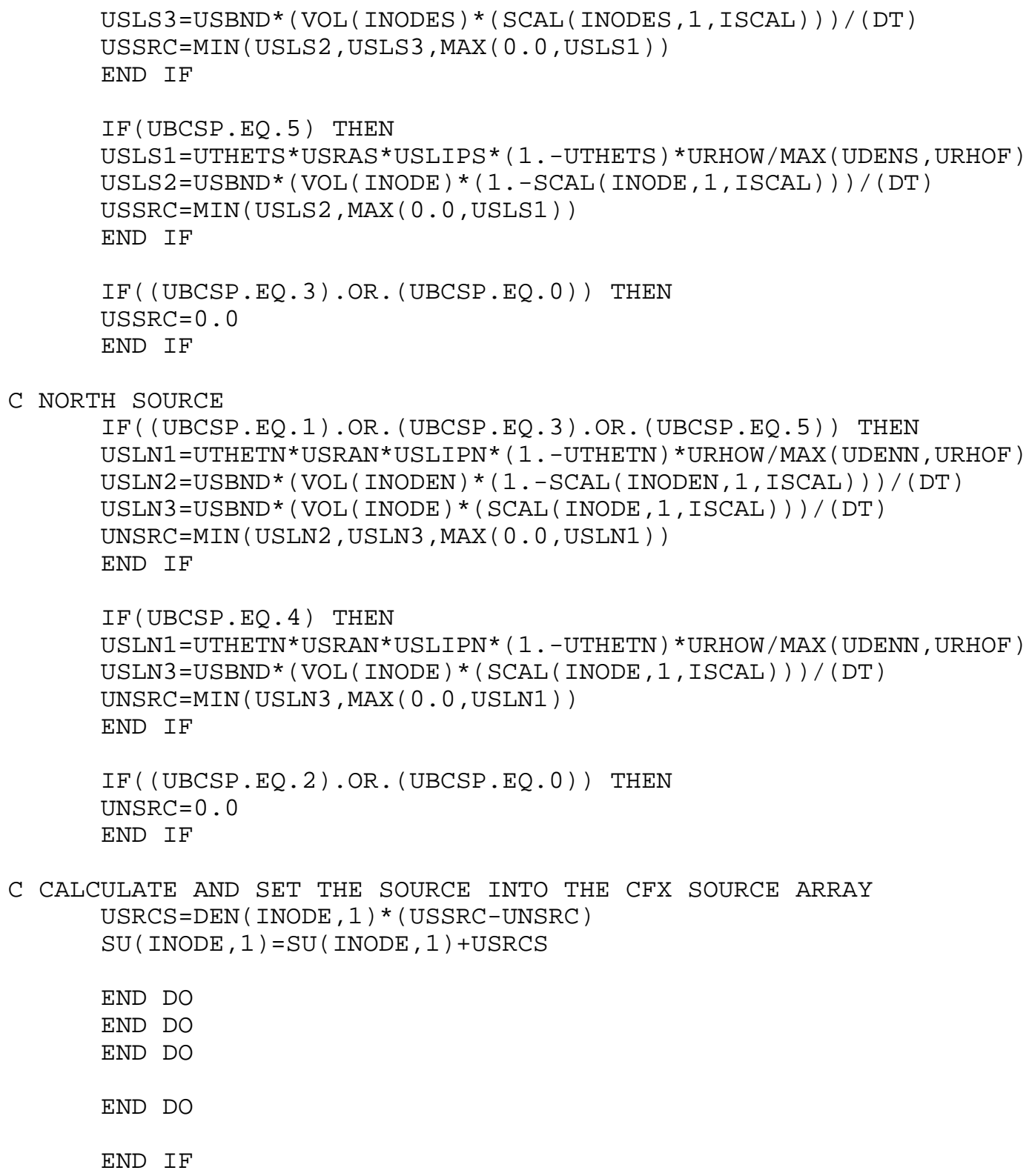

The source terms for the $k$ and $\varepsilon$ equations are set next in the Fortran in USRSRC. Since these source terms require the density derivative in the vertical direction. The gradients are calculated using the subroutine UGRDNT. This subroutine will calculate the gradient of any quantity as follows:

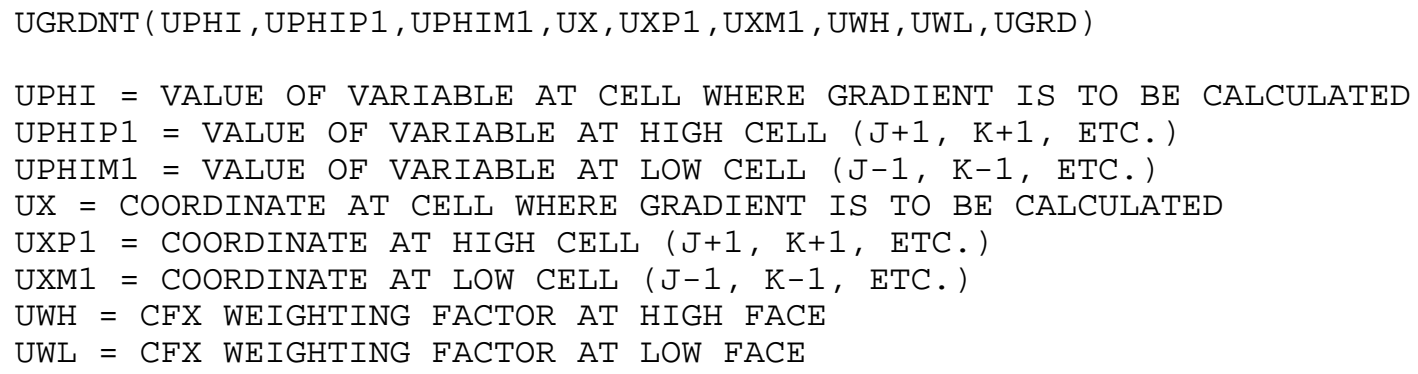


The gradient is calculated by interpolating for values of the variable and the coordinates at the high and low faces defining the cell where the gradient is to be calculated. This is done using linear interpolation as:

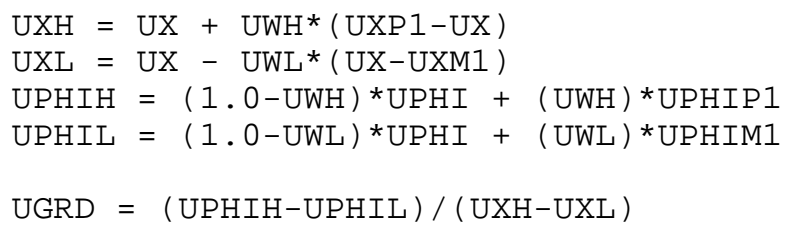

For cells adjacent to boundaries the subroutine can still be used, it is just necessary to use a one sided difference by setting

$\begin{array}{ll}\text { UPHI }=\text { UPHIP } 1 & \text { for a cell on a high boundary } \\ \text { UPHI=UPHIM1 } & \text { for a cell on a low boundary }\end{array}$

when the subroutine is called. The source term for the destruction of $k$ is also limited in that the Fortran will not allow the code to destroy more $k$ than physically resides in a cell and the source term is set to zero if $k$ is zero.

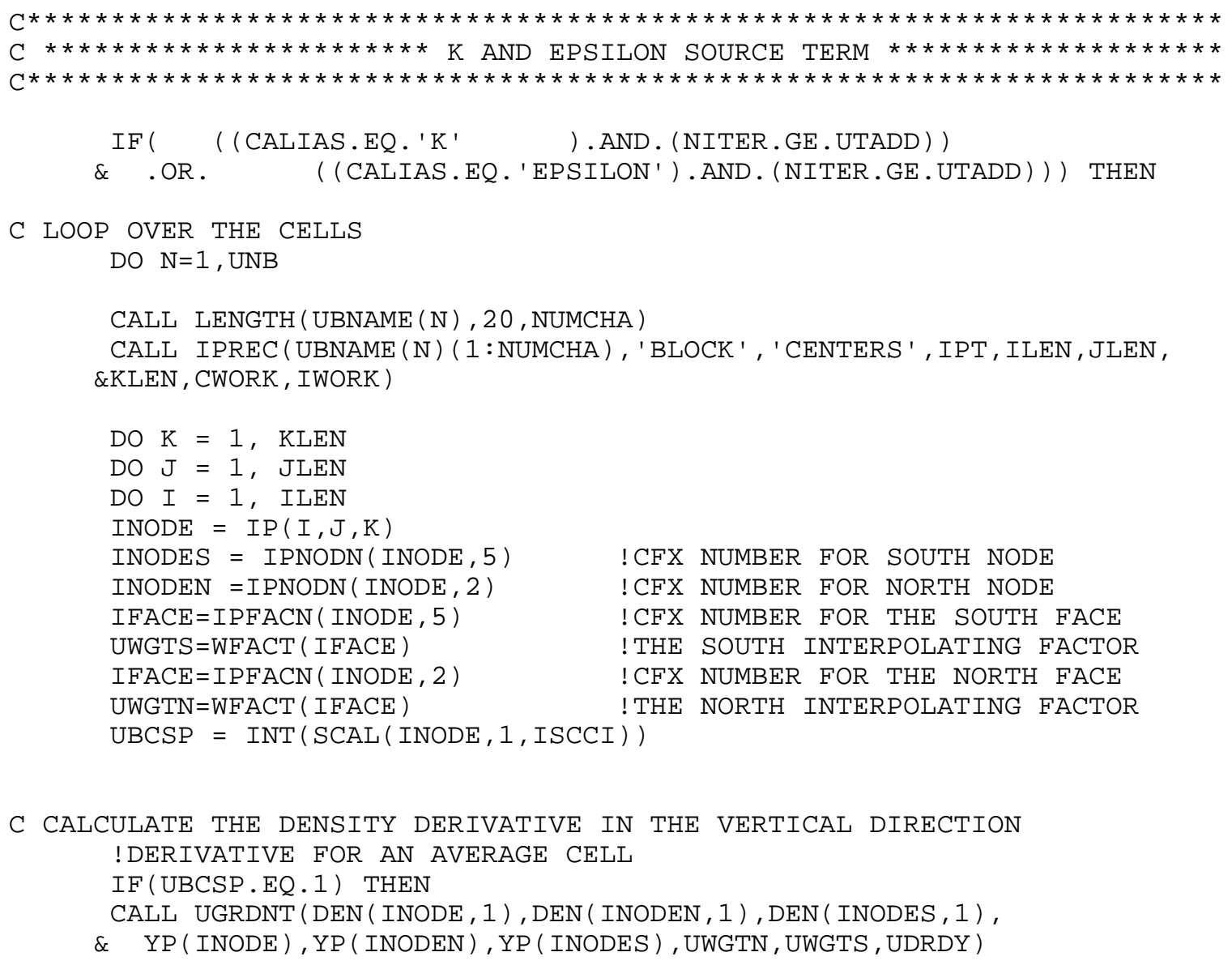




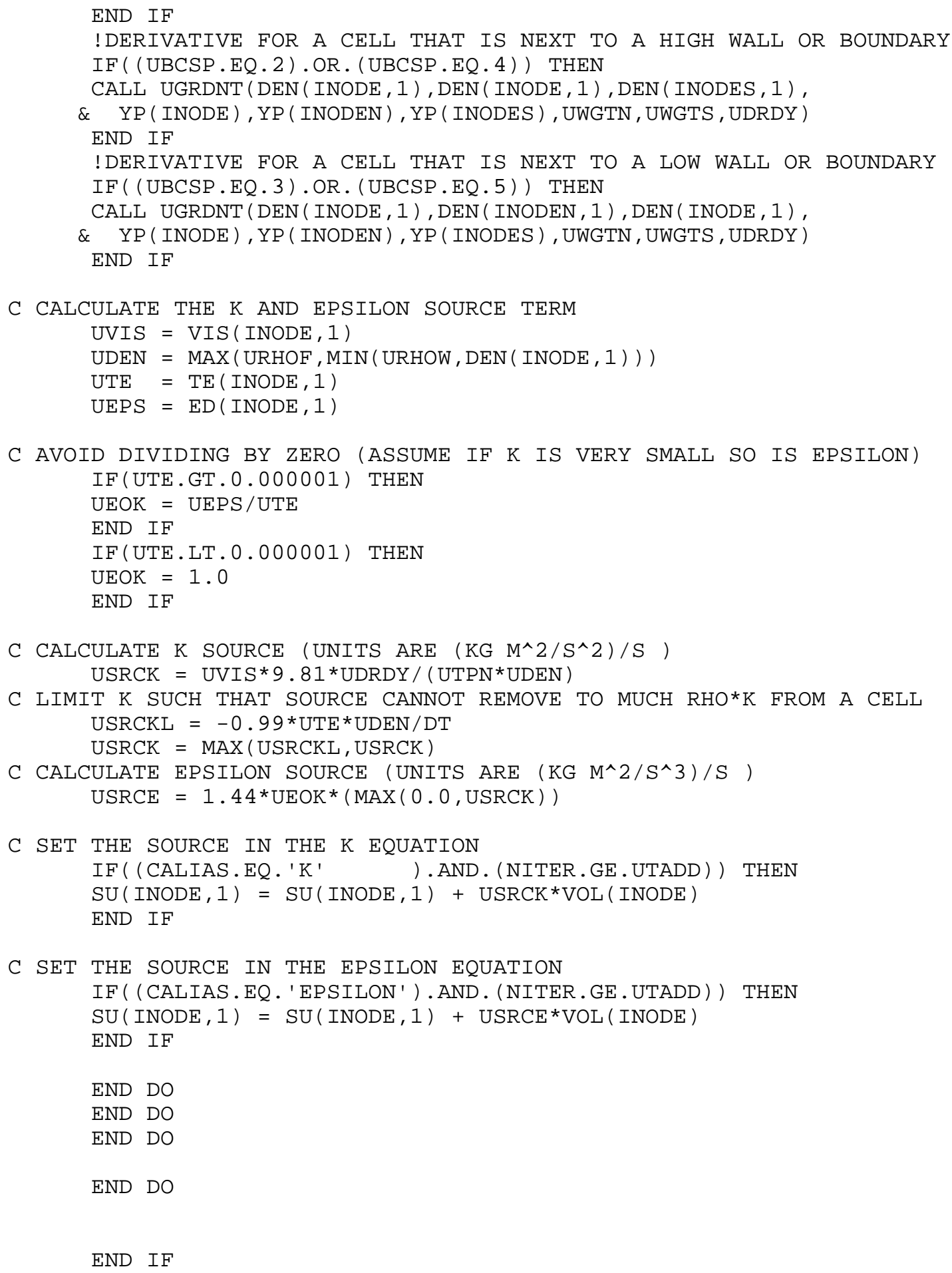

The mixture density equation is set in the CFX user subroutine, USRDEN. The following code in USRDEN is used to implement the mixture density equation as a function of the un-mixed phase densities and the local volume fraction. 


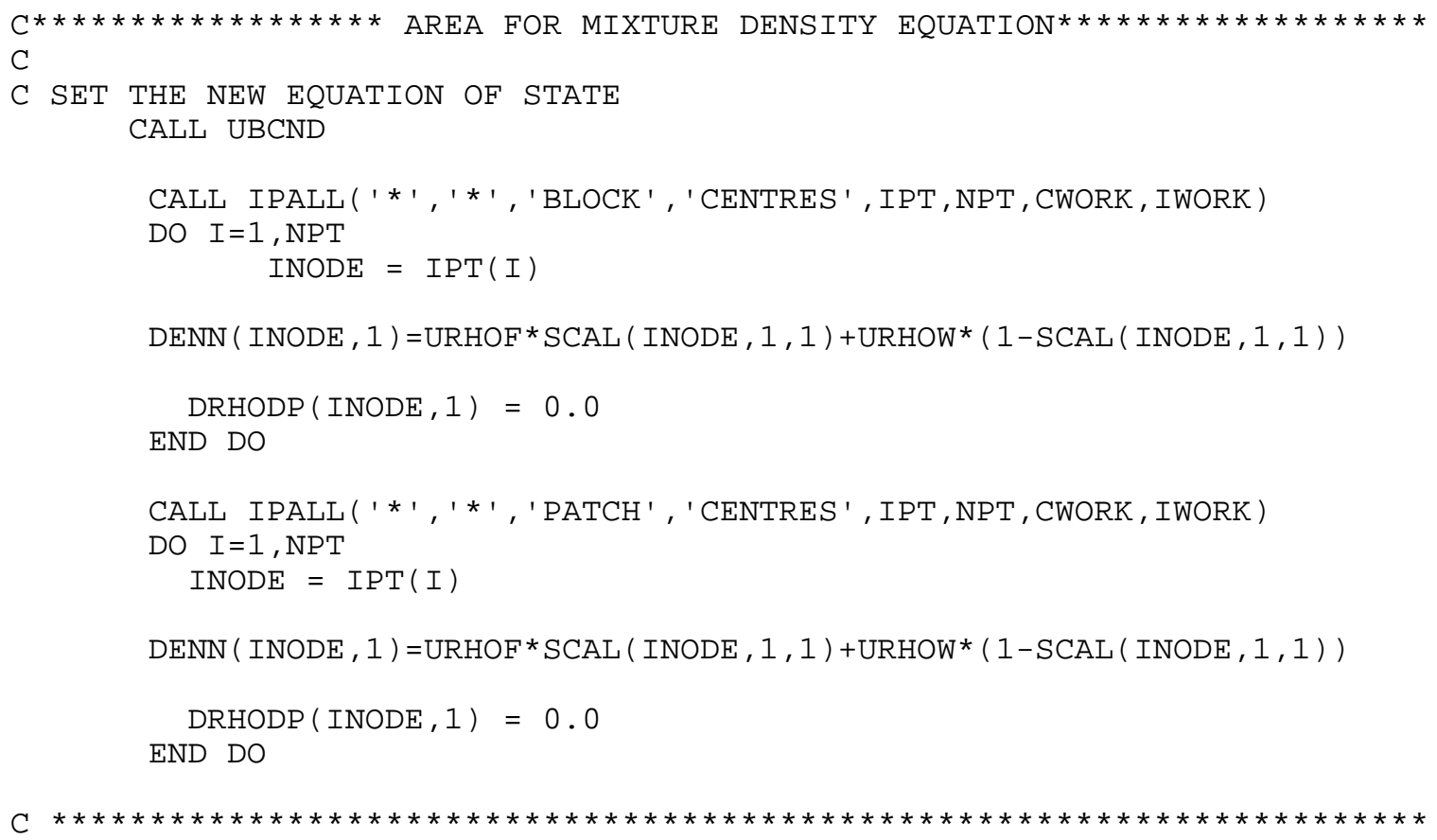

More extensive details on the different arrays and utility routines that are unique to CFX 4.1 can be found in the CFX Users Manuel. Many of these routines are used quite frequently in the Fortran that implements the SFST model. 


\title{
PREDICTION OF TURBULENT MIXING AT THE INTERFACE OF DENSITY STRATIFIED, SHEAR FLOWS USING CFD
}

By

\author{
Matthew R. Umbel
}

\author{
A THESIS \\ Submitted to \\ The College of Engineering and Mineral Resources \\ at \\ West Virginia University \\ in partial fulfillment of the requirements \\ for the degree of \\ Master of Science \\ in \\ Mechanical Engineering
}

APPROVAL OF EXAMINING COMMITTEE

John Kuhlman, Ph.D.

Rakesh Gupta, Ph.D.

Date

Ismail Celik, Ph.D., Advisor 